\begin{abstract}
Title of Document:

Directed By:

COMPLEX SELF-SORTING SYSTEMS

Soumyadip Ghosh, Ph.D., 2009

Professor Lyle D. Isaacs

Department of Chemistry and Biochemistry
\end{abstract}

Over the past century scientists have taken a reductionist approach towards much of the physical and biological sciences. More recently scientists have become interested in constructing complex systems from their components and thereby controlling their emerging behaviors. As supramolcular chemists we have been pursuing an approach to the creation of complex functional systems - systems chemistry - by preparation of self-sorting systems. Self-sorting system displays ability to efficiently distinguish between self- and non-self even within complex mixture. This dissertation is divided into four chapters that describe increasingly complex self-sorting systems.

Chapter 1 describes a literature review on self-sorting. First, we introduced the concept of self-sorting and then described the previously studied self-sorting phenomenon in the Isaacs group followed by a description of the examples of selfsorting systems in the literature. 
Chapter 2 describes the synthesis and characterization of eleven C-shaped methylene bridged glycoluril dimers (II-1 - II-11) bearing H-bonding groups on their aromatic rings. Compounds II-1, II-2, $( \pm)-\mathbf{I I}-\mathbf{4 a},( \pm)-\mathbf{I I}-5$, and II-7 form tightly associated homodimers in $\mathrm{CDCl}_{3}$ due to $\pi-\pi$ and $\mathrm{H}$-bonding interactions. Compounds II-2, ( \pm )-II-5 and II-7, having disparate spatial distribution of their Hbonding groups show the ability to efficiently distinguish between self and non-self within three component mixtures in $\mathrm{CDCl}_{3}$. The effect of various structural modifications (e.g. chirality, side chain steric bulk, relative orientation, number and pattern of H-bonds) on the strength of self-assembly and the fidelity of self-sorting are presented.

Chapter 3 describes the stepwise construction of an 8-component self-sorted system (III-1 - III-8) by the sequential addition of components. This process occurs via a large number of states $\left(2^{8}=256\right)$ and even a larger number of pathways $(8 !=$ 40320). A pathway (III-5, III-6, III-7, III-8, III-4, III-3, III-2, then III-1) that is self-sorted at every step along the way. Another pathway (III-1, III-8, III-3, III-5, III-4, III-7, III-2, then III-6) exhibits interesting shuttling of guest molecules among hosts. The majority of pathways - unlike the special ones described above - proceed through several non self-sorted states. We characterized the remainder of the 40320 pathways by simulation using GEPASI and describe the influence of concentration, mean binding constants and standard deviation on the fidelity of the self-sorting pathways.

Chapter 4 describes a method to control biological catalysis using synthetic self-sorting systems. We report the synthesis of IV-1 - IV-5 which contain both 
enzyme inhibitor and cucurbit[n]uril binding domains. The enzyme binding domains of IV-1 - IV-5 bind to the active sites of Bovine Carbonic Anhydrase or Acetylcholinesterase and inhibit their catalytic activities. Addition of $\mathrm{CB}[7]$ catalyzes the dissociation of IV-1 and IV-2 from the active site of BCA and thereby regenerates the enzymatic activity. In contrast, addition of $\mathrm{CB}[7]$ to $\mathrm{AChE} \cdot \mathbf{I V}-\mathbf{4}_{4}$ and $\mathrm{AChE} \cdot \mathbf{I V}-\mathbf{5}_{4}$ results in the formation of a ternary complex that does not regenerate the enzymatic activity. 


\title{
COMPLEX SELF-SORTING SYSTEMS
}

\author{
By
}

Soumyadip Ghosh

Dissertation submitted to the Faculty of the Graduate School of the University of Maryland, College Park, in partial fulfillment of the requirements for the degree of

Doctor of Philosophy 2009

Advisory Committee:

Professor Lyle Isaacs, Chair

Professor Jeffery Davis

Assistant Professor Andrei Vedernikov

Professor Dorothy Beckett

Associate Professor John Fisher, Dean's Representative 
(C) Copyright by Soumyadip Ghosh 2009 


\section{Dedication}

To my parents, Bipul Kanti Ghosh and Kalpana Ghosh and my wife, Sangita. 


\section{Acknowledgements}

I would like to express sincere gratitude to my Ph.D. advisor Professor Lyle Isaacs for his guidance throughout my graduate studies. I am truly grateful to Lyle for his time, support, motivation, patience and I must say that I have learned a lot from him over the years.

I also have to thank Dr. Jason Lagona for teaching me all the essential skills to become a synthetic organic chemist. I would like to appreciate Dr. Kousik Kundu, Dr. Sriparna Chakraborty and Dr. Pritam Mukhopadhyay for their role as caring seniors. Their advices helped me to get over many difficult situations in graduate school.

It was my pleasure to work with a group of very supportive colleagues in the Isaacs lab. Thank you all. Of all the colleagues and friends I would like to sincerely thank Regan and Becky for their help. I would like to thank Dr. Yiu-Fai Lam and Dr. Yinde Wang for their help with various NMR experiments.

A very special thanks to my past and present roommate Kousik and Debojyoti for all they have done for me.

Finally, this accomplishment would have never been possible without the inspiration and encouragement of my parents and wife. I would like to thank my wife for her support, enduring patience, understanding and putting up with me during all these years. 


\section{Table of Contents}

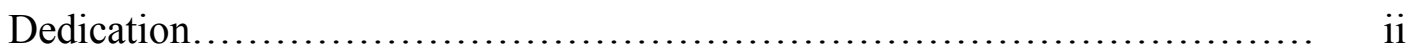

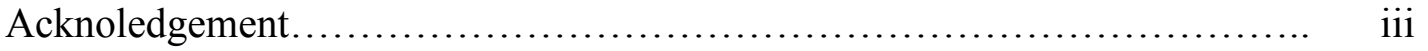

Table of Contents...................................................... iv

List of Tables.......................................................... vii

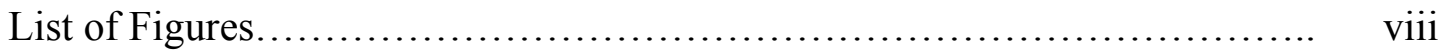

List of Schemes.....................................................

List of Abbreviations................................................ XXv

I. Chapter 1: Complex Self-Sorting Systems

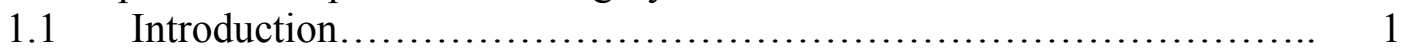

1.2. Self-Sorting .................................................. 2

1.2.1 Early Examples of Self-Sorting........................... 2

1.2.2 Difference Between Self-Assembly and Self-Sorting........... 5

1.3 Self-Sorting Behavior of Molecular Clips............................ 7

1.4 Development of Complex Self-Sorting Systems........................ 9

1.4.1 Self-Sorting: The Exception or the Rule........................ 9

1.5 Examples of Self-Sorting Systems in Aqueous Solution................. 12

1.5.1 Social Self-Sorting in Aqueous Solution....................... 12

1.5.2 The CB[n] Family of Macrocycles are Prime Components for the Construction of Self-Sorting Systems........................ 14

1.5.3 Kinetic Self-Sorting...................................... 17

1.5.4 Self-Sorting Processes Control the Folding, Forced Unfolding, and Refolding of an Abiotic Oligomer in Water...................... 19

1.6 Selected Examples of Self-Sorting Systems from Literature............. 22

1.6.1 Self-sorting Systems Based on Porphyrin Coordination Chemistry 22

1.6.2 Self-sorting Based on Hydrogen Bonding Interactions............ 25

1.6.3 Self-Sorting Coiled Coil.................................... 27

1.6.4 Construction, Substitution, and Sorting of Metallo-organic Structures via Subcomponent Self-Assembly.................. 29

1.7 Application of Self-Sorting in Material Science and Nanotechnology..... 31

1.7.1 Self-Assembly of Polymers and Nanoparticles on Patterned Surfaces.................................................. 31

1.7.2 Geometric Self-Sorting in DNA Self-Assembly............... 32

1.7.3 Self-Sorting in Polymers................................ 34

1.7.4 Self-Sorting Gels....................................... 35

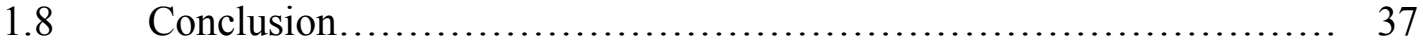

II. Chapter 2: Self-Sorting Molecular Clips.

$2.1 \quad$ Introduction................................................... 40

2.2 Design and Synthesis of Molecular Clips.......................... 42

2.2.1 Design of the Chemical Components Used in this Study......... 42

2.2.2 Synthesis of the Molecular Clips Bearing H-bonding Groups on 
Their Aromatic Ring ....................................... 45

2.3 Self-assembly of Molecular Clips................................. 47

2.3.1 Molecular Clips Form Homodimers in $\mathrm{CDCl}_{3} \ldots \ldots \ldots \ldots \ldots \ldots \ldots . . \ldots 7$

2.3.2 Concept of Geometrical Match and Mismatch.................. 50

2.3.3 X-ray Crystal Structures of II-1, II-2, $( \pm)$-II-4b and ( \pm )-II-5 ..... 53

2.4 Three- and Four- Component Self-Sorting Systems of Molecular Clips.. $\quad 54$

2.4.1 Design Aspects of New Molecular Clips......................... 54

2.4.2 Self-Assembly of II-7 in $\mathrm{CDCl}_{3}$ solution and solid state.......... 55

2.4.3 Three Component Self-Sorting Systems........................ 57

2.4.4 Selective Dissociation of One Member of a Self-Sorted Mixture. 59

2.4.5 Steric Effects Influence the Thermodynamics and Kinetics of Dimerization............................................. 61

2.4.6 The Effect of Antiparallel Orientation of H-bonding Sidearms on Dimerization.............................................. 65

2.4.7 Effect of Additional H-bonding Groups on the Ability of Molecular Clips to Undergo Self-Assembly and Self-Sorting..... 67

2.4.8 Four-Component Self-Sorting Systems....................... 71

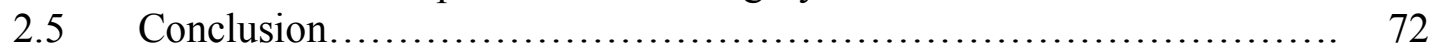

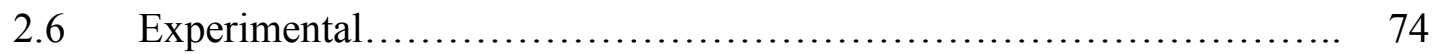

2.6.1 General Experimental.................................. 74

2.6.2 Synthetic Procedures and Characterization..................... 75

2.6.3 NMR Experiments....................................... 88

III. Chapter 3: Deconvolution of a Multi-Component Interaction Network Using Systems Chemistry.

$3.1 \quad$ Introduction.................................................... 90

3.2 Preparation of an 8-Component Self-Sorted Mixture.................... 93

3.2.1 Selection of the Chemical Components Used in this Study 93

3.2.2 Self-Sorting System Comprising III-1 - III-8 ................. 94

3.3 Stepwise Construction of 8-Component Self-Sorted Mixture.............. 95

3.3.1 Molecular Musical Chairs.................................... 96

3.3.2 Statistical Considerations.................................... 98

3.3.3 Computational Approach Towards a Global Understanding of the Stepwise Construction of a Four Component System............. 99

3.3.4 Experimental Investigation of Other Pathways that Lead to the Eight Component Self-Sorted System......................... 102

3.3.5 Computational Approach Towards Global Understanding of the Experimental System Comprising III-1 - III-8.................. 104

3.3.6 Effect of Number of Components on Self-Sorted States........... 107

3.3.7 Effect of Concentration of Components on Self-Sorted States and Pathways................................................ 108

3.3.8 Effect of Sequence of Addition of Components on Pathways...... 111

3.4 The Effect of Equilibrium Constants................................ 113

3.5 Conclusion........................................................ 115 
IV Chapter 4: Biological Catalysis Controlled by a Synthetic Self-Sorting System

4.1 Introduction.................................................... 118

4.1.1 Design Aspects of the Chemical Components Used in this Study 120

4.2 Results and Discussion....................................... 121

4.2.1 Synthesis of Guests IV-1 - IV-5 ..................... 122

4.2.2 Chemical Reactions Used to Monitor Enzymatic Activities........ 123

4.2.3 Regulation of Biological Catalysis of Bovine Carbonic Anhydrase. 125

4.2.4 Measurement of Association Constant............................. 129

4.2.5 Measurement of the $\mathrm{K}_{\mathrm{a}}$ of the Inhibitor to $\mathrm{CB}[7] \ldots \ldots \ldots \ldots \ldots . . . \ldots 129$

4.2.6 Evidence of Shuttling from Fluorescence Spectroscopy........... 130

4.2.7 Measurement of the Association Constant $\left(\mathrm{K}_{\mathrm{a}}\right)$ of the Inhibitor to the BCA..................................................... 131

4.2.8 Kinetic Effect in Enzyme Inhibitor Binding..................... 133

4.2.9 Determination of the Values of $\mathrm{k}_{\mathrm{on}}$ and $\mathrm{k}_{\mathrm{off}}$ of the inhibitor from BCA................................................... 134

4.2.10 Formation of Ternary Complexes BCA • IV-1•CB[7] and BCA • IV-2•CB[7] .......................................... 137

4.2.11 Is the Shuttling of the Two-Faced Guest a Common Behavior?.... 138

4.2.12 Regulation of Biological Catalysis of Acetylcholinesterase........ 139

4.2.13 Measurement of Association Constants........................ 139

4.2.14 Fluorophore Displacement Assay............................. 142

4.2.15 Effects of CB[7] in Controlling Enzymatic Activity of AChE..... 143

4.2.16 The Origin of the Difference in the Behavior of Two Enzymes.... 144

4.3 Conclusion..................................................... 146

$4.4 \quad$ Experimental...................................................... 147

4.4.1 General Experimental..................................... 147

4.4.2 Synthetic Procedures and Characterization...................... 148

4.4.3 ${ }^{1} \mathrm{H}$ NMR Competition Experiments.......................... 152

4.4.3.1 Sample Determination of $\mathrm{K}_{\mathrm{a}}$ of two-faced guest with

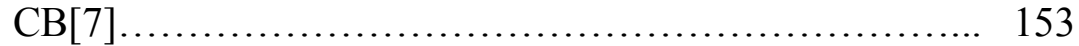

4.4.4 UV/Vis Experiments....................................... 154

4.4.4.1 Determination of Enzymatic Activity of Acetylcholinesterase............................... 154

4.4.5 Fluorescence Spectroscopy............................... 159 4.4.5.1 Measurement of the Binding Constant of the Inhibitor to BCA using Fluorescence Spectroscopy............... 160

4.4.5.2 Measurement of the Dissociation Constant of Different Sulfonamides from BCA............................ 163

V Chapter 5: Summary and Future Work.

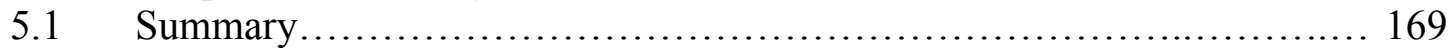

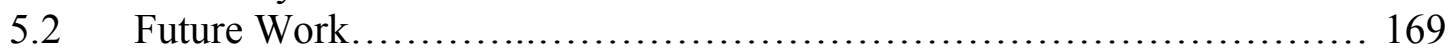

Bibliography................................................... 172 


\section{List of Tables}

\section{Chapter 1}

Table I-1. Values of $\mathrm{K}_{\mathrm{a}}\left(\mathrm{M}^{-1}\right)$ measured for the binding between $\mathrm{CB}[\mathrm{n}]$ hosts and guests $\left(\mathrm{D}_{2} \mathrm{O}, \mathrm{pD} 4.74,25^{\circ} \mathrm{C}\right)$.

Chapter 3

Table III-1: Values of $\mathrm{K}_{\mathrm{a}}\left(\mathrm{M}^{-1}\right)$ of Different Host-Guest Complexes Used In the Simulation.

Table III-2: Total Number of Self-Sorted and Non Self-Sorted States at Different Concentration.

Table III-3: Effect of Concentration of Components and Sequence of Addition on Pathways.

Table III-4: Range of binding constants of synthetic hosts used in the simulation and summary of binding constants for various interactions observed in biology.

\section{Chapter 4}

Table IV-1. Thermodynamic and Kinetic Parameters for BCA $\bullet$ Inhibitor Interactions.

Table IV-2: Thermodynamic parameters for the interaction of $\mathbf{I V - 4}$ and IV-5 with BCA and $\mathrm{CB}[7]$. 


\section{List of Figures}

\section{Chapter 1}

Figure I-1. Chemical structures of compounds used in H-bond directed self-sorting in $\mathrm{CDCl}_{3}$.

Figure I-2. Hydrogen bonding region $(8.0-14.5 \mathrm{ppm})$ of the ${ }^{1} \mathrm{H}$ NMR spectra $\left(\mathrm{H}_{2} \mathrm{O}\right.$ sat. $\left.\mathrm{CDCl}_{3}, 500 \mathrm{MHz}, 298 \mathrm{~K}\right)$ recorded for a) $\mathbf{I}-\mathbf{1 5}_{10} \cdot \mathrm{Ba}^{2+}+2$ Pic-, b) $\mathbf{I}-\mathbf{1 6}_{16} \cdot 2 \mathrm{Ba}^{2+} \cdot 4 \mathrm{Pic}-$, c) $\mathbf{I}-\mathbf{1 7}_{2}$, d) $\mathbf{I}-$

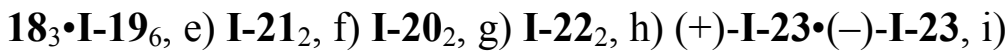
a self-sorted mixture comprising $\mathbf{I}_{-15} \mathbf{1 5}_{10} \mathrm{Ba}^{2+}+2 \mathrm{Pic}^{-}$, I$\mathbf{1 6}_{16} \cdot 2 \mathrm{Ba}^{2+} \cdot 4 \mathrm{Pic}^{-}, \mathbf{I}-\mathbf{1 7}_{2}, \mathbf{I}-\mathbf{1 8}_{3} \cdot \mathbf{I}-\mathbf{1 9}_{6}, \mathbf{I}-\mathbf{2 1}_{2}, \mathbf{I}-\mathbf{2 0}_{2}, \mathbf{I}-\mathbf{2 2}{ }_{2}$, and $(+)-\mathbf{I}-23 \bullet(-)-\mathbf{I}-23$. The representations depict the species present in solution. The resonances are color coded to aid comparison.

Figure I-3. Chemical structures of compounds used for social selfsorting in aqueous solution driven by ion-dipole, metalligand, $\pi-\pi$, and hydrophobic interactions.

Figure I-4. Chemical structures of four of the ten conformational isomers of I-44. Arrows highlight the four two-fold rotors. 


\section{Chapter 2}

Figure II-1. Schematic representation of three members of the conformational ensemble open to dimeric molecular clips driven solely by $\pi-\pi$ interactions: a) perpendicular geometry, b) skewed geometry, and c) in register geometry.

Figure II-2. Portion of the ${ }^{1} \mathrm{H}$ NMR (500 MHz, RT) spectrum recorded for: a) II-2 in DMSO- $d_{6}$, b) II-2•II-2 in $\mathrm{CDCl}_{3}$, c) $( \pm)-\mathbf{I I}-\mathbf{5}$ in DMSO- $\left.d_{6}, \mathrm{~d}\right)(+)-\mathbf{I I - 5} \cdot(-)-\mathbf{I I - 5}$ in $\mathrm{CDCl}_{3}$.

Figure II-3. Portion of the variable concentration ${ }^{1} \mathrm{H}$ NMR spectrum recorded for a $\left(400 \mathrm{MHz}, \mathrm{CDCl}_{3}, \mathrm{RT}\right)$ : a) $5 \mathrm{mM}$ of II-2•II2, b) $50 \mu \mathrm{M}$ of II-2•II-2, c) $5 \mathrm{mM}$ of (+)-II-5•(-)-II-5 and d) $50 \mu \mathrm{M}$ of (+)-II-5•(-)-II-5.

Figure II-4. Schematic representation of geometrical match versus geometrical mismatch: a) II-1•II-1 and b) (+)-II-4a•(-)-II4a, c) hypothetical diastereomeric aggregate formed by $( \pm)-$ $\mathbf{I I - 1} \cdot \mathbf{I I}-\mathbf{4 a}$, and d) racemic heterodimer $( \pm)-\mathbf{I I}-\mathbf{1} \bullet \mathbf{I I}-\mathbf{2}$.

Figure II-5. Portion of the ${ }^{1} \mathrm{H}$ NMR $\left(2.5 \mathrm{mM}, 500 \mathrm{MHz}, \mathrm{CDCl}_{3}, \mathrm{RT}\right)$ spectrum recorded for: a) $\mathbf{I I - 1} \cdot \mathbf{I I}-\mathbf{1}$, b) $\mathbf{I I}-\mathbf{2} \cdot \mathbf{I I}-\mathbf{2}$, and c) a mixture of II-1•II-1, II-2•II-2, and ( $\mathbf{\pm})-\mathbf{I I}-\mathbf{1} \bullet \mathbf{I I}-\mathbf{2}$. 
Figure II-6. Cross-eyed stereoviews of the molecular structures of: a)

II-1•II-1, b) II-2•II-2, c) (+)-II-4b•(-)-II-4b, and d) (+)-II5•(-)-II-5 in the crystal. Color coding: C, gray; H, white; $\mathrm{N}$, blue; O, red; H-bonds, yellow-red striped.

Figure II-7. Cross-eyed stereoviews of the molecular structures of: II8•II-8 in the crystal. Color coding: C, gray; H, white; N, blue; O, red; H-bonds, yellow-red striped.

Figure II-8. Portion of the ${ }^{1} \mathrm{H}$ NMR (500 MHz, RT) spectrum recorded for : a) II-7 in DMSO- $d_{6}$, b) II-7-II-7 in $\mathrm{CDCl}_{3}$.

Figure II-9. Portion of the ${ }^{1} \mathrm{H}$ NMR spectrum recorded for II-7•II-7 (400 $\left.\mathrm{MHz}, \mathrm{CDCl}_{3}, \mathrm{RT}\right)$ : a) $10 \mathrm{mM}$, and b) $50 \mu \mathrm{M}(\mathrm{x}=$ $\left.{ }^{13} \mathrm{CHCl}_{3}\right)$

Figure II-10. Schematic representation of the shapes of II-7・II-7: a) steric interaction in the head-to-head form, and b) a skewed geometry that avoids steric interactions.

Figure II-11. ${ }^{1} \mathrm{H}$ NMR spectra (500 MHz, $\left.298 \mathrm{~K}\right)$ for: a) II-2•II-2, b) (+)II-5•(-)- II-5, c) II-7•II-7, and d) a mixture of II-2•II-2, (+)-II-5•(-)-II-5, and II-7•II-7.

Figure II-12. ${ }^{1} \mathrm{H}$ NMR spectra (400 MHz, $\left.298 \mathrm{~K}\right)$ for: a) (+)-II-5•(-)-II5, b) II-1• II-1, c) II-7•II-7, and d) a mixture of II-1・II-1, (+)-II-5•(-)-II-5, and II-7•II-7. 
Figure II-13. ${ }^{1} \mathrm{H}$ NMR spectra $(500 \mathrm{MHz}, 298 \mathrm{~K})$ for: a) a mixture of II2•II-2, (+)-II-5•(-)-II-5, and II-7•II-7, and b) selective heterodimerization of II-2•II-2 under the formation of II1•II-2 upon addition of II-1•II-1.

Figure II-14. Portion of the ${ }^{1} \mathrm{H}$ NMR spectra recorded for $9 \bullet 9(400 \mathrm{MHz}$, $\mathrm{CDCl}_{3}$, RT) upon dilution: a) $11 \mathrm{mM}$, b) $1.1 \mathrm{mM}$, and c) $0.11 \mathrm{mM}$. Resonances are color coded as follows: dimeric II-8•II-8, red; and monomeric II-8, blue.

Figure II-15. Portion of the variable temperature ${ }^{1} \mathrm{H}$ NMR spectrum recorded for II-8•II-8 (400 MHz, $\left.2.5 \mathrm{mM}, \mathrm{CDCl}_{3}, \mathrm{RT}\right)$ : a) $18^{\circ} \mathrm{C}$, b) $31^{\circ} \mathrm{C}$, c) $41^{\circ} \mathrm{C}$, and d) $55^{\circ} \mathrm{C}$. Resonances are color coded as follows: dimeric $\mathbf{I I - 8} \cdot \mathbf{I I - 8}$, red; and monomeric II-8, blue.

Figure II-16. Portion of the variable concentration ${ }^{1} \mathrm{H}$ NMR spectrum recorded for 9 (400 MHz, $\left.\mathrm{CDCl}_{3}, \mathrm{RT}\right)$ : a) $10 \mathrm{mM}$, b) 1.0 $\mathrm{mM}$, and c) $0.10 \mathrm{mM}$.

Figure II-17. ${ }^{1} \mathrm{H}$ NMR spectra (400 MHz, 298 K) for: a) II-9, b) II-8•II$\mathbf{8}$ in equilibrium with II-8, and c) a mixture of II-8-II-8 in equilibrium with II-8, and II-9. 
Figure II-18. Molecular clips that remain monomeric in solution: a) Steric bulk prevents dimeric aggregation of II-9, and b) Crystal structure of $( \pm)$-II-11 showing the display of Hbonding groups in front of the cleft which leads to steric hindrance to dimerization.

Figure II-19. Portion of the variable concentration ${ }^{1} \mathrm{H}$ NMR spectrum recorded for ( \pm )-II-11 (400 $\left.\mathrm{MHz}, \mathrm{CDCl}_{3}, \mathrm{RT}\right)$ : a) $10 \mathrm{mM}$, b) $1.0 \mathrm{mM}$, and c) $0.10 \mathrm{mM}$.

Figure II-20. Schematic representation of the aggregates formed by a) II1・II-1, b) II-3•II-3 and c) ( \pm )-II-6.

Figure II-21. Portion of the variable concentration ${ }^{1} \mathrm{H}$ NMR spectrum recorded for ( \pm )-II-6 (400 MHz, $\left.\mathrm{CDCl}_{3}, \mathrm{RT}\right)$ : a) $15.51 \mathrm{mM}$, b) $9.30 \mathrm{mM}$, and c) $5.58 \mathrm{mM}$, d) $2.23 \mathrm{mM}$, e) $1.11 \mathrm{mM}$, f) $0.89 \mathrm{mM}, \mathrm{g}) 0.55 \mathrm{mM}, \mathrm{h}) 0.35 \mathrm{mM}$, i) $0.27 \mathrm{mM}$.

Figure II-22. Portion of the ${ }^{1} \mathrm{H}$ NMR (2 mM, $\left.500 \mathrm{MHz}, \mathrm{CDCl}_{3}, \mathrm{RT}\right)$ spectrum recorded for: a) (+)-II-5•(-)-II-5, b) ( \pm )-II-6, and c) a mixture of $(+)-\mathbf{I I}-5 \bullet(-)-\mathbf{I I}-\mathbf{5}$, and $( \pm)-\mathbf{I I}-\mathbf{6}$.

Figure II-17. ${ }^{1} \mathrm{H}$ NMR spectra (400 MHz, $\left.298 \mathrm{~K}\right)$ for: a) II-2•II-2, b) (+)-II-5•(-)-II-5, c) II-8 in equilibrium with II-8•II-8, d) II-9, and e) a mixture of II-2•II-2, (+)-II-5•(-)-II-5, II-8 in equilibrium with II-8・II-8, and II-9. 


\section{Chapter 3}

Figure III-1. ${ }^{1} \mathrm{H}$ NMR spectra (400 MHz, $\left.\mathrm{D}_{2} \mathrm{O}, \mathrm{pD} 7.4,298 \mathrm{~K}, 1 \mathrm{mM}\right)$ recorded for: A) III-6•III-1, B) III-5•III-2, C) III-7•III-3, D) III-8•III-4, and E) III-6•III-1, III-5•III-2, III-7•III-3, and III-8•III-4. $\left(\mathrm{CD}_{3}\right)_{3} \mathrm{SiCD}_{2} \mathrm{CD}_{2} \mathrm{CO}_{2} \mathrm{D}(\Delta)$ is used as internal standard.

Figure III-2. ${ }^{1} \mathrm{H}$ NMR spectra (400 MHz, $\left.\mathrm{D}_{2} \mathrm{O}, \mathrm{pD} 7.4,298 \mathrm{~K}, 1 \mathrm{mM}\right)$ recorded for an equimolar mixture after addition of 1 eq. of: A) III-1, B) III-8, C) III-3, D) III-5, E) III-4, F) III-7, G) III-2, H) III-6. $\left(\mathrm{CD}_{3}\right)_{3} \mathrm{SiCD}_{2} \mathrm{CD}_{2} \mathrm{CO}_{2} \mathrm{D}(\Delta)$ is used as internal standard. Prime indicates that the guest is complexed with host.

Figure III-3. Schematic representation of the network in stepwise formation of a self-sorted mixture comprising: a) two, b) three, and c) four components.

Figure III-4. Schematic representation of 2, 3, 4, 6, and 8-dimensional hypercubes. 
Figure III-5. Stepwise construction of a four component self-sorting mixture: (a) equilibria considered, (b) concentrations of the components, (c) association constants of the various complexes, and (d) a plot of free energy versus number of components.

Figure III-6. A pathway that proceeds through eight self-sorted states created by adding all the hosts (III-5 - III-8) in the first four steps followed by the guests (III-4, III-3, III-2, then III-1) in the next four steps.

Figure III-7. A pathway created by the alternate addition of hosts and guests that proceeds through some self-sorted states.

Figure III-8. Stepwise construction of an 8-component self-sorting mixture (concentration of each component $=1 \mathrm{M})$ : a) all 256 possible states (color coding: self-sorted states, green; non-self-sorted states, red.), b) plot of all 40320 pathways, c) steps that transform a self-sorted state to another selfsorted state, d) steps that transform a non self-sorted state to another non self-sorted state, e) steps that transform a non self-sorted state to another self-sorted state, and f) steps that transform a self-sorted state to another non self-sorted state. 
Figure III-9. A plot showing the change in number of self-sorted states with respect to concentration.

Figure III-10. All 576 pathways for each of the following order of addition (concentration of each component $=1 \mathrm{M}$ ): (a) GGGGHHHH, (b) HHHHGGGG, (c) GHGHGHGH, and (d) HGHGHGHG.

Figure III-11. A histogram showing the distribution of 17152 states originated from randomly generated binding constants for 67 systems similar to experimental system.

Figure III-12. A stack of ten simulations from randomly generated binding constants showing that only two cases the final state is self-sorted. (states are color coded as follows: selfsorted states, green dots; non self-sorted states, red dots.)

\section{Chapter 4}

Figure IV-1. a) A plot of change in absorbance versus time for IV-2: Step $1(\bigcirc)$, Step $2(\times) ;$ Step $3(\bullet)$; Step $4(\triangle)$; Step $5(\square)$; Step $6(\diamond)$; Step $7(\mathbf{\Delta})$; and Step $8(\nabla)$. b) A plot of relative activity of BCA versus number of steps. 
Figure IV-2. ${ }^{1} \mathrm{H}$ NMR spectra recorded for $\left(400 \mathrm{MHz}, 30 \mathrm{mM} \mathrm{NaD} \mathrm{PO}_{4}\right.$ Buffer, pD 7.3, $298 \mathrm{~K})$ : a) BCA (1 eq.), b) BCA (1 eq.) and IV-2 (1.76 eq.), c) BCA (1 eq.), IV-2 (1.76 eq.) and CB[7] (0.5 eq.), d) BCA (1 eq.), IV-2 (1.76 eq.) and CB[7] (2.0 eq.), and e) IV-2 (2 eq.) and CB[7] (1 eq.).

Figure IV-3. Plot of fluorescence intensity versus [IV-1] in the titration of BCA $\bullet I V-12$ with IV-1. Concentration of BCA $(67.5$ $\mathrm{nM})$, IV-12 $(2 \mu \mathrm{M})$, and IV-1 (50 $\mathrm{nM}-10 \mu \mathrm{M}) . \quad$ All measurements were done in $20 \mathrm{mM} \mathrm{NaH}{ }_{2} \mathrm{PO}_{4}$ Buffer, pH $7.3,298 \mathrm{~K}$.

Figure IV-4. Plot of fluorescence intensity versus time after addition of $\mathrm{CB}[7](50 \mu \mathrm{M})$ to a mixture of IV-12 $(2 \mu \mathrm{M}), \mathbf{I V}-\mathbf{1}(20$ $\mu \mathrm{M})$ and BCA (80 $\mathrm{nM})$. All measurements were done in $20 \mathrm{mM} \mathrm{NaH}_{2} \mathrm{PO}_{4}$ buffer, $\mathrm{pH} 7.3,298 \mathrm{~K}$.

Figure IV-5. Plot of fluorescence intensity versus time: a) Addition of IV-12 to a solution of BCA and IV-12. Final concentration of BCA, IV-12, and IV-1 are $76 \mathrm{~nm}, 98 \mu \mathrm{M}$ and $2 \mu \mathrm{M}$. b) addition of $\mathrm{CB}[7]$ to a solution containing BCA, IV-12, and IV-1. Final concentrations of BCA, IV-12, IV-2 and $\mathrm{CB}[7]$ are $75 \mathrm{nM}, 25 \mu \mathrm{M}, 18 \mu \mathrm{M}$ and $50 \mu \mathrm{M}$, respectively. All experiments were done in $20 \mathrm{mM} \mathrm{NaH}{ }_{2} \mathrm{PO}_{4}$ Buffer, $\mathrm{pH}$ $7.3,298 \mathrm{~K}$. 
Figure IV-6. a) Titration of AChE with IV-4, and b) Lineweaver-Burk plot of AChE activity in the presence of IV-4.

Figure IV-7. a) Fluorescence spectrum of a solution containing $\mathrm{CB}[7] \cdot \mathbf{I V}-\mathbf{1 1}$ and b) after addition of the stock solution containing AChE $\bullet I V-4$ to a mixture of CB[7] $\bullet \mathbf{I V - 1 1 . ~}$

Figure IV-8. A plot of the rate of hydrolysis of IV-6 by AChE versus concentration of $\mathrm{CB}[7]$.

Figure IV-9: Sample Determination of the binding constant of IV-1

Figure IV-10. Plot of rate of hydrolysis versus concentration of acetylthiocholine.

Figure IV-11. a) Plot of $\alpha$ versus [IV-4]; and b) Plot of $\alpha^{\prime}$ versus [IV-4].

Figure IV-12. Lineweaver-Burk plot of AChE activity in the presence of

$$
\text { IV-5. }
$$

Figure IV-13. Plot of fluorescence intensity versus wavelength in the titration of a solution of BCA by $\mathbf{I V - 1 2}\left(20 \mathrm{mM} \mathrm{NaH}_{2} \mathrm{PO}_{4}\right.$ Buffer, pH 7.3, 298 K).

Figure IV-14. Determination of the $\mathrm{K}_{\mathrm{a}}$ value of $\mathbf{I V - 1 2}$ by nonlinear leastsquares analysis fitting to a 1:1 binding model. 
Figure IV-15. Plot of fluorescence intensity versus [IV-2] in the titration of BCA $\bullet I V-12$ with IV-2. Concentration of BCA (67.5 $\mathrm{nM})$, IV-12 $(2 \mu \mathrm{M})$, and IV-2 $(50 \mathrm{nM}-10 \mu \mathrm{M}) . \quad$ All measurements were done in $20 \mathrm{mM} \mathrm{NaH} \mathrm{PO}_{4}$ Buffer, pH $7.3,298 \mathrm{~K}$.

Figure IV-16. Plot of fluorescence intensity versus $[\mathrm{CB}[7] \cdot \mathbf{I V}-\mathbf{1}]$ in the titration of BCA $\bullet \mathbf{I V - 1 2}$ with $\mathrm{CB}[7] \cdot \mathbf{I V}-\mathbf{1}$. Concentration of BCA $(86 \mathrm{nM}), \mathbf{I V}-12(2 \mu \mathrm{M})$, and CB[7] $\mathbf{I V}-\mathbf{1}(2.5 \mathrm{nM}-$ $120 \mu \mathrm{M})$. All measurements were done in $20 \mathrm{mM}$ $\mathrm{NaH}_{2} \mathrm{PO}_{4}$ Buffer, pH 7.3, $298 \mathrm{~K}$.

Figure IV-17. Plot of fluorescence intensity versus $[\mathrm{CB}[7] \cdot \mathbf{I V}-\mathbf{2}]$ in the titration of BCA $\bullet \mathbf{I V - 1 2}$ with $\mathrm{CB}[7] \cdot \mathbf{I V}-\mathbf{2}$. Concentration of BCA $(86 \mathrm{nM}), \mathbf{I V}-\mathbf{1 2}(2 \mu \mathrm{M})$, and CB[7] $\mathbf{I V - 2}(6.5 \mathrm{nM}-$ $400 \mu \mathrm{M})$. All measurements were done in $20 \mathrm{mM}$ $\mathrm{NaH}_{2} \mathrm{PO}_{4}$ Buffer, $\mathrm{pH} 7.3,298 \mathrm{~K}$.

Figure IV-18. Plot of fluorescence intensity at $460 \mathrm{~nm}$ versus time after addition of IV-12 (1.5 $\mu \mathrm{M}-98 \mu \mathrm{M})$ to a solution containing BCA $(80 \mathrm{nM})$ and IV-1 $(2 \mu \mathrm{M})(20 \mathrm{mM}$ $\mathrm{NaH}_{2} \mathrm{PO}_{4}$ Buffer, pH 7.3, $\left.298 \mathrm{~K}\right)$. 
Figure IV-19. Plot of $\mathrm{k}_{\mathrm{obs}}$ for the dissociation of $\mathbf{I V}-\mathbf{1}$ from BCA versus concentration of IV-12. (20 mM NaH${ }_{2} \mathrm{PO}_{4}$ Buffer, $\mathrm{pH} 7.3$, $298 \mathrm{~K})$.

Figure IV-20. Plot of fluorescence intensity at $460 \mathrm{~nm}$ versus time after addition of IV-12 $(98 \mu \mathrm{M})$ to a solution containing BCA $(80 \mathrm{nM})$ and IV-1 $(2 \mu \mathrm{M})\left(20 \mathrm{mM} \mathrm{NaH} \mathrm{PO}_{4}\right.$ Buffer, pH $7.3,298 \mathrm{~K})$.

Figure IV-21. Plot of $\mathrm{k}_{\mathrm{obs}}$ for the dissociation of IV-2 from BCA versus concentration of IV-12. (20 mM NaH${ }_{2} \mathrm{PO}_{4}$ Buffer, $\mathrm{pH}$ 7.3, $298 \mathrm{~K})$.

Figure IV-22. Plot of fluorescence intensity at $460 \mathrm{~nm}$ versus time after addition of IV-12 $(98 \mu \mathrm{M})$ to a solution containing BCA $(80 \mathrm{nM})$ and IV-2 $(2 \mu \mathrm{M})\left(20 \mathrm{mM} \mathrm{NaH} \mathrm{PO}_{4}\right.$ Buffer, $\mathrm{pH}$ $7.3,298 \mathrm{~K})$.

Figure IV-23. Plot of $\mathrm{k}_{\mathrm{obs}}$ for the dissociation of IV-12 from BCA versus concentration of 1. (20 mM NaH $\mathrm{PO}_{4}$ Buffer, $\mathrm{pH} 7.3,298$ K).

Figure IV-24. Plot of fluorescence intensity at $460 \mathrm{~nm}$ versus time after addition of IV-1 $(75 \mu \mathrm{M})$ to a solution containing BCA (80 $\mathrm{nM})$ and IV-12 $(2 \mu \mathrm{M})\left(20 \mathrm{mM} \mathrm{NaH} \mathrm{PO}_{4}\right.$ Buffer, $\mathrm{pH}$ 7.3, $298 \mathrm{~K})$. 
Figure IV-25. a) Plot of fluorescence intensity versus time after addition of $\mathrm{CB}[7]$ to a mixture of $\mathbf{I V - 1 2}, \mathbf{I V}-\mathbf{1}$ and BCA. Final concentrations of BCA, IV-12, IV-1 and CB[7] are $75 \mathrm{nM}$, $25 \mu \mathrm{M}, 18 \mu \mathrm{M}$, and $50 \mu \mathrm{M}$ respectively; All measurements were done in $20 \mathrm{mM} \mathrm{NaH}_{2} \mathrm{PO}_{4}$ Buffer, $\mathrm{pH} 7.3,298 \mathrm{~K}$.

Figure IV-26. a) Plot of fluorescence intensity versus time after addition of $\mathrm{CB}[7]$ to a mixture of $\mathbf{I V - 1 2}, \mathbf{I V}-\mathbf{2}$ and BCA. Final concentrations of BCA, IV-12, IV-2 and CB[7] are $73 \mathrm{nM}$, $25 \mu \mathrm{M}, 18 \mu \mathrm{M}$, and $50 \mu \mathrm{M}$ respectively; All measurements were done in $20 \mathrm{mM} \mathrm{NaH}_{2} \mathrm{PO}_{4}$ Buffer, $\mathrm{pH} 7.3,298 \mathrm{~K}$.

Figure IV-27. A comparison of decrease in fluorescence intensity of a solution containing IV-12 $(2 \mu \mathrm{M})$ and BCA $(80 \mathrm{nM})$ in presence of IV-1 $(20 \mu \mathrm{M})$ and CB[7]• IV-1 $(20 \mu \mathrm{M})$.

Figure IV-28. a) Plot of fluorescence intensity versus time after addition of $\mathrm{CB}[7]$ to a mixture of IV-12, IV-2 and BCA. Final concentrations of BCA, IV-12, IV-2 and CB[7] are $80 \mathrm{nM}$, $2 \mu \mathrm{M}, 20 \mu \mathrm{M}$, and $50 \mu \mathrm{M}$ respectively; and b) $\mathrm{A}$ comparison of rate of dissociation of IV-12 $(2 \mu \mathrm{M})$ from BCA $(80 \mathrm{nM})$ in presence of $\mathbf{I V - 2}(20 \mu \mathrm{M})$ and CB[7]•IV$2(20 \mu \mathrm{M})$. 


\section{List of Schemes}

\section{Chapter 1}

Scheme I-1. Self-recognition within mixtures of bipyridine based $\mathrm{Cu}^{+}$ double helicates.

Scheme I-2. Self-recognition of two bipyridine trimers in presence of $\mathrm{Cu}^{+}$and $\mathrm{Ni}^{2+}$.

Scheme I-3. Self-sorting based on thermodynamically controlled transesterification during macrocyclization of predisposed building blocks.

Scheme I-4. Stoichiometry induced partner displacement in a fourcomponent mixture: (a) equilibria considered, (b) constraints imposed, (c) mole fraction definitions, and (d) a plot of mole fraction versus guest concentration $\left(\left[\mathrm{M}_{\mathrm{tot}}\right]=\right.$ $\left.\left[\mathrm{N}_{\text {tot }}\right]\right)$.

Scheme I-5. Enantiomeric self-recognition of ( \pm )-I-11 is triggered by addition of I-12.

Scheme I-6. Self-sorting behavior of C-shaped molecular clips I-13e and ( $( \pm)-\mathbf{I}-14 \mathbf{e}$.

Scheme I-7. Illustration of the competition between a single guest for different sized $\mathrm{CB}[\mathrm{n}]$. 
Scheme I-8. Kinetic and thermodynamic self-sorting based on guests with two binding epitopes.

Scheme I-9. The sequential addition of various $\mathrm{CB}[\mathrm{n}]$ and guests to I-44 induces folding, forced unfolding, and refolding of I-44 into four different conformations.

Scheme I-10. Homochiral self-sorting assembly of meso-linked porphyrin $\mathbf{I}-45$.

Scheme I-11. Hexameric assemblies based on DDA-AAD H-bonding motif.

Scheme I-12. Mixed aggregates formed from mixing of $(\mathbf{I}-46-\text { G1 })_{6}$ and $(\mathbf{I}-\mathbf{4 6}-\mathbf{G 3})_{6}$.

Scheme I-13. Homodimer formation by fluorous and hydrocarbon core peptides.

Scheme I-14. Self-sorting within amphiphilic peptides driven by steric matching and electrostatic effects.

Scheme I-15. Formation of a dynamic imine library and $\mathrm{Cu}^{+}$templated self-sorting.

Scheme I-16. Self-sorting on patterned surfaces.

Scheme I-17. Geometric self-sorting of DNA nanostructures. 
Scheme I-18. Self-sorting non-covalent functionalization of a universal polymer backbone.

Scheme I-19. Self-sorting in dendritic peptide gels based on head group size or chirality.

\section{Chapter 2}

Scheme II-1. Synthesis of molecular clips II-1 - II-11. Conditions: a) $\mathrm{Pd} / \mathrm{C}, \mathrm{H}_{2}, \mathrm{DMF}$, b) $\mathrm{PhNCO}$ or R'COCl, $\mathrm{Et}_{3} \mathrm{~N}, \mathrm{CH}_{2} \mathrm{Cl}_{2}$, c) II-14, $t$-BuOK, DMSO, d) PTSA, $\left(\mathrm{CH}_{2} \mathrm{O}\right)_{n}, \mathrm{ClCH}_{2} \mathrm{CH}_{2} \mathrm{Cl}$, reflux, e) $p$-xylene, reflux, f) TFA, $\mathrm{HNO}_{3}$.

\section{Chapter 4}

Scheme IV-1. Thermodynamic Shuttling of IV-1 between BCA and $\mathrm{CB}[7]$.

Scheme IV-2. Synthesis of IV-1 - IV-5. Conditions: a) $\mathrm{K}_{2} \mathrm{CO}_{3}, \mathrm{CH}_{3} \mathrm{CN}$, reflux, b) $\mathrm{RNH}_{2}, \mathrm{~K}_{2} \mathrm{CO}_{3}, \mathrm{KI}$, anh. THF, reflux, c) $140{ }^{\circ} \mathrm{C}$, neat.

Scheme IV-3. Chemical Reactions Used to Monitor Enzymatic Activities.

Scheme IV-4. Schematic Representation of the Fluorophore Displacement Assay.

Scheme IV-5. Proposed Mechanism of CB[7] Assisted Dissociation of IV-1 and IV-2 from BCA. 
Scheme IV-6. A Complete Shuttling of IV-2 Between BCA and CB[7].

Scheme IV-7. Formation of Ternary Complex Upon Addition of CB[7] to $\mathrm{AChE} \cdot \mathbf{I V}-4$

\section{Chapter 5}

Scheme V-1. Self-sorting of polymer bound molecular clips.

Scheme V-2. Selective regulation of the activity of an enzyme in a mixture. 


\section{List of Abbreviations}

Ac

AFM

anh.

aq.

br. s

$t-\mathrm{Bu}$

calcd

conc

CPK

d

dd

dec

DMSO

DNA

EI

EtOAc

FAB

$\mathrm{h}$

HR-MS

$\mathrm{Hz}$

IR

$J$ acetyl

atomic force microscopy

anhydrous

aqueous

broad singlet

tertiary butyl

calculated

concentration

Corey, Pauling and Koltun

doublet

doublet of doublets

decomposition

dimethyl sulfoxide

deoxyribonucleic acid

electron ionization

ethyl acetate

fast atom bombardment

hour(s)

high resolution mass spectroscopy

hertz

infrared

coupling constant 


\begin{tabular}{|c|c|}
\hline $\mathrm{m}$ & multiplet \\
\hline$m$ & meta \\
\hline $\mathrm{M}^{+}$ & molecular ion \\
\hline$m / z$ & mass-to-charge ratio \\
\hline $\mathrm{MHz}$ & megahertz \\
\hline $\min$ & minute(s) \\
\hline M.p. & melting point \\
\hline MS & mass spectroscopy \\
\hline NMR & nuclear magnetic resonance \\
\hline$o$ & ortho \\
\hline OAc & acetate \\
\hline$p$ & para \\
\hline $\mathrm{Ph}$ & phenyl \\
\hline PTSA & $p$-toluenesulfonic acid \\
\hline$q$ & quartet \\
\hline$R_{\mathrm{f}}$ & retention factor \\
\hline RT & room temperature \\
\hline $\mathrm{S}$ & second(s) \\
\hline $\mathrm{t}$ & triplet \\
\hline TFA & trifluoroacetic acid \\
\hline TLC & thin layer chromatography \\
\hline TMS & trimethylsilyl \\
\hline
\end{tabular}




\section{Chapter 1: Literature Review of Complex Self-Sorting Systems $^{1}$}

\subsection{Introduction.}

Beginning with the pioneering work of Cram, Lehn, and Pederson which defined the area of supramolecular chemistry as a contemporary discipline, the chemical community has focused significant attention on elucidating the fundamental aspects of non-covalent interactions between molecules. ${ }^{2-4}$ For example, the use of $\mathrm{H}-$ bonds, $\pi-\pi$, and metal-ligand interactions as the driving force for the build up of complex structures under thermodynamic control is now relatively well developed. ${ }^{5-9}$ Accordingly, in the past decade the emphasis in the supramolecular chemistry has shifted toward the development of self-assembled systems whose function derives from the precise orientation of the components relative to one another. For example, chemists have developed molecular machines that rely on intra-aggregate movements, chemical sensors that function by changes in the UV/Vis or fluorescence output of a chromophore upon aggregation, and membrane transporters that shuttle ions and molecules across the hydrophobic biological interface. ${ }^{10-13}$ All of these functional systems rely on the design and a priori synthesis of specific molecules (e.g. hosts, chromophores, machines, transporters) with specific structural features for specific function. 


\subsection{Self-Sorting}

Self-sorting refers to the ability of a molecule to efficiently distinguish between self- and non-self even with a complex mixture. As such the concept of selfsorting is intimately tied to the concepts of binding affinity, binding selectivity, and multi-component mixtures that are so commonly encountered in biological systems.

\subsubsection{Early Examples of Self-Sorting.}

In a 1993 report Lehn describes the synthesis of a series of bipyridine oligomers (I-1 - I-4) that were individually known to undergo well-defined selfassembly in the presence of $\mathrm{Cu}^{+}$by metal-ligand non-covalent interactions to afford the double helicates $\left(\mathbf{I}-\mathbf{1}_{2} \cdot \mathrm{Cu}^{+}{ }_{2}-\mathbf{I}-\mathbf{4}_{2} \cdot \mathrm{Cu}^{+}\right){ }^{14}$ Lehn then asked the simple but farreaching question of what would happen if all four oligomers where mixed together simultaneously. Would the information encoded within the molecular structure of I-1 - I-4 under the read-out of $\mathrm{Cu}^{+}$result in the formation of a simple mixture of double helicates (e.g. $\mathbf{I}-\mathbf{1}_{2} \cdot \mathrm{Cu}^{+}{ }_{2}-\mathbf{I}-\mathbf{4}_{2} \cdot \mathrm{Cu}^{+}$) by a self-recognition (self-sorting) process based on oligomer length occur or would cross-over aggregation between ligands of different length occur? In the experiment a mixture comprising $\left(\mathbf{I}-\mathbf{1}_{2} \cdot \mathrm{Cu}^{+}{ }_{2}-\mathbf{I}\right.$ $\mathbf{4}_{2} \cdot \mathrm{Cu}_{5}^{+}$) was observed (Scheme I-1). In a related experiment Lehn used the coordination number preferences of $\mathrm{Cu}^{+}$and $\mathrm{Ni}^{2+}$ to direct the self-recognition (selfsorting) of two different bipyridine trimers $\mathbf{I - 5}$ and I-6 (Scheme I-2). ${ }^{14}$ In this manner, Lehn showed that some complex mixtures can undergo surprisingly simple behavior by application of metal-ligand non-covalent interactions. 


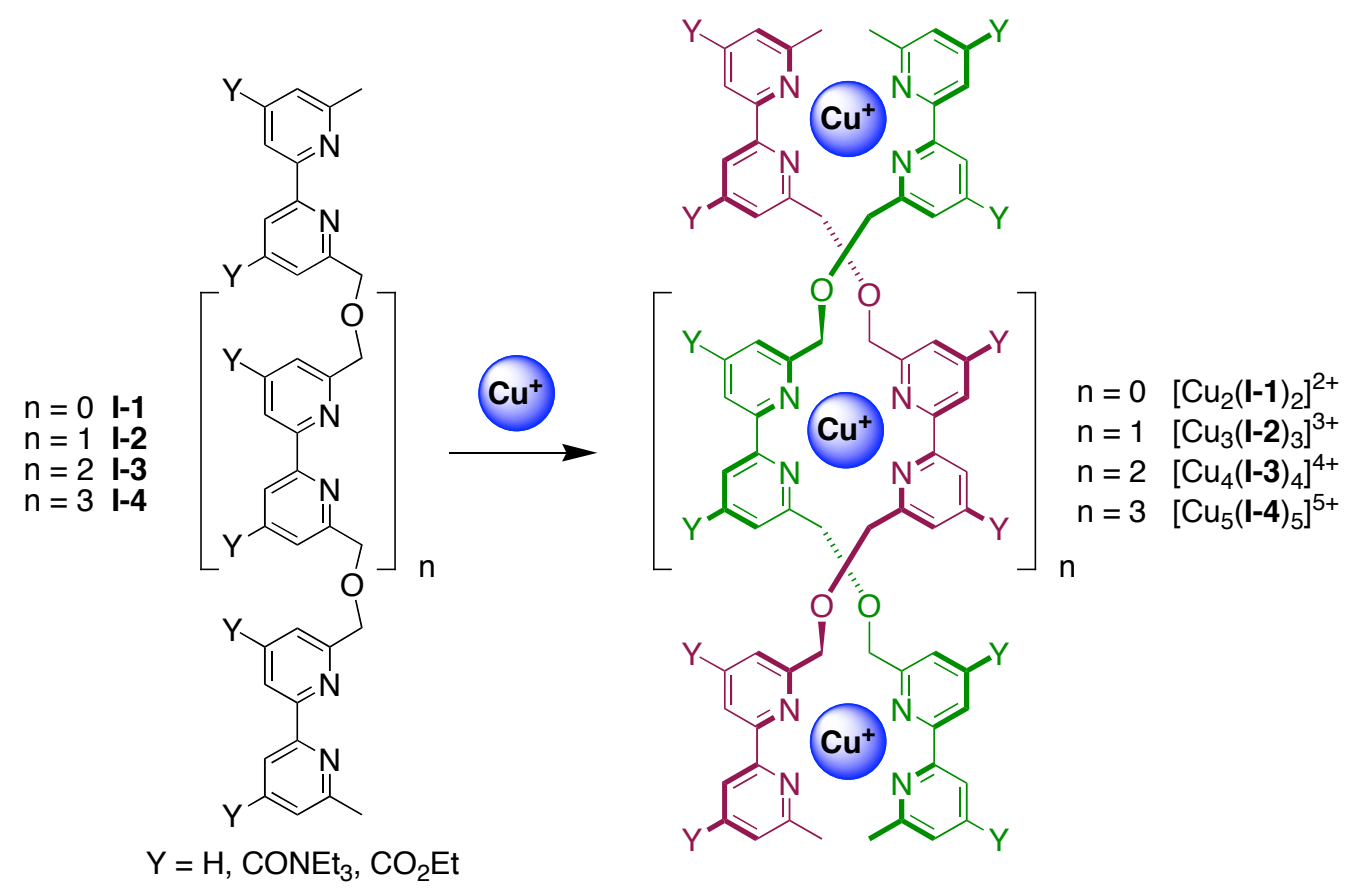

Scheme I-1. Self-recognition within mixtures of bipyridine based $\mathrm{Cu}^{+}$double helicates.
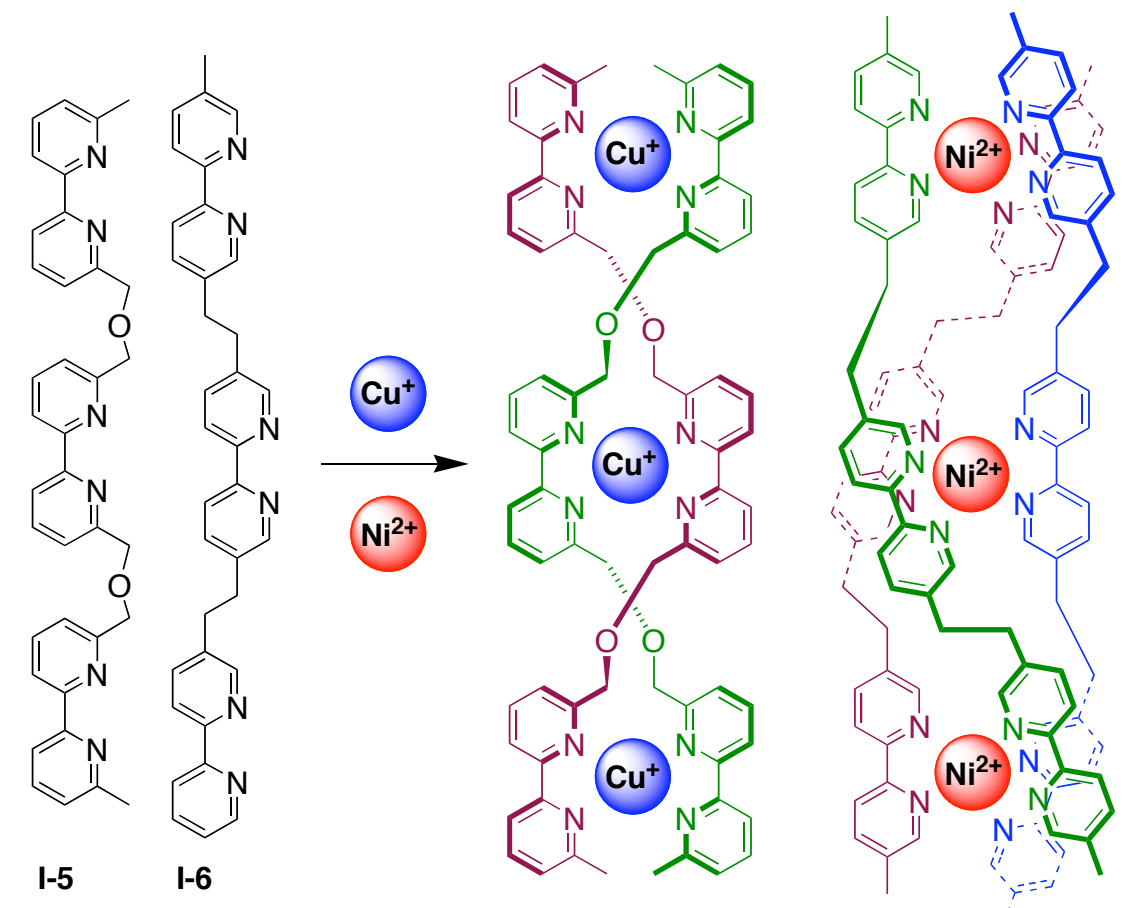

$$
\left[\mathrm{Cu}_{3}(\mathrm{I}-5)_{3}\right]^{3+} \quad\left[\mathrm{Ni}_{3}(\mathrm{I}-6)_{3}\right]^{6+i}
$$

Scheme I-2. Self-recognition of two bipyridine trimers in presence of $\mathrm{Cu}^{+}$and $\mathrm{Ni}^{2+}$. 
The first report that we are aware of in the chemical literature to use the term "self-sorting" comes from the group of Sanders. ${ }^{15}$ In this paper, Sanders reports the thermodynamically controlled trans-esterification reaction (KOMe, 18-Crown-6, toluene, reflux) of a mixture of pre-disposed building blocks I-7 and I-8 (Scheme I3). Remarkably, rather than a complex mixture of macrocycles comprising both building blocks a simple mixture of dimeric macrocycle I-10 derived from I-8 and trimeric macrocycle I-9 derived from I-7 was observed. This paper was very significant in that it began to define self-sorting as an area of research and as the first example of a self-sorting system based on reversible covalent bond formation under thermodynamic control within a mixture.

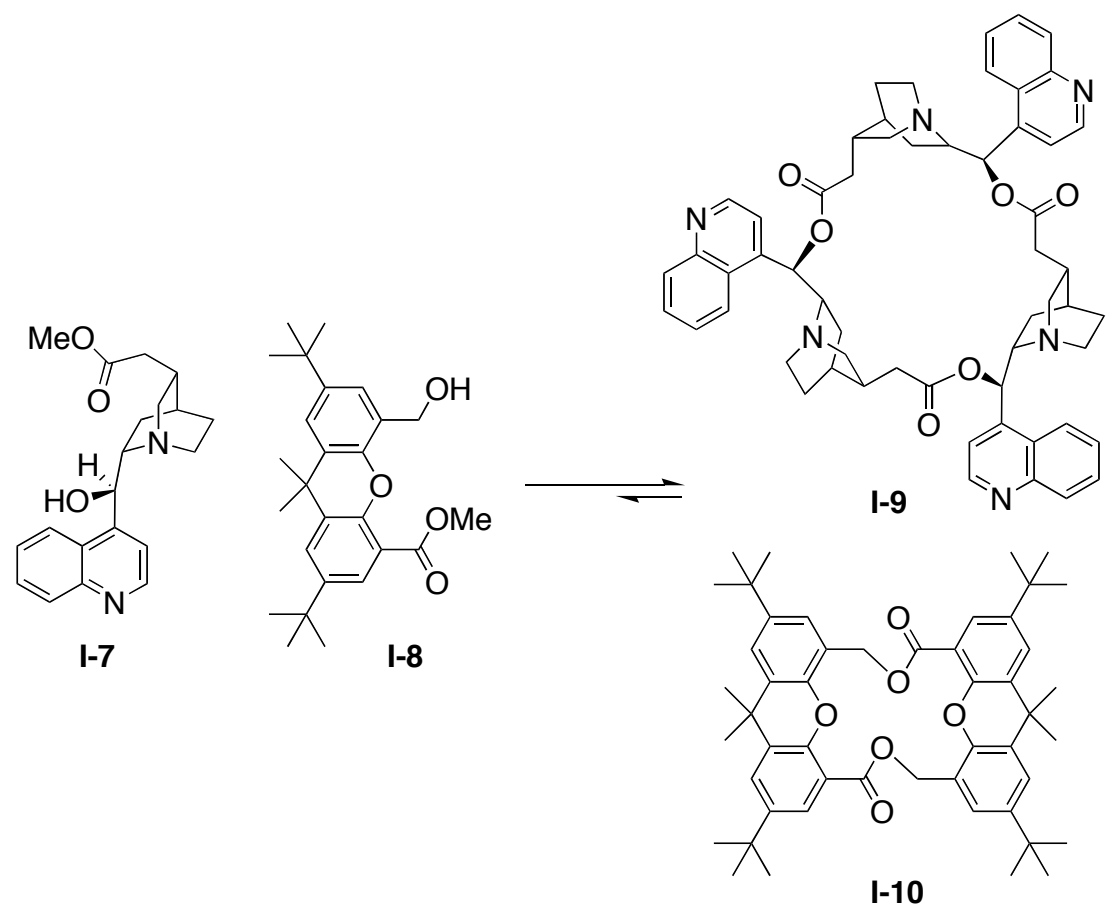

Scheme I-3. Self-sorting based on thermodynamically controlled trans-esterification during macrocyclization of predisposed building blocks. 


\subsubsection{Difference Between Self-Assembly and Self-Sorting.}

Self-assembly is the spontaneous high fidelity synthesis of a (note: singular) higher order structure from its components usually under thermodynamic control. Self-sorting refers to the spontaneous high fidelity synthesis of a system (note: plural) of higher order structures from their components usually under thermodynamic control. The critical distinction between self-assembly and self-sorting, therefore, is that self-sorting occurs within complex multi-component mixtures whereas selfassembly occurs from a single set of components.

Self-sorting systems are simply superposition of a series of well-defined aggregates from the literature. With proper design and implementation, however, it is possible to construct self-sorting systems whose behavior is different from its components. ${ }^{16}$ For example, consider the simple system comprising two hosts (A and B) and two guests ( $\mathrm{M}$ and $\mathrm{N}$ ), which can form four possible host-guest complexes (AM, AN, BM, and BN) (Scheme I-4a). We fix the total concentrations of hosts A and $\mathrm{B}\left(\left[\mathrm{A}_{\mathrm{tot}}\right]\right.$ and $\left.\left[\mathrm{B}_{\mathrm{tot}}\right]\right)$ at $1 \mathrm{mM}$ and choose the four equilibrium constants such that host A (10 $10^{4}$-fold) and host B (10-fold) both prefer guest M (Scheme I-4b). The various mole fraction definitions (Scheme I-4c) are used to construct a plot (Scheme I-4d) of the composition of the mixture as a function of total guest concentration $\left(\left[\mathrm{M}_{\mathrm{tot}}\right]=\left[\mathrm{N}_{\mathrm{tot}}\right]\right)$. When $\left[\mathrm{A}_{\mathrm{tot}}\right]=\left[\mathrm{B}_{\mathrm{tot}}\right] \geq\left[\mathrm{M}_{\mathrm{tot}}\right]=\left[\mathrm{N}_{\text {tot }}\right]$ complexes AM and BN dominate because A binds M 100-fold more tightly than B binds M. The excess free energy obtained from forming AM can be used to force $\mathrm{B}$ to accept $\mathrm{N}$ despite its individual preference for M. The critical realization is that because self-sorting systems minimize the overall free energy of the entire system, unusual behavior that differs 
from the individual components may occur. The ultimate example of this type of behavior is embodied in living systems whose multitude of components undergo well defined self-sorting processes that are orchestrated both in time and in space. No one would consider isolated proteins, nucleic acids, or lipids to be alive but few can dispute the remarkable emergent behaviors that occur when these components are present as part of the cell. The ongoing goals of our research in the self-sorting area is to go beyond those systems that are simply equal to the sum of their parts and to move toward systems that exhibit behavior similar to Nature (e.g. metastable energy dissipative rather than thermodynamically stable systems, compartmentation, catalytic events to alter the free energy landscape of complex mixtures)

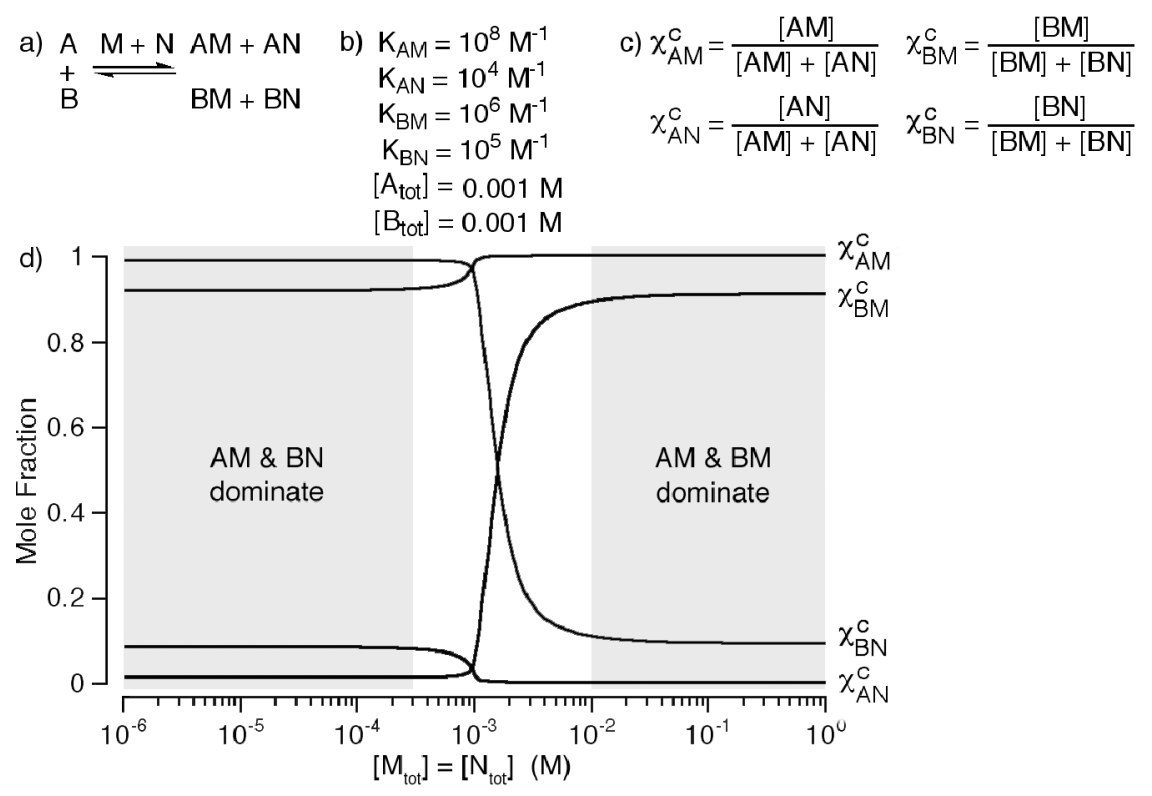

Scheme I-4. Stoichiometry induced partner displacement in a four-component mixture: (a) equilibria considered, (b) constraints imposed, (c) mole fraction definitions, and (d) a plot of mole fraction versus guest concentration $\left(\left[\mathrm{M}_{\mathrm{tot}}\right]=\left[\mathrm{N}_{\mathrm{tot}}\right]\right)$. 


\subsection{Self-Sorting Behavior of Molecular Clips}

The first report came from our group in 2002 where we used methylene bridged glycoluril dimer substructure - the fundamental building block of the cucurbit[n]uril family of macrocycles ${ }^{17-19}$ - to create a self-sorted system. We designed and synthesize $( \pm)-\mathbf{I}-11$ with the hope that it would form a square-shaped aggregate by metal-ligand interactions and the hydrophobic effect (Scheme I-5). Experimentally, we found that $( \pm)-\mathbf{I}-11$ underwent an enantiomeric self-recognition process triggered by the addition of $\mathbf{I - 1 2}$ during the formation of the racemic mixture $(+)-\mathbf{I}-11_{2} \cdot \mathbf{I}-\mathbf{1 2} 2_{2}$ and $(-)-\mathbf{I}-11_{2} \cdot \mathbf{I}-\mathbf{I 2}_{2} \cdot{ }^{20}$ The self-recognition of one enantiomer of a racemic ligand during self-assembly to form homochiral metal complexes can be viewed as a form of self-sorting (e.g. the ability to distinguish between a ligand and its enantiomer even within the more complex mixture of the two enantiomers). 


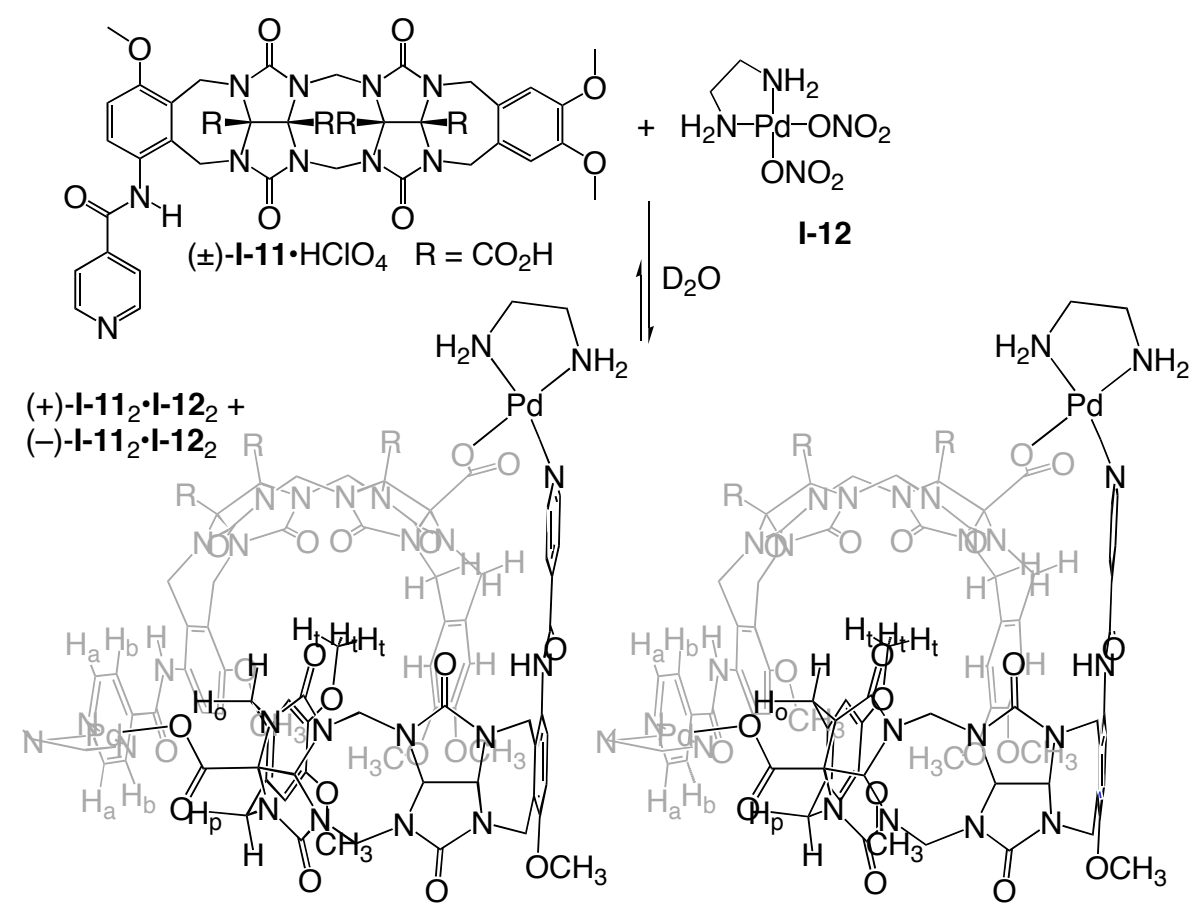

Scheme I-5. Enantiomeric self-recognition of $( \pm)$-I-11 is triggered by addition of I-

12.

Based on this result we anticipated that carboxylic acids I-13a and ( \pm )-I-14a might form even larger hydrophobically driven aggregates when combined with I-12 in water. Unfortunately, no well-defined aggregates were observed by ${ }^{1} \mathrm{H}$ NMR spectroscopy. We discovered that esters I-13e and ( \pm )-I-14e undergo tight dimerization $\left(\mathrm{K}_{\mathrm{a}} \geq 10^{6} \mathrm{M}^{-1}\right)$ in $\mathrm{CDCl}_{3}$ solution to deliver $\mathbf{I}-\mathbf{1 3} \mathbf{e}_{2}$ and (+)-I-14e•(-)-I14e by a high fidelity heterochiral recognition process (Scheme I-6). ${ }^{21}$ Naturally, we decided to investigate self- versus non-self recognition in the mixture comprising I13e and $( \pm)-\mathbf{I}-14 \mathbf{e}$ in $\mathrm{CDCl}_{3}$. ${ }^{1} \mathrm{H}$ NMR spectroscopy of this mixture was simply the superposition of the ${ }^{1} \mathrm{H}$ NMR spectra of its components, which is the spectroscopic fingerprint of a self-sorting process. 


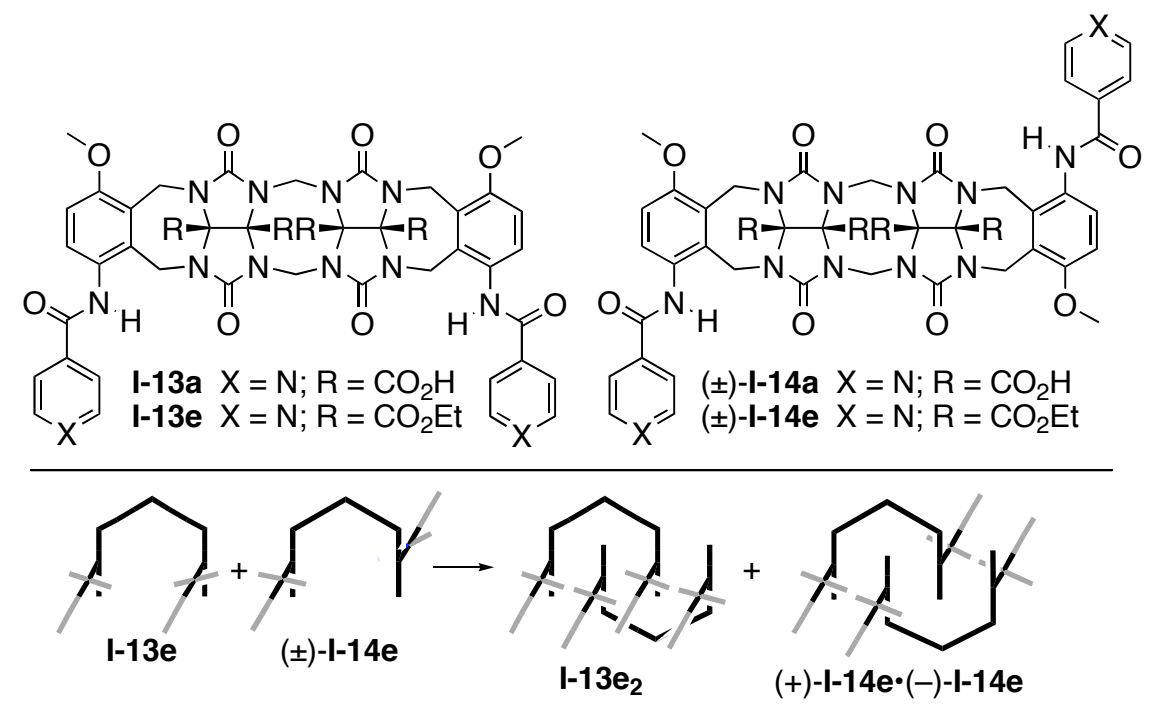

Scheme I-6. Self-sorting behavior of C-shaped molecular clips I-13e and ( \pm )-I-14e.

\subsection{Development of Complex Self-Sorting Systems.}

\subsubsection{Self-Sorting: The Exception or the Rule.}

The self-sorting systems described above were all based on the use of sets of compounds that possessed very similar structures. Accordingly, it was viewed by the community as quite exceptional behavior that these systems undergo self-sorting based on metal-ligand, $\pi-\pi$, and H-bonding interactions. In fact, despite the wide range of remarkably complex and functional systems that had been self-assembled over the years there was a perception that synthetic hosts and self-assembled systems were inferior to Natural systems in terms of strength and selectivity of binding. We decided, therefore, to create a multi-component system comprising the molecular building blocks of a series of well defined aggregates from the literature and asked whether those compounds possessed the ability to efficiently distinguish between 
self- and non-self even within a complex mixture. Would the system undergo a high fidelity self-sorting process or would cross-over heteromeric aggregation occur? For this purpose we selected $\mathbf{I}-15$ and $\mathbf{I}-16,{ }^{22-24}$ calixarene tetraurea capsule $\mathbf{I}-177_{2},{ }^{25} \mathbf{I}-18$ and I-19 which are the components of Reinhoudt's double rosette, ${ }^{26}$ Rebek's tennis ball $\mathbf{I}-\mathbf{2 0}_{2},{ }^{27}$ the components of Meijer's ureidopyrimidinone dimer $\mathbf{I}-\mathbf{2 1} \mathbf{1}_{2},{ }^{28}$ two molecular clips I-22 and I-23 (Figure I-1). We next measured the ${ }^{1} \mathrm{H}$ NMR spectrum of each aggregate separately in $\mathrm{CDCl}_{3}$ solution (Figure I-2a-h) and then measured the ${ }^{1} \mathrm{H}$ NMR spectrum of the eight component mixture (Figure I-2i). Remarkably, the ${ }^{1} \mathrm{H}$ NMR spectrum of the 9-component mixture is simply equal to the sum of the ${ }^{1} \mathrm{H}$ NMR spectra of its component aggregates. This spectroscopic earmark indicates that this 9-component mixture undergoes a high fidelity self-sorting process. We conclude that the precise pattern of H-bond donors and acceptors, the spatial distribution of those H-bonding groups, and the presence of closed networks of Hbonds are factors that favor self-sorting rather than cross-over heteromeric aggregation. ${ }^{29}$ We also studied the influence of several key variables on the selfsorting process - temperature, concentration, values of $\mathrm{K}_{\mathrm{eq}}$, and the presence of $\mathrm{H}$ bonding competitors - by a combination of simulation and experiment. Although it is clear that many systems do undergo heteromeric aggregation rather than self-sorting, this study lead us to conclude that the scope of the systems that are sufficiently selective to undergo self-sorting processes is much wider than previously appreciated. 


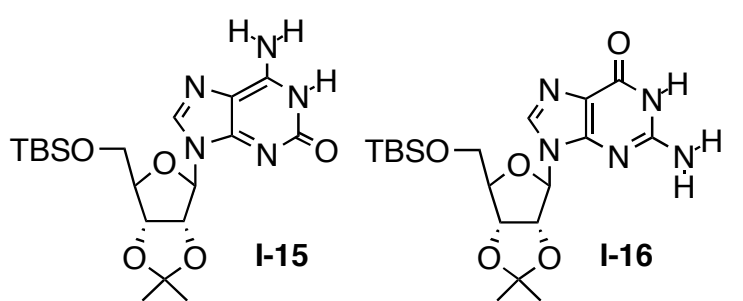
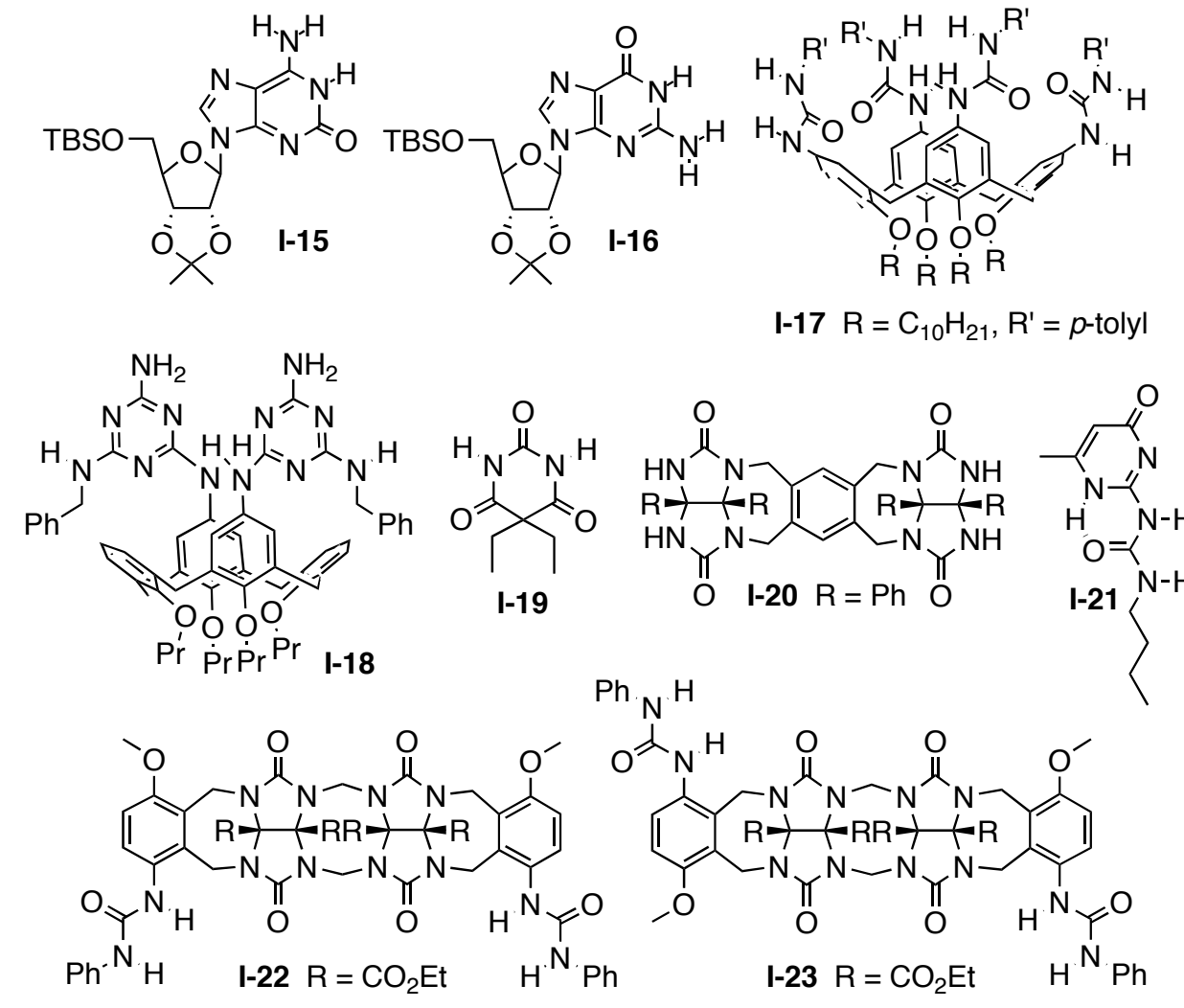

I-17 R $=\mathrm{C}_{10} \mathrm{H}_{21}, \mathrm{R}^{\prime}=$ p-tolyl

Figure I-1. Chemical structures of compounds used in H-bond directed self-sorting in $\mathrm{CDCl}_{3}$.

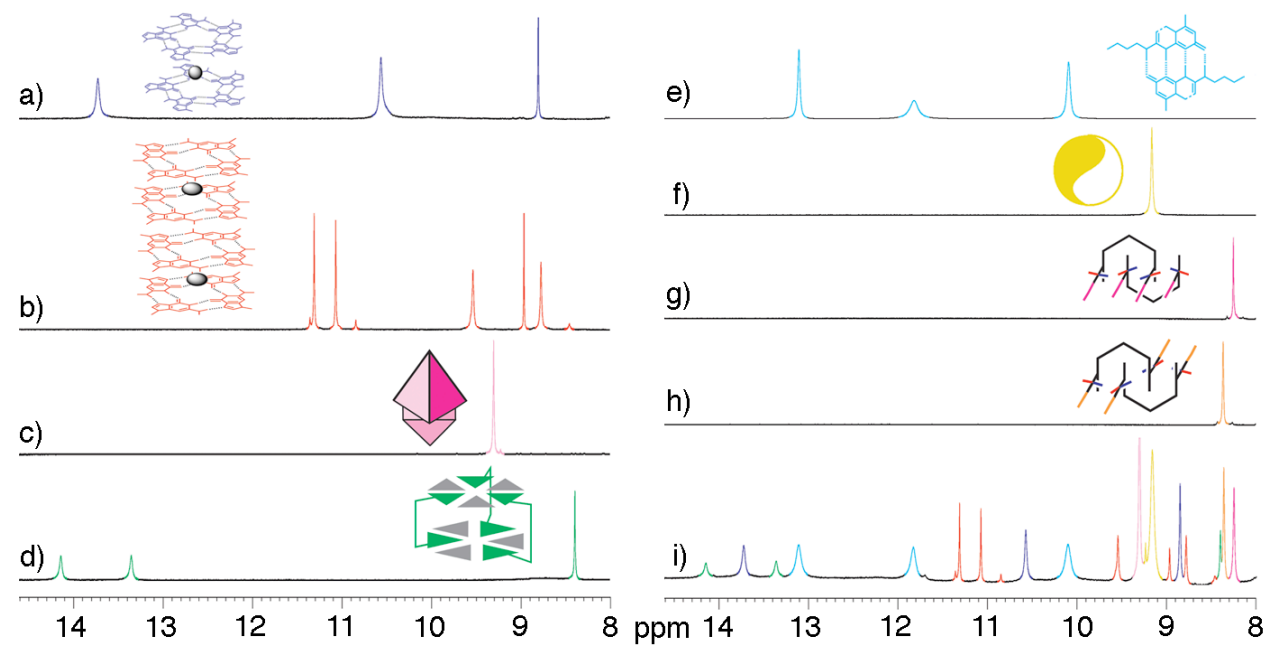

Figure I-2. Hydrogen bonding region $(8.0-14.5 \mathrm{ppm})$ of the ${ }^{1} \mathrm{H}$ NMR spectra $\left(\mathrm{H}_{2} \mathrm{O}\right.$ sat. $\left.\mathrm{CDCl}_{3}, 500 \mathrm{MHz}, 298 \mathrm{~K}\right)$ recorded for a) $\mathbf{I}_{-15} \mathbf{1 5}_{10} \cdot \mathrm{Ba}^{2+}+2$ Pic-, b) I-

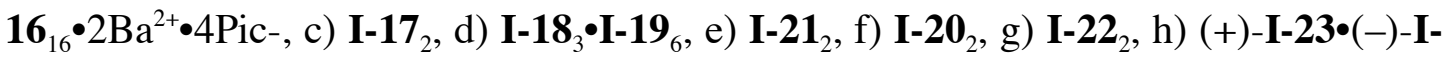


23, i) a self-sorted mixture comprising $\mathbf{I}-\mathbf{1 5}_{10} \cdot \mathrm{Ba}^{2+}+2 \mathrm{Pic}^{-}, \mathbf{I}-\mathbf{1 6}_{16} \cdot 2 \mathrm{Ba}^{2+} \bullet 4 \mathrm{Pic}^{-}, \mathbf{I}-\mathbf{1 7}_{2}$, $\mathbf{I}-18_{3} \bullet \mathbf{I}-19_{6}, \mathbf{I}-21_{2}, \mathbf{I}-\mathbf{2 0}_{2}, \mathbf{I}-22_{2}$, and (+)-I-23•(-)-I-23. The representations depict the species present in solution. The resonances are color coded to aid comparison.

\subsection{Examples of Self-Sorting Systems in Aqueous Solution.}

\subsubsection{Social Self-Sorting in Aqueous Solution.}

Although the 9-component self-sorting system described above increased the complexity of designed synthetic self-sorting systems beyond what was possible previously it suffered a number of drawbacks. First, this self-sorting process occurred in $\mathrm{CDCl}_{3}$ solution and was driven exclusively by H-bonds; in contrast Nature's selfsorting systems occur in aqueous solution driven by myriad non-covalent interactions. Second, the above system was constructed using mainly self-association processes - a subset of self-sorting processes dubbed narcissistic self-sorting by Anderson in his lovely work on oligomeric porphyrin ladders. ${ }^{30}$ Narcissistic self-sorting systems are particularly limited because strong self-association limits the number of different partners a molecule may have over its lifetime to one. In contrast, it is possible to imagine a self-sorting system composed of host-guest pairs. The advantage of such a host-guest based self-sorting system is that it is potentially environmentally responsive in that the addition of a better binding guest to the mixture would result in a change in composition of the self-sorting system. We refer to a host-guest based self-sorting system as a social self-sorting system. With these considerations in mind we selected compounds $( \pm)$-I-11, I-12 and $\mathbf{I - 2 4}-\mathbf{I}-\mathbf{3 0}$ as the components of a social 
self-sorting system in aqueous solution (Figure I-3). Compound ( \pm )-I-11 is known to undergo enantiomeric self-recognition triggered by $\mathbf{I - 1 2}$, molecular clip I-25 undergoes tight dimerization, and cryptand $\mathbf{I - 2 4}$ binds tightly to $\mathrm{K}^{+}$ion in water. The molecular containers $\beta$-CD (I-29), $\mathrm{CB}[6]$, and $\mathrm{CB}[8]$ form discrete host-guest complexes with adamantane carboxylic acid $\mathbf{I - 3 0}$, hexanediammonium ion I-26, and the charge transfer complex comprising dihydroxynaphthalene and methyl viologen (I-27•I-28), respectively. Once again we use ${ }^{1} \mathrm{H}$ NMR as our analytical technique and measure the spectra of each of the host-guest pairs separately and then measure the spectrum of the 12-component mixture. The ${ }^{1} \mathrm{H}$ NMR spectrum of the mixture is simply the sum of the ${ }^{1} \mathrm{H}$ NMR spectra of its components, which indicates this system undergoes a high fidelity social self-sorting process. ${ }^{16}$ We also studied the influence of $\mathrm{pH}$, temperature, equilibrium constant, and host:guest stoichiometry on the fidelity of self-sorting by a combination of simulation and experiment. The significance of this study lies in the demonstration that less direction interactions like ion-dipole and $\pi-\pi$ interactions and the hydrophobic effect can be used to drive self-sorting in water in much the same way more directional $\mathrm{H}$-bonds can be used in $\mathrm{CDCl}_{3}$ solution. In addition, the use of host-guest pairs as the basis of a self-sorting system (social selfsorting) offers the potential for environmental responsiveness in the form of tighter binding competitive guests. We expect this responsiveness will be one of the vehicles to achieve biomimetic function. 

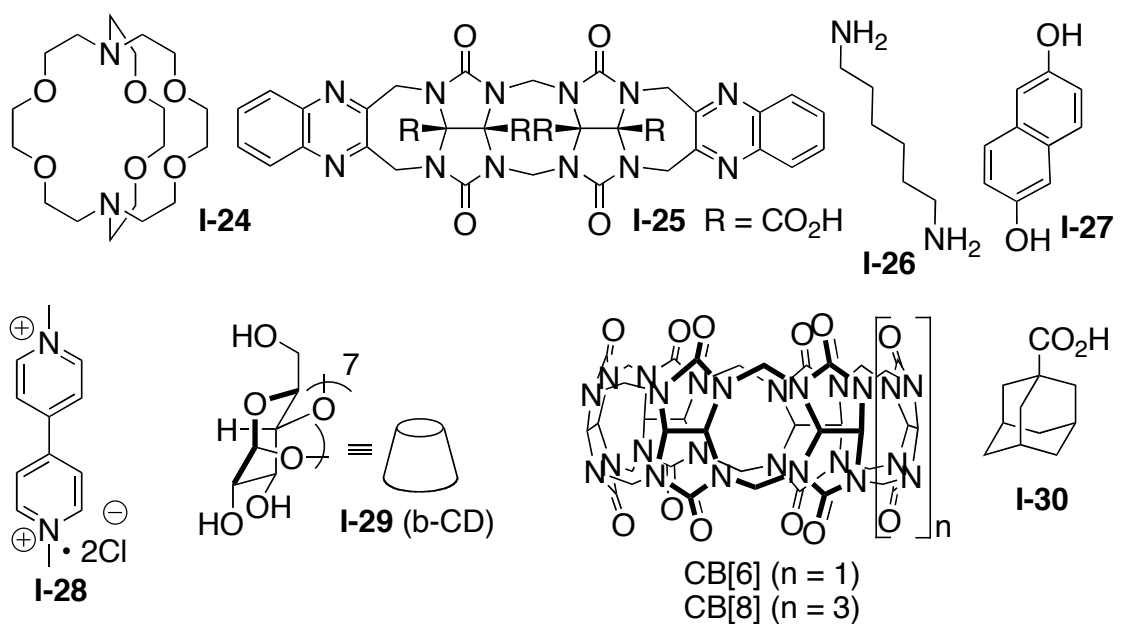

Figure I-3. Chemical structures of compounds used for social self-sorting in aqueous solution driven by ion-dipole, metal-ligand, $\pi-\pi$, and hydrophobic interactions.

\subsubsection{The $\mathrm{CB}[\mathrm{n}]$ Family of Macrocycles are Prime Components for the Construction of Self-Sorting Systems.}

In the course of preparing the 12-component social self-sorting system described above and in unpublished investigations of the complexation between $\mathrm{CB}[\mathrm{n}]$ hosts and their guests we were surprised that well defined complexes were obtained in such high fidelity processes. This suggested to us that the high binding affinity ( $\mathrm{K}_{\mathrm{a}}$ up to $10^{12} \mathrm{M}^{-1}$ ) and selectivity (up to $10^{7} \mathrm{M}^{-1}$ for $\mathbf{I - 3 1}$ between $\mathrm{CB}$ [7] and $\mathrm{CB}[8]$ ) delineated by the pioneering work of Mock for $\mathrm{CB}[6]$ would also be observed individually for the larger $\mathrm{CB}[\mathrm{n}]$ homologues $(\mathrm{CB}[7]$ and $\mathrm{CB}[8])$ and also that collectively the selectivity of $\mathrm{CB}[6], \mathrm{CB}[7]$, and $\mathrm{CB}[8]$ toward a common guest might be large. Accordingly, we measured the binding affinity of $\mathrm{CB}[6], \mathrm{CB}[7]$, and $\mathrm{CB}[8]$ toward a series of guests (I-26, I-31 - I-38) by ${ }^{1} \mathrm{H}$ NMR competition experiments. A 
selection of those values of $\mathrm{K}_{\mathrm{a}}$ are presented in Table I-1. ${ }^{31}$ Remarkably, the range of values of $\mathrm{K}_{\mathrm{a}}$ spans more then 10 orders of magnitude! Several series of complexes deserve comment (Scheme I-7). For example, consider the CB[7]・I-31 and CB[8]• I31 complexes which differ in stability by over seven orders of magnitude. Apparently, I-31 is slightly too large for $\mathrm{CB}$ [7] but has a good size and shape match with the cavity of $\mathrm{CB}[8]$. Similarly, I-37 prefers to bind to $\mathrm{CB}[7]$ over $\mathrm{CB}[6]$ due to size considerations. There are, however, situations where a guest binds tighter to the smaller $\mathrm{CB}[\mathrm{n}]$ homologue. For example, I-26 prefers to bind to $\mathrm{CB}[6]$ relative to $\mathrm{CB}[7]$ (5-fold) and $\mathbf{I - 3 8}$ prefers to bind to $\mathrm{CB}[7]$ relative to $\mathrm{CB}[8]$ (5000-fold); we attribute these preferences to a better size match between the guest and the smaller $\mathrm{CB}[\mathrm{n}]$ homologue. A final intriguing entry in Table I-1 concerns the CB[7]•I-34 and $\mathrm{CB}[8] \bullet \mathbf{I}-34$ complexes which differ in affinity by over 3000 -fold. In the CB[7]• I-34 complex only one arm of $\mathbf{I - 3 4}$ can fit inside $\mathrm{CB}[7]$; in contrast $\mathrm{CB}[8]$ induces a Ushaped turn of guest $\mathbf{I - 3 4}$ which better fills the cavity of $\mathrm{CB}[8]$. This result is significant because it suggested to us that $\mathrm{CB}[8]$ is capable of controlling the folding of abiotic oligomers in water (vide infra).

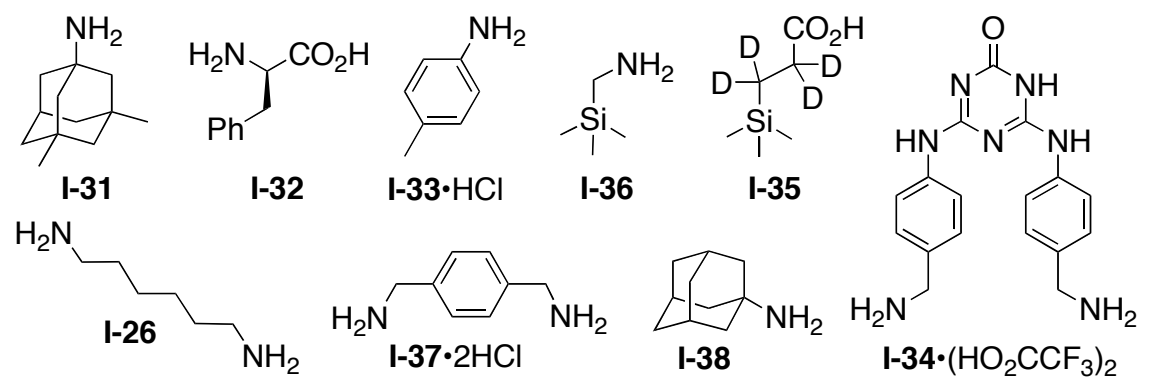


Table I-1. Values of $\mathrm{K}_{\mathrm{a}}\left(\mathrm{M}^{-1}\right)$ measured for the binding between $\mathrm{CB}[\mathrm{n}]$ hosts and guests $\left(\mathrm{D}_{2} \mathrm{O}, \mathrm{pD} 4.74,25^{\circ} \mathrm{C}\right)$.

\begin{tabular}{|l|l|l|l|}
\hline & $\mathrm{CB}[6]$ & $\mathrm{CB}[7]$ & $\mathrm{CB}[8]$ \\
\hline $\mathbf{I - 3 1}$ & - & $2.5 \times 10^{4}$ & $4.3 \times 10^{11}$ \\
\hline $\mathbf{I - 3 2}$ & $\mathrm{nb}$ & $1.5 \times 10^{5}$ & - \\
\hline $\mathbf{I - 3 3}$ & 8980 & $8.4 \times 10^{6}$ & \\
\hline $\mathbf{I - 3 4}$ & - & $1.8 \times 10^{7}$ & $5.8 \times 10^{10}$ \\
\hline $\mathbf{I - 3 5}$ & $\mathrm{nb}$ & $1.8 \times 10^{7}$ & $\mathrm{nb}$ \\
\hline $\mathbf{I - 2 6}$ & $4.5 \times 10^{8}$ & $9.0 \times 10^{7}$ & - \\
\hline $\mathbf{I - 3 6}$ & $\mathrm{nb}$ & $8.9 \times 10^{8}$ & $\mathrm{nb}$ \\
\hline $\mathbf{I - 3 7}$ & 550 & $1.8 \times 10^{9}$ & - \\
\hline $\mathbf{I - 3 8}$ & - & $4.2 \times 10^{12}$ & $8.2 \times 10^{8}$ \\
\hline
\end{tabular}

$-=$ not measured; $\mathrm{nb}=$ no binding 


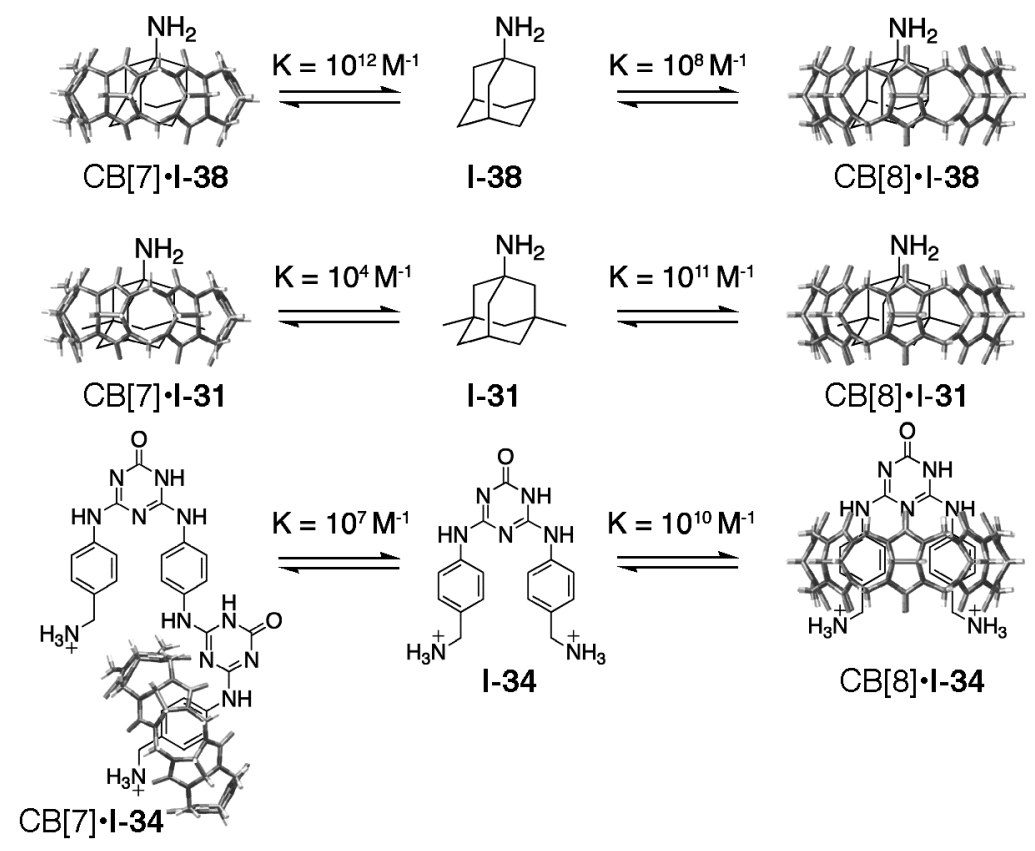

Scheme I-7. Illustration of the competition between a single guest for different sized $\mathrm{CB}[\mathrm{n}]$.

\subsubsection{Kinetic Self-Sorting.}

The sections above describe the preparation of self-sorting systems under thermodynamic control. In the design of such systems the only variables that are relevant are the concentrations of the components and the equilibrium constants of the various complexes. Living systems, of course, do not reach equilibrium until they are dead and instead use strategies to maintain metastable states and otherwise control the approach toward equilibrium. One of the common strategies employed by Nature is the use of molecular species containing multiple binding epitopes each of which serves a specific functional role. To introduce elements of temporal control into our 
self-sorting systems we decided, therefore, to synthesize and study guests containing two distinct binding epitopes (I-39 - I-42) which we refer to as two-faced guests (Scheme I-8). In designing these guests we planned to take advantage of not only the well defined thermodynamic preferences of $\mathrm{CB}[\mathrm{n}]$ toward suitable cationic guests, but also the fact that their kinetics of association and dissociation are known to span many orders of magnitude. ${ }^{32-35}$ Experimentally, we found that when a solution of $\mathrm{CB}[6]$ and $\mathrm{CB}[7]$ are mixed with a solution of $\mathbf{I - 3 9}$ and $\mathbf{I}-\mathbf{4 3}$ we observe the initial formation of the $\mathrm{CB}[6] \cdot \mathbf{I}-\mathbf{3 9}$ and $\mathrm{CB}[7] \cdot \mathbf{I}-\mathbf{4 3}$ complexes. After a period of 56 days this kinetic preference is lost and a thermodynamic self-sorting system comprising $\mathrm{CB}[6] \cdot \mathbf{I}-43$ and $\mathrm{CB}[7] \cdot \mathrm{I}-39$ were obtained $!^{36}$ Experiments involving two-faced guests with longer (e.g. I-40 and I-41) alkylammonium tails as $\mathrm{CB}[6]$ binding epitopes compromise the fidelity of the kinetic self-sorting state. By a combination of experiment and simulation we were able to determine that the major factor controlling the high fidelity of kinetic self-sorting is the fact that $\mathbf{I}-\mathbf{4 3}$ associates faster with $\mathrm{CB}[7]$ than I-39 does despite the fact that the $\mathrm{CB}[7] \cdot \mathbf{I}-39$ complex is thermodynamically more stable than $\mathrm{CB}[7] \cdot \mathbf{I}-\mathbf{4 3}$. This situation probably arises because the ureidyl $\mathrm{C}=\mathrm{O}$ lined portals of $\mathrm{CB}[\mathrm{n}]$ are narrower than the cavity which they guard which may result in large barriers to association and dissociation for guests which optimally fill the $\mathrm{CB}[\mathrm{n}]$ cavity. The influences of metal cations (identity and concentration) on the fidelity of the kinetic and thermodynamic selfsorting were also investigated. One of the most interesting aspects of this investigation was the post facto deconstruction of the system which revealed that the $\mathrm{CB}[6] \cdot \mathbf{I}-\mathbf{4 3}$ complex is governed by remarkably slow kinetics of association $\left(\mathrm{k}_{\mathrm{on}}=\right.$ 
$\left.0.0012 \mathrm{M}^{-1} \mathrm{~s}^{-1}\right)$ and dissociation $\left(\mathrm{k}_{\mathrm{off}}=8.5 \times 10^{-10} \mathrm{~s}^{-1}\right) .{ }^{36}$ This dissociation rate constant - which is approximately 100 -fold slower than biotin•avidin - corresponds to a half-life of 26 years at room temperature! Because the outcome of self-sorting experiments simultaneously probes the entire matrix of kinetic constants and thermodynamic parameters (Scheme I-8) these simple experiments are capable of revealing truly anomalous host-guest binding experiments rapidly. As uncovering and understanding such anomalous binding events are one of the key subjects for supramolecular chemists we believe that self-sorting systems have much to offer to the community.

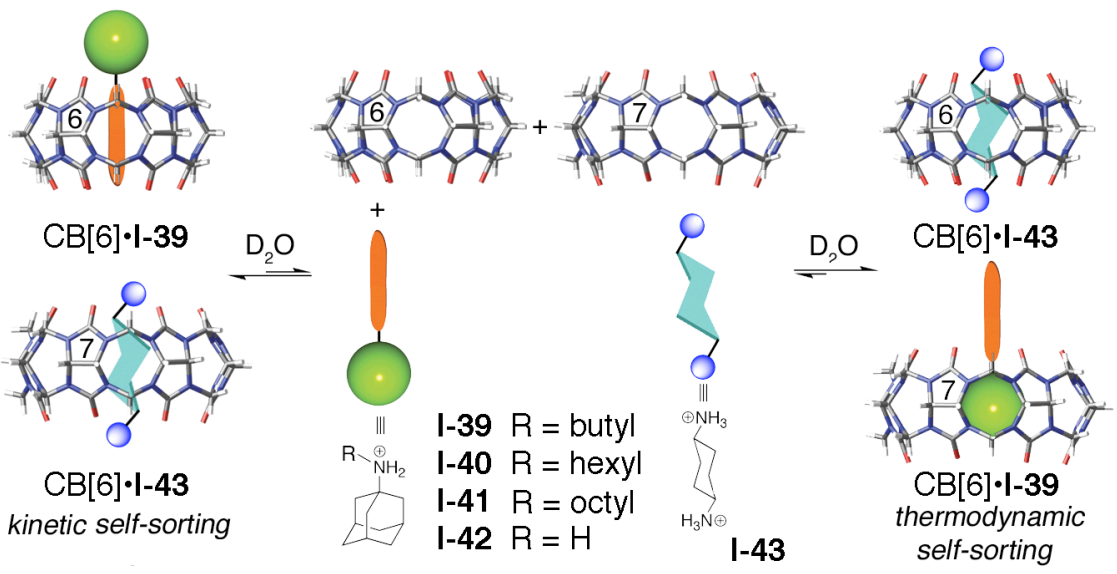

Scheme I-8. Kinetic and thermodynamic self-sorting based on guests with two binding epitopes.

\subsubsection{Self-Sorting Processes Control the Folding, Forced Unfolding, and Refolding of an Abiotic Oligomer in Water.}

Based on the high association constants observed for $\mathrm{CB}[7] \bullet \mathrm{I}-34$ and $\mathrm{CB}[8] \bullet$

I-34, the high selectivity observed between these two complexes and the interesting 
folding induced by $\mathrm{CB}[8]$ we wondered whether longer oligomers might be induced to exhibit well defined conformational preferences in the presence of the various $\mathrm{CB}[\mathrm{n}](\mathrm{n}=7,8,10)$. For this purpose, we designed and synthesized a number of arylene-triazene oligomers exemplified by compound I-44. Compound I-44 contains four (guanidine-like) triazene-N bonds (highlighted with curved arrows) which may adopt two different rotamers (Figure I-4). ${ }^{37}$ Of the $2^{4}$ (16) possible conformations, 10 are unique. Through a combination of NMR spectroscopy and x-ray crystallography we were able to determine that the complexation of $\mathbf{I - 4 4}$ with $\mathrm{CB}$ [7], $\mathrm{CB}[8]$, or $\mathrm{CB}[10]$ leads to the formation of well defined $\mathrm{CB}[7] \bullet(\mathrm{a}, \mathrm{s}, \mathrm{s}, \mathrm{a})-\mathbf{I}-\mathbf{4 4} \cdot \mathrm{CB}[7]$, $\mathrm{CB}[8] \bullet(\mathrm{a}, \mathrm{a}, \mathrm{a}, \mathrm{s})-\mathbf{I}-\mathbf{4 4}$, and $\mathrm{CB}[10] \bullet(\mathrm{a}, \mathrm{a}, \mathrm{a}, \mathrm{a})-\mathbf{I}-\mathbf{4 4}$ complexes (Scheme I-9). Perhaps most interesting, however, is how the high affinity and selectivity of $\mathrm{CB}[\mathrm{n}]$ complexes can be used as a thermodynamic driving force to sequentially induce the folding, unfolding, and refolding of abiotic oligomer I-44 into four different conformations. We first add $\mathrm{CB}[8]$ to a solution of $\mathbf{I - 4 4}$ which populates the full 10component conformational ensemble open to I-44 which induces the folding of I-44 to yield the $\mathrm{CB}[8] \bullet(\mathrm{a}, \mathrm{a}, \mathrm{a}, \mathrm{s})-\mathbf{I}-\mathbf{- 4 4}$ conformation by maximization of ion-dipole and $\mathrm{H}$ bonding to the ureidyl $\mathrm{C}=\mathrm{O}$ portals of $\mathrm{CB}[8]$ and the hydrophobic effect. By addition of I-31 (1 equiv.) and CB[7] (2 equiv.) it is possible to eject $\mathbf{I - 4 4}$ from the cavity of $\mathrm{CB}[8]$ under the formation of $\mathrm{CB}[8] \cdot \mathbf{I}-\mathbf{3 1}$ and then refold $\mathbf{I - 4 4}$ with the help of $\mathrm{CB}[7]$ to yield $\mathrm{CB}[7] \bullet(\mathrm{a}, \mathrm{s}, \mathrm{s}, \mathrm{a})-\mathbf{I}-\mathbf{4 4} \cdot \mathrm{CB}[7]$. In this process the high affinity of $\mathbf{I - 3 1}$ for $\mathrm{CB}[8]\left(\mathrm{K}_{\mathrm{a}}=4.3 \times 10^{11} \mathrm{M}^{-1}\right)$ ensures the transformation into a high-fidelity self-sorting state. If this transformation is conducted stepwise by the addition of $\mathrm{CB}$ [7] first, it is possible to spectroscopically observe the intermediacy of $\mathrm{CB}[8] \bullet(a, a, s, a)-$ I- 
44• $\mathrm{CB}[7]$. Remarkably, control experiments show that the binding of $\mathrm{CB}[7]$ to the tail of guest I-44 catalyzes its dissociation from the cavity of CB[8]. Finally, addition of I-38 (2 equiv.) to $\mathrm{CB}[7] \cdot(\mathrm{a}, \mathrm{s}, \mathrm{s}, \mathrm{a})-\mathbf{I}-\mathbf{4 4} \cdot \mathrm{CB}[7]$ results in the formation of $\mathrm{CB}[7] \cdot \mathbf{I}-\mathbf{3 8}$ $\left(\mathrm{K}_{\mathrm{a}}=4.2 \times 10^{12} \mathrm{M}^{-1}\right)$, ejection of $\mathbf{I}-\mathbf{4 4}$ into free solution where it forms its equilibrium mixture of conformers. Subsequently, I-44 ejects $\mathrm{CB}[5]$ from the $\mathrm{CB}[10] \cdot \mathrm{CB}[5]$ complex to yield a self-sorted state comprising $\mathrm{CB}[10] \bullet(a, a, a, a)-\mathbf{I}-44$, free $\mathrm{CB}[5]$, $\mathrm{CB}[8] \bullet \mathbf{I}-31$, and $\mathrm{CB}[7] \cdot \mathbf{I}-\mathbf{3 8}$. This study illustrates how it is possible to use high affinity and highly selective receptors like $\mathrm{CB}[\mathrm{n}]$ and their associated large values of $\Delta \mathrm{G}$ of binding to mimic an important biological event - namely the folding, unfolding, and refolding of an oligomeric compound in water.
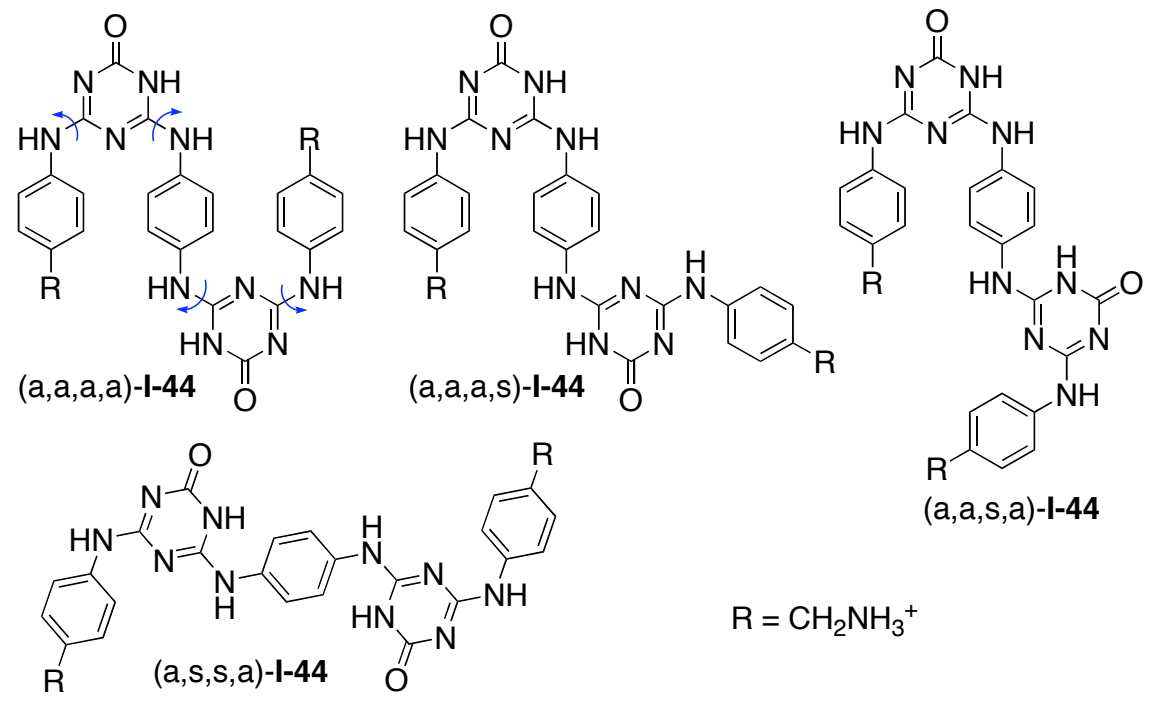

$(\mathrm{a}, \mathrm{a}, \mathrm{S}, \mathrm{a})-\mathrm{I}-44$

Figure I-4. Chemical structures of four of the ten conformational isomers of I-44. Arrows highlight the four two-fold rotors. 


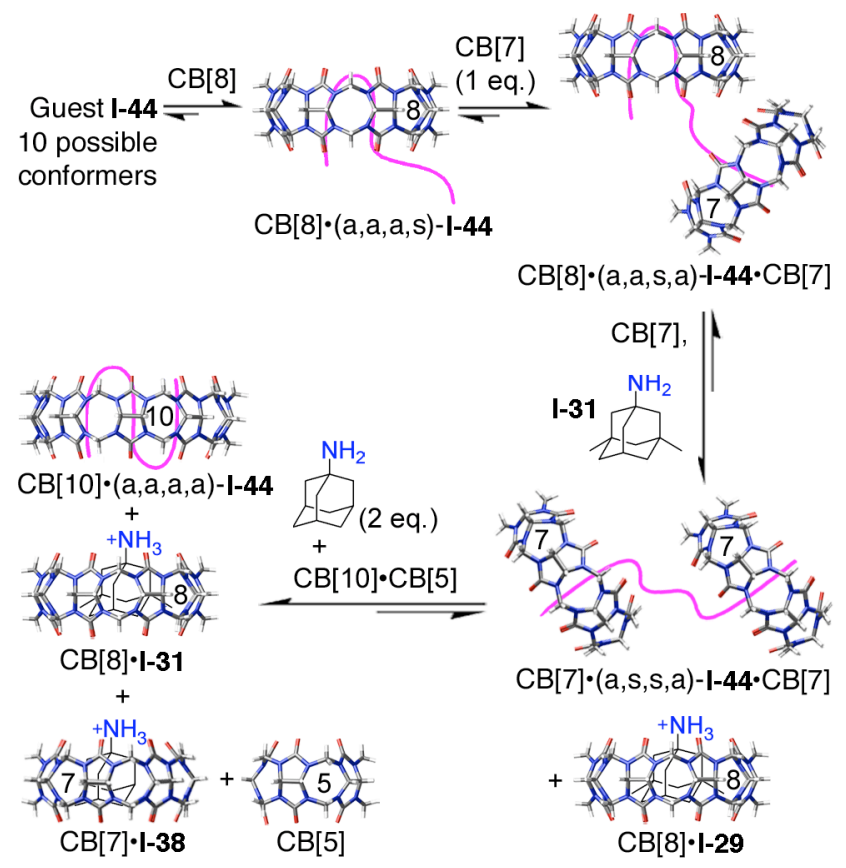

Scheme I-9. The sequential addition of various $\mathrm{CB}[\mathrm{n}]$ and guests to I-44 induces folding, forced unfolding, and refolding of $\mathbf{I - 4 4}$ into four different conformations.

\subsection{Selected Examples of Self-Sorting Systems from the Literature.}

A number of laboratories have been pursuing the development of self-sorting systems either explicitly or implicitly. In this section we present a selection of those systems based on a variety of non-covalent interactions.

\subsubsection{Self-sorting Systems Based on Porphyrin Coordination Chemistry.}

The earliest example of a porphyrin derived self-sorting system comes from the Anderson group. ${ }^{30}$ The Anderson group studied the formation of porphyrin ladders from butadiyne linked linear porphyrin dimer though hexamer in combination 
with diazabicyclooctane. They observed a variety of interesting behaviour including positive cooperativity, two-state assembly, large Hill coefficients, and narcissistic self-sorting. More recently, the Osuka group has studied the assembly and self sorting of meso-meso linked porphyrin dimers bearing pyridyl substituents. ${ }^{38-40} \mathrm{~A}$ beautiful example arises from the assembly of meso-cinchomerimide appended diporphyrin I-45. Compound I-45 contains three axes of chirality - the meso-meso porphyrin link and the two cinchomerimide-porphyrin linkages - which leads to six different atropisomers enumerated in Scheme I-10. These six atropisomers exist as three pairs of enantiomers, namely the in-in, in-out, and out-out atropisomers that differ in the angle between the two coordinating $\mathrm{N}$-atoms of the cinchomerimide groups. Remarkably, the atropisomer with the smallest angle $\mathbf{I}-\mathbf{4 5}_{\text {in-in }}$ leads to a triangular trimeric porphyrin box $\left(\mathbf{I}-\mathbf{4 5} \mathbf{5}_{\text {in-in }}\right)_{3}$ whereas the atropisomers with larger angles $\mathbf{I}-\mathbf{4 5}_{\text {in-out }}$ and $\mathbf{I}-\mathbf{4 5}_{\text {out-out }}$ lead to tetrameric and pentameric porphyrin boxes $(\mathbf{I}-\mathbf{4 5}$ in$\left.{ }_{\text {out }}\right)_{4}$ and $\left(\mathbf{I}-\mathbf{- 5}_{\text {out-out }}\right)_{5}$, respectively. Even more remarkable is that this self-sorting assembly process occurs in a homochiral fashion whereby all molecules of I-45 in a given assembly are of a single handedness (e.g. $\left(R-\mathbf{I}-\mathbf{4 5} \mathbf{5}_{\text {in-in }}\right)_{3}$ and $\left.\left(S-\mathbf{I}-\mathbf{4 5} \mathbf{5}_{\text {in-in }}\right)_{3}\right)$. This result nicely demonstrates that subtle conformational and stereochemical information can be efficiently translated from simple building blocks to far more complex assemblies. 

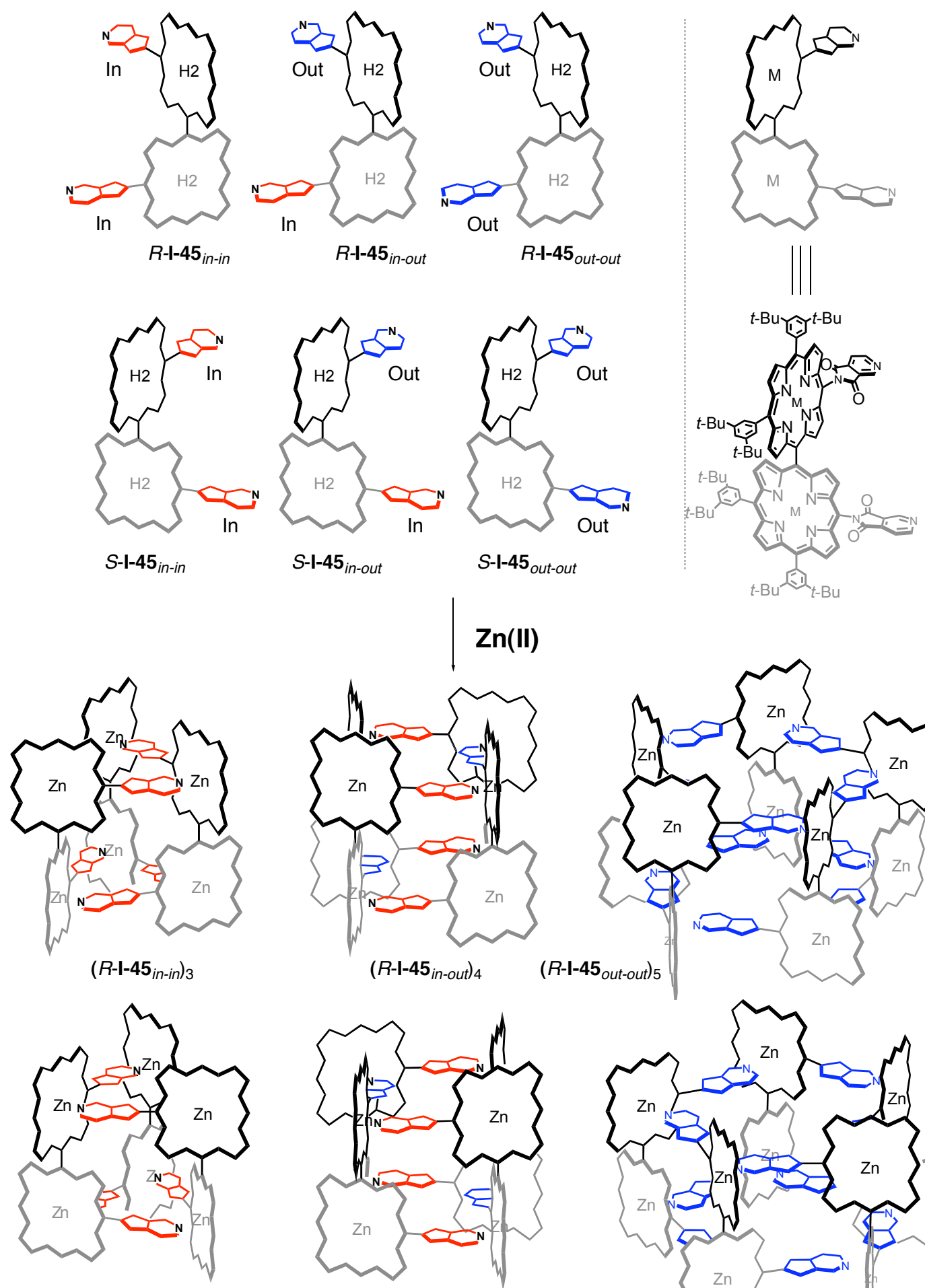

$\left(S-1-45_{i n-i n}\right)_{3}$

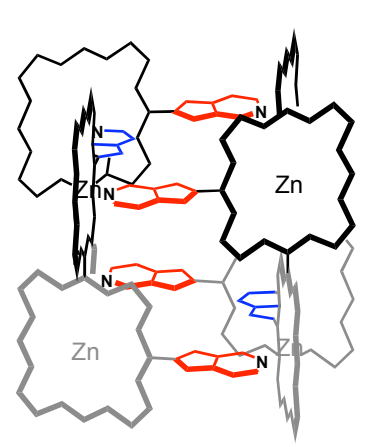

$\left(S-1-45_{\text {in-out }}\right)_{4}$
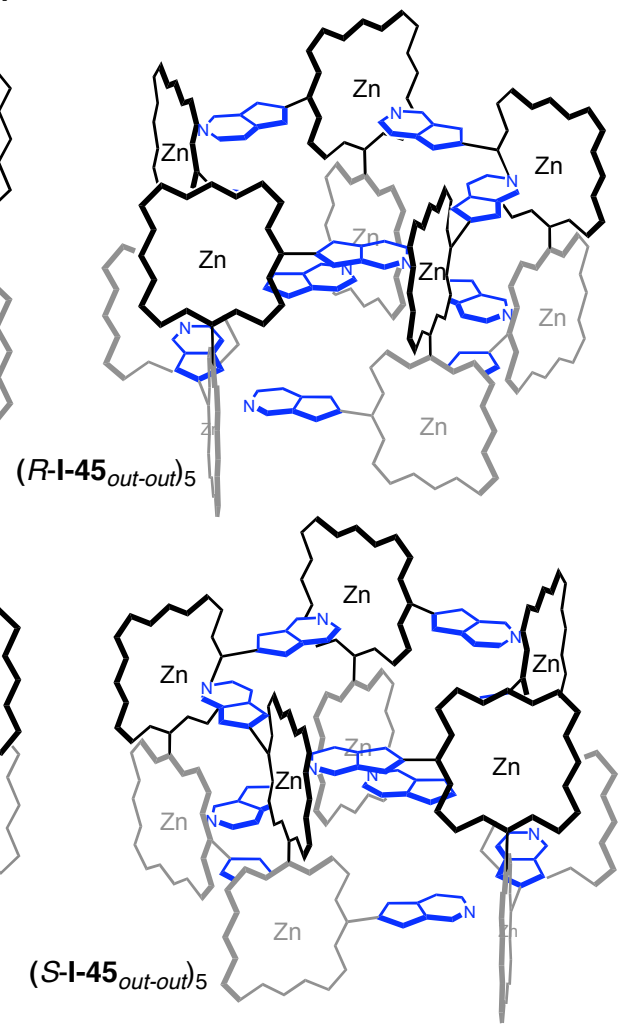

Scheme I-10. Homochiral self-sorting assembly of meso-linked porphyrin I-45. 


\subsubsection{Self-sorting Based on Hydrogen Bonding Interactions.}

The Zimmerman group has studied the self-assembly of H-bonding modules derivatized with dendrons of different generations in non-polar solvents by $\mathrm{H}$ bonding interactions. ${ }^{41,42}$ For example, Zimmerman and co-workers find that I-46 G1 which contains DAA and DDA H-bonding faces with $60^{\circ}$ relative orientation undergoes self-assembly to yield hexamer $\left(\mathbf{I}-46\right.$ - G1) ${ }_{6}$ (Scheme I-11) as evidence by size exclusion chromatography (SEC). Similarly, dimeric compound I-47 - G3 with its self-complementary DDAA faces forms an exceptionally stable cyclic hexamer (I47 - G3) $)_{6}$ by H-bonding interactions. Very interestingly, a mixture of I-46 - G1 and I-47 - G3 undergoes a high-fidelity self-sorting process to yield the individual hexamers $(\mathbf{I}-46 \text { - G1 })_{6}$ and $(\mathbf{I}-47 \text { - G3 })_{6}$ driven by the different patterns of H-bonds and relative orientation of their faces; SEC monitoring of this mixture over the course of 53 days does not reveal any evidence of cross-over heteromeric aggregation. Quite interestingly, when a mixture of I-46 - G1 and I-46 - G3 - which contain identical H-bonding information, but different sized dendritic wedges on their periphery - is prepared a high fidelity social self-sorting process is observed under the the formation of the mixed aggregate $(\mathbf{I}-\mathbf{4 6}-\mathbf{G 1})_{3} \bullet(\mathbf{I}-\mathbf{4 6}-\mathbf{G 3})_{3}$ wherein small dendritic wedges are adjacent to large dendritic wedges and vice versa. This process proceeds via the kinetic intermediacy of a dynamic combinatorial library of the 11 distinct hexameric aggregates containing all combinations of one - five equivalents of I-46 - G1 and I46 - G3 (Scheme I-12). 

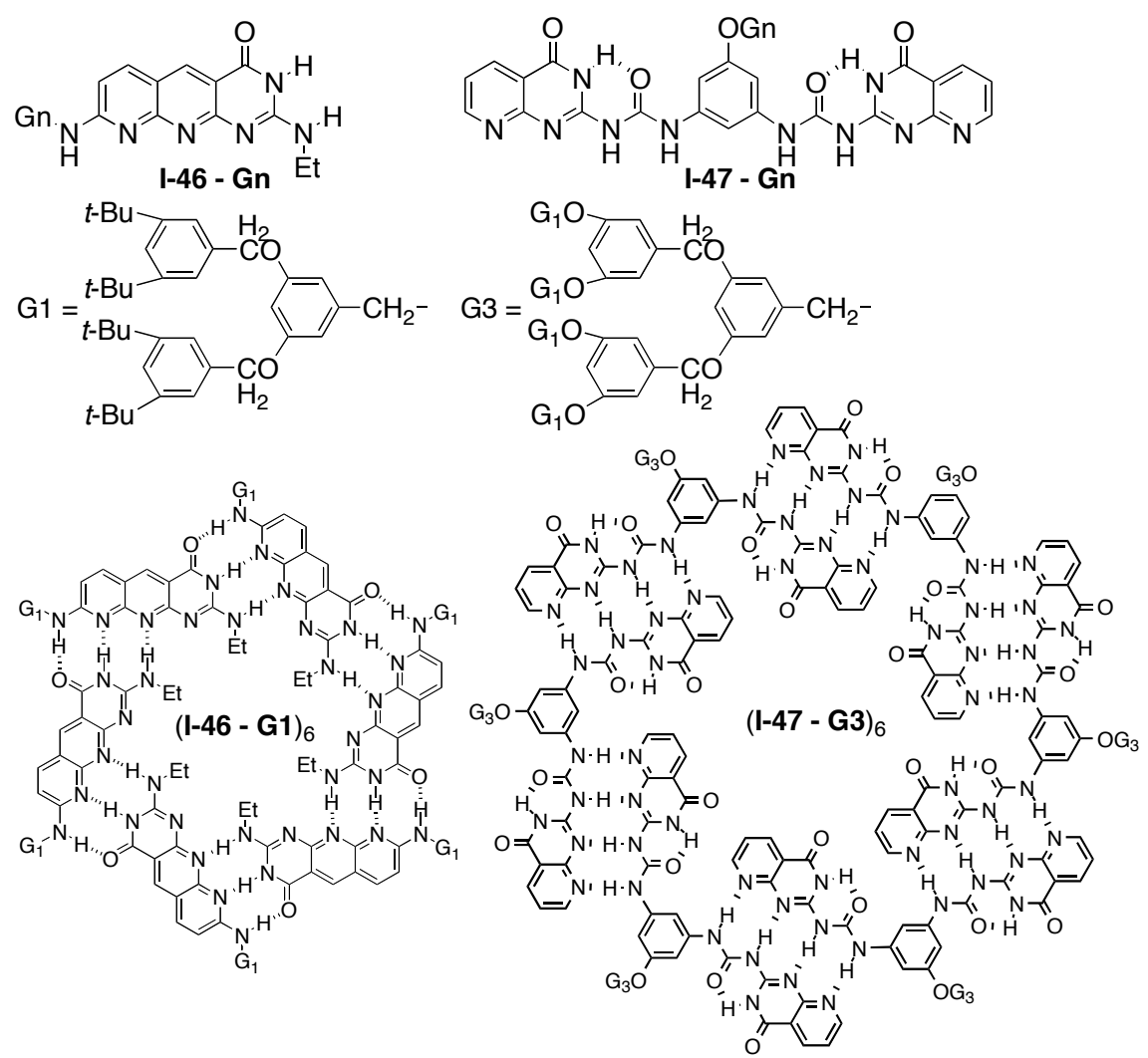

Scheme I-11. Hexameric assemblies based on DDA-AAD H-bonding motif.

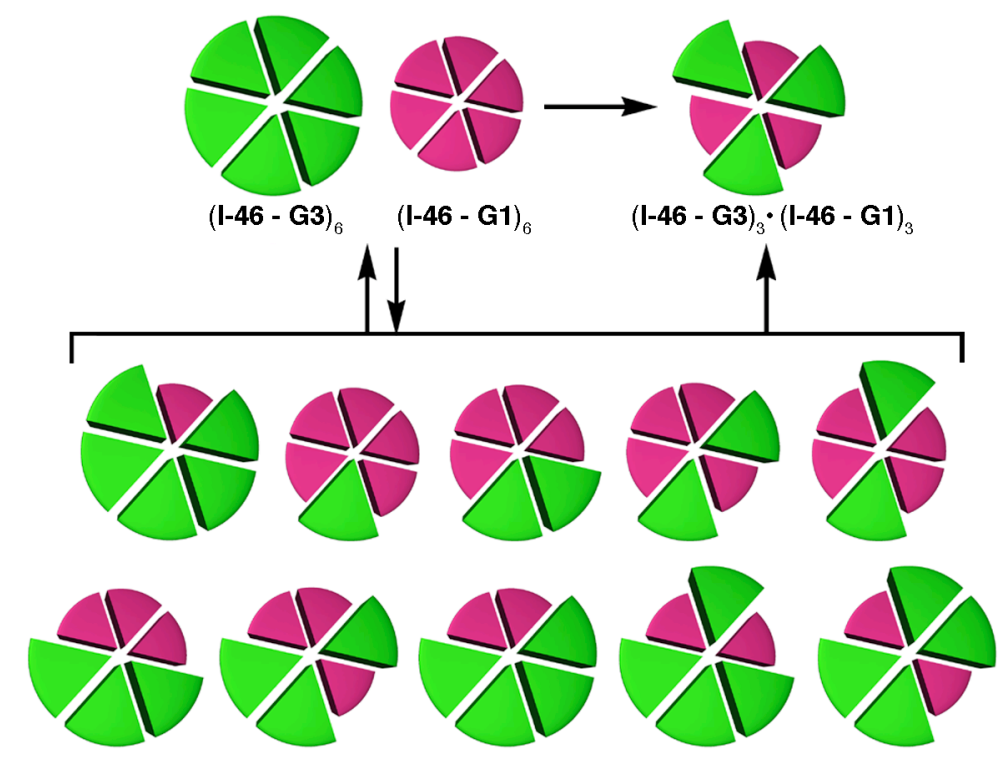

Scheme I-12. Mixed aggregates formed from mixing of (I-46 - G1) 6 and (I-46 G3) $)_{6}$ 


\subsubsection{Self-Sorting Coiled Coil.}

The assembly of $\alpha$-helical coiled coils from amphiphilic peptides depends subtly on the sequence of the oligomer with a variety of important biologically consequences. ${ }^{43}$ Consequently, the control over the assembly of such (designed and/or non-natural) peptides has been the subject of numerous investigations. Here we focus on the work from the laboratories of Kumar and Kennan that involve selfsorting processes. In 2001, Kumar's group reported the synthesis of two amphiphilic peptides $(\mathbf{H}$ and $\mathbf{F})$ - one containing a hydrophobic face composed of leucine residues (H) and one containing hexafluoroleucine residues $(\mathbf{F})$ - terminated in cysteine residues. $^{44,45}$ When the mixture of the fluorophobic and hydrophobic peptides were allowed to form disulfides under oxidizing conditions in water only the $\mathbf{H H}$ and $\mathbf{F F}$ peptides were formed (Scheme I-13). This high fidelity self-sorting process is driven by solvophobic effect. Schnarr and Kennan reported a very interesting system in which six different peptides spontaneously assemble into three different 1:1:1 heterotrimers in a self-sorting process (Scheme I-14). ${ }^{46}$ To promote the 1:1:1 heterotrimer specificity Schnarr and Kennan utilized a steric matching approach ${ }^{47}$ based on the size of hydrophobic side chains (alanine versus cyclohexylalanine) at positions 9,16 , and 23 of the peptide. Only one cyclohexylalanine may reside at a given level of the interface of the 1:1:1 heterotrimer. As a secondary recognition element the charge (e.g. lysine versus glutamic acid) at the residues adjacent (positions e and $\mathrm{g}$ ) to the hydrophobic interface were employed. Remarkably, a 2:2:2:1:1:1 mixture of $\mathbf{T}_{9} \mathbf{K}, \mathbf{T}_{16} \mathbf{K}, \mathbf{T}_{23} \mathbf{K}, \mathbf{T}_{\mathbf{9}} \mathbf{E}, \mathbf{T}_{\mathbf{1 6}} \mathbf{E}$, and $\mathbf{T}_{23} \mathbf{E}$ forms a mixture of the three heterotrimers $\mathbf{T}_{9} \mathbf{E} \cdot \mathbf{T}_{16} \mathbf{K} \cdot \mathbf{T}_{23} \mathbf{K}, \mathbf{T}_{9} \mathbf{K} \cdot \mathbf{T}_{16} \mathbf{K} \cdot \mathbf{T}_{23} \mathbf{K}$, and $\mathbf{T}_{9} \mathbf{K} \cdot \mathbf{T}_{16} \mathbf{E} \cdot \mathbf{T}_{23} \mathbf{K}$ in a high 
fidelity self-sorting process (Scheme I-14). These same authors have also shown how addition of specific peptides to such a mixture can be used to trigger a change in the composition $^{48-50}$ in a way similar to that reported above for the folding of non-natural oligomers inside $\mathrm{CB}[\mathrm{n}]$ molecular containers.

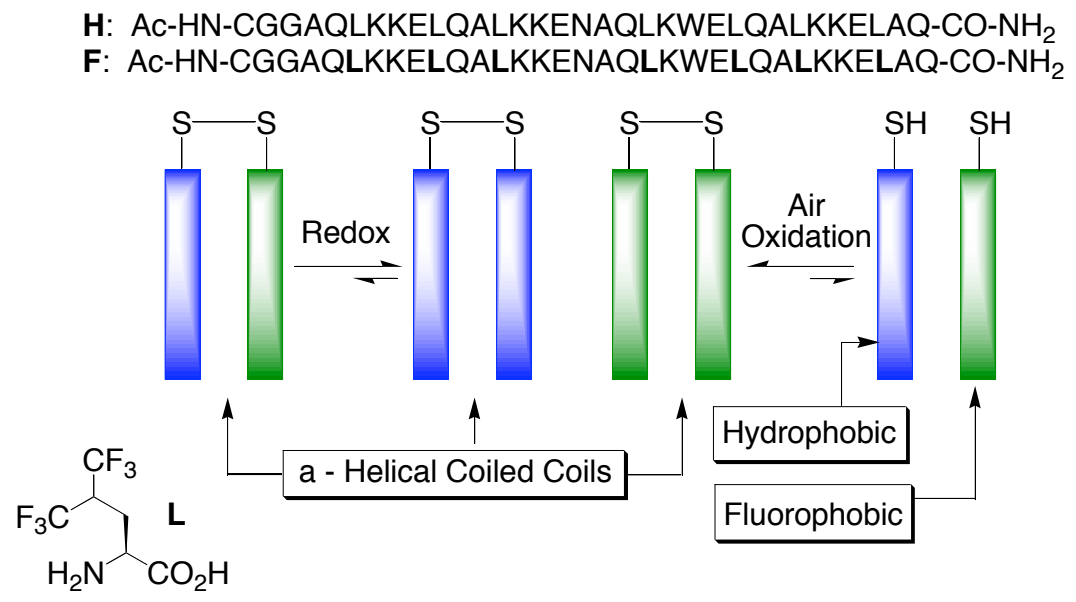

Scheme I-13. Homodimer formation by fluorous and hydrocarbon core peptides. 

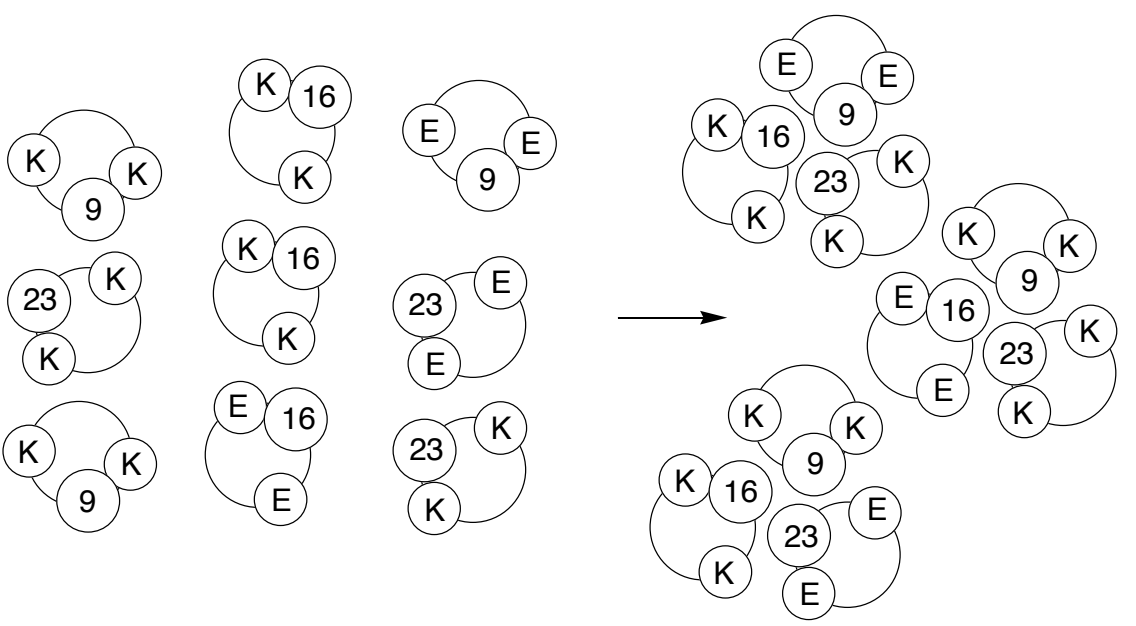

$\mathrm{T}_{9} \mathrm{E}:$ AcNH-EMKQLEKEXEELESEAQQLEKEAAQLEKEVG-Am

$\mathrm{T}_{9} \mathrm{~K}:$ AcNH-KMKQLKKKXEELKSKAQQLKKKAAQLKKKVG-Am

$\mathrm{T}_{16} \mathrm{E}:$ AcNH-EMKQLEKEAEELESEXQQLEKEAAQLEKEVG-Am

$\mathrm{T}_{16} \mathrm{E}:$ AcNH-KMKQLKKKAEELKSKXQQLKKKAAQLKKKVG-Am

$\mathrm{T}_{23} \mathrm{E}$ : AcNH-EMKQLEKEAEELESEAQQLEKEXAQLEKEVG-Am

$\mathrm{T}_{23} \mathrm{E}:$ AcNH-KMKQLKKKAEELKSKAQQLKKKXAQLKKKVG-Am

Scheme I-14. Self-sorting within amphiphilic peptides driven by steric matching and electrostatic effects.

1.6.8 Construction, Substitution, and Sorting of Metallo-organic Structures via Subcomponent Self-Assembly.

The group of Jonathan Nitschke has been particularly active recently in the development of self-sorting systems based on metal-ligand interactions. Here we describe one recent contribution to the area $;{ }^{51}$ the reader is referred to a recent review for more comprehensive information. ${ }^{52}$ Sarma and Nitschke report an extremely well-defined system comprising anilines I-48 and I-49, aldehydes I-50 - I-52, and $\mathrm{Cu}^{+}$for which they have elucidated the rules that govern the nature and concentration of all products formed (Scheme I-15). In DMSO/MeCN solution an equilibrium is 
established with the eight possible imines (I-53 - I-60) indicating no innate preference exists for a specific imine. Remarkably, when $\mathrm{Cu}^{+}$was added to the system, a self-sorting process occurred that resulted in the formation of equimolar amounts of four discrete products (I-61 - I-64). This system is so well-defined that "more complex mixtures, having arbitrary product ratios are predicted to be readily accessible $\ldots$ by simply mixing together subcomponents in the ratio in which they are found in the desired collection of product structures."
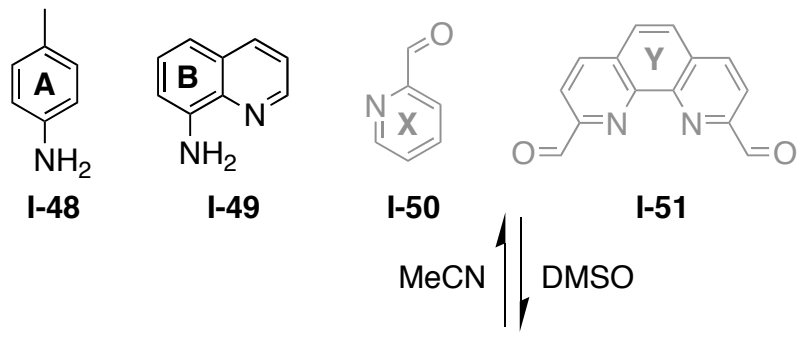

$1-51$
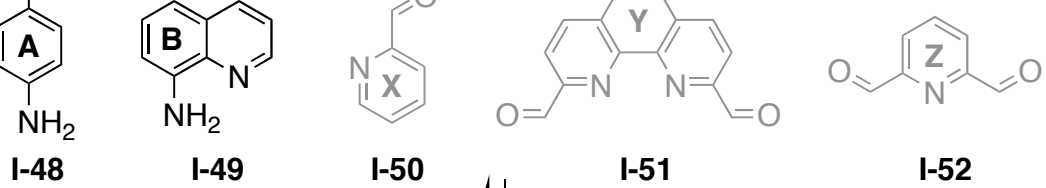

I-49

I-52
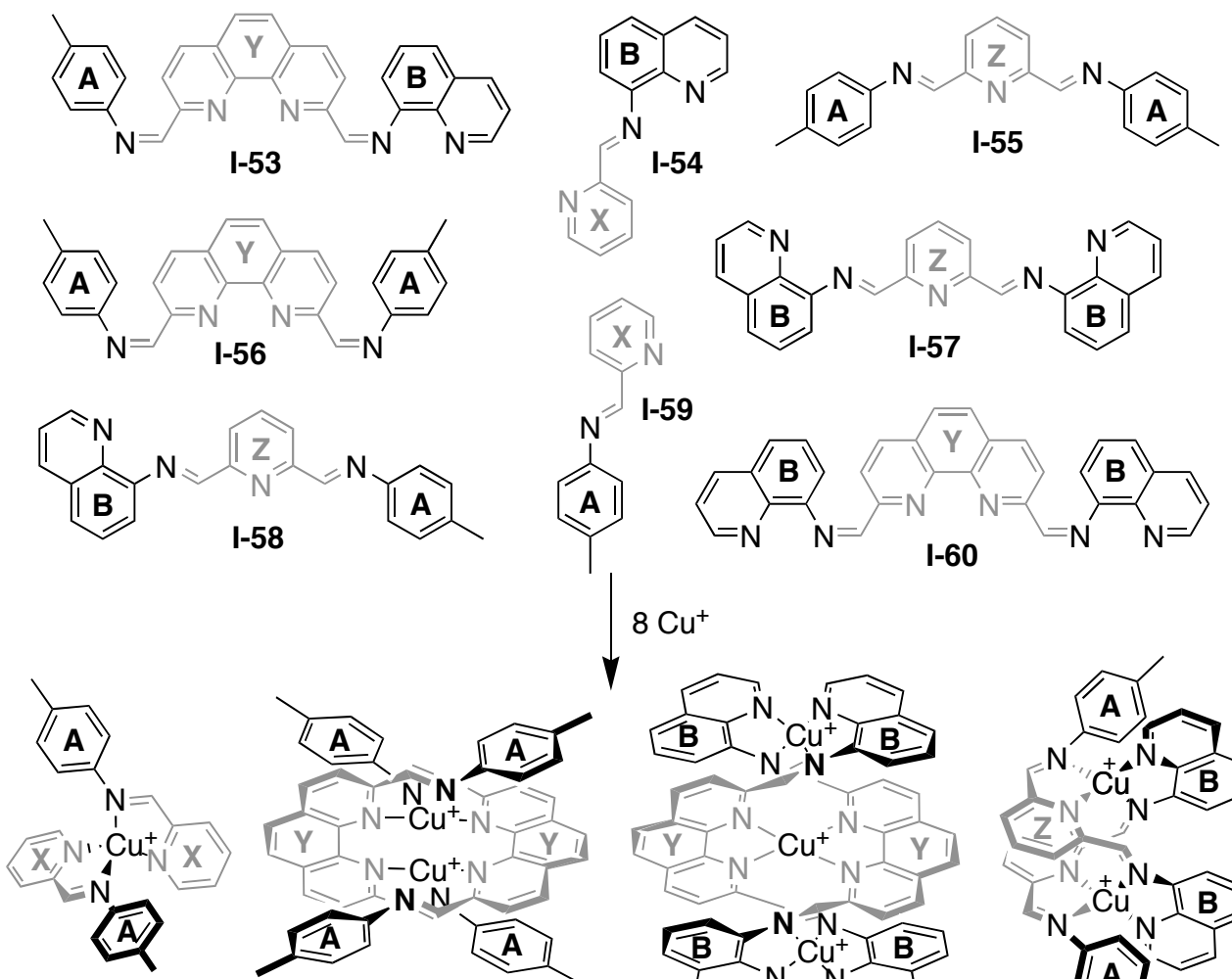

$1-61$

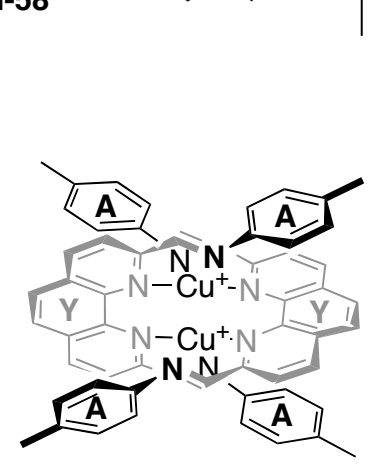

$1-62$

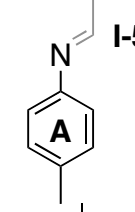

$-59$
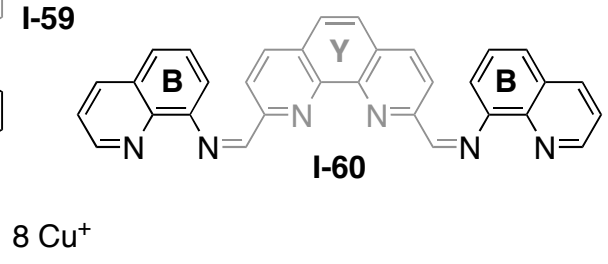

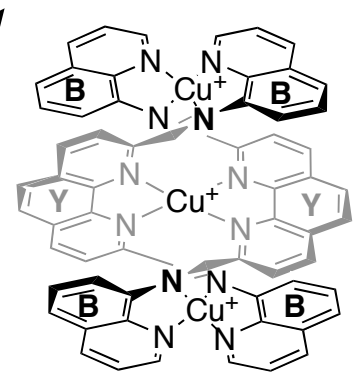

I-63

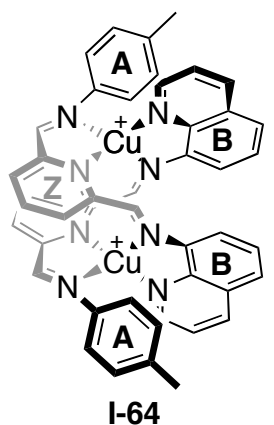

Scheme I-15. Formation of a dynamic imine library and $\mathrm{Cu}^{+}$templated self-sorting. 


\subsection{Application of Self-Sorting in Material Science and Nanotechnology.}

The ability of certain systems to undergo high-fidelity self-sorting processes allows the precise positioning of molecules from within a complex mixture. Accordingly, there are a number of applications in materials science and nanotechnology that are enabled by self-sorting processes. This section discusses several examples of representative applications.

1.8.1 Self-Assembly of Polymers and Nanoparticles on Patterned Surfaces.

Rotello and co-workers report a very interesting example of self-sorting on a patterned surface (Scheme I-16). ${ }^{53}$ For this purpose, a Si wafer was spin coated with a cationic polymer (PVMP) which was then photo-crosslinked. Patterning was performed by spin-coating a layer of Thy-PS followed by treating with UV light through a photoresist mask. The resulting square patterned surface contains two recognition regions - one based on PVMP electrostatic interactions and one based on the H-bonding ADA pattern of the Thy-PS polymer. Interestingly, applying solutions of either anionic nanoparticles COO-NP or diaminopyridine derived DAD H-bonding polymer DAP-PS results in the selective derivatization of the complementary region of the surface. The simultaneous application of both DAP-PS and COO-NP results in the derivatization of both regions in a self-sorting process. The work provides a step toward the rapid, multicomponent, three-dimensional fabrication of complex functional materials. 


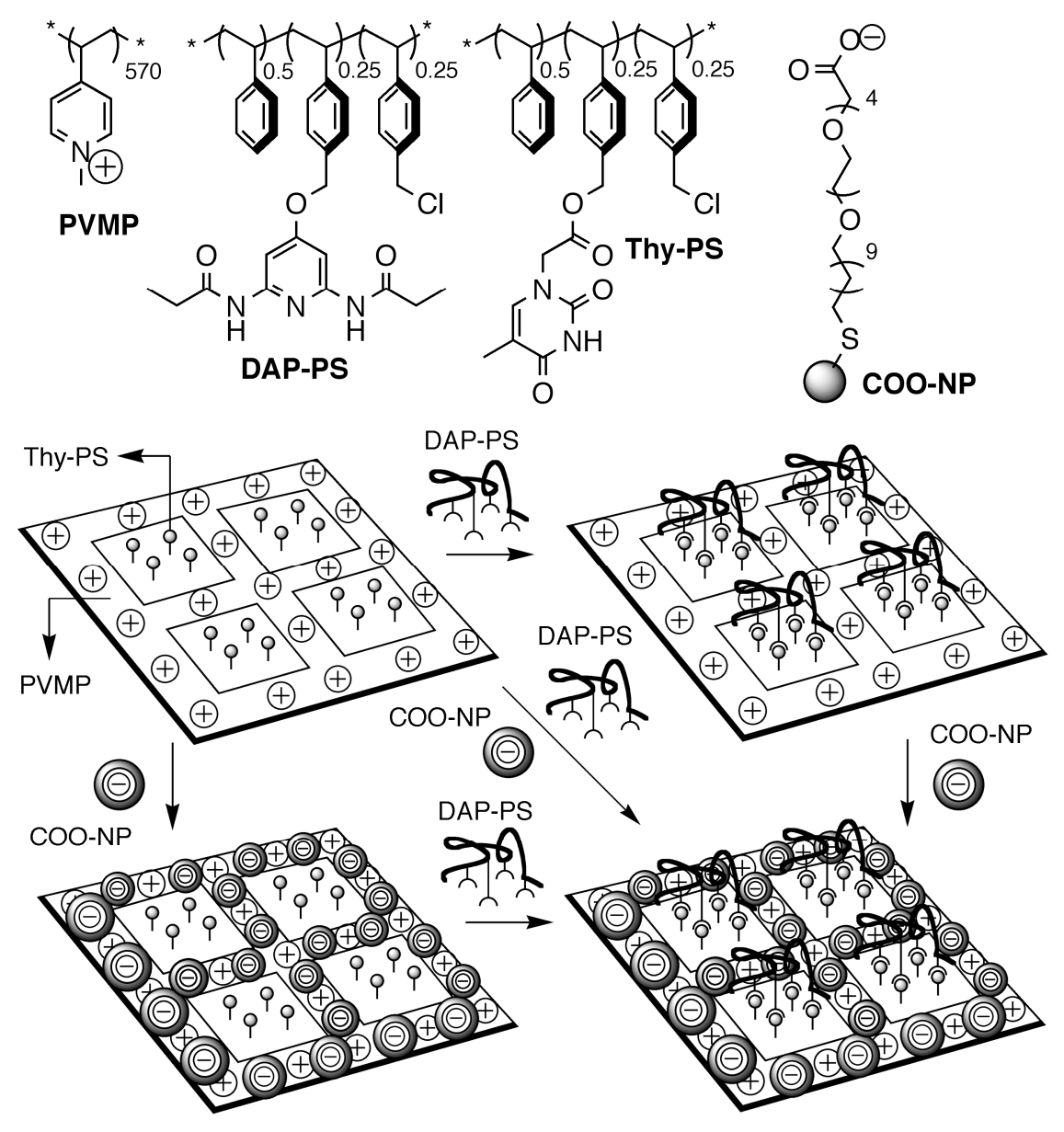

Scheme I-16. Self-sorting on patterned surfaces.

\subsubsection{Geometric Self-Sorting in DNA Self-Assembly.}

DNA is widely recognized for its ability to undergo high-fidelity pairing with its cognate strands based on AT and GC base pairing preferences and its use in studies of self-assembly. ${ }^{54}$ Chengde Mao's group recently examined whether geometric features of DNA assemblies could also be used to drive self-sorting processes (Scheme I-17). Accordingly, they designed two related DNA tiles namely a four point star I-65 and a three point star I-66 motif. The sticky single 
stranded ends of both DNA tiles are identical and self-complementary; the only difference is their relative geometrical relationship (e.g. $90^{\circ}$ versus $120^{\circ}$ ). The three pointed star gives rise to an extended hexagonal lattice structure I-67 as determined by AFM whereas the four-pointed star gives rise to extended square lattice structures I-68 $^{55}$ Interestingly, when both tiles are mixed (100:0 to 0:100 mole fraction) a single type of assembly is formed. Below a 30:70 ratio of I-65 to I-66 the hexagonal arrays dominate whereas above 30:70 the square arrays are mainly observed. The work demonstrates that rational design of DNA sequences can be used to augment the range of self-sorting systems that can be prepared. The ability to use DNA sequences in such systems is significant because it potentially enables replication and amplification events. ${ }^{56-59}$

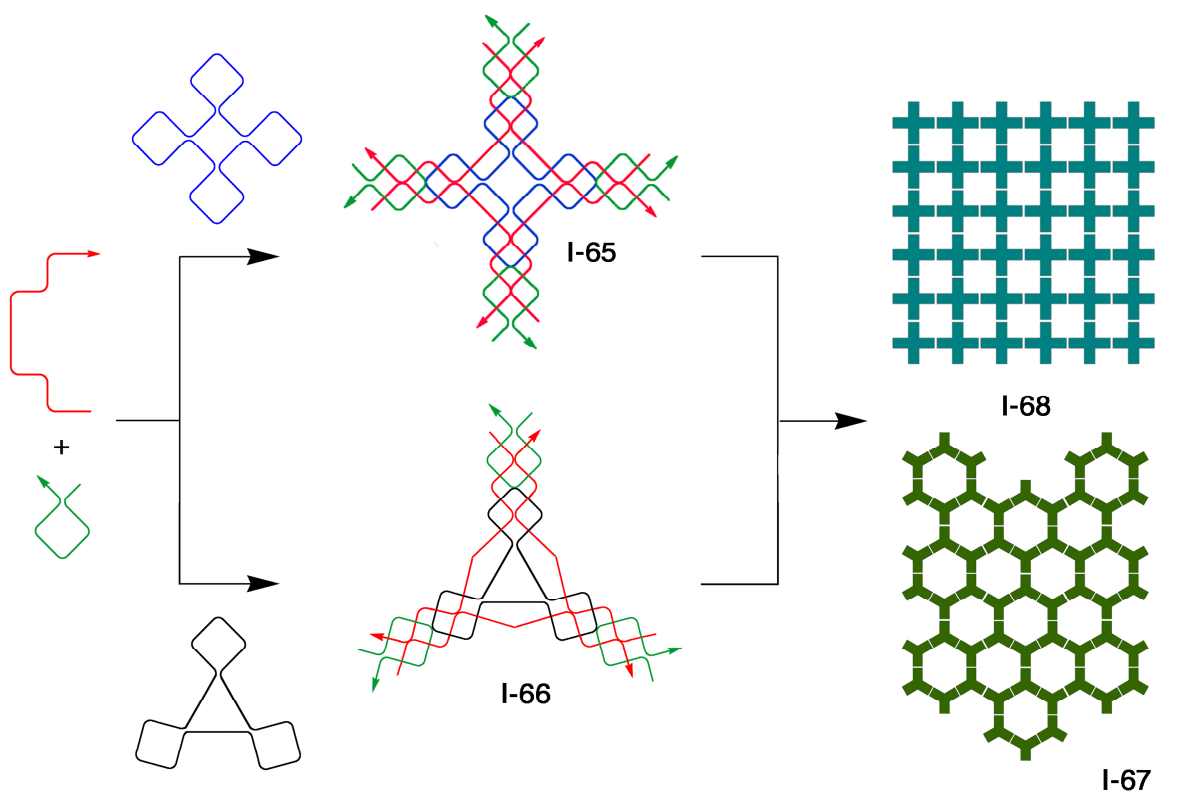

Scheme 17. Geometric self-sorting of DNA nanostructures. 


\subsubsection{Self-Sorting in Polymers.}

The group of Marcus Weck took inspiration from the self-sorting ability of one of the natural biopolymers - DNA - decided to develop the concept of selfsorting within synthetic polymers. Their goal was the creation of a "universal polymer backbone" that could be functionalized in a subsequent orthogonal noncovalent fashion with the hope that such a system would allow the rapid generation of polymeric materials for application in materials science, drug delivery, and biomimetic chemistry. For this purpose, Burd and Weck prepared two norbornene monomers and polymerized them (random, block, homo-polymers) by ring opening metathesis polymerization to yield $\mathbf{I - 6 9}$ with a range of molecular weights $(12-66$ $\mathrm{kD}$ ) and mole fractions (Scheme I-18). A series of ${ }^{1} \mathrm{H}$ NMR titrations of these polymers with the complementary H-bonding modules I-70 and I-71 were performed. Remarkably, Burd and Weck found that the association constant of I-70 and I-71 for their H-bonding partners were comparable regardless of whether those recognition units were monomeric, homopolymeric, or within a block or random or block copolymer. ${ }^{60}$ In addition to comparable association constants, the amide region of the ${ }^{1}$ H NMR spectra of I-72 under saturating conditions displayed the fingerprint of selfsorting - that the NMR spectra of the mixture is equal to the sum of its parts. In related work, Weck has employed other non-covalent interactions to derivatize the side chains of related polymer backbones and plans to use them in applications such as electronic materials, polymer mediated drug delivery, and tissue engineering. 


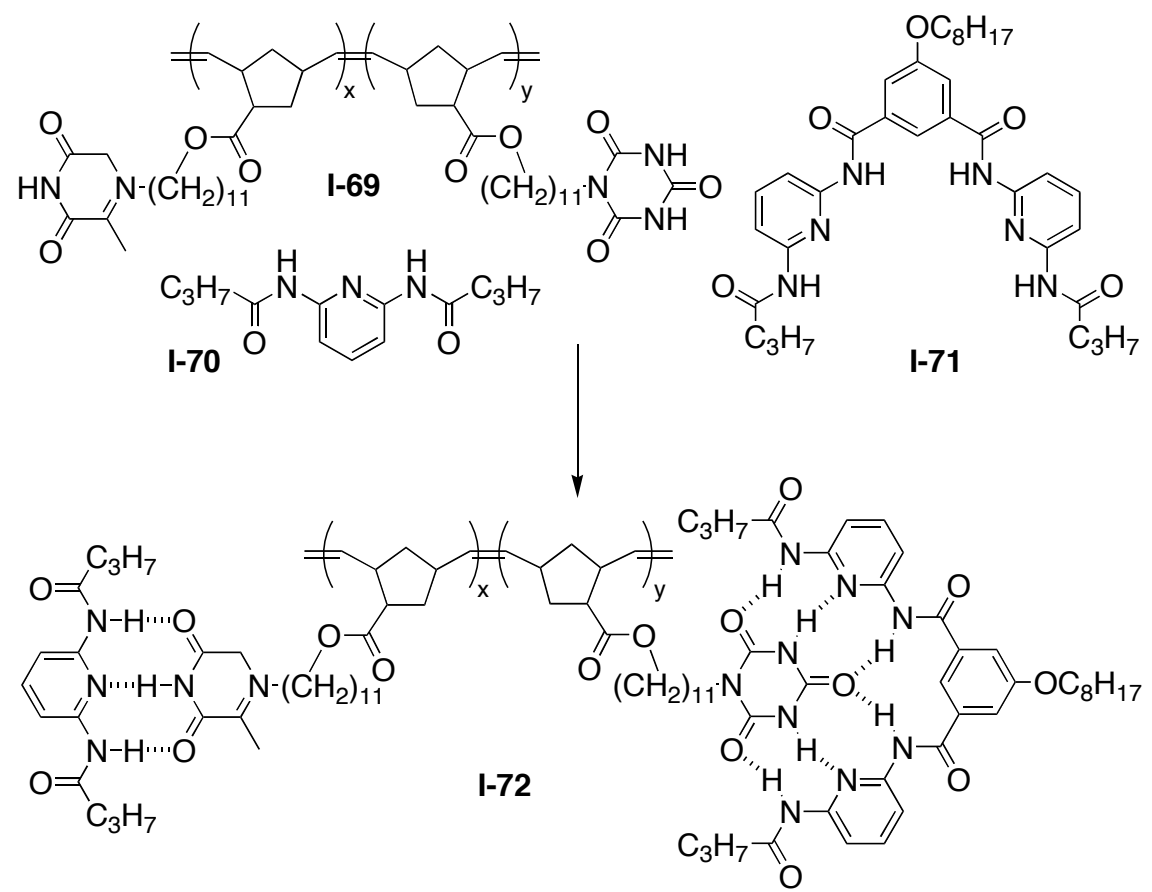

Scheme I-18. Self-sorting non-covalent functionalization of a universal polymer backbone.

\subsubsection{Self-Sorting Gels.}

The group of David Smith at the University of York decided to investigate the possibility of self-sorting during gelation processes. ${ }^{61}$ For their experiments they designed the lysine derived first and third generation dendritic peptides L-I-73, D- I73, and L- I-74 with the goal of deciphering the influence of head group size and chirality on gelation (Scheme I-19). The influence of head group size was studied with mixtures of L-I-73 and L-I-74. Very interestingly, the presence of L-I-73 did not affect the gelation temperature $\left(\mathrm{T}_{\text {gel }}\right)$ of the more effective L-I-74 gelator. Further studies with small angle $\mathrm{x}$-ray scattering and circular dichroism as the analytical tools 
once again indicate that L-I-73 does not influence the gelation of L-I-74 which is consistent with an efficient self-sorting process governed by the size of the dendritic head group (e.g. first versus third generation). The influence of molecular chirality on gelation mixtures of L-I-73 and D-I-73 were studied by measuring $\mathrm{T}_{\text {gel }}$ as a function of mole fraction of D-I-73. $\mathrm{T}_{\text {gel }}$ was found to be relatively invariant as a function of mole fraction which one again suggests a self-sorting in the gelation process. This conclusion is backed up by the results of CD measurements, which show a linear dependence on mole fraction. These experiments demonstrate that subtle molecular recognition pathways can be used to control self-organization on the macroscopic scales by efficient self-sorting processes. The work is expected to enable the creation of multifunctional nano-materials.

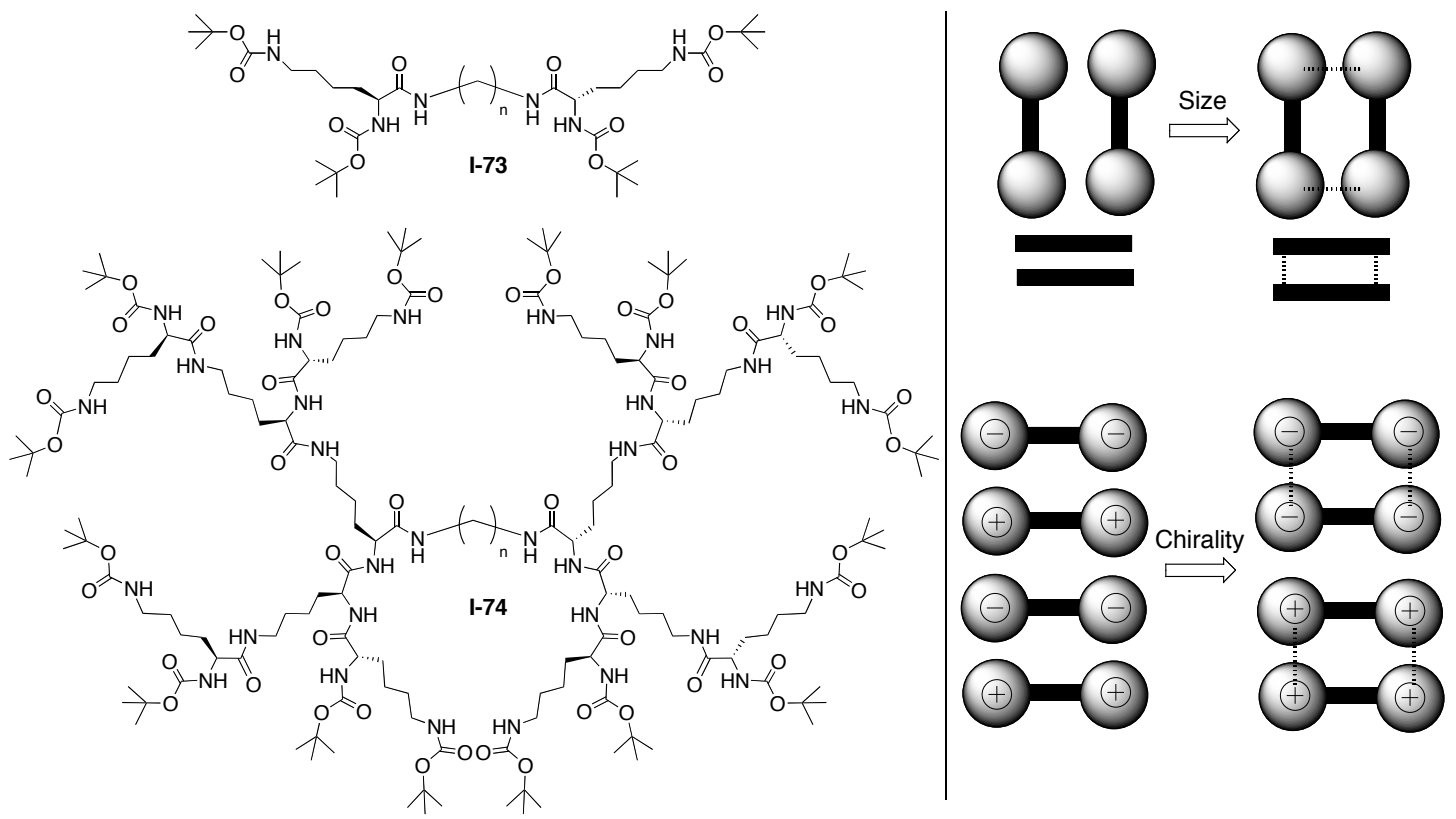

Scheme I-19. Self-sorting in dendritic peptide gels based on head group size or chirality. 


\subsection{Conclusion.}

This chapter focused on the preparation of self-sorting systems. In this area of research much inspiration is drawn from living systems whose complex web of recognition events is carefully orchestrated in both time and space and do not exhibit undesired cross-talk between different pathways. The components of natural and synthetic self-sorting systems display the ability to efficiently distinguish between self- and non-self and form a single set of structures in high-fidelity recognition processes. As such, self-sorting processes can be viewed as residing at the opposite end of a thermodynamic continuum from idealized dynamic combinatorial libraries in which all building blocks are incorporated without selectivity. To date, some covalent bond forming reactions (transesterification and imine formation) and a variety of non-covalent interactions have been shown to drive self-sorting processes including H-bonds, $\pi-\pi$ interactions, electrostatic interactions, solvophobic effects, and metal-ligand coordination. Such self-sorting processes can be divided into several categories including narcissistic versus social self-sorting and thermodynamic versus kinetic self-sorting.

A number of research groups have been involved in the development of pairs or sets of modules that undergo high-fidelity self sorting processes. Accordingly, a number of examples of both narcissistic and social self-sorting processes have been reported in which the system behaves as linear combination of its component parts. The development of sets of such orthogonal recognition pairs are of great potential 
utility in a variety of application areas including supramolecular polymer chemistry, gelators, DNA nanotechnology, and surface functionalization.

Most recently, several groups have been pushing self-sorting systems into new areas by introducing new concepts derived either from biological systems or desired for nanotechnology applications. For example, the groups of Severin and Nitschke have demonstrated a link between DCC and self-sorting processes and how the behavior of such combined systems differs from their separate sub-systems. Our group recently demonstrated how the high binding affinity and selectivity inherent in $\mathrm{CB}[\mathrm{n}]$ receptors can be used to drive the folding, forced unfolding, and refolding of a non-natural oligomer into four distinct conformations. This stepwise process transforms one well-defined self-sorted state into another by manipulating the overall free energy of the system by the addition of new components. Related concepts have been demonstrated by Kennan in coiled-coil systems and Nitschke in the triggered interconversion of sets of metal complexes. The development of systems that can be triggered to undergo interconversion between multiple well-defined states would be very useful in the preparation and interconnection of molecular machines. In order to endow synthetic self-sorting systems with some of the unique abilities of living systems (e.g living far from equilibrium) we recently demonstrated an example of kinetic self-sorting. For this purpose we used guests with multiple binding epitopes. Just like living systems - which reach equilibrium only in death - the individual binding epitopes of such guests orchestrate the approach toward equilibrium exhibited by the system. Much still remains to be done. For example, the incorporation of catalytic events into self-sorting systems - and the introduction of a non-natural 
energy source analogous to ATP - would allow manipulation of the overall free energy of the system which could be used to drive emergent processes. Beyond that, the physical boundaries of such systems must be defined to allow restricted (privileged) interaction with the outside world and must be engineered to remain far from equilibrium. Finally, if such non-natural self-sorting systems can be interfaced with natural systems that have the ability to replicate and evolve it might be possible to exert control over the corresponding biological system. 


\section{Chapter 2: Self-Sorting Molecular Clips. ${ }^{62}$}

\subsection{Introduction.}

As supramolecular chemistry has continued to develop following the pioneering work of Cram, Lehn, and Pedersen, the focus of much research in the area has shifted from an understanding of the fundamental aspects of noncovalent interactions, molecular recognition, and self-assembly and toward the use of such information to construct systems with function. ${ }^{2-4}$ For example, supramolecular chemistry has provided new approaches toward chemical sensing ensembles, discovery via dynamic combinatorial chemistry, drug delivery, membrane transport agents, DNA nanotechnology, control of otherwise unfavorable chemical reactions, and supramolecular polymers. ${ }^{10,12,54,63-70}$ In all of these applications, the ready availability of robust and easily functionalized modules capable of specific noncovalent interactions (e.g., $\pi-\pi$, metal-ligand, and H-bonding modules) with their targets in organic or aqueous solution has proved invaluable..$^{8,9,42,71-73}$ For example, quadruple hydrogen-bonding modules with high values of $K_{\mathrm{a}}$ have been used in the formation of high-molecular-weight supramolecular polymers. To date, however, the majority of such functional systems have been demonstrated to operate only in isolation and not as components of more complex chemical or biological systems.

In contrast to biologists, computer scientists, and engineers who embrace the need to study complex systems and appreciate the insights that may be gained from a 
systems level approach - chemists have been comparably slow to approach the study of complex multicomponent systems. This difference has begun to subside in recent years with the development of powerful new analytical tools that provide approaches to previously intractable problems. These groups of scientists drawn from numerous fields are beginning to define an area now known as systems chemistry. ${ }^{51,52,74-76}$ Systems chemists study complex multicomponent systems that exhibit emergent properties that go beyond those of their components. Some systems chemists take their inspiration from biology and design systems that exhibit properties such as selfreplication, ${ }^{77-83}$ whereas others add new components to existing biological systems in an attempt to control biological function. Other systems chemists take inspiration from technology and aim to integrate individual molecular devices into more complex molecular machines. ${ }^{13,72}$

We have based our entry into systems chemistry on the realization that all of these biological or technological systems depend critically on the availability of robust, easily functionalized supramolecular modules (vide supra) that operate not only in isolation but also as components of more complex systems. The network of binary and higher order interactions between the constituent molecules controls the behaviors of such systems. Rather than being governed by all possible (e.g., random) sets of interactions, such systems tend to organize themselves into a smaller number of sets of interacting molecules with interconnections between these sets that respond to external stimuli from their environment. As such, the ability of the constituents of such systems to efficiently distinguish between self and nonself is critical. We previously showed that a mixture comprising the components of eight well-known 
aggregates from the literature is capable of efficiently distinguishing between self and nonself even within the mixture on the basis of H-bond pattern and geometrical distribution and undergoes a high-fidelity self-sorting process. ${ }^{29}$ Subsequently, we have developed self-sorting systems based on host-guest interactions (social selfsorting), those that display well-defined kinetic and thermodynamic self-sorted states, and shown how such systems can be made to respond to chemical stimuli (e.g., guest addition or $\mathrm{pH}$ change). ${ }^{16,31,36,37,84}$ In this chapter we investigate the ability of a series of glycoluril-derived molecular clips $s^{21,85-93}$ (II-1 - II-11) several of which undergo tight dimerization $\left(K_{\mathrm{a}} \geq 10^{6} \mathrm{M}^{-1}\right)$ in $\mathrm{CDCl}_{3}$ solution to act as robust, functionalizable, self-sorting supramolecular modules. ${ }^{15,26,30,38-40,44,45,52,53,60,94-104}$ We envision that such supramolecular modules will greatly expand the toolbox available to systems chemists for the construction of complex and functional systems.

\subsection{Design and Synthesis of Molecular Clips.}

\subsubsection{Design of the Chemical Components Used in this Study.}

Chart II-1 shows the chemical structures of the eleven C-shaped methylene bridged glycoluril dimers used in this chapter. The key structural features in the design of molecular clips II-1 - II-11 were as follows. First, all twelve clips possess two roughly parallel aromatic walls separated by $\approx 7 \AA$ which define a cleft that promotes dimerization in chloroform driven by $\pi-\pi$ interactions. Even though $\pi-\pi$ interactions have preferred geometries (e.g. edge-to-face or offset face-to-face) we thought that dimeric molecular clips driven solely by $\pi-\pi$ interactions might exist as 
an ensemble of conformational isomers that differ in the relative orientation of the molecular clips with respect to each other (Figure II-1). Second, the rigid geometry of the molecular clips and the straightforward functionalization of their o-xylylene sidewalls was utilized to display H-bonding functional groups (e.g. amide, urea, oxamide) in well defined relative orientations. We envisioned that interactions between these $\mathrm{H}$-bonding arms could be used to drive dimerization in $\mathrm{CDCl}_{3}$ solution and also result in the selection of a single well-defined conformation from the conformational ensemble (Figure II-1) that satisfied the geometric requirements of both the H-bonding and $\pi-\pi$ interactions. 
Chart II-1. Chemical Structures of Molecular Clips Used in This Chapter.

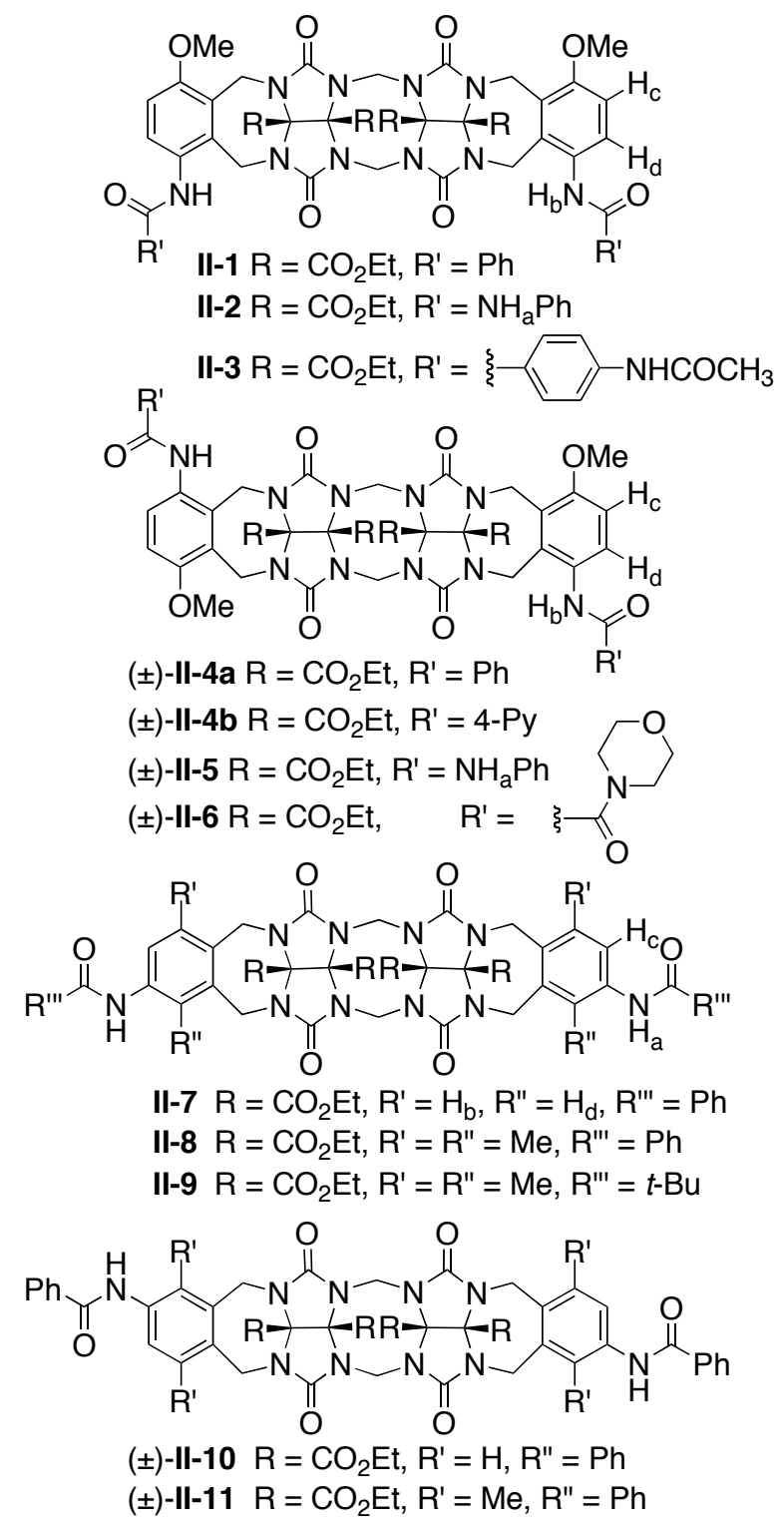



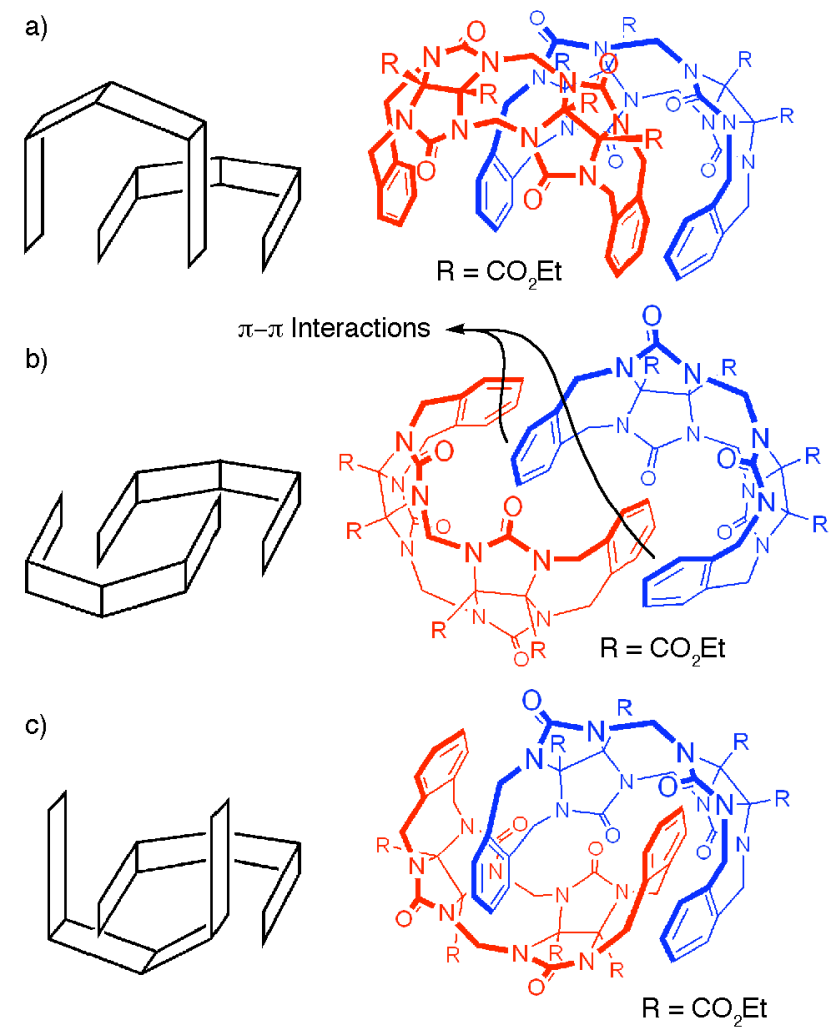

Figure II-1. Schematic representation of three members of the conformational ensemble open to dimeric molecular clips driven solely by $\pi-\pi$ interactions: a) perpendicular geometry, b) skewed geometry, and c) in register geometry.

\subsubsection{Synthesis of the Molecular Clips Bearing H-bonding Groups on Their Aromatic Ring.}

The synthesis of compounds II-1 - II-11 is shown in Scheme II-1. For the preparation of compounds II-1 - II-6, we used the previously reported dinitro compounds II-12 and ( \pm --II-13 as starting materials. ${ }^{17,20}$ The separate catalytic hydrogenation of II-12 and ( \pm )-II-13 to the corresponding air-sensitive diamines 
proceeded smoothly in dimethylformamide (DMF) as solvent. The crude diamines were transformed into the corresponding amides and ureas by reaction with acid chlorides and isocyanates in $\mathrm{CH}_{2} \mathrm{Cl}_{2}$ at room temperature to deliver II-1 - II-6 in 43 90\% yield. To prepare compounds II-7 and II-10, which contain amide substituents at the 4-position of the $o$-xylylene sidewalls we needed to prepare ( \pm )-II-16. For the synthesis of $( \pm)-\mathbf{I I}-16$, we first performed the free radical bromination of 4-nitro-oxylene using $N$-bromosuccinimide in 1,2-dichloroethane which gave II-14 in 47\% yield. ${ }^{105}$ Alkylation of II-15 with compound II-14 ( $t$-BuOK, DMSO) gave ( \pm )-II-16 in $22 \%$ yield. Compound $( \pm)-\mathbf{I I}-\mathbf{1 6}$ was dimerized by acid-catalysed condensation with paraformaldehyde in the presence of $p$-toluenesulfonic acid (PTSA) in $\mathrm{ClCH}_{2} \mathrm{CH}_{2} \mathrm{Cl}$ to yield $\mathbf{I I - 1 7}$ and $( \pm)-\mathbf{I I}-\mathbf{1 8}$ in $23 \%$ and $20 \%$ yield, respectively. ${ }^{17}$ After chromatographic separation, diastereomers II-17 and ( \pm )-II-18 were separately reduced and then acylated with benzoyl chloride to give II-7 and ( \pm ) II-10 in 73\% and $76 \%$ yield, respectively.

Compounds II-8, II-9 and ( \pm )-II-11 were synthesized by related procedures. Somewhat surprisingly, we found that heating II-19 with PTSA in $p$-xylene gave II20 by extrusion of one of the $\mathrm{CH}_{2} \mathrm{OCH}_{2}$ bridges in $36 \%$ yield. Acid catalysed condensation of compound II-20 with paraformaldehyde gave dimer II-21 in 78\% yield. Compound II-21 was nitrated (TFA, $\mathrm{HNO}_{3}$ ) to give a mixture of diastereomers II-22 and ( \pm -II-23 in $42 \%$ and $38 \%$ yield, respectively. After chromatographic separation, diastereomers $\mathbf{I I - 2 2}$ and ( \pm -)-II-23 were separately reduced and then acylated with the appropriate acid chloride to give II-8, II-9 and ( \pm )-II-11 in $65-$ $75 \%$ yield. 


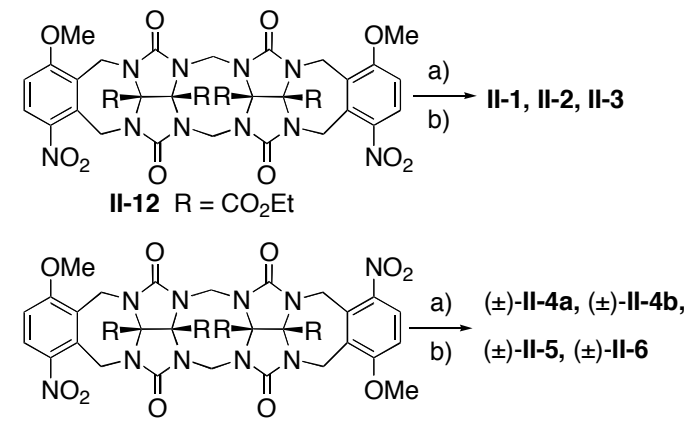

(土)-II-13 R $=\mathrm{CO}_{2} \mathrm{Et}$

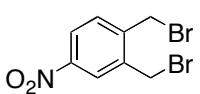

II-14

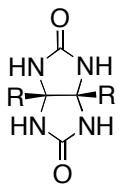

II-15 R $=\mathrm{CO}_{2} \mathrm{Et}$
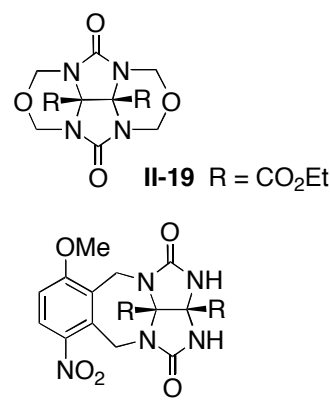

(土)-II-25 R $=\mathrm{CO}_{2} \mathrm{Et}$

(15

(土)-II-16 R $=\mathrm{CO}_{2} \mathrm{Et}$

a), b) $\left[\begin{array}{ll}\mathrm{Il}-17 \mathrm{R}=\mathrm{CO}_{2} \mathrm{Et}, \mathrm{R}^{\prime}=\mathrm{NO}_{2} \\ \mathrm{II}-7 \mathrm{R}=\mathrm{CO}_{2} \mathrm{Et}, \mathrm{R}^{\prime}=\mathrm{NHCOPh}\end{array}\right.$

a), b) $\left[( \pm)\right.$-II-18 $\mathrm{R}=\mathrm{CO}_{2} \mathrm{Et}, \mathrm{R}^{\prime}=\mathrm{NO}_{2}$

$$
\longrightarrow \mathrm{NI} \mathrm{R}=\mathrm{CO}_{2} \mathrm{Et}, \mathrm{R}^{\prime}=\mathrm{NHCOPh}
$$

I-19

(土)-II-20 R $=\mathrm{CO}_{2} \mathrm{Et}$

\footnotetext{
f) $\square$ II-21 $\mathrm{R}=\mathrm{CO}_{2} \mathrm{Et}, \mathrm{R}^{\prime}=\mathrm{H}$

a), b) $\square$ II-22 R= $\mathrm{Il}=\mathrm{CO}_{2} \mathrm{Et}, \mathrm{R}^{\prime}=\mathrm{NO}_{2}$

$\rightarrow$ II-9 R $=\mathrm{CO}_{2} \mathrm{Et}, \mathrm{R}^{\prime}=\mathrm{NHCO} t-\mathrm{Bu}$
}

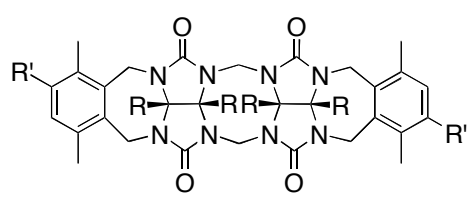

f) $\square$ II-21 $\mathrm{R}=\mathrm{CO}_{2} \mathrm{Et}, \mathrm{R}^{\prime}=\mathrm{H}$

a), b)

Scheme II-1. Synthesis of molecular clips II-1 - II-11. Conditions: a) $\mathrm{Pd} / \mathrm{C}, \mathrm{H}_{2}$, DMF, b) PhNCO or R' $\mathrm{COCl}, \mathrm{Et}_{3} \mathrm{~N}, \mathrm{CH}_{2} \mathrm{Cl}_{2}$, c) II-14, $t$-BuOK, DMSO, d) PTSA, $\left(\mathrm{CH}_{2} \mathrm{O}\right)_{n}, \mathrm{ClCH}_{2} \mathrm{CH}_{2} \mathrm{Cl}$, reflux, e) $p$-xylene, reflux, f) TFA, $\mathrm{HNO}_{3}$.

\subsection{Self-assembly of Molecular Clips.}

\subsubsection{Molecular Clips Form Homodimers in $\mathrm{CDCl}_{3}$.}

The ${ }^{1} \mathrm{H}$ NMR spectra of II-1, II-2, ( \pm )-II-4a, and ( \pm$)-\mathbf{I I}-\mathbf{5}$ in DMSO- $d_{6}$ show a single set of resonances due to the presence of two symmetry equivalent halves which indicates these molecules are monomeric in DMSO- $d_{6}$. In $\mathrm{CDCl}_{3}$, however, we 
observe a doubling of resonances in their ${ }^{1} \mathrm{H}$ NMR spectra along with significant anisotopic effects, which suggests that these molecules exist as dimers (Figure II-2). For example, although only two N-H resonances $\left(\mathrm{H}_{\mathrm{a}}\right.$ and $\left.\mathrm{H}_{\mathrm{b}}\right)$ were observed in DMSO- $d_{6}$ for II-2, we observed four resonances in $\mathrm{CDCl}_{3}\left(\mathrm{H}_{\mathrm{a}}, \mathrm{H}_{\mathrm{a}}, \mathrm{H}_{\mathrm{b}}\right.$ and $\left.\mathrm{H}_{\mathrm{b}}\right)$, which correspond to the two different $\mathrm{N}-\mathrm{H} \cdots \mathrm{O}$ H-bonds in homodimer II-2•II-2. Similarly, in the monomeric form the aromatic protons of II-2 $\left(\mathrm{H}_{\mathrm{c}}\right.$ and $\left.\mathrm{H}_{\mathrm{d}}\right)$ give rise to single set of resonances due to the presence of a mirror plane in the molecule. When II-2 undergoes dimerization to yield II-2•II-2 this mirror plane no longer exists. One set of aromatic rings resides in the interior of the dimer whereas the other set is on the exterior. The chemical shift of the aromatic protons $\left(\mathrm{H}_{\mathrm{c}^{\prime}}\right.$, and $\left.\mathrm{H}_{\mathrm{d}^{\prime}}\right)$ on the internal aromatic wall of II-2-II-2 resonate upfield relative to the protons of the external aromatic ring $\left(\mathrm{H}_{\mathrm{c}}\right.$, and $\left.\mathrm{H}_{\mathrm{d}}\right)$ because they are in the shielding region of two neighboring aromatic rings. Similar diagnostic features were observed in the ${ }^{1} \mathrm{H}-$ NMR of II-1・II-1, (+)-II-4a・(-)-II-4a, and (+)-II-5•(-)-II-5 in $\mathrm{CDCl}_{3}$.

We performed ${ }^{1} \mathrm{H}$ NMR dilution experiments to determine the self-association constant $\left(\mathrm{K}_{\mathrm{s}}\right)$ for the dimeric aggregation. Remarkably, the chemical shifts observed for dimers II-1•II-1, II-2•II-2, (+)-II-4a•(-)-II-4a, and (+)-II-5•(-)-II-5 do not change when the concentration is decreased from $10 \mathrm{mM}$ and $50 \mu \mathrm{M}$, indicating their high thermodynamic stability (Figure II-3). If we assume that we could detect changes in the chemical shifts due to $10 \%$ monomer, then we can place a lower limit on the of the value of $\mathrm{K}_{\mathrm{s}}\left(\mathrm{K}_{\mathrm{s}}>9 \times 10^{5} \mathrm{M}^{-1}, \Delta \mathrm{G}<-8.1 \mathrm{kcal} \mathrm{mol}^{-1}\right)$. Such a high thermodynamic stability was not anticipated given that these dimeric aggregates benefit from a mere two H-bonds. We surmise that the high thermodynamic stability 
of these aggregates is due to the cumulative effect of $\pi-\pi$ interactions and H-bonds. The concentration invariance and distinct pattern of anisotropic effects in the ${ }^{1} \mathrm{H}$ NMR spectra of dimers II-1•III-1, II-2•II-2, (+)-II-4a・(-)-III-4a, and (+)-II-5•(-)-II-5 indicate that the combined driving force and geometrical constraints of $\pi-\pi$ interactions and $\mathrm{H}$-bonds are capable of selecting a single member of the complex conformational ensemble open to dimeric molecular clips (Figure II-1).

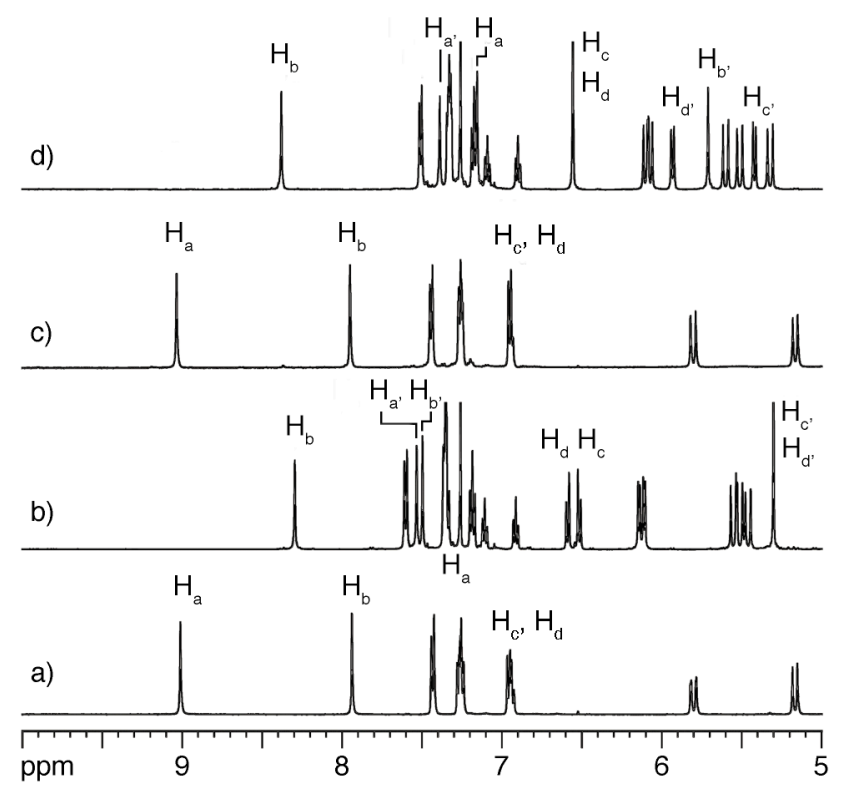

Figure II-2. Portion of the ${ }^{1} \mathrm{H}$ NMR (500 MHz, RT) spectrum recorded for: a) II-2 in DMSO- $d_{6}$, b) $\mathbf{I I - 2} \cdot \mathbf{I I}-\mathbf{2}$ in $\mathrm{CDCl}_{3}$, c) $( \pm)-\mathbf{I I}-\mathbf{5}$ in DMSO- $d_{6}$, d) $(+)-\mathbf{I I}-\mathbf{5} \bullet(-)-\mathbf{I I}-\mathbf{5}$ in $\mathrm{CDCl}_{3}$. 


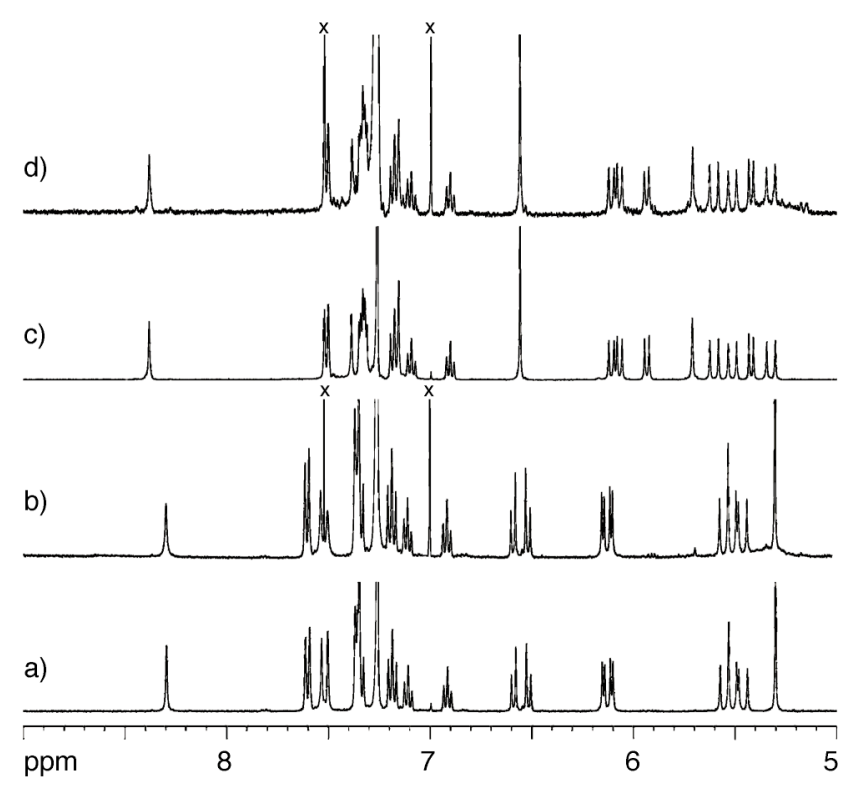

Figure II-3. Portion of the variable concentration ${ }^{1} \mathrm{H}$ NMR spectrum recorded for a (400 MHz, $\left.\mathrm{CDCl}_{3}, \mathrm{RT}\right)$ : a) $5 \mathrm{mM}$ of II-2・II-2, b) $50 \mu \mathrm{M}$ of II-2・II-2, c) $5 \mathrm{mM}$ of (+)II-5•(-)-II-5 and d) $50 \mu \mathrm{M}$ of (+)-II-5•(-)-II-5.

\subsubsection{Concept of Geometrical Match and Mismatch.}

As described above, compounds II-1, II-2, ( \pm )-II-4a and ( \pm )-II-5 undergo strong homodimerization in $\mathrm{CDCl}_{3}$ driven by $\pi-\pi$ interactions and $\mathrm{H}$-bonds. We previously investigated their recognition behavior in binary mixtures of II-1 and ( \pm )II-4a or II-2 and ( \pm )-II-5 and observed the phenomenon of self-sorting. ${ }^{21} \mathrm{We}$ attribute this high preference for self-sorting - heterodimers $(( \pm)-\mathbf{I I}-\mathbf{1} \bullet \mathbf{I I}-\mathbf{4 a}$ or $( \pm)$-II2-II-5) were not observed by ${ }^{1} \mathrm{H}$ NMR - to a geometrical mismatch between the Hbonding arms II-1 and ( \pm )-II-4a or II-2 and ( \pm )-II-5 in the hypothetical heterodimers (Figure II-4). The hypothetical heterodimer $( \pm)-\mathbf{I I}-\mathbf{1} \cdot \mathbf{I I}-\mathbf{4 a}$, for example, benefits 
from $\pi-\pi$ interactions and only a single $\mathrm{H}-$ bond as opposed to $\pi-\pi$ interactions and at least two H-bonds in homodimers II-1•II-1 and (+)-II-4a•(-)-II-4a (Figure II-4). Conversely, when an equimolar mixture of II-1 and II-2 with common spatial distribution of their $\mathrm{H}$-bonding arms was prepared in $\mathrm{CDCl}_{3}$, we observed a roughly statistical mixture of homodimers II-1•II-1 and II-2•II-2 and heterodimer ( \pm -)-II-1 $\bullet \mathbf{I I}-$ 2 by ${ }^{1} \mathrm{H}$ NMR (Figure II-5). Similarly, when we analyzed a mixture of ( \pm )-II-4a and $( \pm)-$ II-5 by ${ }^{1} \mathrm{H}$ NMR, a mixture of two heterochiral homodimers ((+)-II-4a・(-)-II-4a and $(+)-\mathbf{I I}-5 \cdot(-)-\mathbf{I I}-5)$ and racemic mixture of heterochiral heterodimers $((+)-\mathbf{I I}-$ 4a・(-)-II-5 and (-)-II-4a・(+)-II-5) were observed. Based on this result we hypothesized that subtle differences in the spatial orientation of H-bonding groups on the edges of the $o$-xylylene rings of these molecular clips might endow these compounds with the ability to efficiently distinguish between self and non-self within binary and higher order mixtures. 


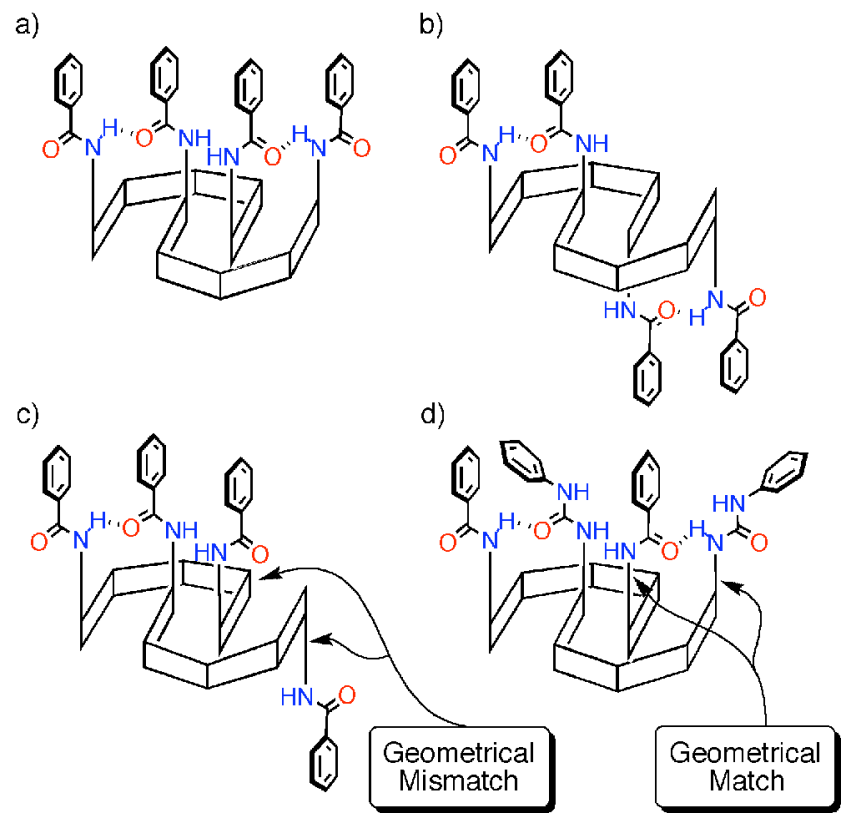

Figure II-4. Schematic representation of geometrical match versus geometrical mismatch: a) II-1•II-1 and b) (+)-II-4a・(-)-II-4a, c) hypothetical diastereomeric aggregate formed by $( \pm)-\mathbf{I I}-\mathbf{1} \bullet \mathbf{I I}-4 \mathbf{a}$, and d) racemic heterodimer $( \pm)-\mathbf{I I}-\mathbf{1} \bullet \mathbf{I I}-\mathbf{2}$.

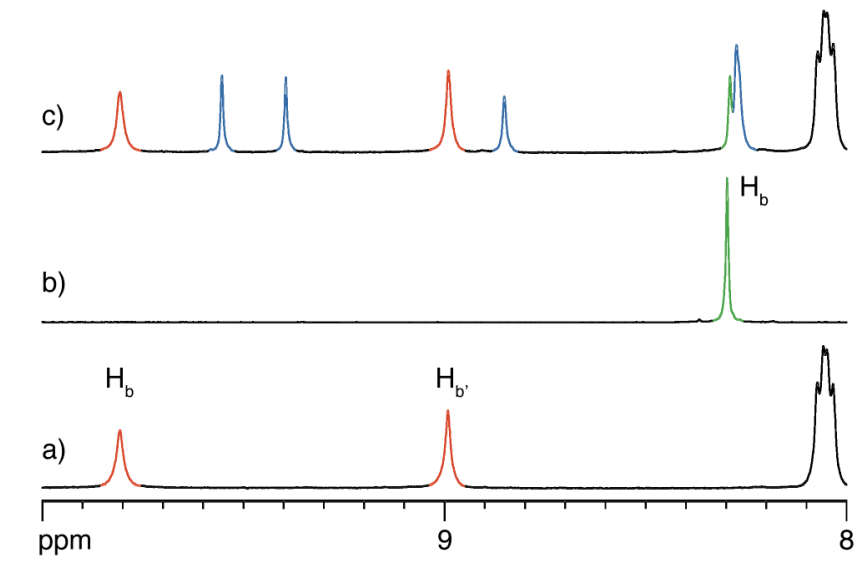

Figure II-5. Portion of the ${ }^{1} \mathrm{H}$ NMR $\left(2.5 \mathrm{mM}, 500 \mathrm{MHz}, \mathrm{CDCl}_{3}, \mathrm{RT}\right)$ spectrum recorded for: a) II-1•II-1, b) II-2•II-2, and c) a mixture of II-1•II-1, II-2•II-2, and $( \pm)-I I-1 \bullet I I-2$. Resonances for the amide N-H groups are color coded as follows: II1•II-1, red; II-2•II-2, green; and (土)-II-1•II-2, blue. 


\subsubsection{X-ray Crystal Structures of II-1, II-2, $( \pm)-\mathbf{I I}-4 \mathbf{b}$ and $( \pm)-\mathbf{I I}-5$.}

Although the ${ }^{1} \mathrm{H}$ NMR experiments mentioned in the previous section clearly support the formation of dimeric aggregates, additional evidence of dimer formation was obtained from the X-ray crystal structures of II-1, II-2, $( \pm)-\mathbf{I I}-\mathbf{4 b}$ and $( \pm)-\mathbf{I I}-\mathbf{5}$. Interestingly, all four molecular clips form dimers in solid state. All four dimers - II1•II-1, II-2•II-2, (+)-II-4b•(-)-II-4b, and (+)-II-5•(-)-II-5 - benefit from $\pi-\pi$ interactions between the substituted $o$-xylylene walls. All of them also benefit from two H-bonds between the external amide (urea) N-H groups and internal amide (urea) $\mathrm{C}=\mathrm{O}$ groups and secondary electrostatic interactions. Several other features of the geometries of these dimers in the solid state are noteworthy. For example, the pendant benzoyl groups in dimers II-1•II-1 and II-2•II-2 are displayed in a nearly collinear orientation on a single face of the dimer (Figure II-6a, II-6b). An additional level of complexity is present for the case of chiral but racemic molecular clips $( \pm)$ II-4b and ( \pm )-II-5 where both homochiral dimerization (e.g. $(+)-\mathbf{I I}-\mathbf{4 b} \bullet(+)-\mathbf{I I}-\mathbf{4 b}$ and $(-)-\mathbf{I I}-\mathbf{4 b} \cdot(-)-\mathbf{I I}-\mathbf{4 b})$ or heterochiral dimerization (e.g. (+)-II-4b•(-)-II-4b) are

conceivable. ${ }^{38,102,106,107}$ In the crystal we only observe the formation of heterochiral dimers (Figure II-6c, II-6d). We attribute this result to the geometrical mismatch between the H-bonding groups in the hypothetical homochiral homodimer. All these observations in the solid state determined by X-ray crystallography are consistent with their ${ }^{1} \mathrm{H} \mathrm{NMR}$ spectra in $\mathrm{CDCl}_{3}$ and we suggest that II-1, II-2, ( \pm )-II-4a, and ( \pm )-II-5 are isostructural in the solid state and in solution. ${ }^{93}$ The X-ray crystal structures of II-1, II-2, ( \pm )-II-4b, and $( \pm)-\mathbf{I I}-5$ also helps us to rationalize the behavior of these molecules within binary and higher order mixtures (vide infra). 

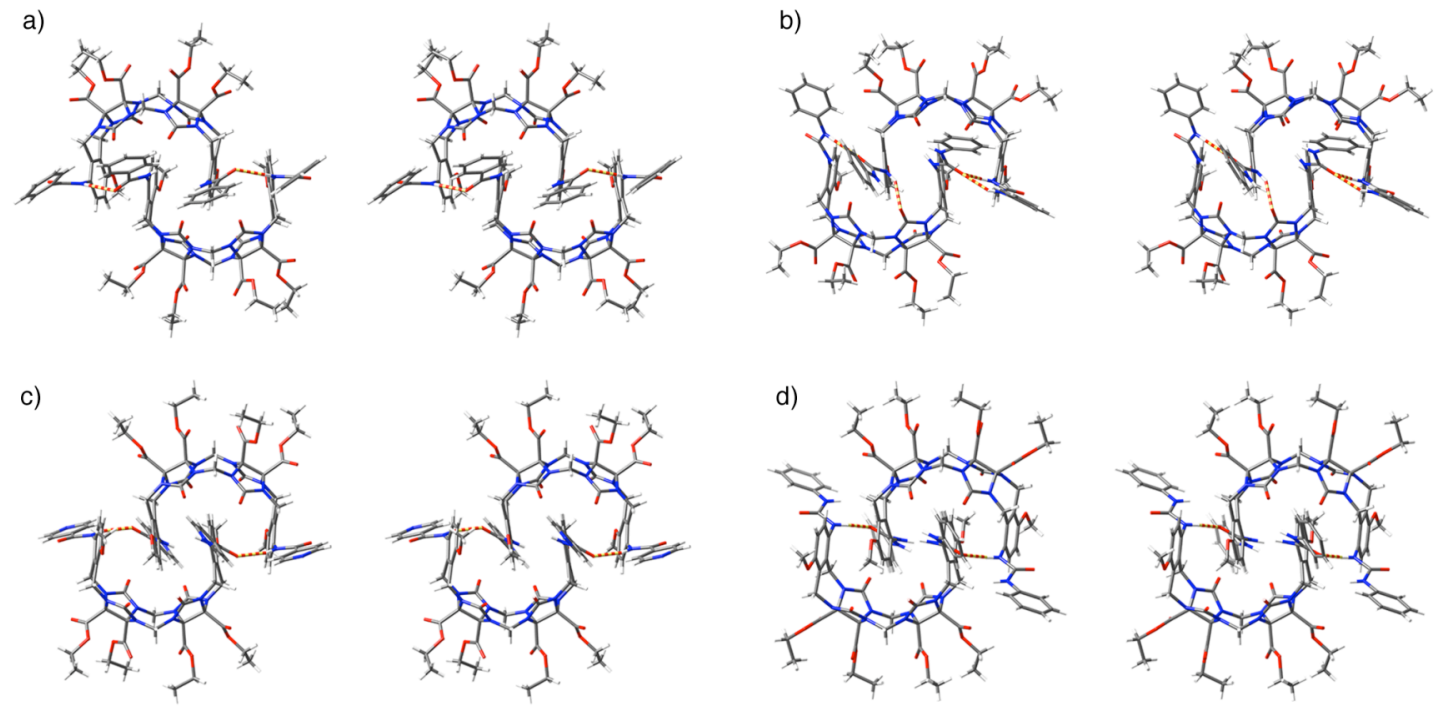

Figure II-6. Cross-eyed stereoviews of the molecular structures of: a) II-1•II-1, b) II-2•II-2, c) (+)-II-4b•(-)-II-4b, and d) (+)-II-5•(-)-II-5 in the crystal. Color coding: C, gray; H, white; N, blue; O, red; H-bonds, yellow-red striped.

\subsection{Three- and Four- Component Self-Sorting Systems of Molecular Clips.}

\subsubsection{Design Aspects of New Molecular Clips.}

The high selectivity we observed during the self-recognition of II-2 and ( \pm )II-5 in a binary mixture of molecular clips and the lack of self-selectivity for mixtures of II-1 and II-2 or ( \pm )-II-4a and ( \pm )-II-5 inspired us to consider situations that fall between these two extreme cases guided by a series of questions. What happens to the strength of dimeric aggregation and fidelity of self-sorting when the H-bonding groups are moved around the edges of the substituted $o$-xylylene walls? Will more complex mixtures of molecular clips (e.g. three or four components) constitute self- 
sorting systems if each clip has a distinct spatial arrangement of its H-bonding arms? We were also interested to investigate the effect of various structural modifications (e.g. substituents, steric bulk, number and pattern of H-bonds) on self-assembly and self- versus non-self recognition behavior in a multi-component mixture that would impact their use as components for advanced applications (e.g. stimuli responsive supramolecular polymers) and the topology of their network interactions ${ }^{101}$ when utilized as parts of more complex (bio)molecular machines. To address these questions we synthesized compounds II-7 - II-11 and studied their self-association and self-sorting properties in $\mathrm{CDCl}_{3}$ solution.

\subsubsection{Self-Assembly of II-7 in $\mathrm{CDCl}_{3}$ solution and solid state.}

As a first attempt to assess the ability of more subtle geometrical changes to direct self-sorting processes we prepared II-7, which as opposed to II-1 - II-6 has $\mathrm{PhCONH}$ groups on the tips of its $o$-xylylene sidewalls. Similar to II-1, II-2, ( \pm )-II4a and ( \pm )-II-5, compound II-7 exhibits a single set of resonances in its ${ }^{1} \mathrm{H}$ NMR spectrum in DMSO- $d_{6}$ - indicative of a monomer - but two sets of resonances in $\mathrm{CDCl}_{3}$ indicative of dimer formation (Figure II-8). Dimeric aggregate II-7•II-7 does not undergo significant dissociation down to $50 \mu \mathrm{M}$ (Figure II-9), which establishes a high thermodynamic stability $\left(\mathrm{K}_{\mathrm{a}}>10^{6} \mathrm{M}^{-1}\right)$ for this dimer. We were fortunate to obtain an X-ray crystal structure of II-7•II-7, which shows a number of interesting features (Figure II-7). In the dimeric aggregate II-7•II-7, the clips are skewed relative to one another (see Figure II-1b) in such a way that the aggregate benefits 
from $\pi-\pi$ interactions as well as four H-bonds (Figure II-10). The amide N-H groups on the internal aromatic rings form $\mathrm{H}$-bonds with the ureidyl $\mathrm{C}=\mathrm{O}$ of a glycoluril ring on the opposing clip whereas the external amide $\mathrm{N}-\mathrm{H}$ groups form $\mathrm{H}-$ bonds with the internal amide $\mathrm{C}=\mathrm{O}$ groups. Based on the distinct geometries observed for II-2•II-2, (+)-II-5•(-)-II-5, and II-7•II-7 we wondered whether a mixture of these three molecular clips would undergo high fidelity self-sorting.
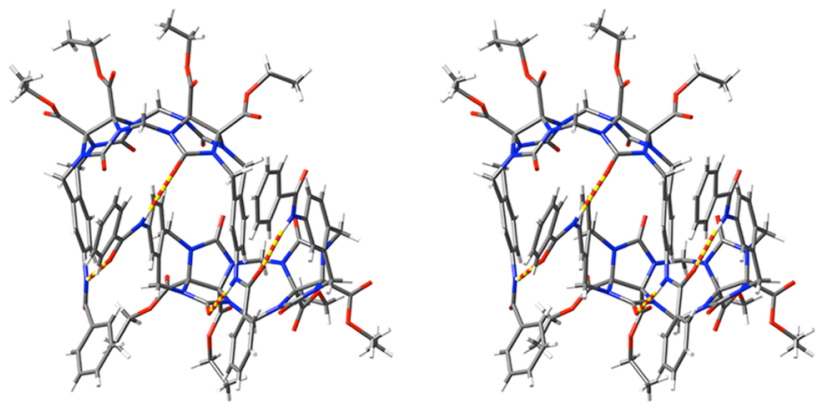

Figure II-7. Cross-eyed stereoviews of the molecular structures of: II-8・II-8 in the crystal. Color coding: C, gray; H, white; N, blue; O, red; H-bonds, yellow-red striped.

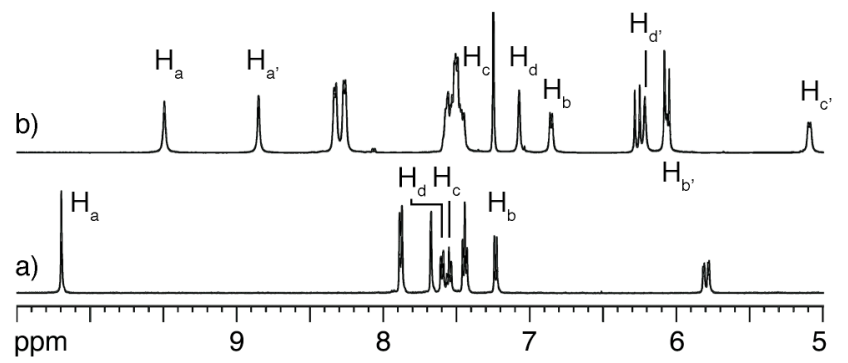

Figure II-8. Portion of the ${ }^{1} \mathrm{H}$ NMR (500 MHz, RT) spectrum recorded for : a) II-7 in DMSO- $d_{6}$, b) $\mathbf{I I}-7 \cdot \mathbf{I I}-7$ in $\mathrm{CDCl}_{3}$. 


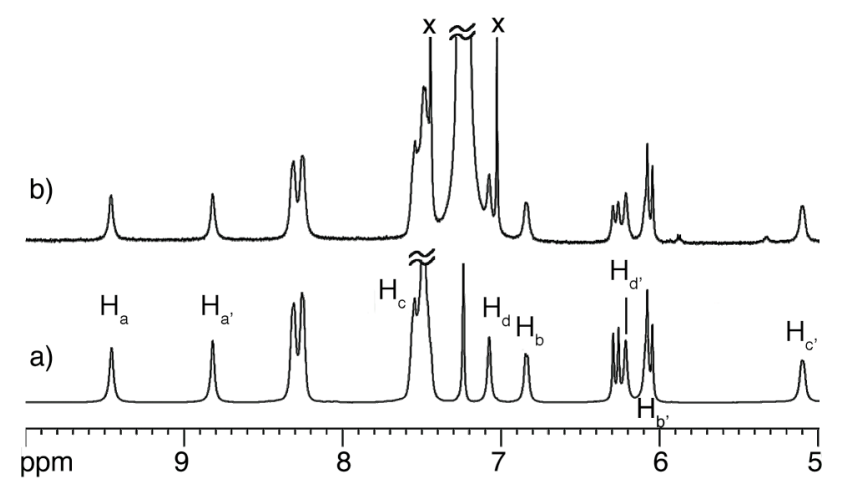

Figure II-9. Portion of the ${ }^{1} \mathrm{H}$ NMR spectrum recorded for II-7•II-7 (400 MHz, $\left.\mathrm{CDCl}_{3}, \mathrm{RT}\right)$ : a) $10 \mathrm{mM}$, and b) $50 \mu \mathrm{M}\left(\mathrm{x}={ }^{13} \mathrm{CHCl}_{3}\right)$.
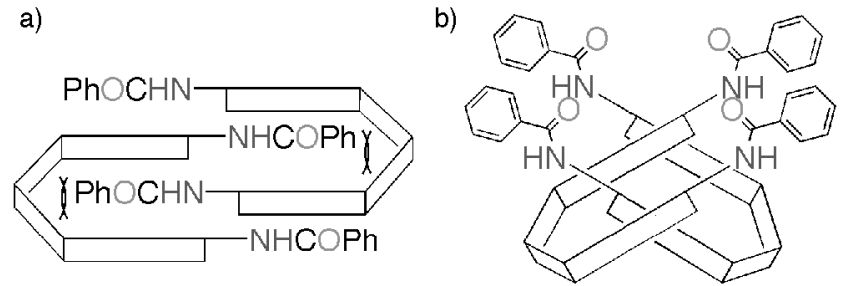

Figure II-10. Schematic representation of the shapes of II-7-II-7: a) steric interaction in the head-to-head form, and b) a skewed geometry that avoids steric interactions.

\subsubsection{Three Component Self-Sorting Systems.}

The high level of enantioselectivity exhibited by (+)-II-5•(-)-II-5 and skewing observed in the X-ray structure of II-7•II-7 led us to combine these clips with II-2• II-2 for the next set of experiments. Initially we examined the behavior of the three binary mixtures (e.g. II-2• III-2 and (+)-II-5•(-)-II-5 or II-2• II-2 and II7•II-7 or (+)-II-5•(-)-II-5 and II-7•II-7) and were delighted to observe a simple 
superimposition of the ${ }^{1} \mathrm{H}$ NMR spectra of the constituent homodimeric aggregates in each case. These results indicate that these three molecular clips possess the ability to efficiently distinguish between self and non-self. Accordingly, we next constructed the three component mixture comprising II-2, ( \pm )-II-5, and II-7 (or II-1, ( \pm )-II-5, and II-7) and observed an ${ }^{1} \mathrm{H}$ NMR spectrum consistent with the formation of a selfsorted mixture of the three homodimeric aggregates II-2•II-2, (+)-II-5•(-)-II-5, and II-7•II-7 (or II-1•II-1, (+)-II-5•(-)-II-5, and II-7•II-7; Figure II-11 and II-12). These results demonstrate that simply by choosing molecular clips that possess different geometrical arrangements of their H-bonding arms it is possible to achieve high fidelity self-sorting even within a complex mixture. We expect that such mutually orthogonal H-bonding modules have broad potential as triggered components of more complex molecular machines. ${ }^{13,72}$

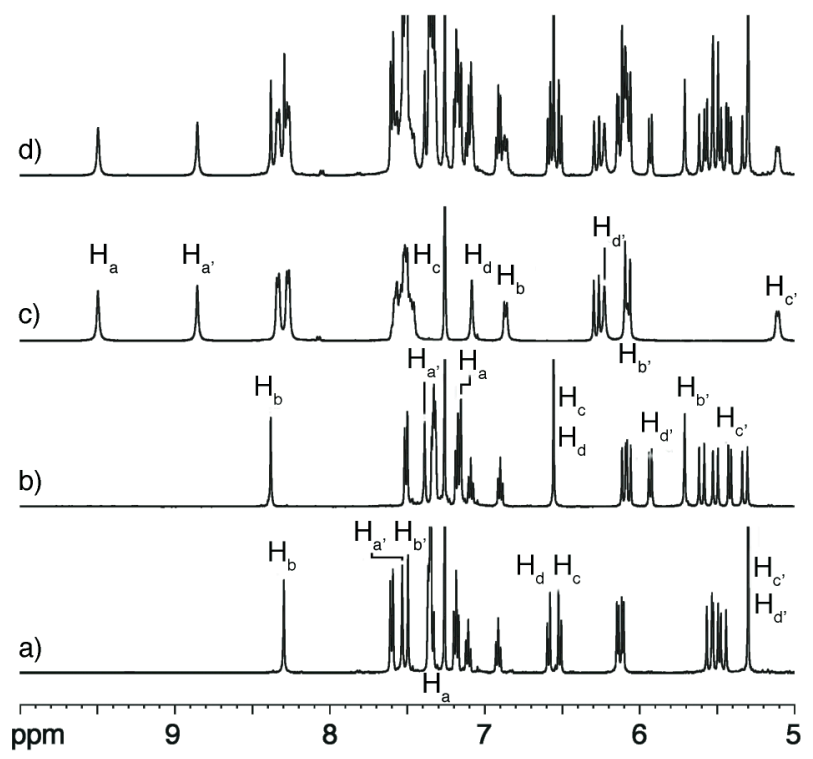

Figure II-11. ${ }^{1} \mathrm{H}$ NMR spectra $(500 \mathrm{MHz}, 298 \mathrm{~K})$ for: a) II-2•II-2, b) (+)-II-5•(-)II-5, c) II-7•II-7, and d) a mixture of II-2•II-2, (+)-II-5•(-)-II-5, and II-7•II-7. 


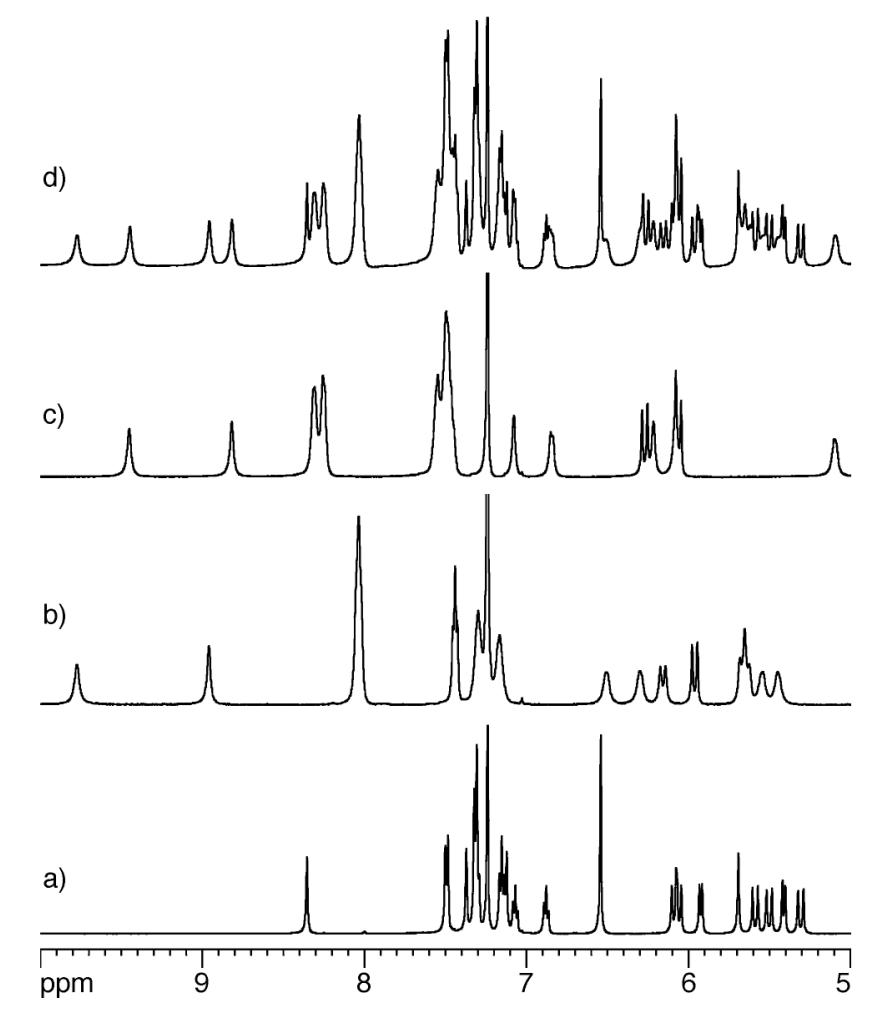

Figure II-12. ${ }^{1} \mathrm{H}$ NMR spectra (400 MHz, $\left.298 \mathrm{~K}\right)$ for: a) (+)-II-5•(-)-II-5, b) II-1• II-1, c) II-7•II-7, and d) a mixture of II-1•II-1, (+)-II-5•(-)-II-5, and II-7•II-7.

\subsubsection{Selective Dissociation of One Member of a Self-Sorted Mixture.}

The heterodimerization of II-1 and II-2 revealed an important property of such system when considered from a different viewpoint. It enables a useful type of stimuli responsiveness that will be particularly useful in the engineering of complex and functional chemical systems. Consider, for example, a system comprising four members $(\mathrm{A}-\mathrm{D})$. Of the 10 homodimeric and heterodimeric species $(\mathrm{AA}, \mathrm{BB}, \mathrm{CC}$, $\mathrm{DD}, \mathrm{AB}, \mathrm{AC}, \mathrm{AD}, \mathrm{BC}, \mathrm{BD}, \mathrm{CD})$ that would comprise an unbiased dynamic combinatorial library, a high-fidelity self-sorting system is only able to access the 
four homodimeric states (e.g., AA, BB, CC, DD). The ability to selectively access heterodimeric states (e.g., BD) by suitable chemical stimuli (e.g., addition of molecular clip D) would allow fine tuning of the topology of the interaction network that characterizes this four-component mixture. ${ }^{101}$ In this context, we examined the addition of a fourth component to pre-existing three-component self-sorting systems. As expected, the addition of II-1•II-1 to a self-sorting mixture comprising II-2•II-2, $(+)-\mathbf{I I}-\mathbf{5} \bullet(-)-\mathbf{I I}-\mathbf{5}$, and $\mathbf{I I}-\mathbf{7} \cdot \mathbf{I I}-\mathbf{7}$ results in the selective heterodimerization of II-2•II-2 under the formation of II-1•II-2 (Figure II-13). We envision that the ability to selectively alter the interaction network of one component (or one subset of components) of a complex self-sorting mixture by addition of a new component will enable the construction of functional stimuli responsive systems.

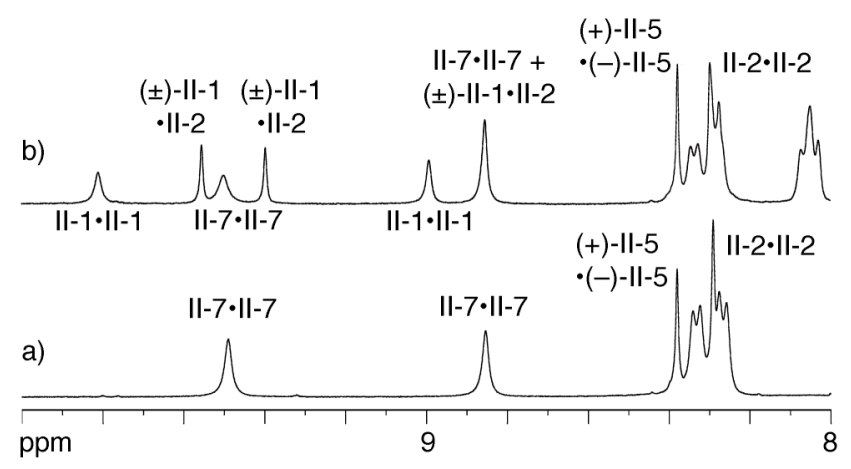

Figure II-13. ${ }^{1} \mathrm{H}$ NMR spectra (500 MHz, $\left.298 \mathrm{~K}\right)$ for: a) a mixture of II-2•II-2, (+)II-5•(-)-II-5, and II-7•II-7, and b) selective heterodimerization of II-2•II-2 under the formation of II-1•II-2 upon addition of II-1•II-1. 


\subsubsection{Steric Effects Influence the Thermodynamics and Kinetics of Dimerization.}

In the next phase of our search for self-sorting systems comprising larger numbers of molecular clips, we decided to introduce steric congestion onto the $o$ xylylene sidewalls. We hypothesized that increased steric interactions between two molecular clips would destabilize their homodimers more dramatically than their heterodimerics. This hypothesis is supported by simulations reported previously, ${ }^{93}$ that show that the system comprising one tight and one weak homodimer it is thermodynamically more favorable that the alternative arrangement featuring two heterodimers of modest strength. Therefore we anticipated that a mixture of two homodimers - one sterically hindered and one unsubstituted - might constitute a selfsorting system. We choose compound II-7 as the base system - because it forms a tight dimer and we thought it would be synthetically easier to modify - and gradually increased the number of substituents in order to test the influence of steric congestion on dimeric aggregation.

Accordingly, we prepared tetramethyl benzanilide II-8 and tetramethyl pivalanilide II-9. When a $2.5 \mathrm{mM}$ solution of $\mathbf{I I - 8}$ in $\mathrm{CDCl}_{3}$ was examined by ${ }^{1} \mathrm{H}$ NMR spectroscopy at room temperature, we observed two sets of peaks. After warming the same solution gradually to $55^{\circ} \mathrm{C}$, we observed a decrease in the intensity of one set of peaks concomitant with an increase in the intensity of the other set (Figure II-15). An indistinguishable spectrum was obtained when this solution was cooled slowly to room temperature. A similar change in the system was observed when an $11 \mathrm{mM}$ solution of II-8•II-8 was diluted 100-fold to $0.11 \mathrm{mM}$ (Figure II-14). 
These observations suggest that II-8 exists in equilibrium with II-8•II-8 with slow exchange kinetics relative to the chemical shift time scale at room temperature. Apparently, steric interactions between the methyl substituents on the aromatic walls of II-8-II-8 thermodynamically destabilize the dimer. Interestingly, however, the kinetics of exchange (e.g. $\mathrm{k}_{\text {off }}$ ) remain slow (as observed for II-7•II-7) even as $\mathrm{K}_{\mathrm{a}}$ is decreased. Conveniently, the observed slow exchange between monomer and dimer on the chemical shift time scale allows us to follow this equilibrium by ${ }^{1} \mathrm{H}$ NMR spectroscopy and calculate the association constant by measuring the integrals for monomer versus dimer at various concentrations $\left(\mathrm{K}_{\mathrm{s}}=2900 \pm 600 \mathrm{~mol}^{-1}\right)$. We incorporated additional steric congestion in II-9 in the form of $t-\mathrm{Bu}(\mathrm{C}=\mathrm{O}) \mathrm{NH}-\mathrm{H}-$ bonding arms (Figure II-18a). In this case, chemical shift of the aromatic as well as the amide protons do not change in the dilution experiment which suggests that II-9 exists as a monomer (Figure II-16). Interestingly, when we constructed an equimolar binary mixture of II-9 and II-9, an NMR spectrum was a superposition of the spectra of the components (Figure II-17). This result indicates that presence of severe steric congestion around the cleft of the hypothetical homodimer II-9-II-9 and heterodimer $( \pm)-\mathbf{I I}-8 \cdot I I-9$ dictates that compound II-9 must remain monomeric and form selfsorting mixture with II-8. 


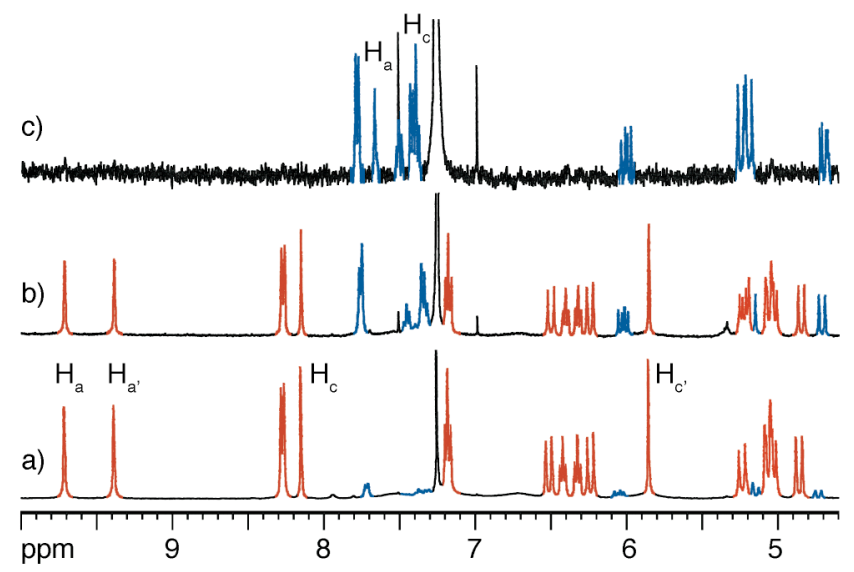

Figure II-14. Portion of the ${ }^{1} \mathrm{H}$ NMR spectra recorded for $9 \cdot 9\left(400 \mathrm{MHz}, \mathrm{CDCl}_{3}\right.$, RT) upon dilution: a) $11 \mathrm{mM}$, b) $1.1 \mathrm{mM}$, and c) $0.11 \mathrm{mM}$. Resonances are color coded as follows: dimeric II-8•II-8, red; and monomeric II-8, blue.

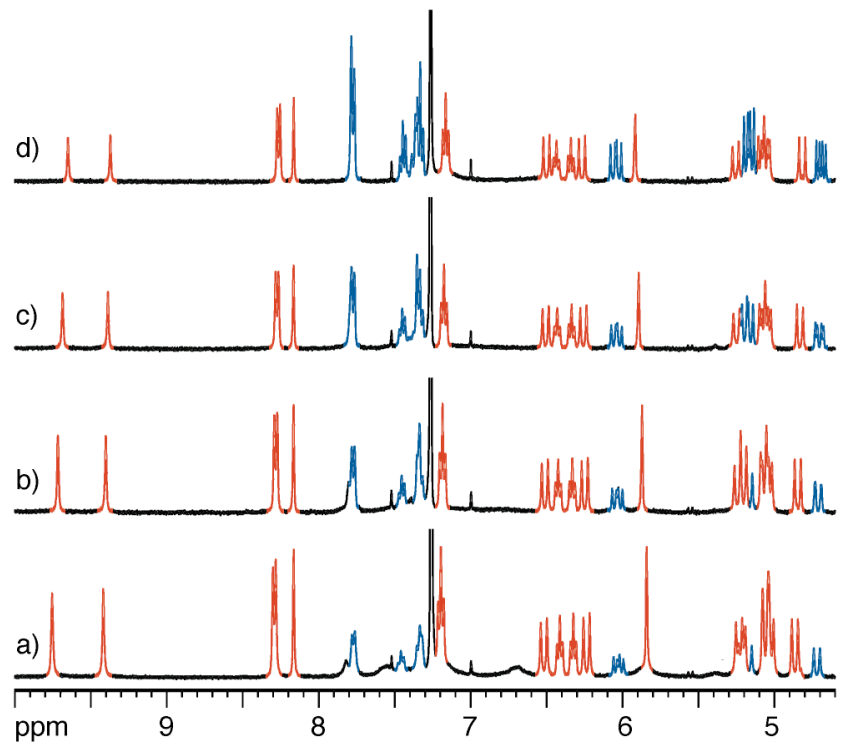

Figure II-15. Portion of the variable temperature ${ }^{1} \mathrm{H}$ NMR spectrum recorded for II8・II-8 (400 MHz, $\left.2.5 \mathrm{mM}, \mathrm{CDCl}_{3}, \mathrm{RT}\right)$ : a) $18^{\circ} \mathrm{C}$, b) $31^{\circ} \mathrm{C}$, c) $41^{\circ} \mathrm{C}$, and d) $55^{\circ} \mathrm{C}$. Resonances are color coded as follows: dimeric II-8•II-8, red; and monomeric II-8, blue. 


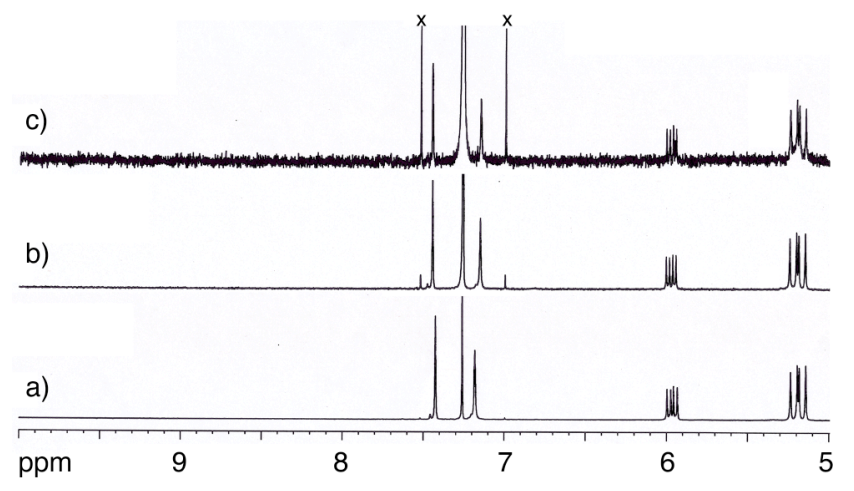

Figure II-16. Portion of the variable concentration ${ }^{1} \mathrm{H}$ NMR spectrum recorded for 9 (400 MHz, $\mathrm{CDCl}_{3}, \mathrm{RT}$ ): a) $10 \mathrm{mM}$, b) $1.0 \mathrm{mM}$, and c) $0.10 \mathrm{mM}$.

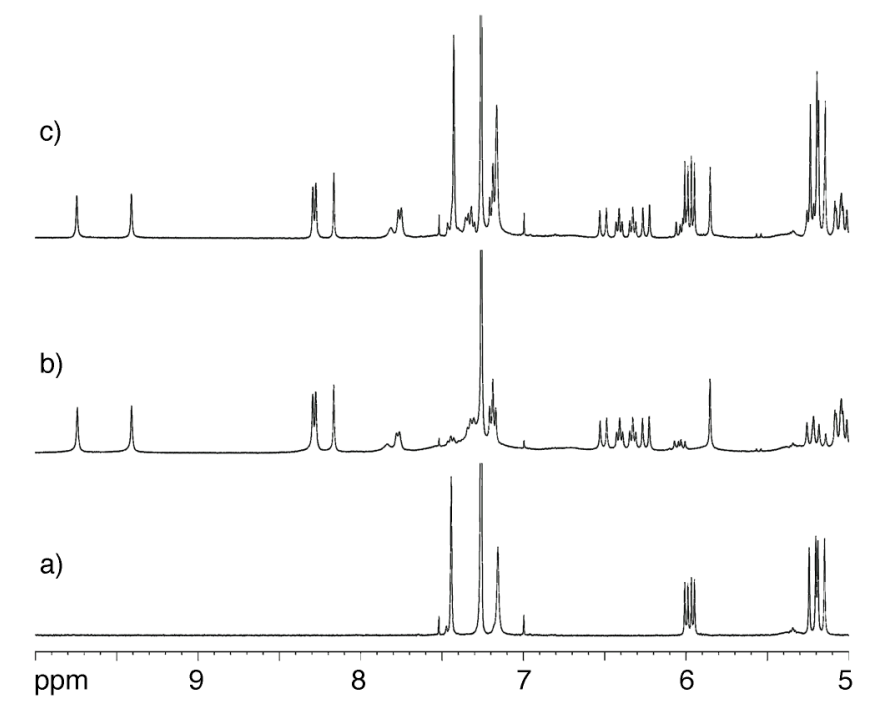

Figure II-17. ${ }^{1} \mathrm{H}$ NMR spectra (400 MHz, $298 \mathrm{~K}$ ) for: a) $\mathbf{I I - 9 ,}$, b) $\mathbf{I I - 8} \cdot \mathbf{I I - 8}$ in equilibrium with II-8, and c) a mixture of $\mathbf{I I - 8} \cdot \mathbf{I I - 8}$ in equilibrium with $\mathbf{I I - 8}$, and $\mathbf{I I - 9}$. 


\subsubsection{The Effect of Antiparallel Orientation of H-bonding Sidearms on Dimerization.}

As described earlier, the relative orientation of $\mathrm{H}$-bonding sidearms in $C_{\mathrm{s}^{-}}$or $C_{2}$-symmetrical molecular (e.g. parallel in II-1 and II-2 versus anti-parallel in ( \pm )-II4a and ( \pm -II-5) endow these molecular clips with efficient self- versus non-self dicrimination abilities. Accordingly, we prepared $( \pm)$-II-10 and ( \pm )-II-11 which are the $C_{2}$-symmetric diastereomers of $C_{\mathrm{s}}$-symmetric II-7 and II-8. Interestingly, although not unexpectedly, we did not find any evidence of discrete dimer formation in the ${ }^{1} \mathrm{H}$ NMR spectra of $( \pm)$-II-10 and $( \pm)$-II-11. In the molecular structures of $( \pm)$ II-10 and ( \pm )-II-11 the H-bonding pendant arms are displayed in divergent directions on the tips of the aromatic rings and therefore block the face of the cleft and create steric hindrance to dimerization by association with a second molecular clip. Molecular clips ( \pm )-II-10 and ( \pm )-II-11 do not form well-defined discrete dimers like those formed by II-7 or II-8. Rather, we observe broadening and upfield shifting in their ${ }^{1} \mathrm{H}$ NMR spectra which indicates that $( \pm)-\mathbf{I I}-10$ and $( \pm)$-II-11 form poorly structured oligomeric species in solution (Figure II-19). The solid state behavior is similarly affected; unlike II-7 that exists as dimer, compound ( \pm )-II-11 remain monomeric in the crystal (Figure II-18b). These results - which show that both $\pi-\pi$ and $\mathrm{H}$-bonds required to form discrete dimeric aggregates - further validate the use of molecular clips as a platform for the construction complex self-sorting systems. For example, the transition from monomer to discrete dimer to poorly defined oligomeric states can be programmed into the system by the identity and spatial orientation of the substituents on the $o$-xylylene sidewalls of the constituent molecular clips. 
a)

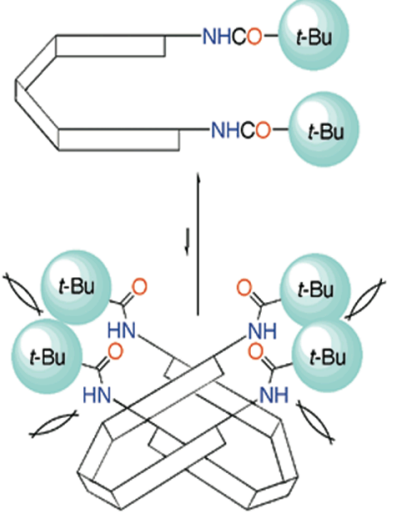

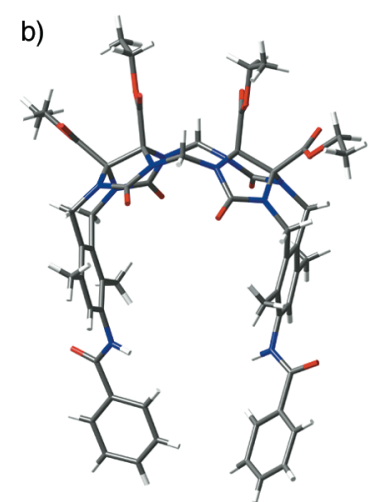

Figure II-18. Molecular clips that remain monomeric in solution: a) Steric bulk prevents dimeric aggregation of II-9, and b) Crystal structure of ( \pm )-II-11 showing the display of H-bonding groups in front of the cleft which leads to steric hindrance to dimerization.

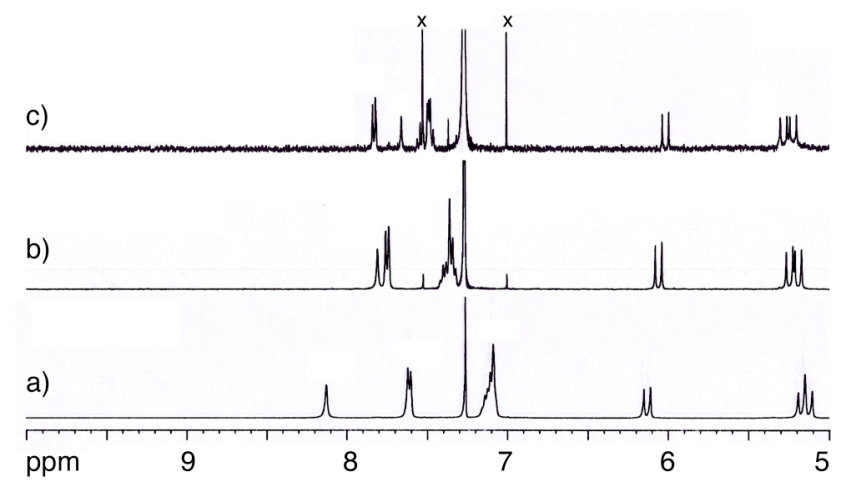

Figure II-19. Portion of the variable concentration ${ }^{1} \mathrm{H}$ NMR spectrum recorded for ( \pm )-II-11 (400 MHz, $\left.\mathrm{CDCl}_{3}, \mathrm{RT}\right):$ a) $10 \mathrm{mM}$, b) $1.0 \mathrm{mM}$, and c) $0.10 \mathrm{mM}$. 


\subsubsection{Effect of Additional H-bonding Groups on the Ability of Molecular Clips to Undergo Self-Assembly and Self-Sorting.}

After exploring the influence of geometry, sterics, and chirality on the ability of molecular clips to undergo self-assembly and self-sorting we decided to investigate the influence of the number and pattern of H-bonding donors and acceptors in the pendant arm on the fidelity of self-sorting. We synthesized II-3 which contains additional $\mathrm{NH}(\mathrm{CO}) \mathrm{CH}_{3} \mathrm{H}$-bonding groups in the para-position of the pendant aromatic rings. We thought that $\mathbf{I I - 3}$ might form a very robust aggregate due to an increased number of H-bonding interactions upon dimerization. We further predicted that II-3 might undergo self-sorting with II-1 or II-2 based on differences in the number of H-bonds in the aggregate (Figure II-20a, II-20b). When II-3 was dissolved in $\mathrm{CDCl}_{3}$ we observed upfield shifting, severe broadening and multiple resonances for some protons which indicates the formation of multiple ill defined species in Upon further examination of a CPK model of II-3, we realized that the flexibility of the pendant arm of II-3 increases as the arm gets longer. Interestingly, examination of the X-ray crystal structures of II-1 and II-2 show that the two internal amide protons remain free in the dimeric aggregate. Upon careful inspection, we could conclude that these amide protons are actually involved in weak electrostatic interactions with the ureidyl $\mathrm{C}=\mathrm{O}$ group and also they are not accessible to any external H-bond acceptors because they are buried inside the molecular architecture. Due to this lack of rigidity, unsatisfied H-bond donors in the amide group farthest 
from the clip can interact with unsatisfied H-bond acceptors on other molecules of II$\mathbf{3}$ or assemblies of II-3 resulting in uncontrolled higher order aggregation.
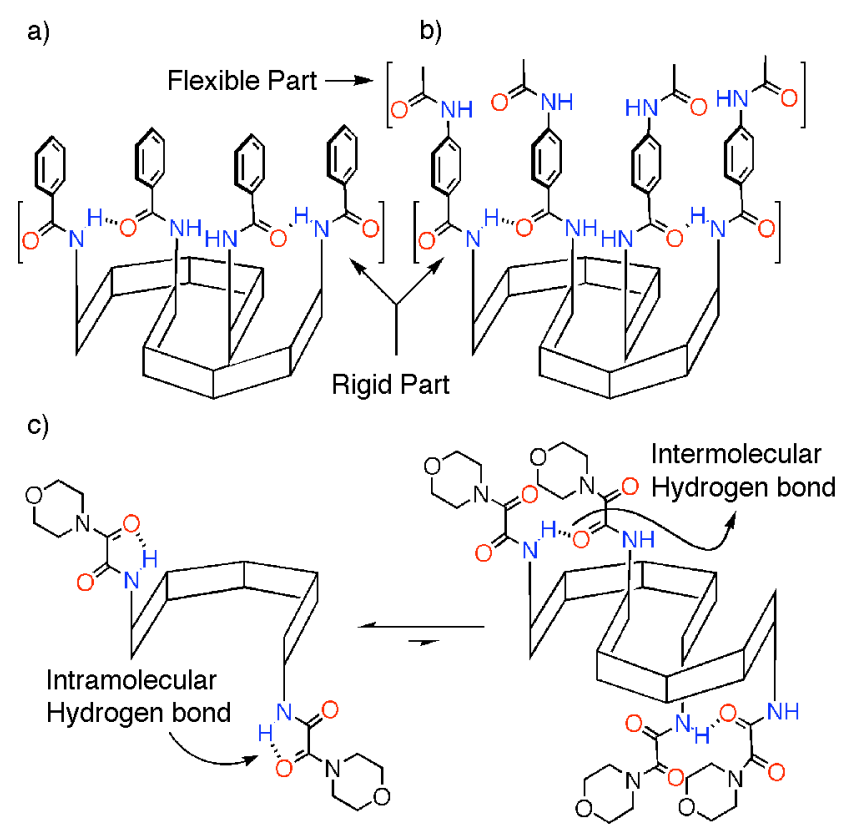

Figure II-20. Schematic representation of the aggregates formed by a) II-1-II-1, b) II-3•II-3 and c) ( \pm )-II-6.

Compounds II-2 and ( \pm )-II-5 contain H-bond donor-acceptor-donor arrangement in the ureidyl group. We hypothesized that a molecular clip with a specific donor-acceptor arrangement in the pendant H-bonding arms might undergo self-sorting with another clip that possessed a different donor-acceptor arrangement. In order to test that hypothesis, we synthesized compound ( \pm )-II-6 containing Hbonding groups having a donor-acceptor-acceptor pattern. When dissolved in $\mathrm{CDCl}_{3}$, we observed an upfield shift of the proton attached to the $o$-xylylene sidewall of ( \pm )-II-6 indicating that $( \pm)$-II-6 forms an aggregate in solution. Unlike (+)-II4a・(-)-II-4a and (+)-II-5•(-)-II-5, we did not observe any doubling of resonances 
due to aggregation as the molecule undergoes fast exchange on the chemical shift time scale. Interestingly, when a solution of ( \pm )-II-6 was diluted, we observed a down field shift of the amide protons (Figure II-21). Usually the amide protons move upfield in dilution experiments as the H-bonds are disrupted with increasing dilution. This result indicates the presence of a two strong intramolecular H-bond in monomer $( \pm)-$ II-6 which are sacrificed to form two weak intermolecular H-bonds and $\pi-\pi$ interactions during dimerization. Accordingly, compound ( \pm )-II-6 form a weak dimer $\left(\mathrm{K}_{\mathrm{s}}=17.5 \pm 2.2 \mathrm{~mol}^{-1}\right)$ in $\mathrm{CDCl}_{3}$ solution (Figure II-20c). When we mixed ( \pm )II-6 with an equimolar amount of ( \pm )-II-5, we mainly observed the separate coexistence of $( \pm)-\mathbf{I I}-\mathbf{6}$ and $(+)-\mathbf{I I}-5 \cdot(-)-\mathbf{I I}-5$ in the solution with very small amount of heteromeric assemblies (Figure II-22). This observation suggests that the tendency of molecular clips with common geometrical arrangement of their H-bonding arms toward formation of mixtures of homo- and heterodimers can be suppressed with concomitant enhancement of self-selectivity can be achieved by incorporating an electronic mismatch between their H-bonding arms. 


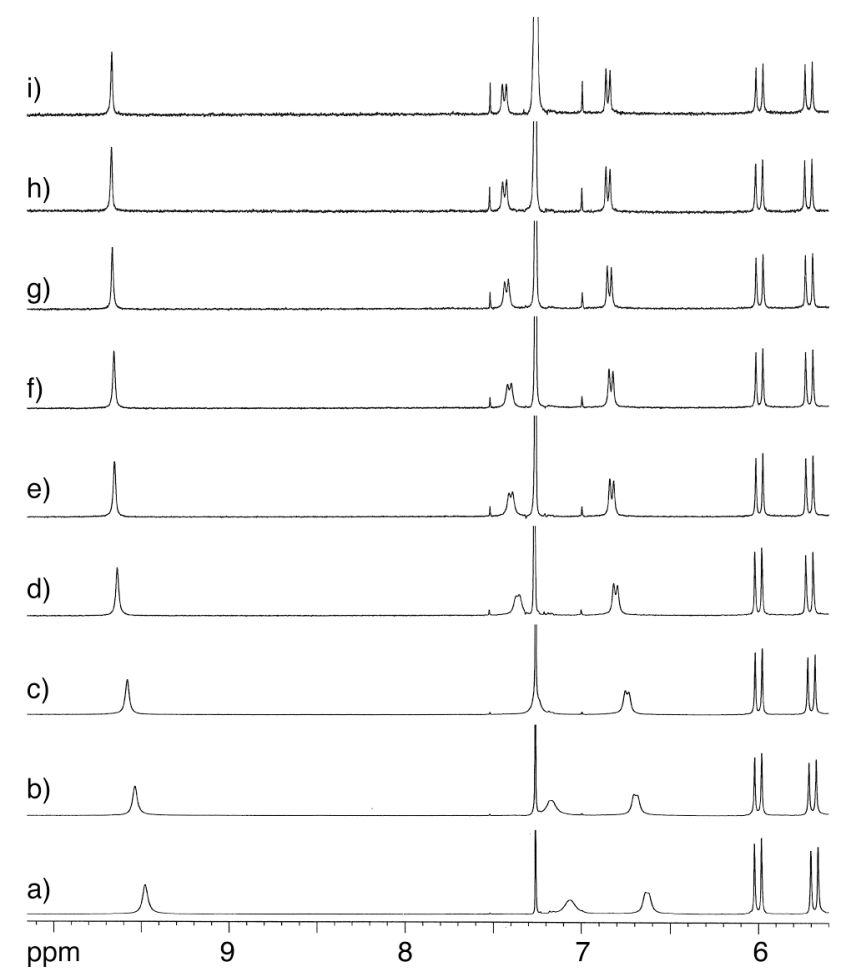

Figure II-21. Portion of the variable concentration ${ }^{1} \mathrm{H}$ NMR spectrum recorded for ( \pm )-II-6 (400 MHz, $\left.\mathrm{CDCl}_{3}, \mathrm{RT}\right)$ : a) $15.51 \mathrm{mM}$, b) $9.30 \mathrm{mM}$, and c) $5.58 \mathrm{mM}$, d) 2.23 $\mathrm{mM}$, e) $1.11 \mathrm{mM}$, f) $0.89 \mathrm{mM}, \mathrm{g}) 0.55 \mathrm{mM}, \mathrm{h}) 0.35 \mathrm{mM}$, i) $0.27 \mathrm{mM}$.

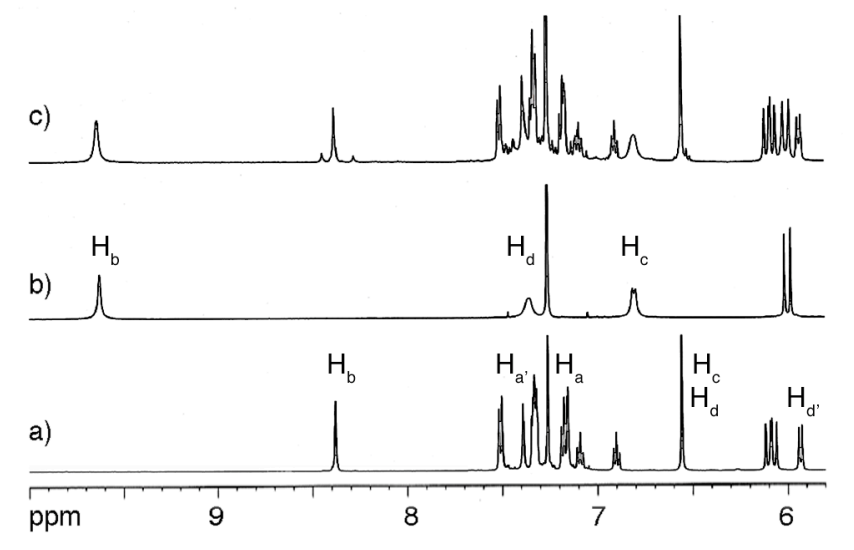

Figure II-22. ${ }^{1} \mathrm{H}$ NMR $\left(2 \mathrm{mM}, 500 \mathrm{MHz}, \mathrm{CDCl}_{3}, \mathrm{RT}\right)$ spectrum recorded for: a) (+)II-5•(-)-II-5, b) ( \pm )-II-6, and c) a mixture of (+)-II-5•(-)- II-5, and ( \pm )-II-6. 


\subsubsection{Four-Component Self-Sorting Systems.}

If we consider that only monomeric and dimeric aggregates can exist in the solution then a mixture of four molecular clips (A, B, C, D) can give rise to fourteen different species (A, B, C, D, $\mathrm{A}_{2}, \mathrm{~B}_{2}, \mathrm{C}_{2}, \mathrm{D}_{2}, \mathrm{AB}, \mathrm{AC}, \mathrm{AD}, \mathrm{BC}, \mathrm{BD}, \mathrm{CD}$ ) in the solution. In our quest for a four component self-sorting system we screened a large number of different combinations guided by the principles discussed above. Initially, we examined an equimolar mixture of $\mathbf{I I}-\mathbf{2},( \pm)-\mathbf{I I}-\mathbf{5}$, and $\mathbf{I I - 8}$ and observed a selfsorted mixture by ${ }^{1} \mathrm{H}$ NMR spectroscopy due to the disparate spatial orientation of their H-bonding arm. Based on the fact that II-8 and II-9 constitute a self-sorting system (vide supra) we decided to prepare a mixture comprising molecular clips II-2, ( \pm )-II-5, II-8, and II-9. Remarkably, this set of four molecular clips exists in a selfsorted state (Figure II-23) comprising five different species - II-2•II-2, (+)-II-5•(-)II-5, II-8 in equilibrium with II-8•II-8, and monomeric II-9 - out of fourteen possibilities. 


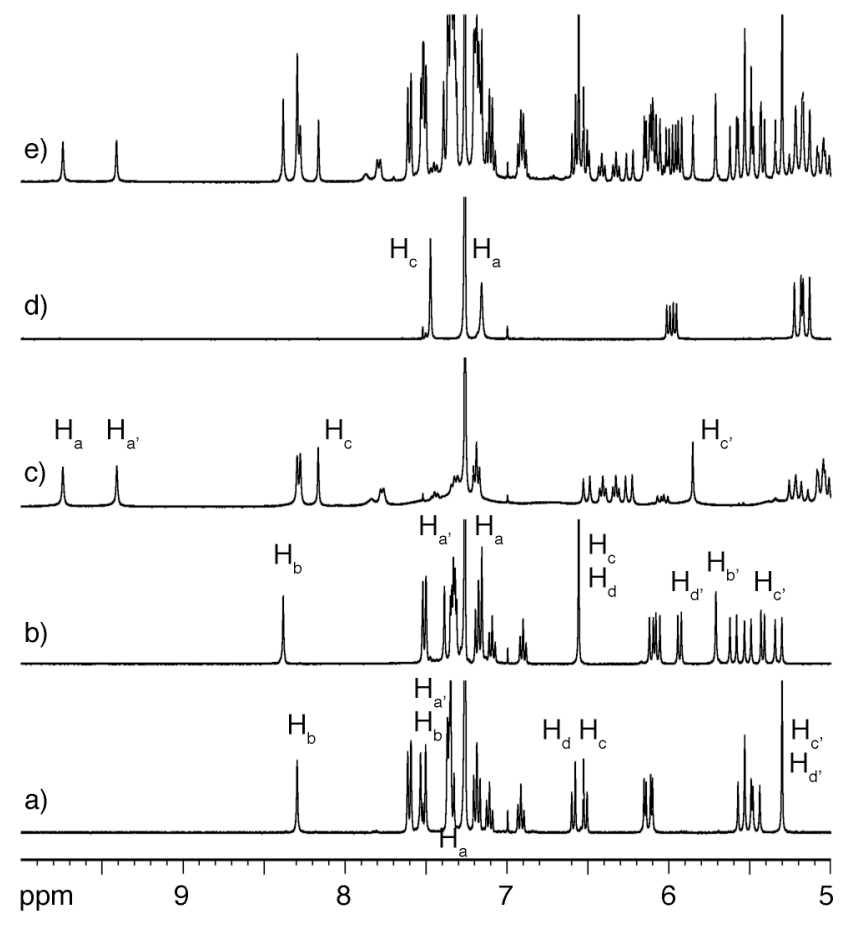

Figure II-17. ${ }^{1} \mathrm{H}$ NMR spectra (400 MHz, $\left.298 \mathrm{~K}\right)$ for: a) II-2•II-2, b) (+)-II-5•(-)II-5, c) II-8 in equilibrium with II-8•II-8, d) II-9, and e) a mixture of II-2•II-2, (+)II-5•(-)-II-5, II-8 in equilibrium with II-8•II-8, and II-9.

\subsection{Conclusion.}

In summary, we presented the synthesis of methylene-bridged glycoluril dimers II-1 - II-11, which undergo strong dimerization in $\mathrm{CDCl}_{3}$ due to a combination of $\mathrm{H}$-bonding and $\pi-\pi$ interactions. The structure of these dimeric aggregates changes as the position and relative orientation of the two H-bonding groups move around the $o$-xylylene rings. For example, II-1·II-1 and II-2·II-2 form homodimers and (+)-II-4a·(-)-II-4a and (+)-II-5•(-)-II-5 undergo heterochiral recognition, both with an in register type geometry. When H-bonding groups were 
moved to the tips of the aromatic rings (e.g., II-7·II-7 and II-8·II-8), a skewed geometry was observed that maximizes H-bonding and $\pi-\pi$ interactions. Steric bulkiness around the cleft of these molecular clips (e.g., II-8 and II-9) leads to reduced values of $K_{\mathrm{a}}$, but slow exchange kinetics relative to the ${ }^{1} \mathrm{H}$ NMR time scale are maintained. The high levels of self-selectivity of these molecular clips allowed us to prepare three-component (II-2, ( \pm )-II-5, and II-7) and even a four-component selfsorted system (II-2, ( \pm )-II-5, II-8, and II-9).

The implications of this research go beyond the system-specific considerations described above. The availability of a series of robust, easily functionalized, and orthogonal H-bonding modules for assembly in nonpolar solvents such as $\mathrm{CDCl}_{3}$ can be utilized for numerous applications. For example, the patterning of surfaces using two orthogonal recognition units has recently been demonstrated; additional levels of patterning could be added along with stimuli control based on molecular clips reported herein. Such modules also enable the noncovalent derivatization of polymer backbones in solution to optimize their properties for specific applications (e.g., light emitting diodes, drug delivery, tissue engineering). Although the molecular clips described herein assemble in nonpolar solvents, watersoluble versions of these compounds also assemble in water. When such watersoluble molecular clips also exhibit self-sorting, it would be possible to use them as tags to promote dimerization of appended (bio)molecules within complex cellular environments.

Perhaps most significantly, the development of dynamic combinatorial chemistry and self-sorting systems has helped stimulate the development of systems 
chemistry. In unbiased dynamic combinatorial libraries all constituent states are equally populated and application of a chemical stimulus leads to enhanced formation of one or more members of the library due to favorable non-covalent interactions. Similar to natural systems whose non-covalent interaction networks are tightly controlled self-sorting systems typically comprise only a small fraction of the conceivable non-covalent aggregates. In this chapter, we demonstrated that the addition of a new molecular clip (e.g., II-1) to a self-sorting mixture (e.g., II-2-II-2, $(+)-I I-5 \cdot(-)-I I-5$, and II-7-II-7) results in the selective heterodimerization of only one member of the system. By extension, it should be possible to prepare larger systems comprising sets of molecular clips with common spatial distribution of their $\mathrm{H}-$ bonding arms (e.g., II-1 - II-3, II-4 - II-6, and III-7 - II-9) that display self-sorting between sets but not within sets. The development of stimuli responsive versions of such self-sorting systems that result in new connection between sets promises the development of complex systems that exhibit behaviors typically reserved for natural systems.

\subsection{Experimental.}

\subsubsection{General Experimental.}

Starting materials were purchased from Alfa-Aesar, Acros, and Aldrich and were used without further purification. Compounds II-1, II-2, $( \pm)-\mathbf{I I}-4,( \pm)-\mathbf{I I}-5$, II12 - II-15, II-19 and 4-morpholineglyoxyloyl chloride were prepared according to the literature procedures. Melting points were measured on a Meltemp apparatus in 
open capillary tubes and are uncorrected. NMR spectra were measured on Bruker AM-400, DRX-400, and DMX-500 instruments operating at 400 or $500 \mathrm{MHz}$ for ${ }^{1} \mathrm{H}$ and 100 or $125 \mathrm{MHz}$ for ${ }^{13} \mathrm{C}$. Mass spectrometry was performed using a VG $7070 \mathrm{E}$ magnetic sector instrument by electron impact (EI) or by fast atom bombardment (FAB) using the indicated matrix. The matrix "magic bullet" is a 5:1 (w:w) mixture of dithiothreitol:dithioerythritol.

\subsubsection{Synthetic Procedures and Characterization.}

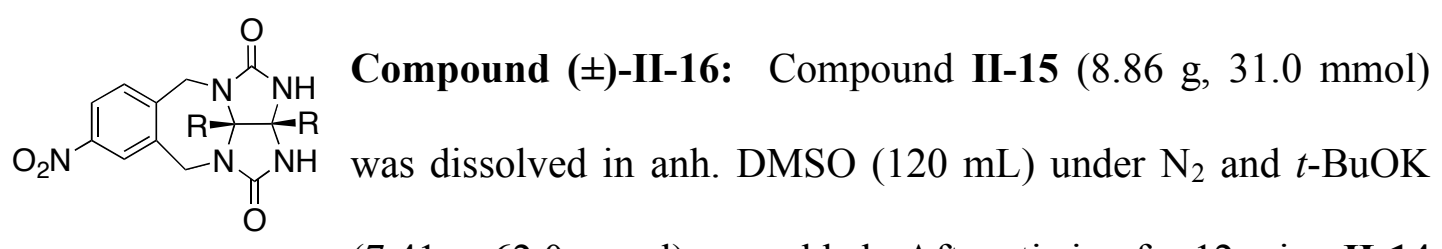

$( \pm)-16 \mathrm{R}=\mathrm{CO}_{2} \mathrm{Et} \quad(7.41 \mathrm{~g}, 62.0 \mathrm{mmol})$ was added. After stirring for $12 \mathrm{~min} ., \mathbf{I I}-14$ $(2.10 \mathrm{~g}, 6.20 \mathrm{mmol})$ was added in one portion and stirring was continued for $2 \mathrm{~h}$. The reaction mixture was poured into $0.1 \mathrm{~N} \mathrm{HCl}(1 \mathrm{~L})$ and extracted with EtOAc $(3 \mathrm{x}$ $1000 \mathrm{~mL})$. The extracts were washed with brine $(2 \times 500 \mathrm{~mL})$, dried over anh. $\mathrm{MgSO}_{4}$ and concentrated. Flash chromatography $\left(\mathrm{SiO}_{2}, \mathrm{CHCl}_{3} / \mathrm{MeOH} 25: 1\right)$ gave impure $( \pm)$-II-16 as a yellow solid. The impure solid was washed with EtOAc (1 $\mathrm{mL}$ ), centrifuged, the supernatant decanted and the residue dried at high vacuum to obtain pure $( \pm)$-II-16 as a white solid (650 mg, $1.50 \mathrm{mmol}, 22 \%)$. M.p. $125-126{ }^{\circ} \mathrm{C}$. TLC $\left(\mathrm{CHCl}_{3} / \mathrm{MeOH} 25: 1\right) R_{\mathrm{f}}$ 0.14. IR $\left(\mathrm{KBr}, \mathrm{cm}^{-1}\right): 3422 \mathrm{~m}, 2985 \mathrm{w}, 1717 \mathrm{~s}, 1527 \mathrm{~m}$, 1463m, 1350m, 1271m. ${ }^{1} \mathrm{H}$ NMR (400 MHz, $\left.\mathrm{CDCl}_{3}\right): 8.19$ (d, $\left.J=2.2,1 \mathrm{H}\right), 8.05$ (dd, $J=2.2, J=8.2,1 \mathrm{H}), 7.51(\mathrm{~d}, J=8.2,1 \mathrm{H}), 6.30(\mathrm{~s}, 1 \mathrm{H}), 6.11(\mathrm{~s}, 1 \mathrm{H}), 4.93(\mathrm{~d}, J=$ $16.1,1 \mathrm{H}), 4.92(\mathrm{~d}, J=16.1,1 \mathrm{H}), 4.47(\mathrm{~d}, J=16.1,1 \mathrm{H}), 4.46(\mathrm{~d}, J=16.1,1 \mathrm{H}), 4.32$ 
(q, $J=7.1,2 \mathrm{H}), 4.24(\mathrm{q}, J=7.1,2 \mathrm{H}), 1.32(\mathrm{t}, J=7.1,3 \mathrm{H}), 1.27(\mathrm{t}, J=7.1,3 \mathrm{H}) .{ }^{13} \mathrm{C}$ NMR (100 MHz, $\left.\mathrm{CDCl}_{3}\right): \delta 166.0,165.7,157.2,157.0,148.0,143.6,138.5,131.2$, $124.8,123.8,83.1,74.0,64.2,63.9,44.6,14.4,14.2$ (only 17 of the 18 expected resonances were observed). MS (FAB, magic bullet): $m / z 434\left(100,[\mathrm{M}+\mathrm{H}]^{+}\right)$. HRMS (FAB, magic bullet, PEG): $m / z 434.1303\left([\mathrm{M}+\mathrm{H}]^{+}, \mathrm{C}_{18} \mathrm{H}_{20} \mathrm{~N}_{5} \mathrm{O}_{8}\right.$, calcd 434.1312).
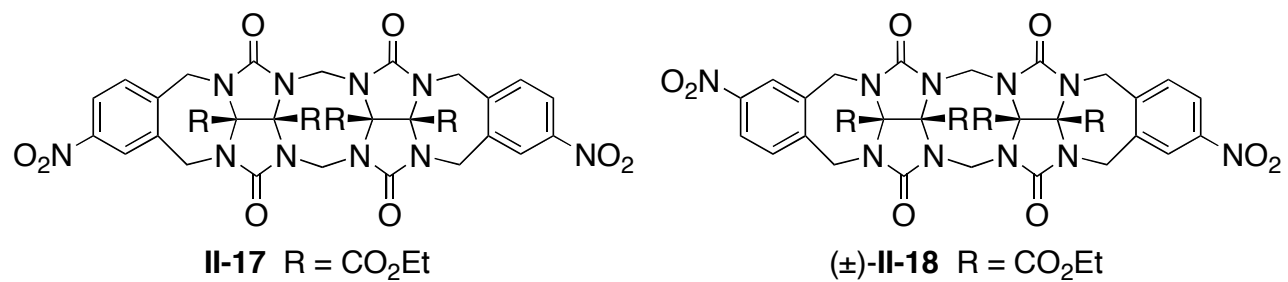

Compound II-17 and ( \pm )-II-18: A mixture of PTSA (3.51g, $18.4 \mathrm{mmol})$ in $\mathrm{ClCH}_{2} \mathrm{CH}_{2} \mathrm{Cl}(100 \mathrm{~mL})$ was heated under $\mathrm{N}_{2}$ at reflux for 30 min. under an addition

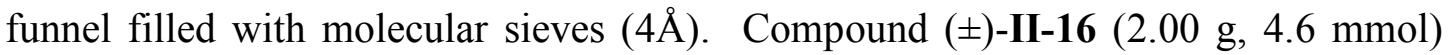
and paraformaldehyde (414 mg, $13.8 \mathrm{mmol}$ ) were added and reflux was continued for 4 days. The reaction mixture was diluted with EtOAc $(1000 \mathrm{~mL})$, washed with sat. aq. $\mathrm{Na}_{2} \mathrm{CO}_{3}$, dried over anh. $\mathrm{MgSO}_{4}$, and concentrated. Flash chromatography $\left(\mathrm{SiO}_{2}\right.$, $\mathrm{CHCl}_{3} / \mathrm{CH}_{3} \mathrm{CN}$ 3:1 and 1:1) gave impure ( \pm )-II-18 (447 mg, $\left.0.502 \mathrm{mmol}, 22 \%\right)$ and II-17 (566 mg, $0.636 \mathrm{mmol}, 28 \%)$, which were washed with EtOAc $(1 \mathrm{~mL})$, centrifuged, the supernatant decanted, and the residue dried under high vacuum, yielding product $( \pm)$-II-18 (405 $\mathrm{mg}, 0.455 \mathrm{mmol}, 20 \%)$ and $\mathbf{I I - 1 7}(479 \mathrm{mg}, 0.538$ mmol, 23\%) as white solids. Compound ( \pm )-II-18: M.p. $>300{ }^{\circ} \mathrm{C}$ (dec.). TLC $\left(\mathrm{CHCl}_{3} / \mathrm{MeOH} 25: 1\right) R_{\mathrm{f}} 0.12$. IR (KBr, $\left.\mathrm{cm}^{-1}\right): 2984 \mathrm{w}, 1749 \mathrm{~s}, 1528 \mathrm{~m}, 1453 \mathrm{~s}, 1349 \mathrm{~m}$, 
1254s, $1019 \mathrm{~m}, 911 \mathrm{~m} .{ }^{1} \mathrm{H}$ NMR (400 MHz, DMSO- $\left.d_{6}\right): 8.15$ (s, 2H), $8.02(\mathrm{~d}, J=$ $8.2,2 \mathrm{H}), 7.56(\mathrm{~d}, J=8.2,2 \mathrm{H}), 5.79(\mathrm{~d}, J=16.2,2 \mathrm{H}), 4.92(\mathrm{~d}, J=16.2,2 \mathrm{H}), 4.83(\mathrm{~d}, J$ $=16.2,2 \mathrm{H}), 4.66(\mathrm{~d}, J=16.2,2 \mathrm{H}), 4.62(\mathrm{~d}, J=16.2,2 \mathrm{H}), 4.53(\mathrm{~d}, J=16.2,2 \mathrm{H})$, 4.30-4.20 (m, 8H), 1.30-1.15 (m, 12H). ${ }^{13} \mathrm{C}$ NMR (100 MHz, DMSO- $\left.d_{6}\right): \delta$ 164.6, $163.8,153.9,146.9,143.9,138.5,130.9,123.9,123.2,79.8,78.3,64.5,64.0,47.4$, 44.0, 43.8, 13.6, 13.5 (only 18 of the 19 expected resonances were observed). MS (FAB, magic bullet): $m / z 891\left(100,[\mathrm{M}+\mathrm{H}]^{+}\right)$. HR-MS (FAB, magic bullet, CsI): $m / z$ $1023.1505\left([\mathrm{M}+\mathrm{Cs}]^{+}, \mathrm{C}_{38} \mathrm{H}_{38} \mathrm{~N}_{10} \mathrm{O}_{16} \mathrm{Cs}\right.$, calcd 1023.1522). Compound II-17: M.p. $>280{ }^{\circ} \mathrm{C}$ (dec.). $\mathrm{TLC}\left(\mathrm{CHCl}_{3} / \mathrm{MeOH} 25: 1\right) R_{\mathrm{f}} 0.07$. IR $\left(\mathrm{KBr}, \mathrm{cm}^{-1}\right): 2984 \mathrm{w}, 1748 \mathrm{~s}$, 1528m, 1455s, 1349m, 1255s, 1019m, 912m. ${ }^{1} \mathrm{H}$ NMR (400 MHz, DMSO-d 6 ): 8.16 (s, 2H), $8.02(\mathrm{~d}, J=8.0,2 \mathrm{H}), 7.56(\mathrm{~d}, J=8.0,2 \mathrm{H}), 5.79(\mathrm{~d}, J=16.1,1 \mathrm{H}), 5.78(\mathrm{~d}, J=$ 16.1, 1H), $4.92(\mathrm{~d}, J=16.1,2 \mathrm{H}), 4.83(\mathrm{~d}, J=16.1,2 \mathrm{H}), 4.66(\mathrm{~d}, J=16.1,2 \mathrm{H}), 4.62$ (d, $J=16.1,2 \mathrm{H}), 4.53(\mathrm{~d}, J=16.1,1 \mathrm{H}), 4.52(\mathrm{~d}, J=16.1,1 \mathrm{H}), 4.30-4.20(\mathrm{~m}, 8 \mathrm{H})$, 1.30-1.15 (m, 12H). ${ }^{13} \mathrm{C}$ NMR (100 MHz, DMSO- $\left.d_{6}\right): \delta 164.6,163.8,154.0,153.8$, $146.9,143.9,138.5,130.7,124.0,123.1,79.8,78.3,64.5,64.0,47.5,43.9,43.8,13.6$, 13.5 (only 19 of the 20 expected resonances were observed). MS (FAB, magic bullet): $m / z 891\left(100,[\mathrm{M}+\mathrm{H}]^{+}\right)$. HR-MS (FAB, magic bullet): $m / z 1023.1483([\mathrm{M}+$ $\mathrm{Cs}]^{+}, \mathrm{C}_{38} \mathrm{H}_{38} \mathrm{~N}_{10} \mathrm{O}_{16} \mathrm{Cs}$, calcd 1023.1522).

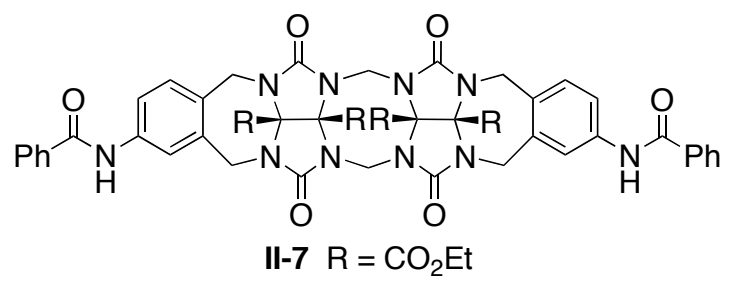

Compound II-7: A mixture of compound II-17 (267 mg, $0.30 \mathrm{mmol})$ and $10 \% \mathrm{Pd} / \mathrm{C}(200 \mathrm{mg})$ in anh. DMF (20

$\mathrm{mL})$ was stirred under $\mathrm{H}_{2}(10 \mathrm{psi})$ at $\mathrm{RT}$ for $6 \mathrm{~h}$ in a Schlenk apparatus. The reaction 
mixture was filtered under Ar and concentrated under high vacuum at RT to obtain a highly air and moisture sensitive diamine as a white solid. The crude solid was dissolved in a mixture of anh. degassed $\mathrm{CH}_{2} \mathrm{Cl}_{2}(10 \mathrm{~mL})$ and distilled $\mathrm{NEt}_{3}(1 \mathrm{~mL})$ under Ar. This solution was added to a solution of benzoyl chloride (168 mg, 1.20 mmol) in anh. degassed $\mathrm{CH}_{2} \mathrm{Cl}_{2}(5 \mathrm{~mL})$ at $-78{ }^{\circ} \mathrm{C}$. After $15 \mathrm{~min}$., the cooling bath was removed and stirring was continued at room temperature for $8 \mathrm{~h}$ under Ar. The reaction mixture was diluted with $\mathrm{CHCl}_{3}(200 \mathrm{~mL})$, washed with sat. aq. $\mathrm{NaHCO}_{3}$, dried over anh. $\mathrm{MgSO}_{4}$, and concentrated. Flash chromatography $\left(\mathrm{SiO}_{2}\right.$, $\mathrm{CHCl}_{3} / \mathrm{MeOH}$ 20:1) gave impure II-7. The impure solid was washed with EtOAc (2 $\mathrm{mL}$ ), centrifuged, the supernatant decanted and the residue dried at high vacuum to obtain pure II-7 as a white solid (227 mg, $0.218 \mathrm{mmol}, 73 \%)$. M.p. $>319{ }^{\circ} \mathrm{C}(\mathrm{dec}$.). TLC $\left(\mathrm{CHCl}_{3} / \mathrm{MeOH} 10: 1\right) R_{\mathrm{f}}$ 0.44. IR (KBr, $\left.\mathrm{cm}^{-1}\right): 3435 \mathrm{~m}, 2983 \mathrm{w}, 1745 \mathrm{~s}, 1659 \mathrm{~m}$, 1596m, 1535m, 1456m, 1426m, 1254s, 1017m, 910m. ${ }^{1} \mathrm{H}$ NMR (400 MHz, DMSO$\left.d_{6}\right): 10.19(\mathrm{~s}, 2 \mathrm{H}), 7.88(\mathrm{~d}, J=7.4,4 \mathrm{H}), 7.68(\mathrm{~s}, 2 \mathrm{H}), 7.65-7.50(\mathrm{~m}, 4 \mathrm{H}), 7.50-7.40$ (m, 4H), $7.23(\mathrm{~d}, J=8.2,2 \mathrm{H}), 5.81(\mathrm{~d}, J=16.1,1 \mathrm{H}), 5.80(\mathrm{~d}, J=16.1,1 \mathrm{H}), 4.65-4.40$ (m, 10H), 4.30-4.10 (m, 8H), $1.24(\mathrm{t}, J=7.1,6 \mathrm{H}), 1.20(\mathrm{t}, J=7.1,6 \mathrm{H}) .{ }^{13} \mathrm{C}$ NMR (100 MHz, DMSO- $\left.d_{6}\right): \delta 165.4,165.1,164.1,154.1,154.0,138.7,136.5,134.7$, $131.5,131.2,129.9,128.3,127.6,121.3,119.3,80.0,78.5,64.3,63.8,47.3,44.9$, $44.2,13.6,13.5$ (only 24 of the 25 expected resonances were observed). MS (FAB, magic bullet): $m / z 1039\left(35,[\mathrm{M}+\mathrm{H}]^{+}\right), 105(100)$. HR-MS (FAB, magic bullet, CsI): $m / z 1171.2565\left([\mathrm{M}+\mathrm{Cs}]^{+}, \mathrm{C}_{52} \mathrm{H}_{50} \mathrm{~N}_{10} \mathrm{O}_{14} \mathrm{Cs}\right.$, calcd 1171.2562). 
${ }^{H}$

( \pm )-II-10 R $=\mathrm{CO}_{2} \mathrm{Et}$

Compound ( \pm -II-10: A mixture of compound $( \pm)-\mathbf{I I}-18 \quad(138 \mathrm{mg}, \quad 0.16$ $\mathrm{mmol})$ and $10 \% \mathrm{Pd} / \mathrm{C}(100 \mathrm{mg})$ in anh. DMF (15 mL) was stirred under $\mathrm{H}_{2}(10 \mathrm{psi})$ at $\mathrm{RT}$ for $6 \mathrm{~h}$ in a Schlenk apparatus. The reaction mixture was filtered under Ar and concentrated under high vacuum at RT to obtain a highly air and moisture sensitive diamine as a white solid. The crude solid was dissolved in a mixture of anh. degassed $\mathrm{CH}_{2} \mathrm{Cl}_{2}(5 \mathrm{~mL})$ and distilled $\mathrm{NEt}_{3}(1 \mathrm{~mL})$ under Ar. This solution was added to a solution of benzoyl chloride (46 mg, 0.33 mmol) in anh. degassed $\mathrm{CH}_{2} \mathrm{Cl}_{2}(5 \mathrm{~mL})$ at $-78{ }^{\circ} \mathrm{C}$. After 15 min., the cooling bath was removed and stirring was continued at room temperature for $8 \mathrm{~h}$ under Ar. The reaction mixture was diluted with $\mathrm{CHCl}_{3}(200 \mathrm{~mL})$, washed with sat. aq. $\mathrm{NaHCO}_{3}$, dried over anh. $\mathrm{MgSO}_{4}$, and concentrated. Flash chromatography $\left(\mathrm{SiO}_{2}\right.$, $\left.\mathrm{CHCl}_{3} / \mathrm{MeOH} 50: 1\right)$ gave impure $( \pm)$-II-10 (123 mg, $0.118 \mathrm{mmol}, 76 \%$ ). The impure solid was washed with EtOAc/Hexane (2:1) (1 mL), centrifuged, the supernatant decanted and the residue dried at high vacuum to obtain pure $( \pm)$-II-10 as a white solid (98 mg, $0.094 \mathrm{mmol}, 60 \%)$. M.p. $212-214{ }^{\circ} \mathrm{C} . \quad$ TLC $\left(\mathrm{CHCl}_{3} / \mathrm{MeOH} \mathrm{10:1)} R_{\mathrm{f}}\right.$ 0.46. IR (KBr, $\left.\mathrm{cm}^{-1}\right): 3429 \mathrm{~m}, 2983 \mathrm{w}, 2940 \mathrm{w}, 1744 \mathrm{~s}, 1665 \mathrm{~m}, 1596 \mathrm{~m}, 1535 \mathrm{~m}, 1455 \mathrm{~m}$, 1425m, 1253m, 1017m, 910m. ${ }^{1} \mathrm{H}$ NMR (500 MHz, DMSO-d $): 10.20$ (s, 2H), 7.89 $(\mathrm{d}, J=7.5,4 \mathrm{H}), 7.68(\mathrm{~s}, 2 \mathrm{H}), 7.61(\mathrm{~d}, J=7.9,2 \mathrm{H}), 7.60-7.50(\mathrm{~m}, 2 \mathrm{H}), 7.50-7.40(\mathrm{~m}$, $4 \mathrm{H}), 7.23(\mathrm{~d}, J=8.2,2 \mathrm{H}), 5.80(\mathrm{~d}, J=16.2,2 \mathrm{H}), 4.65-4.40(\mathrm{~m}, 10 \mathrm{H}), 4.23(\mathrm{q}, J=6.9$, $4 \mathrm{H}), 4.20(\mathrm{q}, J=6.9,4 \mathrm{H}), 1.24(\mathrm{t}, J=6.9,6 \mathrm{H}), 1.20(\mathrm{t}, J=6.9,6 \mathrm{H}) .{ }^{13} \mathrm{C} \mathrm{NMR}(100$ MHz, DMSO- $\left.d_{6}\right): \delta 165.4,165.0,164.1,154.1,154.0,138.7,136.5,134.7,131.5$, $131.2,129.9,128.2,127.5,121.3,119.3,80.0,78.5,64.3,63.7,47.3,44.9,44.2,13.6$, 
13.5. MS (FAB, magic bullet): $m / z 1039\left(100,[\mathrm{M}+\mathrm{H}]^{+}\right)$. HR-MS (FAB, magic bullet, PEG, Li): $m / z 1045.3678$ ([M + Li] ${ }^{+}, \mathrm{C}_{52} \mathrm{H}_{50} \mathrm{~N}_{10} \mathrm{O}_{14} \mathrm{Li}$, calcd 1045.3668).

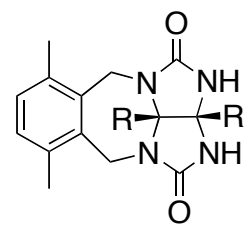

Compound ( \pm )-II-20: A mixture of $p$-xylene $(600 \mathrm{~mL})$ and PTSA (30.84 g, $162.1 \mathrm{mmol})$ was refluxed under an addition funnel filled $( \pm)-11-20 \mathrm{R}=\mathrm{CO}_{2} \mathrm{Et}$ with $4 \AA$ molecular sieves for $1 \mathrm{~h}$. Compound II-19 $(6.00 \mathrm{~g}, 16.21$ mmol) was added in one portion and reflux was continued for $4 \mathrm{~h}$. The reaction mixture was cooled to room temperature and $p$-xylene was distilled off under high vacuum. The residue was dissolved in $\mathrm{CHCl}_{3}(1000 \mathrm{~mL})$, washed with sat. aq. $\mathrm{Na}_{2} \mathrm{CO}_{3}$, dried over anh. $\mathrm{MgSO}_{4}$, and concentrated. Flash chromatography $\left(\mathrm{SiO}_{2}\right.$, $\mathrm{CHCl}_{3} / \mathrm{MeOH}$ 50:1) gave impure ( $( \pm$ )-II-20 as a yellow solid. The impure solid was washed with EtOAc $(10 \mathrm{~mL})$, centrifuged, the supernatant decanted and the residue dried at high vacuum to obtain pure $( \pm)-\mathbf{I I}-20$ as a white solid $(2.45 \mathrm{~g}, 5.88 \mathrm{mmol}$, 36\%). M.p. $250-251^{\circ} \mathrm{C} . \mathrm{TLC}\left(\mathrm{CHCl}_{3} / \mathrm{MeOH} 25: 1\right) R_{\mathrm{f}} 0.25 . \mathrm{IR}\left(\mathrm{KBr}, \mathrm{cm}^{-1}\right): 3432 \mathrm{~m}$, $3221 \mathrm{~m}, 2982 \mathrm{w}, 2927 \mathrm{w}, 1755 \mathrm{~s}, 1707 \mathrm{~s}, 1475 \mathrm{~m}, 1448 \mathrm{~m}, 1278 \mathrm{~m}, 1269 \mathrm{~m}, 1150 \mathrm{~m}$, 1044m. ${ }^{1} \mathrm{H}$ NMR (400 MHz, DMSO- $\left.d_{6}\right): 8.43$ (s, 2H), 6.98 (s, 2H), 4.87 (d, $J=16.0$, 2H), 4.30-4.20 (m, 4H), $4.15(\mathrm{q}, J=7.1,2 \mathrm{H}), 2.44(\mathrm{~s}, 6 \mathrm{H}), 1.26(\mathrm{t}, J=7.1,3 \mathrm{H}), 1.21$ (t, $J=7.1,3 \mathrm{H}) .{ }^{13} \mathrm{C} \mathrm{NMR}\left(100 \mathrm{MHz}, \mathrm{CDCl}_{3}\right): 166.5,166.4,157.8,135.7,135.1$, 130.7, 83.1, 74.3, 64.0, 63.6, 40.4, 20.5, 14.4, 14.2. MS (FAB, PEG): $\mathrm{m} / \mathrm{z} 417$ (100, $\left.[\mathrm{M}+\mathrm{H}]^{+}\right)$. HR-MS (FAB, PEG): $m / z 417.1778\left([\mathrm{M}+\mathrm{H}]^{+}, \mathrm{C}_{20} \mathrm{H}_{25} \mathrm{~N}_{4} \mathrm{O}_{6}\right.$, calcd 417.1774). 


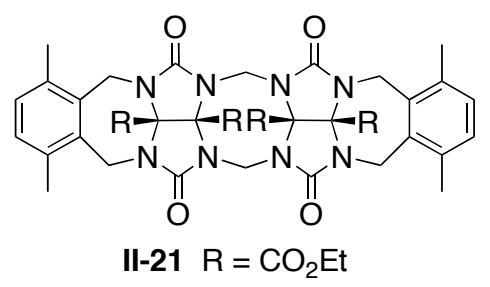

Compound II-21: A mixture of PTSA $(7.30 \mathrm{~g}, 38.4$ mmol) in $\mathrm{ClCH}_{2} \mathrm{CH}_{2} \mathrm{Cl}(400 \mathrm{~mL})$ heated under $\mathrm{N}_{2}$ at reflux for $1 \mathrm{~h}$. under an addition funnel filled with

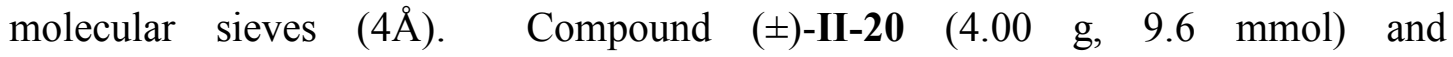
paraformaldehyde $(864 \mathrm{mg}, 28.8 \mathrm{mmol})$ were added in one portion and reflux was continued for $48 \mathrm{~h}$. The reaction mixture was diluted with $\mathrm{CHCl}_{3}(1000 \mathrm{~mL})$, washed with sat. aq. $\mathrm{Na}_{2} \mathrm{CO}_{3}$, dried over anh. $\mathrm{MgSO}_{4}$, and concentrated. The residue was washed with $\mathrm{CH}_{3} \mathrm{CN}(10 \mathrm{~mL})$, centrifuged, the supernatant decanted, and the residue was dried under high vacuum to obtain II-21 $(3.21 \mathrm{~g}, 3.73 \mathrm{mmol}, 78 \%)$ as white solid. M.p. $309-310^{\circ} \mathrm{C}$. TLC $\left(\mathrm{CHCl}_{3} / \mathrm{MeOH}\right.$ 50:1) $R_{\mathrm{f}}$ 0.43. IR $\left(\mathrm{KBr}, \mathrm{cm}^{-1}\right): 2951 \mathrm{w}$, 1738s, 1455s, 1434m, 1249s, 1016m, 908m. ${ }^{1} \mathrm{H}$ NMR (400 MHz, CDCl $): 6.96$ (s, $4 \mathrm{H}), 6.01(\mathrm{~d}, J=16.0,2 \mathrm{H}), 5.17(\mathrm{~d}, J=16.0,4 \mathrm{H}), 4.66(\mathrm{~d}, J=16.0,2 \mathrm{H}), 4.25-4.10$ (m, 12H), 2.47 (s, 12H), 1.35-1.20 (m, 12H). ${ }^{13} \mathrm{C}$ NMR (100 MHz, $\left.\mathrm{CDCl}_{3}\right): 166.5$, $165.5,155.2,135.8,135.2,130.9,80.0,79.4,64.1,63.7,48.2,40.9,20.7,14.4,14.3$. MS (FAB, PEG/CsI): $m / z 989\left(100,[\mathrm{M}+\mathrm{Cs}]^{+}\right)$. HR-MS (FAB, PEG CsI): $m / z$ $989.2440\left([\mathrm{M}+\mathrm{Cs}]^{+}, \mathrm{C}_{42} \mathrm{H}_{48} \mathrm{~N}_{8} \mathrm{O}_{12} \mathrm{Cs}\right.$, calcd 989.2446).

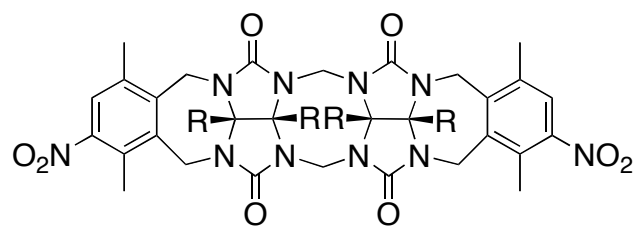

II-22 $\mathrm{R}=\mathrm{CO}_{2} \mathrm{Et}$

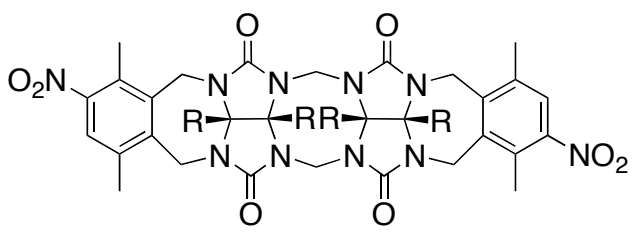

$( \pm)-\mathrm{II}-23 \mathrm{R}=\mathrm{CO}_{2} \mathrm{Et}$

Compound II-22 and ( \pm )-II-23: A mixture of compound II-21 (1.80 g, $2.1 \mathrm{mmol})$ in TFA $(35 \mathrm{~mL})$ was cooled to $0^{\circ} \mathrm{C}$ using ice-water bath. To this mixture $\mathrm{HNO}_{3}(35$ 
$\mathrm{mL}$ ) was added dropwise while stirring. The ice bath was removed after $15 \mathrm{~min}$ and stirring was continued for $4 \mathrm{~h}$. The reaction was quenched with sat. aq. $\mathrm{Na}_{2} \mathrm{CO}_{3}$ and extracted with $\mathrm{CHCl}_{3}(500 \mathrm{~mL})$. The extracts were washed with brine, dried over anh. $\mathrm{MgSO}_{4}$, and concentrated. Flash chromatography $\left(\mathrm{SiO}_{2}, \mathrm{CHCl}_{3}\right)$ gave compound ( $( \pm)-$ II-23 (750 mg, $0.791 \mathrm{mmol}, 38 \%$ ) and compound II-22 (860 mg, $0.908 \mathrm{mmol}, 42 \%)$ as white solids. Compound ( \pm )-II-23: M.p. $288-290{ }^{\circ} \mathrm{C} . \quad \mathrm{TLC}\left(\mathrm{CHCl}_{3} / \mathrm{MeOH} 50: 1\right)$ $R_{\mathrm{f}} 0.46 . \mathrm{IR}\left(\mathrm{KBr}, \mathrm{cm}^{-1}\right): 2981 \mathrm{w}, 1740 \mathrm{~s}, 1529 \mathrm{~m}, 1450 \mathrm{~s}, 1364 \mathrm{~m}, 1254 \mathrm{~s}, 1020 \mathrm{~m}, 911 \mathrm{~m}$. ${ }^{1} \mathrm{H}$ NMR (400 MHz, $\left.\mathrm{CDCl}_{3}\right): 7.45$ (s, 2H), 5.97 (d, $\left.J=16.0,2 \mathrm{H}\right), 5.28$ (d, $J=16.4$, $2 \mathrm{H}), 5.23(\mathrm{~d}, J=16.4,2 \mathrm{H}), 4.68(\mathrm{~d}, J=16.0,2 \mathrm{H}), 4.30-4.15(\mathrm{~m}, 12 \mathrm{H}), 2.56(\mathrm{~s}, 6 \mathrm{H})$, $2.52(\mathrm{~s}, 6 \mathrm{H}), 1.36(\mathrm{t}, J=7.1,6 \mathrm{H}), 1.30(\mathrm{t}, J=7.1,3 \mathrm{H}) .{ }^{13} \mathrm{C} \mathrm{NMR}\left(100 \mathrm{MHz}, \mathrm{CDCl}_{3}\right)$ : $166.1,165.2,155.1,155.0,150.9,140.4,138.9,136.4,129.0,125.7,79.5,79.4,64.3$, 64.1, 48.3, 40.6, 20.6, 15.7, 14.4, 14.3 (only 20 of the 21 expected resonances were observed). MS (FAB, magic bullet, CsI): $m / z 1079\left(100,[\mathrm{M}+\mathrm{Cs}]^{+}\right)$. HR-MS (FAB, magic bullet, CsI): $m / z$ 1079.2174 ([M $+\mathrm{Cs}]^{+}, \mathrm{C}_{42} \mathrm{H}_{46} \mathrm{~N}_{10} \mathrm{O}_{16} \mathrm{Cs}$, calcd 1079.2148). Compound II-22: M.p. $273-275{ }^{\circ} \mathrm{C}$. TLC $\left(\mathrm{CHCl}_{3} / \mathrm{MeOH} 50: 1\right) R_{\mathrm{f}} 0.43$. IR ( $\mathrm{KBr}$, $\left.\mathrm{cm}^{-1}\right): 2984 \mathrm{w}, 1750 \mathrm{~s}, 1526 \mathrm{~m}, 1449 \mathrm{~s}, 1366 \mathrm{~m}, 1253 \mathrm{~s}, 1020 \mathrm{~m}, 910 \mathrm{~m} .{ }^{1} \mathrm{H}$ NMR (400 $\left.\mathrm{MHz}, \mathrm{CDCl}_{3}\right): 7.40(\mathrm{~s}, 2 \mathrm{H}), 5.99(\mathrm{~d}, J=16.0,1 \mathrm{H}), 5.98(\mathrm{~d}, J=16.0,1 \mathrm{H}), 5.27(\mathrm{~d}, J=$ $16.4,2 \mathrm{H}), 5.22(\mathrm{~d}, J=16.4,2 \mathrm{H}), 4.69(\mathrm{~d}, J=16.0,2 \mathrm{H}), 4.30-4.15(\mathrm{~m}, 12 \mathrm{H}), 2.53(\mathrm{~s}$, $12 \mathrm{H}), 1.34(\mathrm{t}, J=7.1,6 \mathrm{H}), 1.29$ (t, $J=7.1,6 \mathrm{H}) .{ }^{13} \mathrm{C} \mathrm{NMR}\left(100 \mathrm{MHz}, \mathrm{CDCl}_{3}\right):$ 166.1, 165.2, 155.1, 155.1, 150.8, 140.4, 138.9, 136.4, 129.0, 125.7, 79.6, 79.4, 64.3, 64.0, $48.3,40.6,40.6,20.6,15.7,14.4,14.3$ (only 21 of the 22 expected resonances were observed). MS (FAB, CsI): $m / z 1079\left(100,[\mathrm{M}+\mathrm{Cs}]^{+}\right)$. HR-MS (FAB, magic bullet, CsI): $m / z$ 1079.2177 ([M + Cs] $]^{+}, \mathrm{C}_{42} \mathrm{H}_{46} \mathrm{~N}_{10} \mathrm{O}_{16} \mathrm{Cs}$, calcd 1079.2148). 


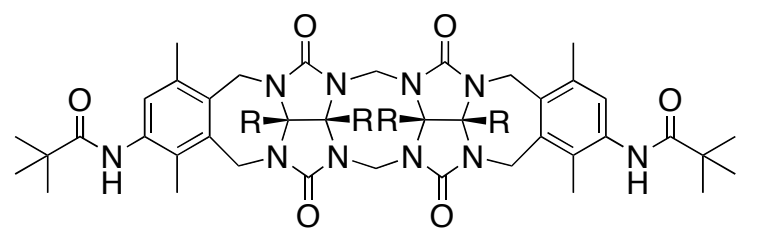

II-9 $\mathrm{R}=\mathrm{CO}_{2} \mathrm{Et}$
Compound II-9: A mixture of compound II-22 (60 mg, $0.063 \mathrm{mmol})$ and $10 \% \mathrm{Pd} / \mathrm{C}(50 \mathrm{mg})$ in anh. DMF (9

$\mathrm{mL})$ was stirred under $\mathrm{H}_{2}(10 \mathrm{psi})$ at $\mathrm{RT}$ for $6 \mathrm{~h}$ in a Schlenk apparatus. The reaction mixture was filtered under Ar and concentrated under high vacuum at RT to obtain a highly air and moisture sensitive diamine as a white solid. The crude solid was dissolved in a mixture of anh. degassed $\mathrm{CH}_{2} \mathrm{Cl}_{2}(8 \mathrm{~mL})$ and distilled $\mathrm{NEt}_{3}(0.5 \mathrm{~mL})$ under Ar. This solution was added to a solution of pivaloyl chloride (37 $\mathrm{mg}, 0.30$ mmol) in anh. degassed $\mathrm{CH}_{2} \mathrm{Cl}_{2}(5 \mathrm{~mL})$ at $-78{ }^{\circ} \mathrm{C}$. After 15 min., the cooling bath was removed and stirring was continued at room temperature for $6 \mathrm{~h}$ under Ar. The reaction mixture was diluted with $\mathrm{CHCl}_{3}(100 \mathrm{~mL})$, washed with sat. aq. $\mathrm{NaHCO}_{3}$, dried over anh. $\mathrm{MgSO}_{4}$, and concentrated. Radial chromatography $\left(\mathrm{SiO}_{2}\right.$, $\mathrm{CHCl}_{3} / \mathrm{MeOH}$ 100:1) gave impure II-9. The impure solid was washed with EtOAc (1 $\mathrm{mL}$ ), centrifuged, the supernatant decanted and the residue dried at high vacuum to obtain pure II-9 as white solid (50 mg, $0.047 \mathrm{mmol}, 75 \%$ ). M.p. $214-217{ }^{\circ} \mathrm{C}$. TLC $\left(\mathrm{CHCl}_{3} / \mathrm{MeOH} 50: 1\right) R_{\mathrm{f}}$ 0.17. IR (KBr, $\left.\mathrm{cm}^{-1}\right): 3429 \mathrm{~m}, 2966 \mathrm{w}, 1748 \mathrm{~s}, 1673 \mathrm{~m}, 1511 \mathrm{~m}$, 1446s, 1367m, 1251s, 1015m, 908m. ${ }^{1} \mathrm{H}$ NMR (400 MHz, DMSO- $\left.d_{6}\right): 8.82(\mathrm{~s}, 2 \mathrm{H})$, $6.85(\mathrm{~s}, 2 \mathrm{H}), 5.73(\mathrm{~d}, J=16.0,2 \mathrm{H}), 4.87(\mathrm{~d}, J=16.0,2 \mathrm{H}), 4.80(\mathrm{~d}, J=16.0,2 \mathrm{H}), 4.56$ $(\mathrm{d}, J=16.0,1 \mathrm{H}), 4.52(\mathrm{~d}, J=16.0,1 \mathrm{H}), 4.36(\mathrm{~d}, J=16.0,2 \mathrm{H}), 4.33(\mathrm{~d}, J=16.0,2 \mathrm{H})$, $4.18(\mathrm{q}, J=7.1,2 \mathrm{H}), 4.12(\mathrm{q}, J=7.1,2 \mathrm{H}), 2.31(\mathrm{~s}, 6 \mathrm{H}), 2.13(\mathrm{~s}, 6 \mathrm{H}), 1.25-1.15(\mathrm{~m}$, 30H). ${ }^{13} \mathrm{C}$ NMR (125 MHz, DMSO- $\left.d_{6}\right): 177.5,166.6,165.3,155.6,155.6,137.6$, $137.1,134.8,133.9,132.0,130.2,80.6,79.9,65.6,65.0,48.6,48.5,39.8,28.6,20.7$, 
$15.3,14.9,14.7$ (only 23 of the 25 expected resonances were observed). MS (FAB, magic bullet, CsI): $m / z 1187\left(100,[\mathrm{M}+\mathrm{Cs}]^{+}\right)$. HR-MS (FAB, magic bullet, CsI): $m / z$ $1187.3807\left([\mathrm{M}+\mathrm{Cs}]^{+}, \mathrm{C}_{52} \mathrm{H}_{66} \mathrm{~N}_{10} \mathrm{O}_{14} \mathrm{Cs}\right.$, calcd 1187.3814).

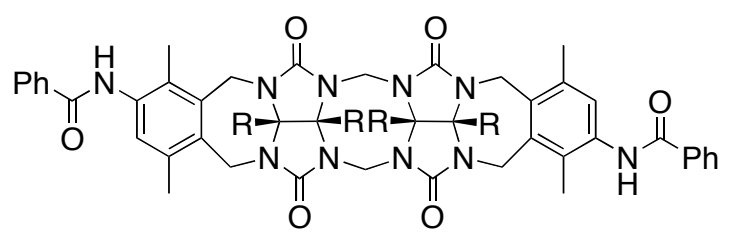

$( \pm)-I I-11 \quad R=\mathrm{CO}_{2} \mathrm{Et}$
Compound ( \pm )-II-11: A mixture of compound $\quad( \pm)-\mathbf{I I}-23 \quad(80 \quad \mathrm{mg}, \quad 0.084$ $\mathrm{mmol})$ and $10 \% \mathrm{Pd} / \mathrm{C}(55 \mathrm{mg})$ in anh.

DMF $(10 \mathrm{~mL})$ was stirred under $\mathrm{H}_{2}(10 \mathrm{psi})$ at $\mathrm{RT}$ for $6 \mathrm{~h}$ in a Schlenk apparatus. The reaction mixture was filtered under $\mathrm{Ar}$ and concentrated under high vacuum at $\mathrm{RT}$ to obtain a highly air and moisture sensitive diamine as a white solid. The crude solid was dissolved in a mixture of anh. degassed $\mathrm{CH}_{2} \mathrm{Cl}_{2}(10 \mathrm{~mL})$ and distilled $\mathrm{NEt}_{3}(0.5$ $\mathrm{mL}$ ) under Ar. This solution was added to a solution of benzoyl chloride $(59 \mathrm{mg}$, $0.41 \mathrm{mmol})$ in anh. degassed $\mathrm{CH}_{2} \mathrm{Cl}_{2}(5 \mathrm{~mL})$ at $-78{ }^{\circ} \mathrm{C}$. After $15 \mathrm{~min}$., the cooling bath was removed and stirring was continued at room temperature for $8 \mathrm{~h}$ under Ar. The reaction mixture was diluted with $\mathrm{CHCl}_{3}(100 \mathrm{~mL})$, washed with sat. aq. $\mathrm{NaHCO}_{3}$, dried over anh. $\mathrm{MgSO}_{4}$, and concentrated. Radial chromatography $\left(\mathrm{SiO}_{2}\right.$, $\mathrm{CHCl}_{3} / \mathrm{MeOH}$ 100:1) gave impure ( \pm )-II-11. The impure solid was washed with EtOAc $(1 \mathrm{~mL})$, centrifuged, the supernatant decanted and the residue dried at high vacuum to obtain pure $( \pm)$-II-11 as a white solid $(67 \mathrm{mg}, 0.061 \mathrm{mmol}, 73 \%)$. M.p. 268-270 ${ }^{\circ} \mathrm{C} . \quad \mathrm{TLC}\left(\mathrm{CHCl}_{3} / \mathrm{MeOH} 50: 1\right) R_{\mathrm{f}}$ 0.26. IR $\left(\mathrm{KBr}, \mathrm{cm}^{-1}\right): 3427 \mathrm{~m}, 2982 \mathrm{w}$, 2930w, 1742s, 1656m, 1520m, 1447s, 1366m, 1255s, 1018m, 907m. ${ }^{1} \mathrm{H}$ NMR (400 MHz, DMSO- $\left.d_{6}\right): 9.89$ (s, 2H), $7.95(\mathrm{~d}, J=7.2,4 \mathrm{H}), 7.58(\mathrm{t}, J=7.2,2 \mathrm{H}), 7.50(\mathrm{t}, J=$ 7.2, 4H), 7.08 (s, 2H), 5.82 (d, $J=16.0,2 \mathrm{H}), 4.97$ (d, $J=16.0,2 \mathrm{H}), 4.89$ (d, $J=16.0$, 
2H), $4.61(\mathrm{~d}, J=16.0,2 \mathrm{H}), 4.45(\mathrm{~d}, J=16.0,2 \mathrm{H}), 4.42(\mathrm{~d}, J=16.0,2 \mathrm{H}), 4.30-4.15$ (m, 8H), $2.40(\mathrm{~s}, 6 \mathrm{H}), 2.28(\mathrm{~s}, 6 \mathrm{H}), 1.27(\mathrm{t}, J=7.1,3 \mathrm{H}), 1.22(\mathrm{t}, J=7.1,3 \mathrm{H}) .{ }^{13} \mathrm{C}$ NMR (125 MHz, DMSO- $\left.d_{6}\right): 165.4,165.2,164.1,154.4,154.3,136.1,135.9,134.3$, $133.7,133.1,131.5,130.7,128.9,128.4,128.3,127.5,79.3,78.7,64.4,63.7,47.3$, 19.6, 14.3, 13.7, 13.5 (only 25 of the 26 expected resonances were observed). MS (FAB, CsI): $m / z 1227\left(100,[\mathrm{M}+\mathrm{Cs}]^{+}\right) . \quad$ HR-MS (FAB, magic bullet, CsI): $m / z$ $1227.3158\left([\mathrm{M}+\mathrm{Cs}]^{+}, \mathrm{C}_{56} \mathrm{H}_{58} \mathrm{~N}_{10} \mathrm{O}_{14} \mathrm{Cs}\right.$, calcd. 1227.3188).

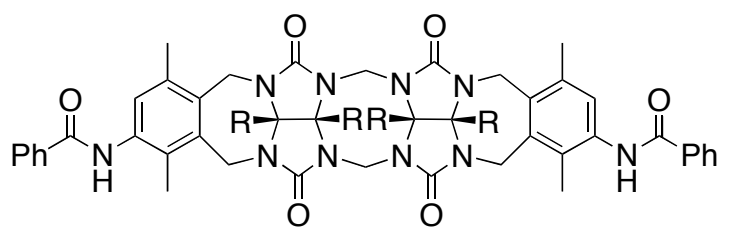

II-8 $\mathrm{R}=\mathrm{CO}_{2} \mathrm{Et}$
Compound II-8: A mixture of compound II-22 (150 mg, $0.158 \mathrm{mmol})$ and $10 \% \mathrm{Pd} / \mathrm{C}(85 \mathrm{mg})$ in anh. DMF (20

$\mathrm{mL})$ was stirred under $\mathrm{H}_{2}(10 \mathrm{psi})$ at $\mathrm{RT}$ for $6 \mathrm{~h}$ in a Schlenk apparatus. The reaction mixture was filtered under Ar and concentrated under high vacuum at RT to obtain a highly air and moisture sensitive diamine as a white solid. The crude solid was dissolved in a mixture of anh. degassed $\mathrm{CH}_{2} \mathrm{Cl}_{2}(15 \mathrm{~mL})$ and distilled $\mathrm{NEt}_{3}(1.3 \mathrm{~mL})$ under Ar. This solution was added to a solution of benzoyl chloride ( $89 \mathrm{mg}, 0.62$ mmol) in anh. degassed $\mathrm{CH}_{2} \mathrm{Cl}_{2}(10 \mathrm{~mL})$ at $-78{ }^{\circ} \mathrm{C}$. After $15 \mathrm{~min}$., the cooling bath was removed and stirring was continued at room temperature for $10 \mathrm{~h}$ under Ar. The reaction mixture was diluted with $\mathrm{CHCl}_{3}(200 \mathrm{~mL})$, washed with sat. aq. $\mathrm{NaHCO}_{3}$, dried over anh. $\mathrm{MgSO}_{4}$, and concentrated. Radial chromatography $\left(\mathrm{SiO}_{2}\right.$, $\mathrm{CHCl}_{3} / \mathrm{MeOH}$ 100:1) gave impure II-8. The impure solid was washed with EtOAc (1 $\mathrm{mL}$ ), centrifuged, the supernatant decanted and the residue dried at high vacuum to obtain pure II-8 as a white solid (112 mg, $0.102 \mathrm{mmol}, 65 \%)$. M.p. $281-284{ }^{\circ} \mathrm{C}$. 
TLC $\left(\mathrm{CHCl}_{3} / \mathrm{MeOH} 50: 1\right) R_{\mathrm{f}} 0.23$. IR $\left(\mathrm{KBr}, \mathrm{cm}^{-1}\right): 3429 \mathrm{~m}, 2928 \mathrm{w}, 1743 \mathrm{~s}, 1650 \mathrm{~m}$, 1519m, 1451s, 1367m, 1254s, 1016m, 909m. ${ }^{1} \mathrm{H}$ NMR (500 MHz, DMSO- $\left.d_{6}\right): 9.88$ (s, 2H), $7.95(\mathrm{~d}, J=7.4,4 \mathrm{H}), 7.59(\mathrm{~m}, 2 \mathrm{H}), 7.50(\mathrm{t}, J=7.6,4 \mathrm{H}), 7.09(\mathrm{~s}, 2 \mathrm{H}), 5.82(\mathrm{~d}$, $J=16.2,2 \mathrm{H}), 4.96(\mathrm{~d}, J=16.2,2 \mathrm{H}), 4.89(\mathrm{~d}, J=16.2,2 \mathrm{H}), 4.62(\mathrm{~d}, J=16.2,1 \mathrm{H})$, $4.59(\mathrm{~d}, J=16.2,1 \mathrm{H}), 4.46(\mathrm{~d}, J=16.2,2 \mathrm{H}), 4.42(\mathrm{~d}, J=16.2,2 \mathrm{H}), 4.24(\mathrm{q}, J=7.1$, $2 \mathrm{H}), 4.18(\mathrm{q}, J=7.1,2 \mathrm{H}), 2.40(\mathrm{~s}, 6 \mathrm{H}), 2.27(\mathrm{~s}, 6 \mathrm{H}), 1.27(\mathrm{t}, J=7.1,3 \mathrm{H}), 1.21(\mathrm{t}, J=$ 7.1, 3H). ${ }^{13} \mathrm{C}$ NMR (125 MHz, DMSO- $\left.d_{6}\right): 165.8,165.8,164.5,154.9,136.5,136.4$, $134.9,134.3,133.5,132.0,131.3,129.4,128.9,128.0,79.8,79.2,64.9,64.2,47.8$, $47.7,20.0,14.8,14.1,14.0$ (only 24 of the 27 expected resonances were observed). MS (FAB, CsI): $m / z 1227\left(100,[\mathrm{M}+\mathrm{Cs}]^{+}\right)$. HR-MS (FAB, magic bullet, CsI): $m / z$ $1227.3192\left([\mathrm{M}+\mathrm{Cs}]^{+}, \mathrm{C}_{56} \mathrm{H}_{58} \mathrm{~N}_{10} \mathrm{O}_{14} \mathrm{Cs}\right.$, calcd 1227.3188).

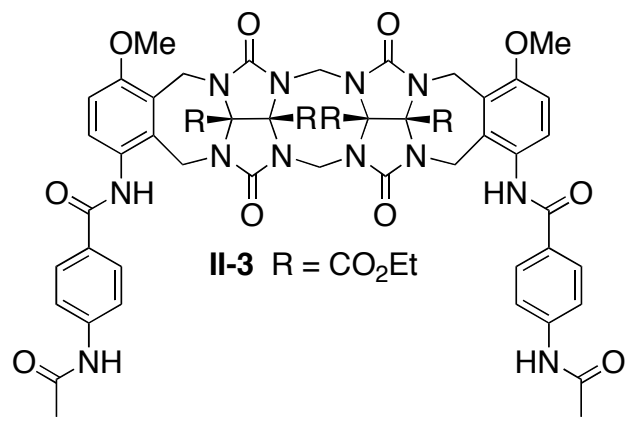

Compound II-3: A mixture of compound II$12(72 \mathrm{mg}, 0.076 \mathrm{mmol})$ and $10 \% \mathrm{Pd} / \mathrm{C}(50$ $\mathrm{mg})$ in anh. DMF (10 mL) was stirred under $\mathrm{H}_{2}$ (10 psi) at RT for $5 \mathrm{~h}$ in a Schlenk apparatus. The reaction mixture was filtered under Ar and concentrated under high vacuum at RT to obtain a highly air and moisture sensitive diamine as a white solid. The crude solid was dissolved in a mixture of anh. degassed $\mathrm{CH}_{2} \mathrm{Cl}_{2}(10 \mathrm{~mL})$ and distilled $\mathrm{NEt}_{3}(4 \mathrm{~mL})$ under Ar. This solution was added to a solution of 4-acetamidobenzoyl chloride $(83 \mathrm{mg}, 0.41 \mathrm{mmol})$ in anh. degassed $\mathrm{CH}_{2} \mathrm{Cl}_{2}(5 \mathrm{~mL})$ at $-78{ }^{\circ} \mathrm{C}$. After $15 \mathrm{~min}$., the cooling bath was removed and stirring was continued at room temperature for $6 \mathrm{~h}$ under Ar. The reaction mixture was 
diluted with $\mathrm{CHCl}_{3}(200 \mathrm{~mL})$, washed with sat. aq. $\mathrm{NaHCO}_{3}$, dried over anh. $\mathrm{MgSO}_{4}$, and concentrated. Radial chromatography $\left(\mathrm{SiO}_{2}, \mathrm{CHCl}_{3} / \mathrm{MeOH}, 100: 4\right.$, then 100:6) gave II-3 as white solid (50 mg, $0.041 \mathrm{mmol}, 53 \%)$. M.p. $>310{ }^{\circ} \mathrm{C}(\mathrm{dec}$.$) . TLC$ $\left(\mathrm{CHCl}_{3} / \mathrm{MeOH} 100: 6\right) R_{\mathrm{f}}$ 0.24. IR $\left(\mathrm{KBr}, \mathrm{cm}^{-1}\right): 3324 \mathrm{~m}, 2959 \mathrm{w}, 2924 \mathrm{w}, 1744 \mathrm{~s}$, 1600m, 1506m, 1456m, 1436m, 1369s, 1258s, 1081m, 1017m. ${ }^{1} \mathrm{H}$ NMR $(400 \mathrm{MHz}$, DMSO- $\left.d_{6}\right): 10.19(\mathrm{~s}, 2 \mathrm{H}), 10.00(\mathrm{~s}, 2 \mathrm{H}), 7.90-7.80(\mathrm{~m}, 4 \mathrm{H}), 7.70-7.60$ (m, 4H), 7.15$7.10(\mathrm{~m}, 2 \mathrm{H}), 7.0-6.95(\mathrm{~m}, 2 \mathrm{H}), 5.75-5.70(\mathrm{~m}, 2 \mathrm{H}), 5.28(\mathrm{~d}, J=16.0,2 \mathrm{H}), 4.75(\mathrm{~d}, J=$ $16.0,2 \mathrm{H}), 4.55(\mathrm{~d}, J=15.8,1 \mathrm{H}), 4.30(\mathrm{~d}, J=15.8,1 \mathrm{H}), 4.25-4.10(\mathrm{~m}, 12 \mathrm{H}), 3.77(\mathrm{~s}$, 6H), $2.06(\mathrm{~s}, 6 \mathrm{H}), 1.25-1.10(\mathrm{~m}, 12 \mathrm{H}) .{ }^{13} \mathrm{C}$ NMR (100 MHz, DMSO- $\left.d_{6}\right): 169.2$, $166.0,165.4,164.4,155.0,154.9,154.4,142.8,134.7,130.7,129.0,128.6,127.9$, $124.9,118.7,118.6,111.9,80.4,79.0,64.9,64.3,56.5,47.9,47.7,36.6,24.6,14.1$, 14.0. MS (FAB, CsI): $m / z 1345\left(100,[\mathrm{M}+\mathrm{Cs}]^{+}\right) . \quad$ HR-MS (FAB, CsI): $m / z$ $1345.3243\left([\mathrm{M}+\mathrm{Cs}]^{+}, \mathrm{C}_{58} \mathrm{H}_{60} \mathrm{~N}_{12} \mathrm{O}_{18} \mathrm{Cs}\right.$, calcd 1345.3203).

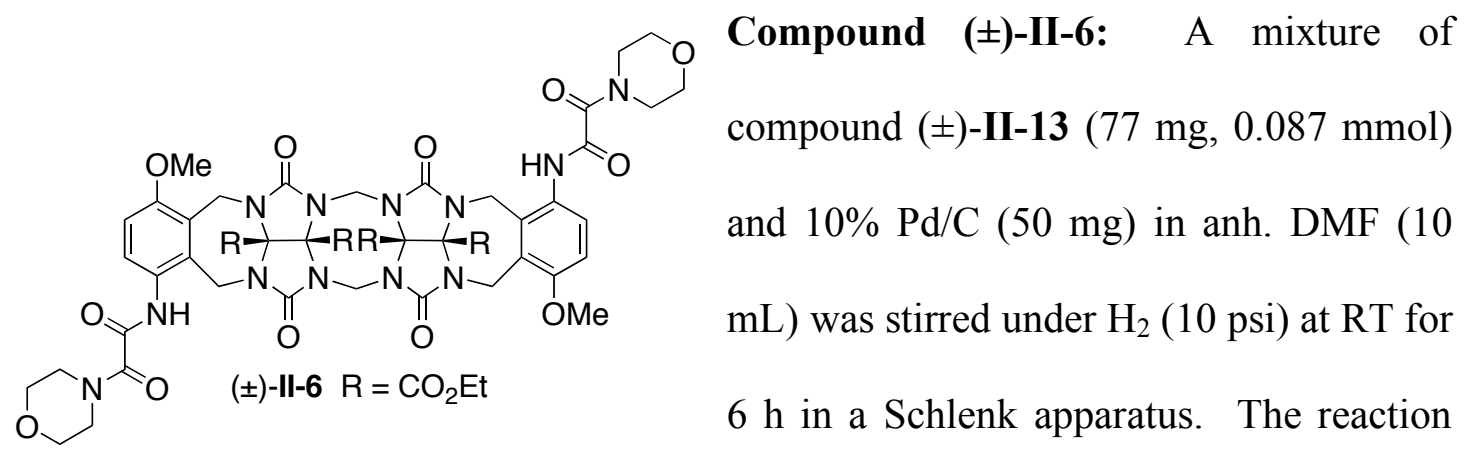

mixture was filtered under Ar and concentrated under high vacuum at RT to obtain a highly air and moisture sensitive diamine as a white solid. The crude solid was 
dissolved in a mixture of anh. degassed $\mathrm{CH}_{2} \mathrm{Cl}_{2}(10 \mathrm{~mL})$ and distilled $\mathrm{NEt}_{3}(0.5 \mathrm{~mL})$ under Ar. This solution was added to a solution of 4-morpholineglyoxyloyl chloride (34 mg, $0.19 \mathrm{mmol})$ in anh. degassed $\mathrm{CH}_{2} \mathrm{Cl}_{2}(5 \mathrm{~mL})$ at $-78{ }^{\circ} \mathrm{C}$. After $15 \mathrm{~min}$., the cooling bath was removed and stirring was continued at room temperature for $6 \mathrm{~h}$ under Ar. The reaction mixture was diluted with $\mathrm{CHCl}_{3}(200 \mathrm{~mL})$, washed with sat. aq. $\mathrm{NaHCO}_{3}$, dried over anh. $\mathrm{MgSO}_{4}$, and concentrated. Radial chromatography $\left(\mathrm{SiO}_{2}, \mathrm{CHCl}_{3} / \mathrm{MeOH} 100: 3\right)$ gave ( $( \pm)$-II-6 as white solid (45 mg, $\left.0.038 \mathrm{mmol}, 43 \%\right)$. M.p. $>330{ }^{\circ} \mathrm{C}\left(\right.$ dec.). $\mathrm{TLC}\left(\mathrm{CHCl}_{3} / \mathrm{MeOH} 100: 4\right) R_{\mathrm{f}} 0.22$. IR $\left(\mathrm{KBr}, \mathrm{cm}^{-1}\right): 3440 \mathrm{~m}$, 2979w, 2925w, 1748s, 1652m, 1455m, 1368w, 1270s, 1115w, 1082w, 1017w. ${ }^{1} \mathrm{H}$ NMR (400 MHz, DMSO- $\left.d_{6}\right): 10.44$ (s, 2H), $7.16(\mathrm{~d}, J=8.9,2 \mathrm{H}), 6.99$ (d, $J=8.9$, 2H), $5.77(\mathrm{~d}, J=16.0,2 \mathrm{H}), 5.17(\mathrm{~d}, J=16.0,2 \mathrm{H}), 4.80(\mathrm{~d}, J=16.0,2 \mathrm{H}), 4.47(\mathrm{~d}, J=$ $16.0,2 \mathrm{H}), 4.32(\mathrm{~d}, J=16.0,2 \mathrm{H}), 4.30(\mathrm{~d}, J=16.0,2 \mathrm{H}), 4.25-4.05(\mathrm{~m}, 8 \mathrm{H}), 3.79(\mathrm{~s}$, $6 \mathrm{H}), 3.70-3.50(\mathrm{~m}, 16 \mathrm{H}), 1.22(\mathrm{t}, J=7.0,3 \mathrm{H}), 1.15(\mathrm{t}, J=7.0,3 \mathrm{H}) .{ }^{13} \mathrm{C} \mathrm{NMR}(100$ MHz, DMSO- $\left.d_{6}\right): 166.0,164.9,163.6,163.4,155.7,155.3,155.0,133.9,128.5$, $127.5,125.6,112.7,80.5,79.5,67.3,66.7,65.3,64.6,57.0,48.2,47.1,42.3,37.0$, 14.5, 14.4. MS (FAB, magic bullet): $m / z 1174\left(100,[\mathrm{M}+\mathrm{H}]^{+}\right)$. HR-MS (FAB, magic bullet, CsI): $m / z 1305.3125\left([\mathrm{M}+\mathrm{Cs}]^{+}, \mathrm{C}_{52} \mathrm{H}_{60} \mathrm{~N}_{12} \mathrm{O}_{20} \mathrm{Cs}\right.$, calcd 1305.3101).

\subsubsection{NMR Experiments.}

NMR spectra were measured on spectrometers operating at 400 or $500 \mathrm{MHz}$ for ${ }^{1} \mathrm{H}$ and 100 or $125 \mathrm{MHz}$ for ${ }^{13} \mathrm{C}$. Temperature was maintained $( \pm 0.5 \mathrm{~K})$ with a 
temperature control module that has been calibrated using the separation of the resonances of methanol. For the self-association measurements, spectra were recorded at a series of concentrations $(10-0.05 \mathrm{mM})$. Spectra for dimeric clips at higher than $10 \mathrm{mM}$ concentration were recorded in $12 \mathrm{~mm}$ microtubes matched with $\mathrm{CDCl}_{3}$. Spectra were referenced relative to residual solvent resonances. 


\section{Chapter 3: Deconvolution of a Multi-Component Interaction Network Using Systems Chemistry.}

\subsection{Introduction.}

Living organisms operate in a multi-component complex environment where various events such as self-regulation, amplification, and hemostasis are regulated through complex networks. ${ }^{108-111}$ Inside the cellular environment, signal transduction proceeds through a sequence of steps that transforms the system from one state to a completely different state. In single cell and multi-cellular organisms the metabolic, signal transduction pathways are guided by various protein-protein interactions that are in turn controlled by genetic regulatory networks. ${ }^{112}$ A recent study revealed that the transcription regulatory networks in yeast Saccharomyces cerevisiae involve 4549 physical interactions between 3278 yeast proteins where as genetic regulatory network is formed by 1289 directed positive or negative direct transcriptional regulations within a set of 68 proteins. ${ }^{113,114}$ Such protein-protein interaction networks define pathways for the propagation of various signals such as phosphorylation and allosteric regulation of proteins. Another study on Escherichia coli was able to identify 1079 regulatory interactions out of which 741 interactions are involved in the network that regulate of amino acid biosynthesis, flagella biosynthesis, osmotic stress response, antibiotic resistance, and iron regulation. ${ }^{115}$ 
Biologists were always inspired to deconvolute these complexities observed in Nature and they adopted a systematic approach - called systems biology - that deals with these emergent properties originated in highly complex interaction networks. ${ }^{116-}$ 123 Systems biologists integrate all fundamental information known about the system from experiments and the literature, formulate mathematical models that describe the structure of such systems and solve those models using computational approaches. ${ }^{124}$ The goal of this approach is not only to understand how the network of dynamic interactions give rise to functional behavior but also to apply the results in drug discovery, pharmacology, and the clinic. ${ }^{125-127}$ Such inherent complexities observed in Nature inspired supramolecular chemists to design systems that mimic at least some of those complexities. In the past decade, due to technical difficulties it was difficult to analyze complex systems with high level of accuracy. With the discovery of highly sensitive modern analytical techniques, now it is possible to overcome the technical obstacles to analyze a multi-component mixture precisely. Due to these technical advancements complex systems are among the growing areas in current research in chemistry. ${ }^{63,128-133} \mathrm{We}$, and others, are involved in the development of systems chemistry that deals with mutually interacting multi-component chemical systems. ${ }^{134-137}$

Our group reported a 12-component mixture that undergoes thermodynamically controlled social self-sorting system in water based on directional metal-ligand interactions and less directional ion-dipole, electrostatic, charge transfer interactions, as well as the hydrophobic effect. ${ }^{16}$ In contrast to the systems mentioned above, Natural and biological systems are driven by kinetically controlled processes. 
Subsequently, we generated a high fidelity kinetic self-sorting system comprising $\mathrm{CB}[6], \mathrm{CB}[7]$, and guests containing two binding epitopes. We analyzed the kinetic behavior and demonstrated how such systems respond to external stimuli by changing their configuration over time from one particular state to another particular state. In the next step, our aim was to mimic another important phenomena that is commonplace in Nautre - biological networks. In the domain of supramolecular chemistry, due to extremely high $\mathrm{K}_{\mathrm{a}}$ values (up to $\left.10^{15} \mathrm{M}^{-1}\right)$ and selectivities $\left(\mathrm{K}_{\text {rel }}\right.$ up to $10^{7}$ ) observed for $\mathrm{CB}[\mathrm{n}]$ host-guest pairs ${ }^{31}$ we realized that the presence of a selfsorted state under thermodynamic control in an $n$-component mixture does not say anything about the composition of the intermediate states comprising $1,2,3, \ldots . \mathrm{n}-1$ components. The identities of the host-guest pairs present within smaller mixtures can and do in many cases differ dramatically from those present in the n-component mixture. Such intermediate states would be highly responsive to the presence (addition, removal, or transformation) of new components, which may lead to drastically different state. As a starting point toward mimicking the complex web of recognition events that governs Natural systems we wondered whether an 8component self-sorting mixture comprising III-1 - III-8 could be built up in eight steps by sequential addition of components in a manner that would result in selfsorted states at every step along the way. In this chapter, we have we used four hosts III-1 - III-4 and four guests III-5 - III-8 and added them in arbitrary sequences to create an artificial complex interaction network that operates under thermodynamic control and implemented a systems level approach that allowed us to understand the complexities of this system. 


\subsection{Preparation of an 8-Component Self-Sorted Mixture.}

\subsubsection{Selection of the Chemical Components Used in this Study.}

To design an 8-component social self-sorting mixture, we selected three members of the cucurbit[n] uril family (III-6 - III-8) because it is well-known that $\mathrm{CB}[\mathrm{n}]$ compounds bind cationic guests with high affinity and selectivity in water. $^{138,139}$ We choose $\beta$-cyclodextrin $(\beta-\mathrm{CD})$ III-5 as the fourth host in our study because it is commercially available and binds to a wide range of guests with low selectivity. ${ }^{140}$ After some experimentation we selected compounds III-1 - III-4 as our guests. Of critical importance in the selection of III-1 - III-4 were: 1) their hostguest complexes should undergo slow exchange on the chemical shift time scale and 2) exhibit distinct changes in chemical shift upon complexation such that the composition of the mixture can be conveniently monitored by ${ }^{1} \mathrm{H}$ NMR spectroscopy.

CHART III-1. Compounds Used in this Study.

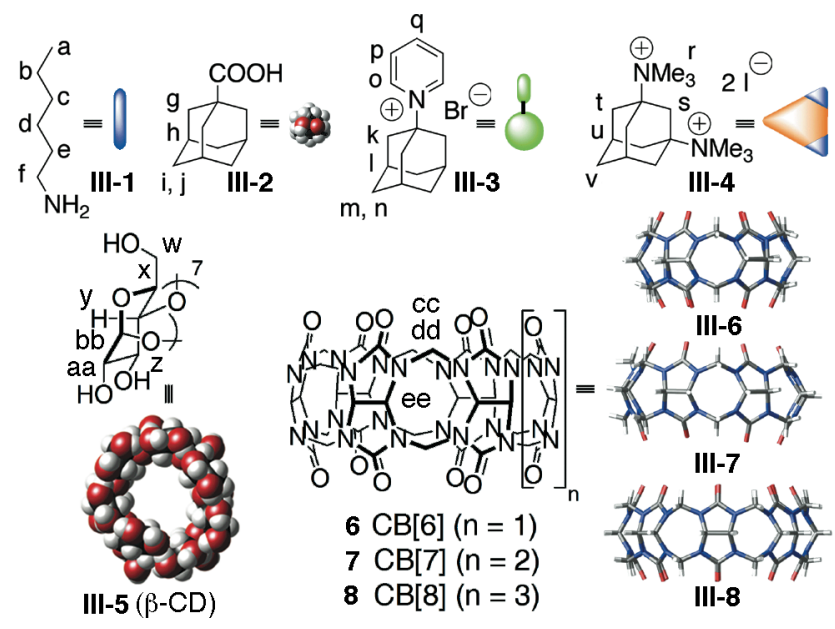




\subsubsection{Self-Sorting System Comprising III-1 - III-8.}

The critical requirement of mimicking the network of cascade interactions in biology is to achieve a well-organized configuration of the system at the end of the transformation. The first step of our study was to ensure a self-sorted state of the final 8-component mixture. The ${ }^{1} \mathrm{H}$ NMR spectra of III-6•III-1, III-5•III-2, III7•III-3, and III-8•III-4, and a mixture of III-1 - III-8 are shown in Figure III-1. The

${ }^{1} \mathrm{H}$ NMR spectrum of the mixture (Figure III-1e) is simply equal to the sum of the ${ }^{1} \mathrm{H}$ NMR of its components. ${ }^{16}$ This spectroscopic earmark confirms that the 8 component system comprising guests III-1 - III-4 and hosts III-5 - III-8 undergoes a high fidelity self-sorting process delivering a mixture of III-6•III-1, III-5•III-2, III-7•III-3, and III-8•III-4. 


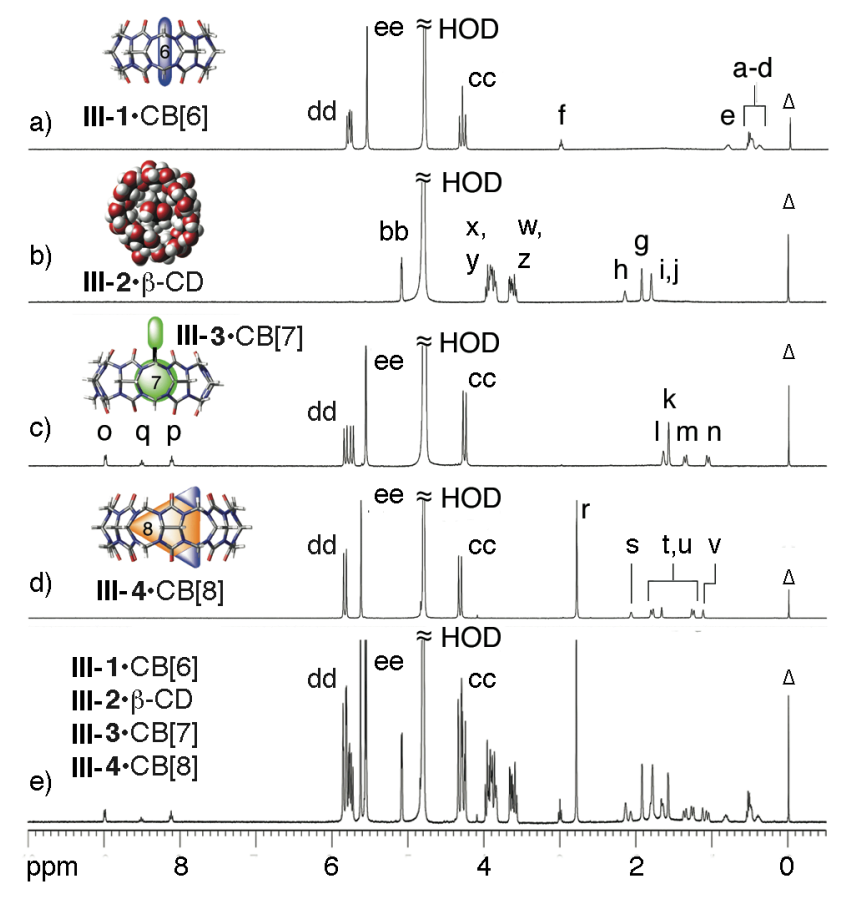

Figure III-1. ${ }^{1} \mathrm{H}$ NMR spectra $\left(400 \mathrm{MHz}, \mathrm{D}_{2} \mathrm{O}, \mathrm{pD} 7.4,298 \mathrm{~K}, 1 \mathrm{mM}\right)$ recorded for: a) III-6•III-1, b) III-5•III-2, c) III-7•III-3, d) III-8•III-4, and e) III-6•III-1, III5•III-2, III-7•III-3, and III-8•III-4. $\left(\mathrm{CD}_{3}\right)_{3} \mathrm{SiCD}_{2} \mathrm{CD}_{2} \mathrm{CO}_{2} \mathrm{D}(\Delta)$ is used as internal standard.

\subsection{Stepwise Construction of 8-Component Self-Sorted Mixture.}

We were gratified that a mixture comprising III-1 - III-8 underwent a high fidelity self-sorting process and decided to explore the stepwise construction of the final 8-component self-sorted state. 


\subsubsection{Molecular Musical Chairs.}

A particularly interesting pathway for the construction of the 8-component self-sorting system comprising III-6•III-1, III-5•III-2, III-7•III-3, and III-8•III-4 involves the stepwise addition of III-1, III-8, III-3, III-5, III-4, III-7, III-2, and then

III-6. The ${ }^{1} \mathrm{H}$ NMR spectra recorded after each of the eight steps are shown in Figure III-2. Remarkably, at each of the seven intermediate steps a single well-defined set of resonances is observed by ${ }^{1} \mathrm{H}$ NMR and each of the intermediate states is therefore self-sorted. This self-sorted pathway is particularly interesting since it proceeds by alternate addition of guest and hosts and resembles the reverse of the game of musical chairs. We whimsically refer to this as a molecular musical chair pathway. ${ }^{141}$ During this molecular musical chair pathway shuttling of guests between hosts is common. For example, in the pathway III-1, III-8, III-3, III-5, III-4, III-7, III-2, III-6 the addition of III-4 to the mixture of III-8 $\cdot$ III-3 and III-5•III-1 triggers the movement of III-3 from III-8 to III-5 with a concommitant dissociation III-1 from III-5 (Figure III-2e). Another example of the shuttling is the behavior of compound III-1 which initially forms a complex with III-8 at the beginning of the pathway whereas in the final mixture it is bound to III-6 and it finds its way to its final destination via III-5 (Figure III-2f). Similarly, guest III-3 initially complexes with host III-8, then takes up residence inside III-5 and finally complexes with III-7 in the 8-component mixture. Compound III-5 plays an important role in this process because it can bind to a wide range of positively charged and neutral guests with modest affinity and low selectivity. As such, III-5 serves as a binding depot for guests during the shuttling between high affinity binding sites in response to the addition of guests. 


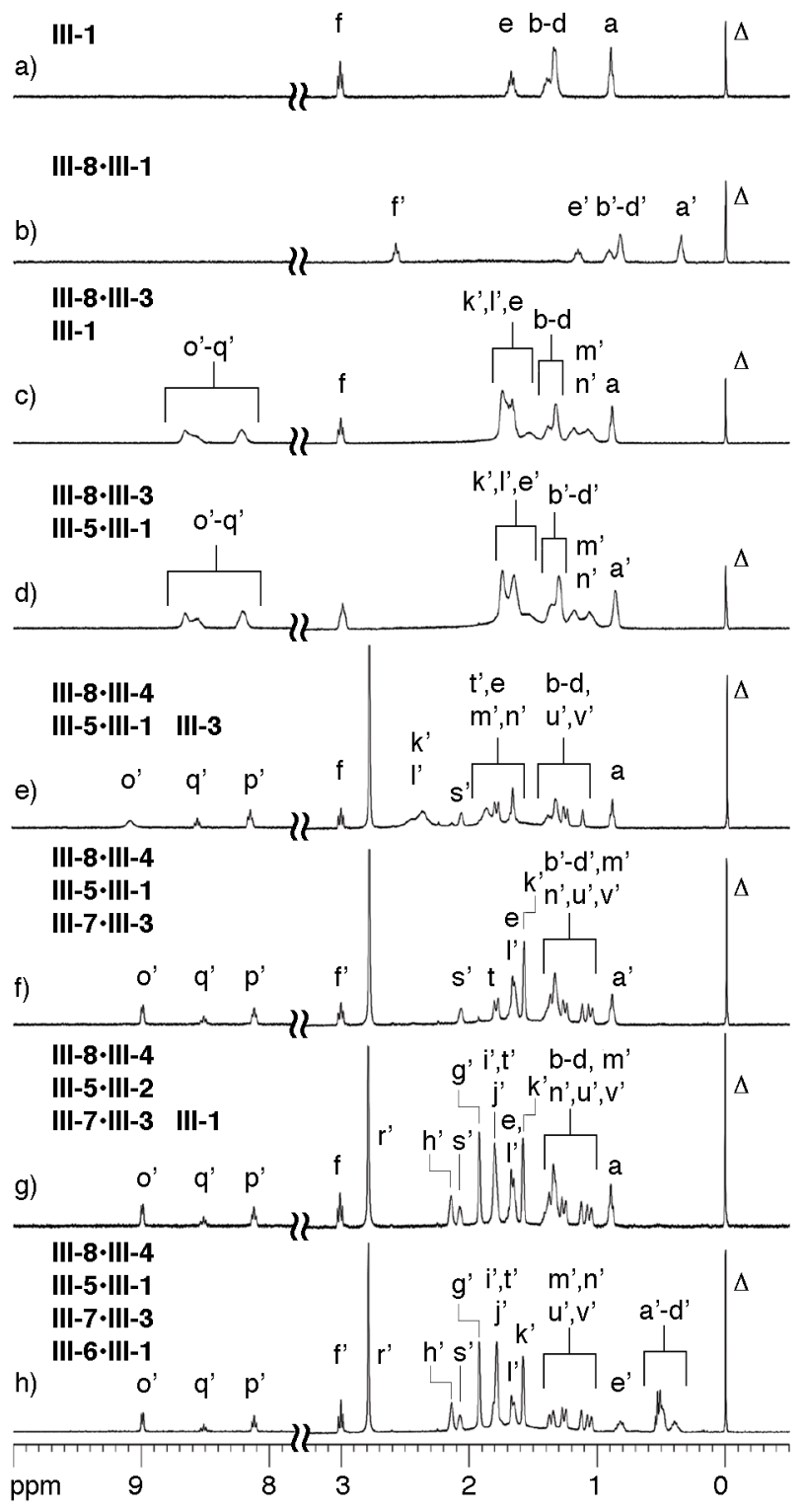

Figure III-2. ${ }^{1} \mathrm{H}$ NMR spectra $\left(400 \mathrm{MHz}, \mathrm{D}_{2} \mathrm{O}, \mathrm{pD} 7.4,298 \mathrm{~K}, 1 \mathrm{mM}\right)$ recorded for an equimolar mixture after addition of 1 eq. of: a) III-1, b) III-8, c) III-3, d) III-5, e) III-4, f) III-7, g) III-2, h) III-6. $\left(\mathrm{CD}_{3}\right)_{3} \mathrm{SiCD}_{2} \mathrm{CD}_{2} \mathrm{CO}_{2} \mathrm{D}(\Delta)$ is used as internal standard. Prime indicates that the guest is complexed with host. 


\subsubsection{Statistical Considerations.}

This section presents some of the statistical considerations involved in the stepwise build-up of a multi-component mixture. Figure III-3 depicts the stepwise construction of two-, three-, and four- component systems. For example, a twocomponent system can be made by the addition of either one of the two components in the first step followed by the addition of the other in the second step (A then B, denoted as $\mathrm{AB}$; $\mathrm{B}$ then $\mathrm{A}$, denoted as $\mathrm{BA}$ ). We refer to these possibilities as pathways, which can be represented by a square (Figure III-3a) where the vertices represent four states and arrows along the edges indicate the direction of increasing number of components. In this stepwise build-up of the two-component mixture, $2^{2}$ states are conceivable (e.g. 0, A, B, and $\mathrm{AB}$ ) and are connected by 2! pathways. Similarly a three-component mixture can be built up in 6 (3!) ways (ABC, $\mathrm{ACB}$, $\mathrm{BAC}, \mathrm{BCA}, \mathrm{CAB}$, and $\mathrm{CBA})$ involving eight $\left(2^{3}\right)$ states. For a three component mixture the various paths and states can be represented by the edges and vertices of a cube (Figure III-3b). By analogy, a four-component mixture can be built up by way of $16\left(2^{4}\right)$ states by adding components by 24 (4!) different pathways. This process can be depicted as a four dimensional hypercube (Figure III-3c). In general, the construction of an $n$-component mixture in $n$ steps involves $2^{n}$ states and there are $n$ ! different pathways to reach the final state. Such an $n$-component system can be represented as an $n$ dimensional hypercube containing $2^{n}$ vertices and $2^{n-1} n$ edges. As the number of components is increased the corresponding hypercube graph reflects the enormous complexity of larger systems (Figure III-4). 

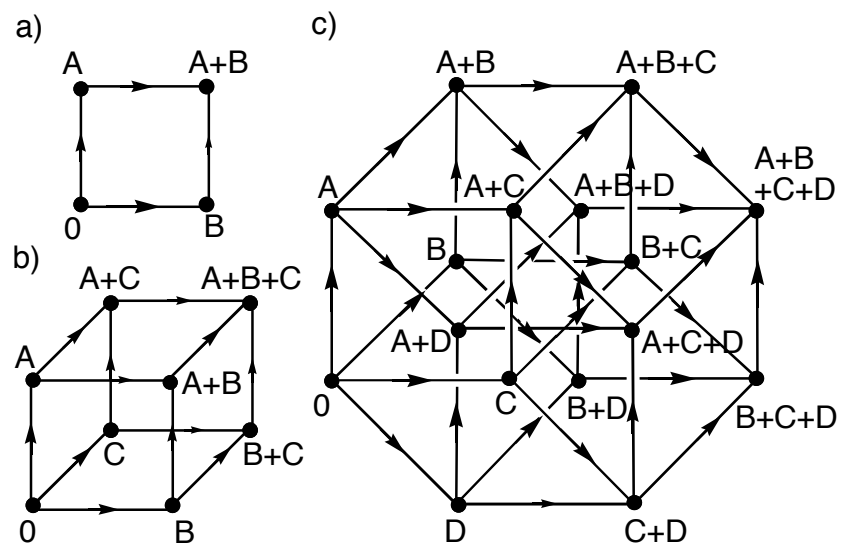

Figure III-3. Schematic representation of the network in stepwise formation of a self-sorted mixture comprising: a) two, b) three, and c) four components.

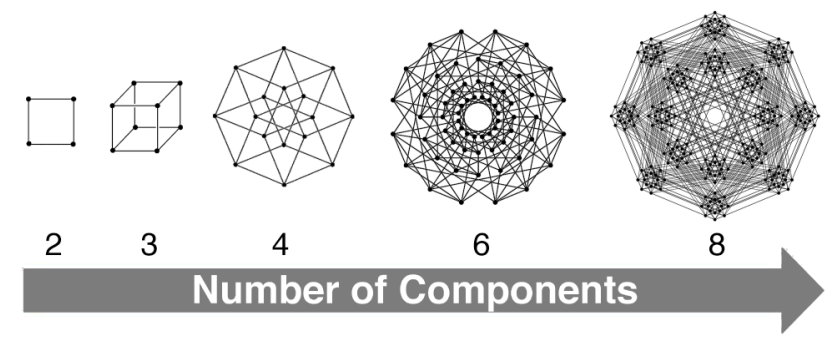

Figure III-4. Schematic representation of 2, 3, 4, 6, and 8-dimensional hypercubes.

\subsubsection{Computational Approach Towards a Global Understanding of the Stepwise Construction of a Four Component System.}

Given that all the experimental systems described in this chapter are at thermodynamic equilibrium, a detailed knowledge of all the initial concentrations and values of $\mathrm{K}_{\mathrm{a}}$ is sufficient for the complete description of the system. These systems are, therefore, quite amenable to computational approaches. In this section we presents the computational characterization of a hypothetical 4-component system 
that sets the stage for deconvolution of the 8-component mixture (III-1 - III-8) described above.

Figure III-5 shows a hypothetical system comprising two hosts (A and B) and two guests $(\mathrm{M}$ and $\mathrm{N})$ subject to the constraints on concentration and values of $\mathrm{K}$ given (Figure III-5b and III-5c). In the simulations, we define a non-self-sorted state as one where one or more components or complexes have mole fractions $0.1 \leq \chi \leq$ 0.9. Conversely, all components and complexes of self-sorted states have mole fractions either less than 0.1 or more than 0.9 . This definition corresponds roughly to our ability to detect minor species by ${ }^{1} \mathrm{H}$ NMR.

A plot of $\Delta G$ versus number of components for each of the $2^{4}$ (16) states of the system is shown in Figure III-5d. We have colored self-sorted states with green dots and non-self-sorted states with red dots. Furthermore, pathways that connect two self-sorted states have been colored green; paths that connect a non-self-sorted state with a self-sorted state or two non-self-sorted states have been colored red. Of the 16 states of this system 14 are self-sorted and even more interesting is the fact that of the 24 pathways for the construction of the four-component mixture, 12 consist entirely of self-sorted states. We refer to them as self-sorted pathways. Several other features of this system deserve comment. First, although the stepwise formation of a multicomponent complex mixture is a function of path, all paths must by definition lead to an identical final state under thermodynamic control. However changing the sequence of addition of components leads to completely different sets of complexes along the way. Second, some pathways may be trivial. For example, addition of 
hosts (e.g. A) followed by their most tight binding guests (e.g. M) does lead to interesting stimuli responsive changes in composition. Third, stimuli responsive movement of guest is common. For example, the state of the system comprising A and $\mathrm{N}$ gives complex $\mathrm{AN}$ after the second step despite the fact that $\mathrm{BN}$ is formed after step 4. The controlled movement of $\mathrm{N}$ from host $\mathrm{A}$ to host $\mathrm{B}-$ driven by the free energy inherent in the 1000 -fold difference in equilibrium constant between $\mathrm{K}_{\mathrm{AM}}\left(10^{9}\right.$ $\left.\mathrm{M}^{-1}\right)$ and $\mathrm{BM}\left(10^{6} \mathrm{M}^{-1}\right)$ - signals the presence of guest $\mathrm{M}$. These observations made in the simulation of this four-component system are conceptually related to the experimental observations made for the 8-component system comprising III-1 - III-8 described above.

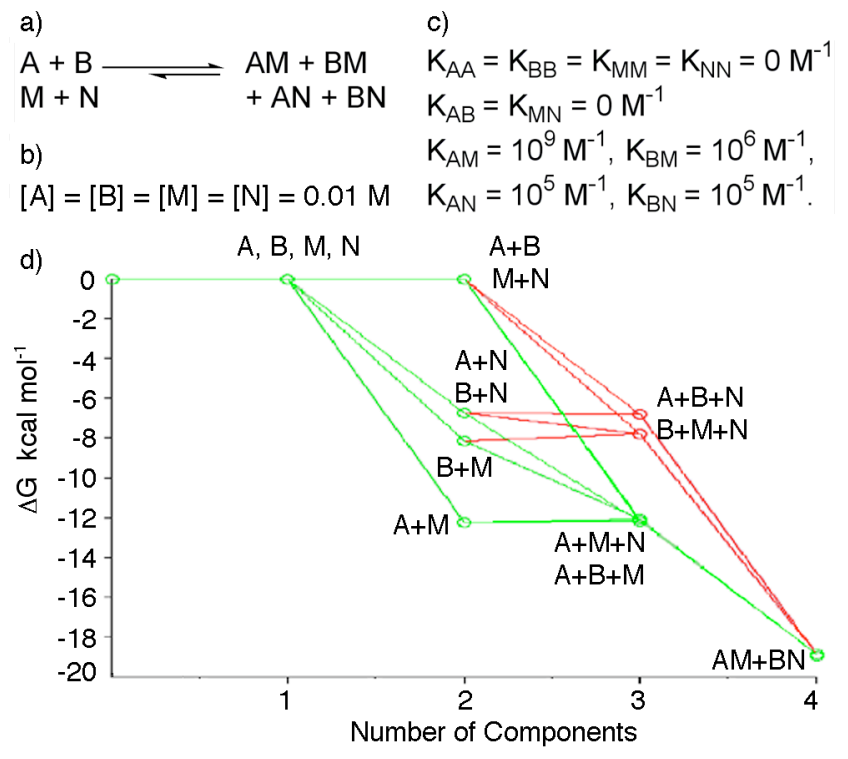

Figure III-5. Stepwise construction of a four component self-sorting mixture: (a) equilibria considered, (b) concentrations of the components, (c) association constants of the various complexes, and (d) a plot of free energy versus number of components. 
3.3.12 Experimental Investigation of Other Pathways that Lead to the Eight Component Self-Sorted System.

Given the successful demonstration of a molecular musical chair pathway and stimulated by the computational results on the four-component system described above, we decided to experimentally investigate some of the pathways that might prove instructional. One such pathway involves addition of the hosts III-5 - III-8 in the first four steps followed by the addition of the guests III-1 - III-4 in the next four steps is depicted in Figure III-6. Although this pathway apparently seems straightforward, there are a few things about it that are noteworthy. Although it is not important to maintain any particular order of addition of hosts (III-5, III-6, III-7, and III-8) in the first four steps since they do not associate with one another, it is critical to maintain a specific sequence (III-4, III-3, III-2, and III-1) in the addition of guests for the remaining states to be self-sorted. If compound III-1 was added prior to the addition of III-3 that would result in a non-self-sorted mixture as III-6 has only 10 fold higher affinity for III-1 than III-7. Although III-3 has 1000 fold higher affinity towards III-7 than III-8, a six-component state containing III-2, III-3, and III-5 III-8 results in a non self-sorted mixture. In the absence of III-4 - which has high selectivity toward III-8 $\left(\mathrm{K}_{\mathrm{a}}=1.11 \times 10^{11} \mathrm{M}^{-1}\right)$ relative to III-7 $\left(\mathrm{K}_{\mathrm{a}}=6.42 \times 10^{4} \mathrm{M}^{-1}\right)$ - compound III-3 will be partially complexed with $\mathrm{CB}[8]\left(\mathrm{K}_{\mathrm{a}}=2.00 \times 10^{9} \mathrm{M}^{-1}\right)$, forcing the formation of III-7-III-2 $\left(\mathrm{K}_{\mathrm{a}}=3.23 \times 10^{8} \mathrm{M}^{-1}\right)$ in the mixture. 


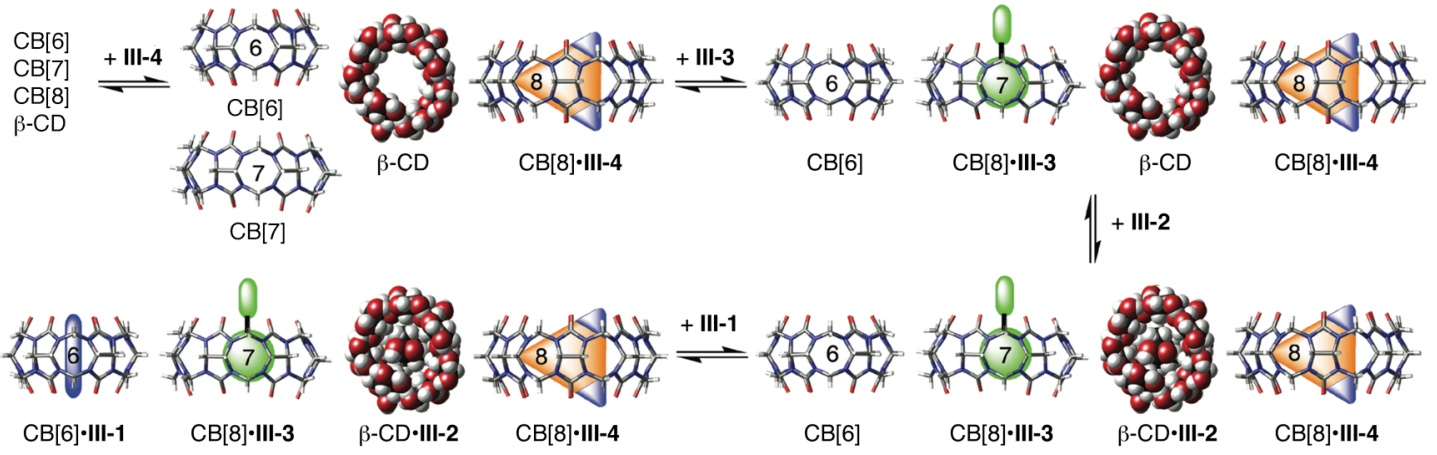

Figure III-6. A pathway that proceeds through eight self-sorted states created by adding all the hosts (III-5 - III-8) in the first four steps followed by the guests (III-4, III-3, III-2, then III-1) in the next four steps.

In contrast to the examples discussed so far, the majority of the pathways are non self-sorted. For example: a pathway involving the alternate addition of host and guest - III-1, III-7, III-2, III-8, III-3, III-5, III-4 then III-6 - proceeds through several non-self-sorted states (Figure III-7). For example, addition of III-2 in the third step leads to a non self-sorted mixture as both guests have substantial affinities towards III-7. Addition of compound III-8 in the fourth step restores the state of the system from non self-sorted to self-sorted by selectively sequestering III-1 to form III-8•III-1 and III-7•III-2. Addition of III-3 in the fifth step lead to a non self-sorted mixture and the system retains its non self-sorted state even after the addition of III-5 in the sixth step. Addition of III-4 resumes self-sorted state of the system in the seventh step. The comparable affinities of guests towards hosts lead to non selfsorted mixture in fifth and sixth step driven by the minimization of the overall free 
energy of the system. The large free energy release upon formation of III-8・III-4 drives III-3 to choose III-7, which in turn drives III-2 to choose III-5.

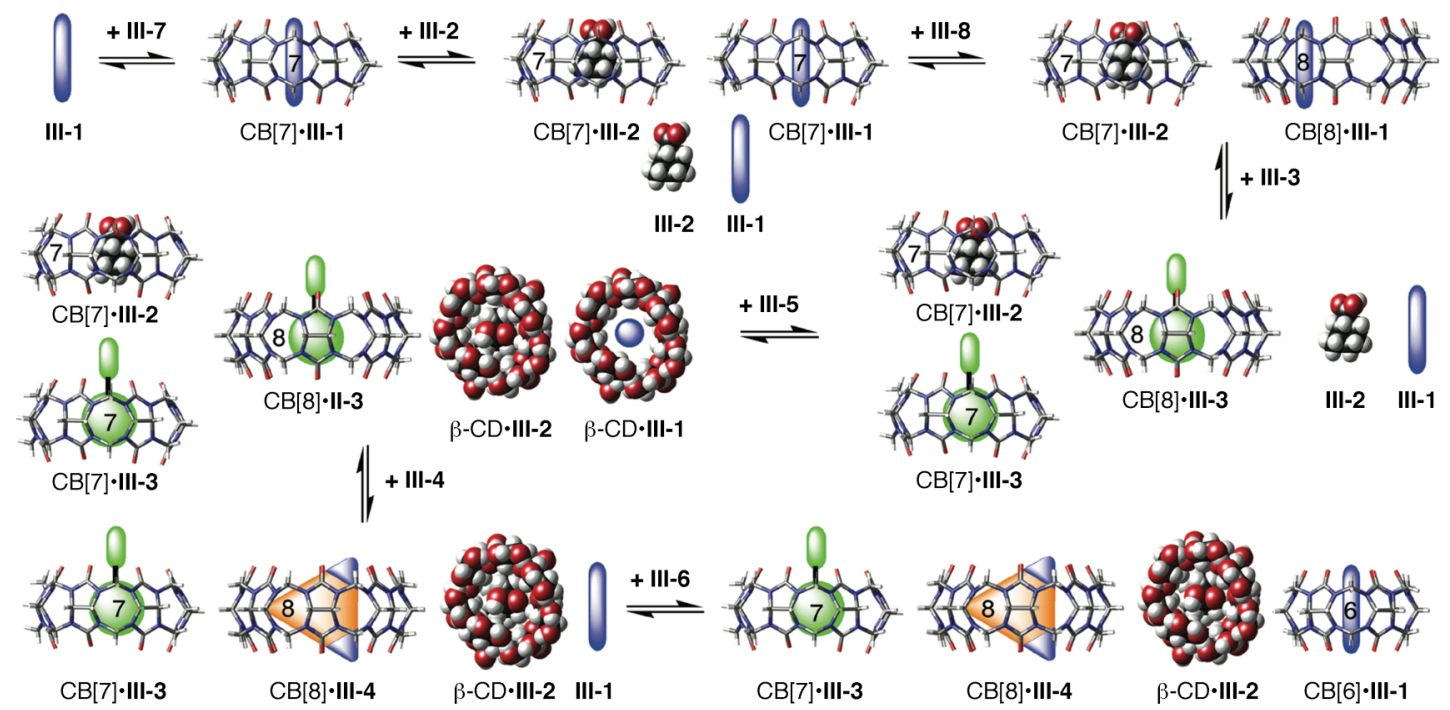

Figure III-7. A pathway created by the alternate addition of hosts and guests that proceeds through some self-sorted states.

\subsubsection{Computational Approach Towards Global Understanding of the Experimental System Comprising III-1 - III-8.}

Examination of the experimental pathways described above provided insights into the formation of multi-component mixtures that were not apparent at the outset of our experiments. Rather than undertaking the unappealing prospect of investigating all 40320 (8!) pathways by experiment - we performed simulations of the system comprising III-1 - III-8 using the values of $\mathrm{K}_{\mathrm{a}}$ shown in Table III-1. The binding constants for III-6•III-1, III-7•III-2, III-7•III-3, III-8・III-3, III-7•III-4, and 
III-8•III-4 were measured experimentally; ${ }^{31}$ we estimated the binding constants of III-1, III-2, III-3, and III-4 for III-5 based on literature precedents; ${ }^{140}$ the binding constants of III-1 with III-7 and III-8 were estimated by extrapolation of results by us and Mock and co-worker. ${ }^{31-33}$

Table III-1: Values of $\mathrm{K}_{\mathrm{a}}\left(\mathrm{M}^{-1}\right)$ of Different Host-Guest Complexes Used In the Simulation.

\begin{tabular}{|l|l|l|l|l|}
\hline & $\beta-C D$ & $\mathrm{CB}[6]$ & $\mathrm{CB}[7]$ & $\mathrm{CB}[8]$ \\
\hline III-1 & 500 & $1 \times 10^{7}$ & $1 \times 10^{6}$ & $1 \times 10^{5}$ \\
\hline III-2 & $1 \times 10^{5}$ & 100 & $3.23 \times 10^{8}$ & 100 \\
\hline III-3 & $1 \times 10^{5}$ & 100 & $1.98 \times 10^{12}$ & $2.0 \times 10^{9}$ \\
\hline III-4 & 100 & 100 & $6.42 \times 10^{4}$ & $1.11 \times 10^{11}$ \\
\hline
\end{tabular}

Figure III-8a shows the free energy of all 256 possible states that may arise in the stepwise formation of an eight-component mixture. As we observed experimentally by ${ }^{1} \mathrm{H}$ NMR, the final state of the simulation represents a self-sorted state. The simulation also provided mole fraction values for each component in the remaining 255 states, which were used to identify whether a particular state was selfsorted or non-self-sorted. In Figure III-8a, self-sorted states are colored green and non self-sorted states are colored red. Although the initial and final states are the same, the intermediate states have distinct sets of complexes populated. This comprehensive knowledge of the compositions of all 256 states allowed us to predict the outcome of all 40320 pathways. Figure III-8b represents a complete interaction 
network - all 40320 pathways - that was previously described by an eight dimensional hypercube.
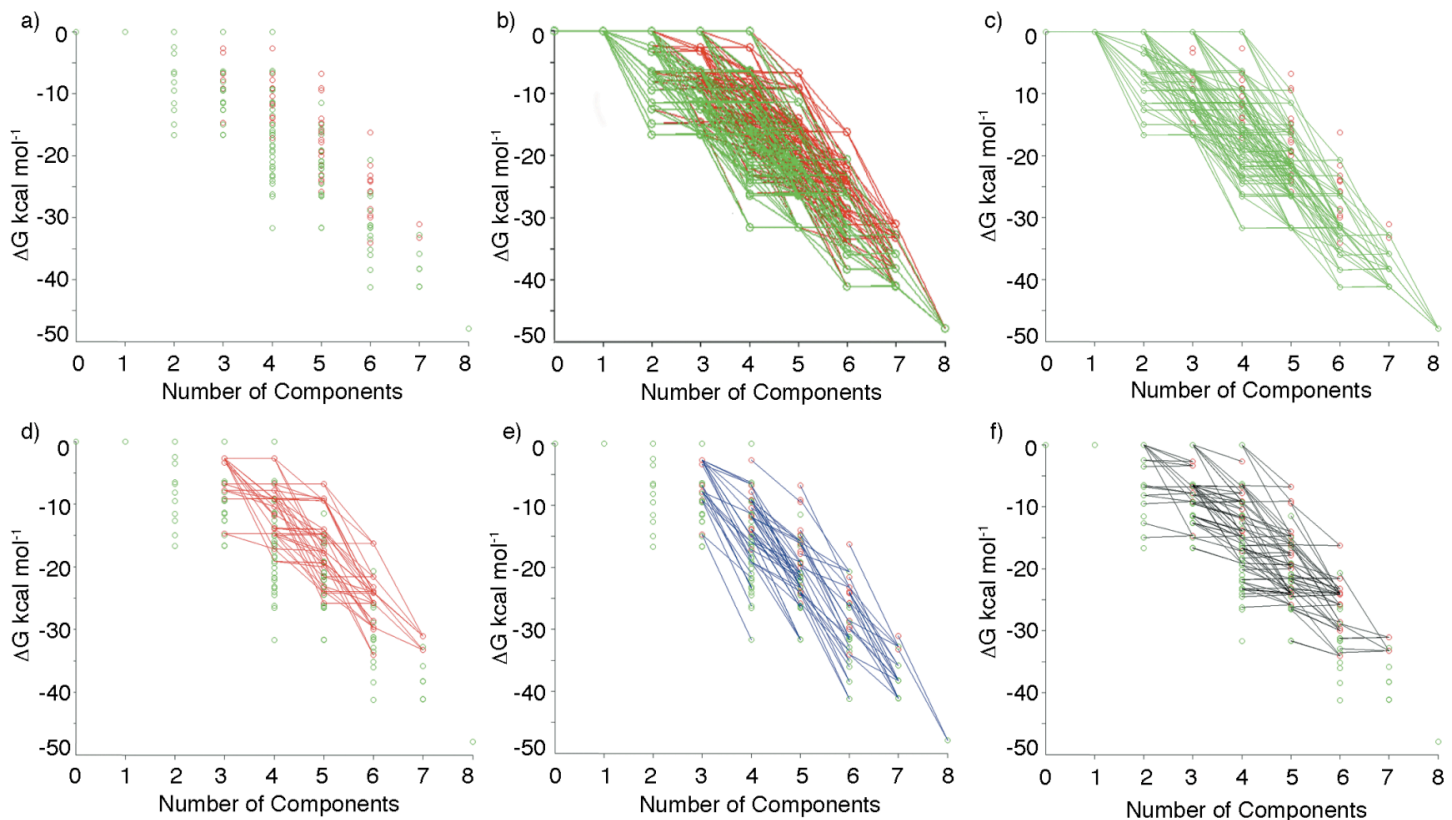

Figure III-8. Stepwise construction of an 8-component self-sorting mixture (concentration of each component $=1 \mathrm{M}$ ): a) all 256 possible states (color coding: self-sorted states, green; non-self-sorted states, red.), b) plot of all 40320 pathways, c) steps that transform a self-sorted state to another self-sorted state, d) steps that transform a non self-sorted state to another non self-sorted state, e) steps that transform a non self-sorted state to another self-sorted state, and f) steps that transform a self-sorted state to another non self-sorted state.

Inspired by the complete deconvolution of the interaction network involved in the stepwise formation of an eight-component self-sorted system, we were interested to have further insight to the subset of these pathways. There are four different kinds of transformations possible upon addition of a single component: one self-sorted state 
to another self-sorted state, one non self-sorted state to another non self-sorted state, one non self-sorted state to another self-sorted state, and one self-sorted state to another non self-sorted state (Figure III-8 c - f). Although the transformation from a self-sorted state to another self-sorted state is observed throughout the pathway (Figure III-8c), transformation from a non self-sorted state to another non self-sorted state is spatially segregated (Figure III-8d) to the upper right hand corner. The formation of a highly organized self-sorted state from a disordered non self-sorted state upon addition of a compound is associated with substantial amount of downhill in free energy (Figure III-8e). In contrast, some transformations from a self-sorted state to another non self-sorted state are associated with a decrease in free energy while others are slightly uphill in energy due to statistical (e.g. entropic) consideration (Figure III-8f). ${ }^{142}$

\subsubsection{Effect of Number of Components on Self-Sorted States.}

We observed several hidden patterns as well as interesting behaviors in the simulation. First, more non self-sorted states emerge in the intermediate steps due to the increase of potentially competing interactions with the increase in the number of components (Table III-2). Only those states that consist of high selectivity and affinity host-guest pairs are self-sorted because they are able to provide sufficient free energy requirements for self-sorting under thermodynamic control (Figure III-8a). 
Table III-2: Total Number of Self-Sorted and Non Self-Sorted States at Different Concentration.

\begin{tabular}{|l|l|l|l|l|l|l|l|}
\hline \multirow{2}{*}{$\begin{array}{l}\text { Number } \\
\text { of } \\
\begin{array}{l}\text { Compo- } \\
\text { nents }\end{array}\end{array}$} & $\begin{array}{l}\text { Number } \\
\text { of States }\end{array}$ & \multicolumn{2}{l|}{ Conc. $(1 \mathrm{M})$} & \multicolumn{2}{l|}{ Conc. $(1 \mathrm{mM})$} & \multicolumn{2}{l|}{ Conc. $(1 \mu \mathrm{M})$} \\
\cline { 2 - 7 } & $\begin{array}{l}\text { Self- } \\
\text { sorted } \\
\text { states }\end{array}$ & $\begin{array}{l}\text { Non } \\
\text { self- } \\
\text { sorted } \\
\text { states }\end{array}$ & $\begin{array}{l}\text { Self- } \\
\text { sorted } \\
\text { states }\end{array}$ & $\begin{array}{l}\text { Non } \\
\text { self- } \\
\text { sorted } \\
\text { states }\end{array}$ & $\begin{array}{l}\text { Self- } \\
\text { sorted } \\
\text { states }\end{array}$ & $\begin{array}{l}\text { Non } \\
\text { self- } \\
\text { sorted } \\
\text { states }\end{array}$ \\
\hline 0 & 1 & 1 & 0 & 1 & 0 & 1 & 0 \\
1 & 8 & 8 & 0 & 8 & 0 & 8 & 0 \\
2 & 28 & 28 & 0 & 25 & 3 & 25 & 3 \\
3 & 56 & 45 & 11 & 39 & 17 & 41 & 15 \\
5 & 70 & 49 & 21 & 44 & 26 & 35 & 35 \\
6 & 28 & 36 & 20 & 32 & 24 & 16 & 40 \\
7 & 8 & 6 & 15 & 14 & 14 & 6 & 22 \\
8 & 1 & 1 & 0 & 1 & 0 & 0 & 8 \\
\hline Total & 256 & 189 & 67 & 170 & 86 & 132 & 124 \\
\hline
\end{tabular}

\subsubsection{Effect of Concentration of Components on Self-Sorted States and Pathways.}

We were interested to quantify how concentration affects the stepwise formation of the self-sorted system since Nature uses concentration as the primary method to control self-assembly processes. As the component concentration is increased from $0.01 \mu \mathrm{M}$ from $1 \mathrm{M}$, we observed an initial decrease in the number of 
self-sorted states followed by an increase above $1 \mu \mathrm{M}$ (Figure III-9). This is due to the fact that at $0.01 \mu \mathrm{M}$ - much below their value of $\mathrm{K}_{\mathrm{d}}$ - many complexes are fully dissociated, while they are only partially complexed around their $K_{d}(1 \mu \mathrm{M}$ concentration), leading to a decrease to the number of non self-sorted states. At higher concentration of the components those host-guest pairs are forced to complex, which result in an increase in the number of self-sorted states. We also observed a substantial effect of concentration on the number of self-sorted pathways. As such completely self-sorted pathways are relatively uncommon. Only 5858 and 3376 pathways are completely self-sorted in $1 \mathrm{M}$ and $1 \mathrm{mM}$ concentration, respectively. The total number of self-sorted pathways can be calculated from Table III-3. Total number of self-sorted pathways $=$ Total number of pathways $\times$ probability of achieving a self-sorted state at each step. When the concentration of the components is $1 \mathrm{M}$, there are total 5858 pathways that are self-sorted. $(40320 \times 45 / 56 \times 49 / 70 \times$ $36 / 56 \times 15 / 28 \times 6 / 8=5858)$. Total number of non self-sorted pathways $=40320-$ $5858=34462]$ Somewhat surprisingly there is no self-sorted pathway at $1 \mu \mathrm{M}$ concentration due to fact that the final state itself becomes non self-sorted at that concentration (Table III-3). These results indicate that by changing the concentration - just as Nature does - we can achieve control over an interaction network in a complex multi-component system. The above study indicates that a significant proportion of biological processes are probably governed by non self-sorted states and pathways due to the involvement of a large number of components in the interaction network inside the cell. 
Table III-3: Effect of Concentration of Components and Sequence of Addition on Pathways.

\begin{tabular}{|c|c|c|c|c|c|c|c|c|c|c|}
\hline \multirow{2}{*}{$\begin{array}{l}\text { Total } \\
\text { Number } \\
\text { of } \\
\text { pathways }\end{array}$} & \multicolumn{3}{|c|}{ Conc. (1 M) } & \multicolumn{4}{|c|}{ Conc. $(1 \mathrm{mM})$} & \multicolumn{3}{|c|}{ Conc. $(1 \mu \mathrm{M})$} \\
\hline & \multicolumn{2}{|c|}{$\begin{array}{l}\text { Self- } \\
\text { sorted } \\
\text { pathways }\end{array}$} & $\begin{array}{l}\text { Non self- } \\
\text { sorted } \\
\text { pathways }\end{array}$ & \multicolumn{2}{|c|}{\begin{tabular}{|l} 
Self- \\
sorted \\
pathways
\end{tabular}} & \multicolumn{2}{|c|}{$\begin{array}{l}\text { Non self- } \\
\text { sorted } \\
\text { pathways }\end{array}$} & $\begin{array}{l}\text { Self- } \\
\text { sorted } \\
\text { pathways }\end{array}$ & \multicolumn{2}{|c|}{$\begin{array}{l}\text { Non self- } \\
\text { sorted } \\
\text { pathways }\end{array}$} \\
\hline \multirow[t]{2}{*}{40320} & \multicolumn{2}{|c|}{5858} & 34462 & \multicolumn{2}{|l|}{3376} & \multicolumn{2}{|c|}{36944} & 0 & \multicolumn{2}{|c|}{40320} \\
\hline & \multicolumn{2}{|c|}{$14.53 \%$} & $85.47 \%$ & \multicolumn{2}{|c|}{$8.38 \%$} & \multicolumn{2}{|c|}{$91.62 \%$} & $0 \%$ & \multicolumn{2}{|c|}{$100 \%$} \\
\hline \multicolumn{2}{|c|}{$\begin{array}{l}\text { Sequence } \\
\text { HHHHGGGG }^{\text {a }}\end{array}$} & \multicolumn{2}{|c|}{$\begin{array}{l}\text { Sequence } \\
\text { GGGGHHHH }^{\text {a }}\end{array}$} & \multicolumn{3}{|c|}{$\begin{array}{l}\text { Sequence } \\
\text { GHGHGHGH }^{\mathrm{a}}\end{array}$} & \multicolumn{2}{|c|}{\begin{tabular}{|l|} 
Sequence \\
HGHGHGHG $^{\mathrm{a}}$
\end{tabular}} & \multicolumn{2}{|c|}{$\begin{array}{l}\text { Random } \\
\text { sequence }^{\mathrm{a}}\end{array}$} \\
\hline $\begin{array}{l}\text { Self- } \\
\text { sorted }\end{array}$ & $\begin{array}{l}\text { Non } \\
\text { self- } \\
\text { sorted }\end{array}$ & $\begin{array}{l}\text { Self- } \\
\text { sorted }\end{array}$ & $\begin{array}{l}\text { Non } \\
\text { self- } \\
\text { sorted }\end{array}$ & $\begin{array}{l}\text { Self- } \\
\text { sorted }\end{array}$ & $\begin{array}{l}\text { Non } \\
\text { self- } \\
\text { sort }\end{array}$ & & $\begin{array}{l}\text { Self- } \\
\text { sorted }\end{array}$ & \begin{tabular}{|l|} 
Non \\
self- \\
sorted
\end{tabular} & $\begin{array}{l}\text { Self- } \\
\text { sorted }\end{array}$ & $\begin{array}{l}\text { Non } \\
\text { self- } \\
\text { sort } \\
\text { ed }\end{array}$ \\
\hline 162 & 414 & 108 & 468 & 68 & 508 & & 86 & 490 & 5434 & $\begin{array}{l}325 \\
82\end{array}$ \\
\hline $28.1 \%$ & $71.9 \%$ & $18.8 \%$ & $81.2 \%$ & $11.8 \%$ & 88.2 & & $14.9 \%$ & $85.1 \%$ & $14.2 \%$ & $\begin{array}{l}85.8 \\
\%\end{array}$ \\
\hline
\end{tabular}

${ }^{\text {a }}$ Concentration of each component $=1 \mathrm{M}$ 


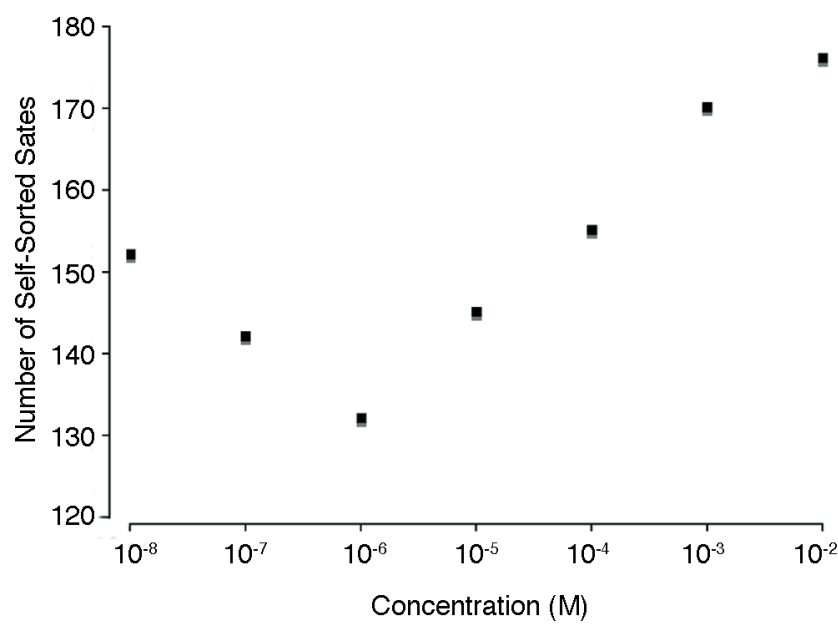

Figure III-9. A plot showing the change in number of self-sorted states with respect to concentration.

\subsubsection{Effect of Sequence of Addition of Components on Pathways.}

Realizing the origin of different behaviors in different pathways we decided to investigate the effect of the order of addition of components on states and pathways. We anticipated that the outcome of the pathways would be very different depending on the order of addition of components (addition of all hosts followed by all guests versus alternate addition of hosts and guests). We investigated the following order of additions by simulation: 1) 576 pathways $(4 \times 3 \times 2 \times 1 \times 4 \times 3 \times 2 \times 1)$ for each of the following addition sequence involving addition of all four hosts followed by the addition of all four guests (HHHHGGGG) and vice versa (GGGGHHHH). 2) 576 pathways $(4 \times 4 \times 3 \times 3 \times 2 \times 2 \times 1 \times 1)$ for each of the two different alternate addition sequence of hosts and guests (GHGHGHGH and HGHGHGHG). Interestingly, we observed that pathways originated from two different kinds of 
addition sequences set themselves apart from each other in the free energy landscape (Figure III-10 a - d). In the sequence HHHHGGGG and GGGGHHHH, there is no change in the free energy observed in the first four steps followed by sharp decrease in free energy observed in every step in next four steps. Whereas, alternate addition sequences (HGHGHGHG or GHGHGHGH) involve a cascade of molecular recognition events that trigger a change of the system from one state to another state.
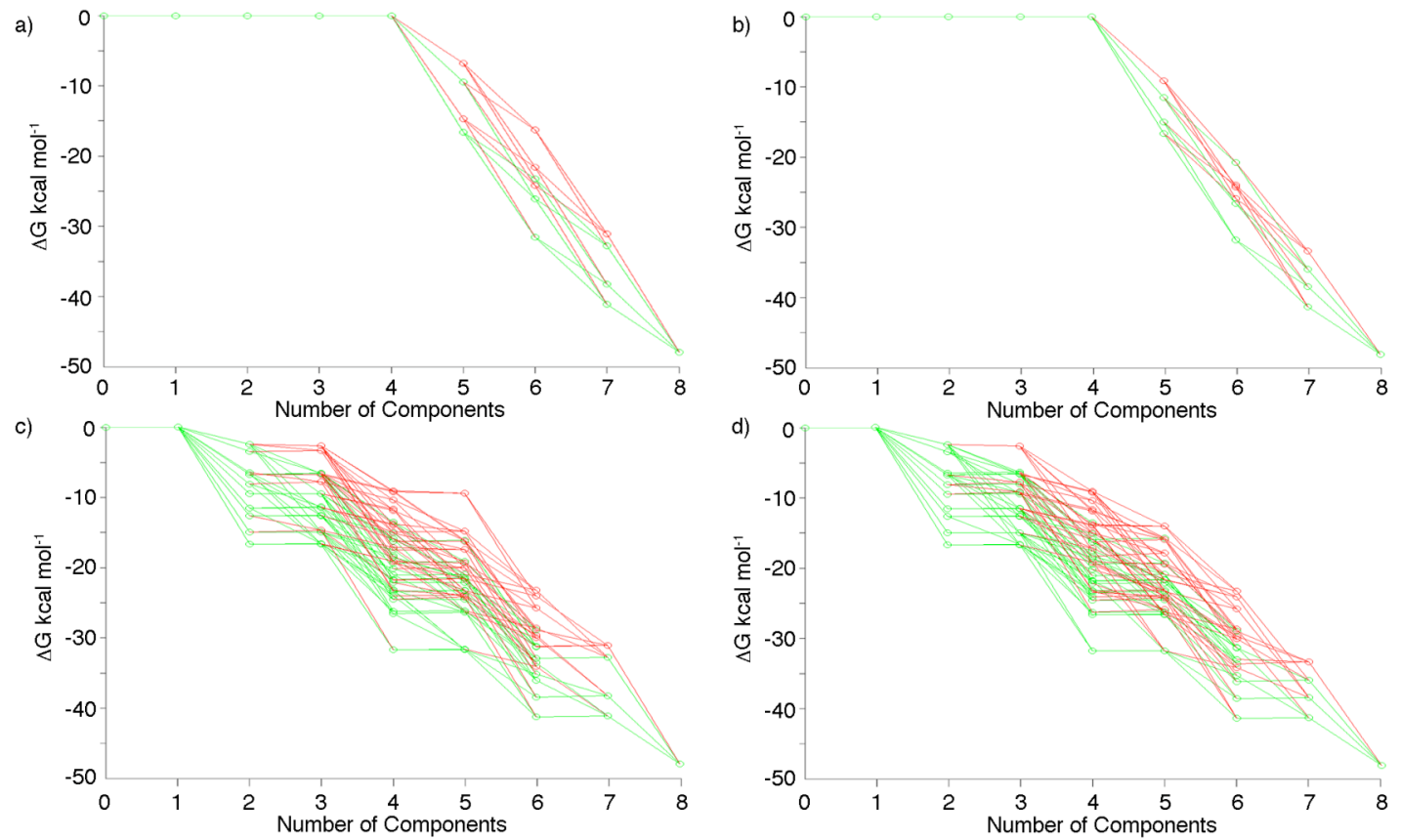

Figure III-10. All 576 pathways for each of the following order of addition (concentration of each component =1 M): (a) GGGGHHHH, (b) HHHHGGGG, (c) GHGHGHGH, and (d) HGHGHGHG. 


\subsection{The Effect of Equilibrium Constants.}

In the previous section we demonstrated that variables like concentration and sequence of addition of components alter the outcome of states and pathways in the stepwise construction of 8-component mixture. We were interested to explore another key variable - equilibrium constant - that Nature uses to control its assembly processes. We derived mean and standard deviations values interactions over a wide range of guests for $\beta-\mathrm{CD}, \mathrm{CB}[6], \mathrm{CB}[7]$, and $\mathrm{CB}[8]$ based on literature precedents (Table III-4). Instead of quantifying individual host-guest interactions by specific numbers (e.g. our experimental system) we incorporated mean \pm standard deviation values and allowed GEPASI to generate a random set of 16 (4 x 4) values and performed simulations similar to our experimental system. When we plotted $\Delta \mathrm{G}$ with respect to number of components we observed similar trend where free energy of the system decreases with the concomitant increase in the number of component (Figure III-11). When we compared 67 simulations from randomly generated binding constants, we observed only $20 \%$ of the final states and approximately $3 \%$ of the total pathways under above conditions are self-sorted (Figure III-12). The mean and standard deviations of host-guest interactions values that we assumed for our hosts are comparable to various interactions observed in biological systems and hence we anticipate our research reflects some of the behaviors observed in biology. 


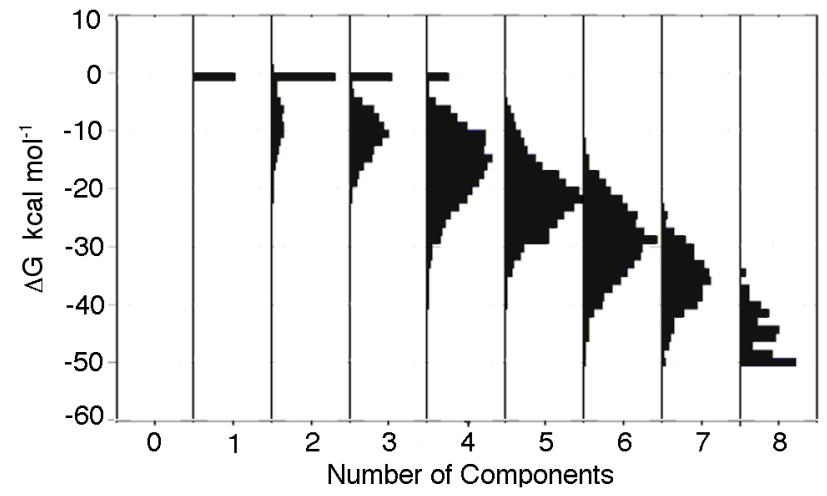

Figure III-11. A histogram showing the distribution of 17152 states derived from randomly generated binding constants for 67 systems.

Table III-4: Range of binding constants of synthetic hosts used in the simulation and summary of binding constants for various interactions observed in biology. ${ }^{143,144}$

\begin{tabular}{|c|c|c|}
\hline Host type & Guest type & Mean $\left(\log \mathrm{K}_{\mathrm{a}}\right)$ \\
\hline$\beta$-cyclodextrin & Organic molecule & $4 \pm 2$ \\
\hline $\mathrm{CB}[6]$ & Organic molecule & $6 \pm 2$ \\
\hline $\mathrm{CB}[7]$ & Organic molecule & $8 \pm 3$ \\
\hline $\mathrm{CB}[8]$ & Organic molecule & $8 \pm 3$ \\
\hline Catalytic Antibody & Substrate & $3.5 \pm 1$ \\
\hline Enzyme & Substrate & $3.7 \pm 1.3$ \\
\hline Albumin & Organic molecule & $4.6 \pm 0.9$ \\
\hline Catalytic antibody & Transition state & $6.6 \pm 2.0$ \\
\hline Receptor drug & Drug & $7.3 \pm 1.5$ \\
\hline Antibody & Antigen & $8.1 \pm 2.0$ \\
\hline Enzyme & Inhibitor & $8.6 \pm 4.0$ \\
\hline
\end{tabular}




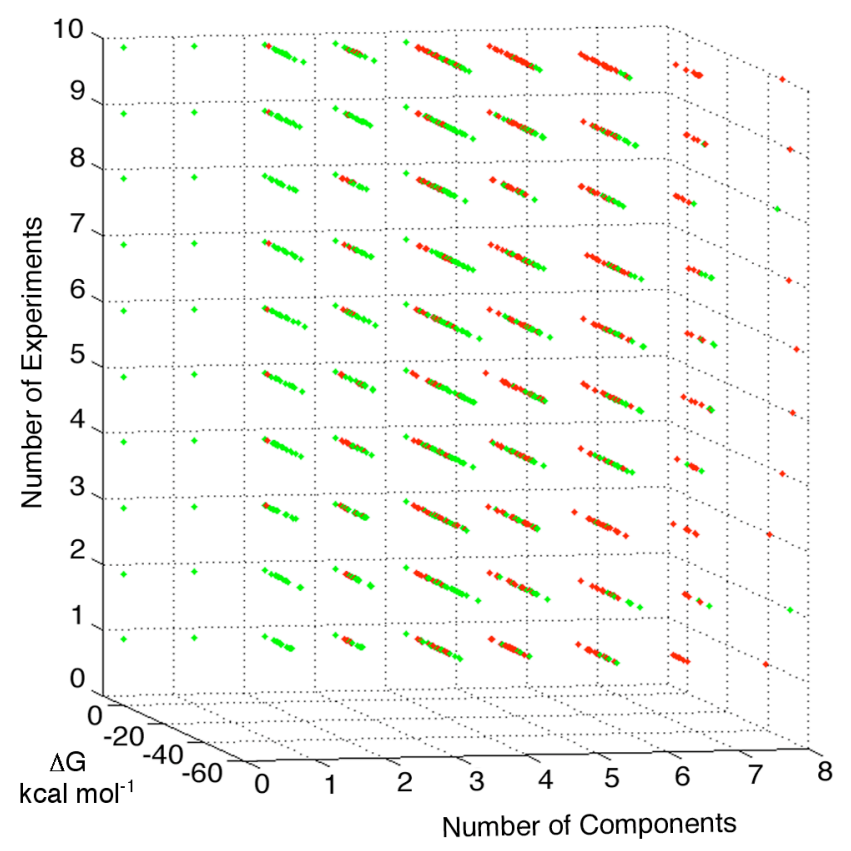

Figure III-12. A stack of ten simulations from randomly generated binding constants showing that only two cases the final state is self-sorted. (states are color coded as follows: self-sorted states, green dots; non self-sorted states, red dots.)

\subsection{Conclusion.}

In summary, we presented compound III-1 - III-8 that form an 8-component self-sorted mixture consisting of III-6•III-1, III-5•III-2, III-7•III-3, and III-8•III-4. We investigated selected pathways by ${ }^{1} \mathrm{H}-\mathrm{NMR}$ and the rest by simulations for the formation of the 8-component mixture. We demonstrated that the presence of a single set of complexes - a self-sorted state - in that 8-component mixture does not have any effect on the intermediate states and the formation of the final state in a step-by-step manner is a path function. Although every path converges to the same final state but their properties are function of the sequence of addition of components. 
The above study demonstrates quite a few aspects that go beyond those of their isolated components are reminiscent to several behaviors observed in biology. First, similar to the molecular networks operating inside the living cells, our designed experimental system is associated with intricate web of molecular recognition events. A hypercube model serves as a network map where a large volume of information is stored in a concise and systematic manner. Second, in many biological processes such as multi-step biosynthesis of proteins, nucleic acids in complex environment in cytoplasm maintaining particular sequences of steps are critical. Although under thermodynamic control - in contrast to Nautre, which operates far from equilibrium under kinetic control - our experimental system is capable of differencing 40320 sequences of addition of components - each of them is characterized as a unique pathway. Third, in the musical chair pathway, the controlled transformation of the mixture into another of quite different composition serves as a minimal model of a signal transduction pathway. Signal transduction events are mostly driven by ordered sequences of enzymatic reactions inside the cell where as transformations in our system are driven by the free energy release upon host-guest complexation. Fourth, just as biological networks, our designed experimental system is also capable of responding to variables like concentration and equilibrium constants. Finally, not only were we able to finish each complete investigation of the system in a fraction of the time by successful application of systems chemistry, we found several concealed trends and pattern in the systems that we did not manifest prior to simulations. These molecules in conjunction with systems chemistry can be used in chemical sensing 
application in complex mixtures and controlling biological catalysis when enzyme are part of the network. 


\section{Chapter 4: Biological Catalysis Controlled by a Synthetic Self-Sorting System.}

\subsection{Introduction.}

In the living organism various regulatory strategies have been employed to control the activity of proteins over space and time. One important strategy that is commonly used in biology is the allosteric regulation where the activities of proteins, including enzymes, are controlled upon binding of small signal molecules to specific sites within the enzymes. ${ }^{145}$ Such allosteric binding events trigger conformational changes that are transmitted to the active site and in turn the activity of the enzyme is either enhanced (positive cooperativity) or reduced (negative cooperativity). For example, hemoglobin, an oxygen transfer protein, shows positive coopetativity where the binding of the first oxygen molecule to one heme group facilitates the binding of oxygen to the other three heme subunits in the same molecule. ${ }^{146}$ Cytidine triphosphate (CTP) acts as an allosteric inhibitor to enzyme aspartate transcarbamoylase (ATCase). Binding of CTP to the regulatory subunit of ATCase shifts the equilibrium towards $\mathrm{T}$ state and therefore decreases the enzymatic activity of ATCase. ${ }^{147-149}$ The key driving force for allosteric regulation and many other events in biology is reversible non-covalent interactions, which inspired others and us to create non-natural systems that exhibit similar regulatory behaviors. ${ }^{150-152}$ 
In this chapter we report a simple strategy to regulate enzymatic activities where we combined an enzyme inhibitor and a non-natural guest into one structure to create a two-faced guest molecule. Our hypothesis was that the presence of the second binding epitope attached to inhibitor molecule would provide the opportunity to regulate biological catalysis upon addition of another receptor, which would sequester the two-face guest from the active site of the enzyme. Although this strategy is uncommon in biology because it requires the evolution of another receptor protein in vivo just to selectivity sequester the inhibitor from the enzyme, it can be a potentially promising for applications in medicinal chemistry. Recently the concept of combination of two drug molecules (hybrid drug) has been used in medicinal chemistry and it turned out that the hybrid drugs often more active. An in vitro assay reveals that a combination of antimalarial drug artemisinin and quinine is more effective against drug-sensitive and drug-resistant malaria than their respective building blocks. ${ }^{153}$ Drug chloroquine was combined with antidepressant imipramine, which counters resistance by inhibiting membrane channel that pumps chloroquine drug's site of action. ${ }^{154}$ This hybrid drug - known as reverse chloroquines - is around 10 times more effective than chloroquine itself. A recent example of hybrid drug combines aspirin with a NO donor. A high dose of aspirin often cause stomach problems. Because nitric oxide can block that toxicity, combination of aspirine and NO donor in one molecule provides the benefit of aspirine without side effects. Surprisingly, aspirin-nitric oxide hybrid drug possesses new anticancer properties, which is uncommon in both pharmacophores. ${ }^{155}$ 
These results as well as our previous experience show that the presence of two binding epitopes in the same molecule gives rise to emergent properties, ${ }^{36}$ that is, the properties of two-face guests are different from individual guest molecules. Herein we explore the strategy of regulation of kinetic events (e.g. biological catalysis) under thermodynamic control using guests IV-1 - IV-5. In this chapter we explore the application of this strategy to control the catalysis of Bovine Carbonic Anhydrase and rationalized a case using Acetylcholinesterase where a potential limitation of this approach predominates.

\subsubsection{Design Aspects of the Chemical Components Used in this Study.}

In medicinal chemistry, it is common to employ reversible inhibitor molecules of enzymes that modulate their enzymatic activities. ${ }^{156-160}$ In some cases it is desirable to turn on or turn off the function of such inhibitors in response to an outside stimulus to regulate the function of the enzyme. The critical requirement for regeneration of enzymatic activity under thermodynamic control is that the association constant $\left(\mathrm{K}_{\mathrm{a}}\right)$ value for non-natural guest•receptor interaction must exceed the enzyme-inhibitor interaction. To design our molecule we settled on benzene sulfonamide, since it is easily functionalizable and a well-studied competitive inhibitor of BCA. ${ }^{161-163}$ Adamantane ammonium ions - as non-natural guest - was our natural choice because it binds to $C B[7]$ with very high affinity $\left(K_{a}=4 \times 10^{12} \mathrm{M}^{-}\right.$ ${ }^{1}$ ), which is approximately $10^{6}$ fold higher than the mean $\mathrm{K}_{\mathrm{a}}$ of sulfonamides to BCA. $^{31}$ Based on our design we synthesized $\mathbf{I V - 1}$ and envisioned that addition of 
$\mathrm{CB}[7]$ to the enzyme-inhibitor complex would result in the sequestration of the inhibitor as the $\mathrm{CB}[7] \cdot$ inhibitor complex thus releasing free $\mathrm{BCA}$ and regenerating catalysis (Scheme IV-1). Of critical importance in the selection of the enzyme/inhibitor pairs were: 1) each enzyme should have straightforward and wellstudied assays, 2) the cognate inhibitor should be easily functionalizable, and 3) the inhibitor should preserve its function even after derivatization.

Scheme IV-1. Thermodynamic Shuttling of IV-1 between BCA and CB[7].

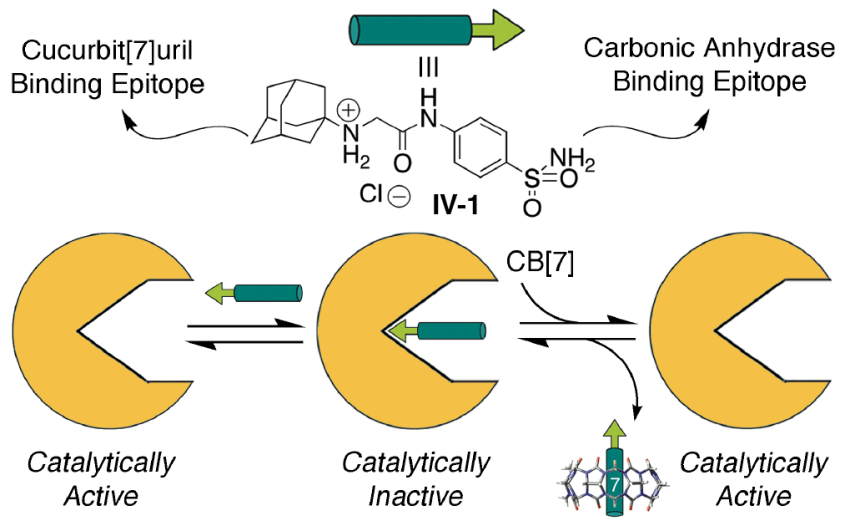

\subsection{Results and Discussion.}

This results and discussion section is organized as follows. First, we introduce thirteen compounds IV-1 - IV-13 used in this study (Chart IV-1). Then we introduced the assays that we used to monitor the activity of the enzymes. Next, we demonstrate how $\mathrm{CB}[7]$ can regenerate enzymatic activity of Bovine Carbonic Anhydrase (BCA) by sequestering compound IV-1 and IV-2 from its active site. Subsequently, we discuss in detail the mechanism of the shuttling of IV-1 and IV-2 
between $\mathrm{CB}[7]$ and $\mathrm{BCA}$ based on results from fluorophore displacement assays. In the final section of this chapter we discuss the pitfalls encounter when we attempted to use two-faced guest molecules to control the enzymatic behavior of AChE.

Chart IV-1. Compounds Used in this Study.

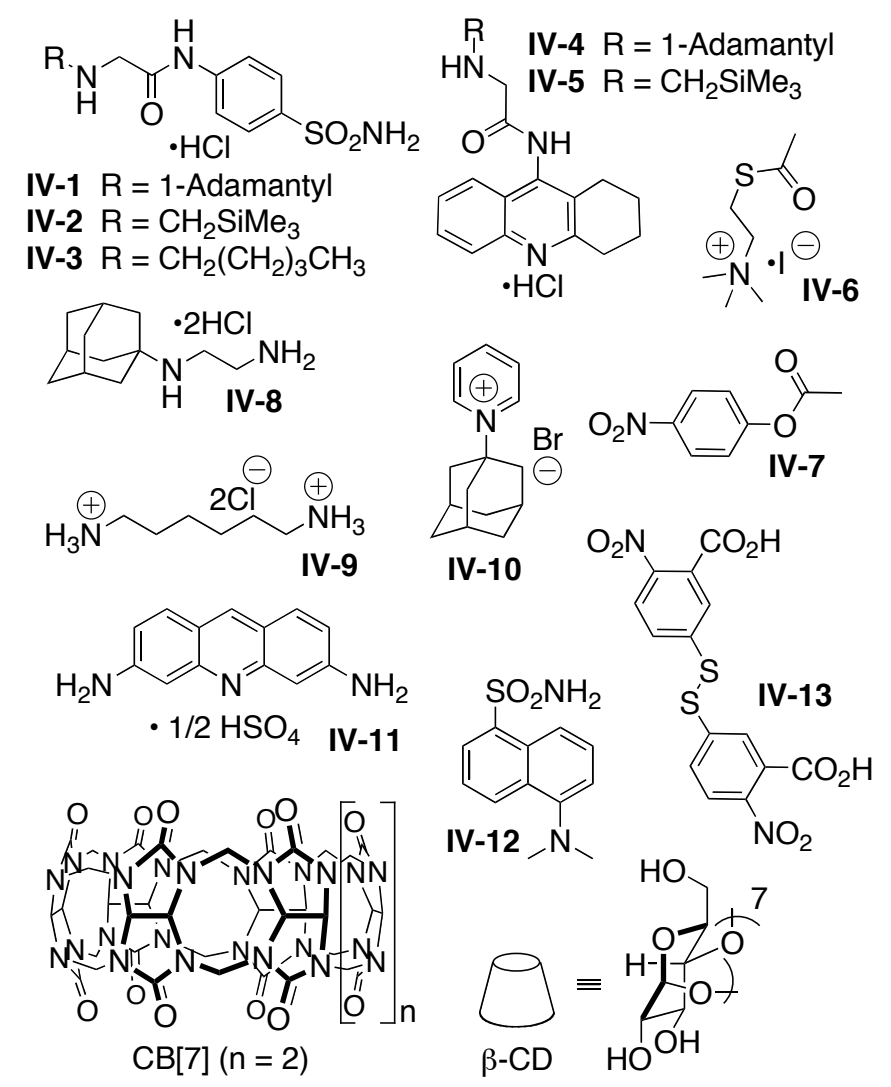

\subsubsection{Synthesis of Guests IV-1 - IV-5.}

Scheme IV-2 shows the synthesis of two-faced guests IV-1 - IV-5. Compound IV-16 was synthesized in 83\% yield from IV-14 and IV-15. Compound 
IV-16 was reacted with the appropriate amine under Finkelstein reaction conditions to deliver amines IV-1 - IV-3 in $62-73 \%$ yield. The hydrochloric acid salt of IV-1 - IV-3 was prepared by dissolving the amine in $\mathrm{EtOH}$ and passing $\mathrm{HCl}$ gas through the solution. Compounds IV-4 and IV-5 were synthesized in two steps from IV-17 in a similar manner. First, the acylation of IV-14 with IV-17 gave compound IV-18 in $42 \%$ yield. Subsequently, IV-18 was alkylated with adamantane amine or $\mathrm{H}_{2} \mathrm{NCH}_{2} \mathrm{SiMe}_{3}$ to give amines IV-4 and IV-5 in 62 and $67 \%$ yield. The hydrochloric acid salt of IV-4 and IV-5 was prepared by dissolving the amine in EtOH and passing $\mathrm{HCl}$ gas through the solution.

IV-14

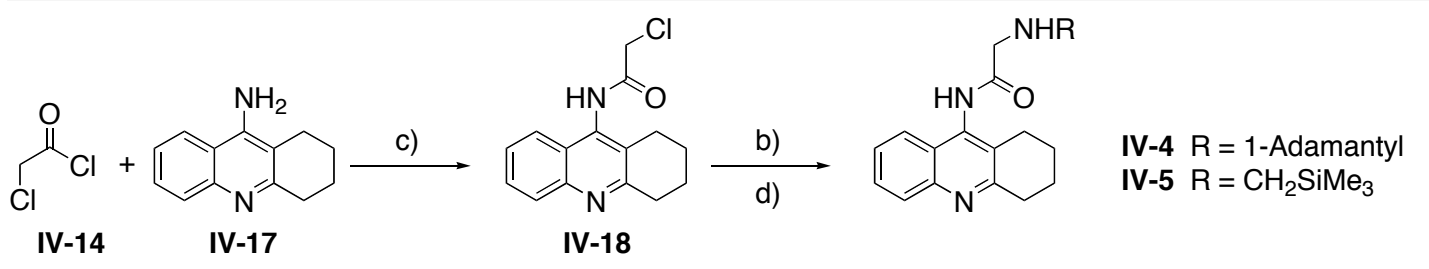

Scheme IV-2. Synthesis of IV-1 - IV-5. Conditions: a) $\mathrm{K}_{2} \mathrm{CO}_{3}$ or $\mathrm{Et}_{3} \mathrm{~N}$, THF, reflux, b) $\mathrm{RNH}_{2}, \mathrm{~K}_{2} \mathrm{CO}_{3}, \mathrm{KI}$, anh. THF, reflux, c) $140{ }^{\circ} \mathrm{C}$, neat, d) $\mathrm{HCl}(\mathrm{g}), \mathrm{EtOH}$.

\subsubsection{Chemical Reactions Used to Monitor Enzymatic Activities.}

We used two straightforward colorimetric assays that allowed us to quantify the catalytic activity of $\mathrm{BCA}$ and $\mathrm{AChE} . \mathrm{BCA}$ is an esterase that catalyzes the hydrolysis of IV-7 to form yellow-colored IV-19. Therefore when the inhibitor 
molecule occupies the active site of the enzyme, formation of IV-19 is not observed. Consequently, the rate of hydrolysis can be monitored by measuring the absorbance at $400 \mathrm{~nm}$ by UV/Vis spectroscopy (Scheme IV-3a). ${ }^{164-166}$ The enzymatic activity of AChE can be measured by the Ellman assay. The Ellman assay relies on the AChE catalyzed hydrolysis of IV-6 to IV-20 and acetate. Thiolate IV-20 reacts rapidly with IV-13 to generate IV-22, which has a yellow color $\left(\lambda_{\max }=412 \mathrm{~nm}\right)$. The rate of enzyme hydrolysis, therefore can be monitored by measuring absorbance at $412 \mathrm{~nm}$ using UV/Vis spectroscopy (Scheme IV-3b). ${ }^{167}$

Scheme IV-3. Chemical Reactions Used to Monitor Enzymatic Activities.

a)
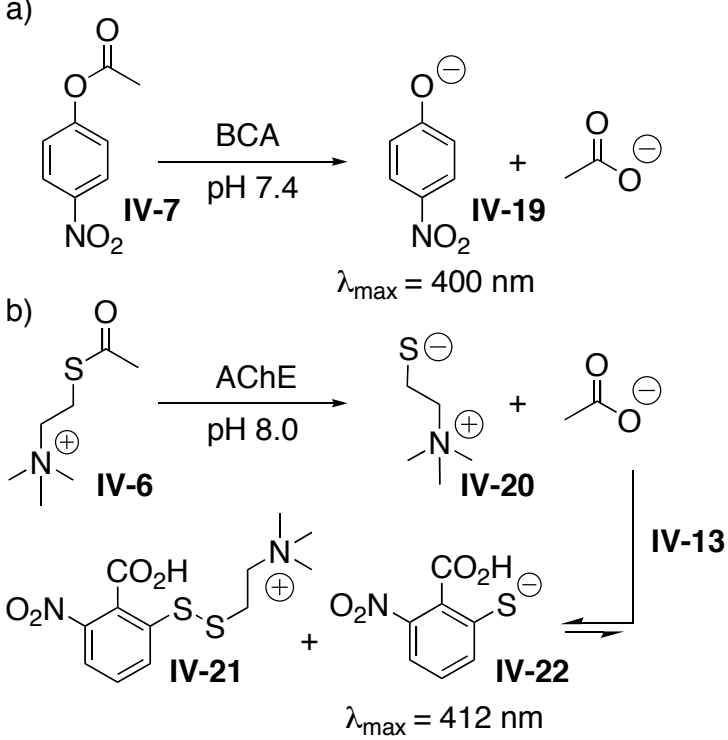


\subsubsection{Regulation of Biological Catalysis of Bovine Carbonic Anhydrase.}

As a first test of our design strategy, we monitored the enzymatic hydrolysis of IV-7 by BCA before and after the addition of IV-1. A shut down of the hydrolysis of IV-7 was observed, which indicates that IV-1 inhibits the enzymatic activity of BCA due to formation of BCA $-\mathbf{I V - 1}$ (Step 1 and 2; Figure IV-1b). In the next step, when we monitored the enzymatic hydrolysis of IV-7 after addition of $\mathrm{CB}[7]$ to a solution of $\mathrm{BCA} \cdot \mathbf{I V}-\mathbf{1}$. We observed $44 \%$ regeneration of the initial enzymatic activity (Step 3; Figure IV-1b). We expected complete a regeneration under thermodynamic control based on our design and only partial regeneration of enzymatic activity after addition of $\mathrm{CB}[7]$ was somewhat surprising.

Finally, we expected that addition of IV-8 to the resulting mixture of BCA, IV-1 and CB[7] trigger a new self-sorted state where IV-8 form complex with CB[7] freeing guest IV-1, which in turn result in the inhibition of the hydrolysis of IV-7. ${ }^{168}$ When we performed the experiment, we observed that $42 \%$ catalytic activity (Step 4 ; Figure IV-1b). This result indicates that addition of IV-8 failed to release IV-1 from $\mathrm{CB}[7]$. We rationalize this observation based on literature precedent that adamantane amine has slow dissociation rate constant $\left(\mathrm{k}_{\text {off }}=2.4 \times 10^{-5} \mathrm{~s}^{-1}\right)$ from $\mathrm{CB}[7] .{ }^{36}$ Although IV-8 has higher affinity for CB[7] than IV-1, due to the slow rate of dissociation of IV-1 was not released from the cavity of $\mathrm{CB}[7]$ and therefore did not turn off enzymatic activity. Under thermodynamic consideration IV-1 is suitable for shuttling between $\mathrm{BCA}$ and $\mathrm{CB}$ [7], experimental implementation of this design was not possible as the system operates under kinetic control due to slow $\mathrm{k}_{\text {off }}$ of IV-1 from $\mathrm{CB}[7]$. 
The partial regeneration of enzymatic activity using IV-1 was intuitively promising and inspired us to modify our design to improve the performance. We designed new two-faced guest IV-2 bearing (trimethylsilyl)methyl amine instead of adamantane amine. The design of IV-2 was based on our experience and precedent from literature that (trimethylsilyl)methyl amine has substantial affinity $\left(\mathrm{K}_{\mathrm{a}}=8.98 \times\right.$ $10^{8} \mathrm{M}^{-1}$ ) toward $\mathrm{CB}[7]$, also the complex has a fast dissociation rate constant. A colorimetric assay indicated that $\mathbf{I V - 2}$ was capable of inhibiting the enzymatic activity of BCA (Step 1 and 2; Figure IV-1a and IV-1b). When we added CB[7] to a solution of $\mathrm{BCA} \cdot \mathbf{I V - 2}$ we observed maximum $83 \%$ regeneration of the initial enzymatic activity (Step 3; Figure IV-1a and IV-1b). Upon addition of IV-8 to the resulting mixture of BCA, IV-2 and CB[7], we successfully turned off the hydrolysis of IV-7 (Step 4; Figure IV-1a and IV-1b). Addition of compound IV-8 triggers a new self-sorted state under thermodynamic control where IV-8 binds to CB[7] by releasing IV-2 which in turn inhibits BCA. We were able to continue such alternate deactivation and regeneration of enzyme activity by adding CB[7] and IV-8 in an alternating sequence (Step $5-8$; Figure IV-1a and IV-1b). An explanation for the steady decrease in the relative activity of BCA over the successive cycles of deactivation and regeneration of enzymatic activity (Figure IV-1a) was at first poorly understood. Later, we rationalized this behavior using fluorescence spectroscopy. We demonstrated that only partial instead of complete regeneration of the enzymatic activity of $\mathrm{BCA}$ was observed due to inhibition by $\mathrm{CB}[7] \cdot \mathbf{I V}-\mathbf{1}$ after $\mathrm{CB}[7]$ sequesters IV-1 from BCA to form a ternary complex BCA $\mathbf{I V}-\mathbf{1} \cdot \mathrm{CB}[7]$. CB[7]・IV-2 has weaker inhibition capability that $\mathrm{CB}[7] \cdot \mathbf{I V}-\mathbf{1}$, hence we observed higher regenerated 
enzymatic activity. Formation of $\mathrm{BCA} \cdot \mathbf{I V}-\mathbf{2} \cdot \mathrm{CB}[7]$ is responsible for incomplete regeneration after addition of $\mathrm{CB}[7]$ and steady decrease in the enzymatic activity over the successive cycles.

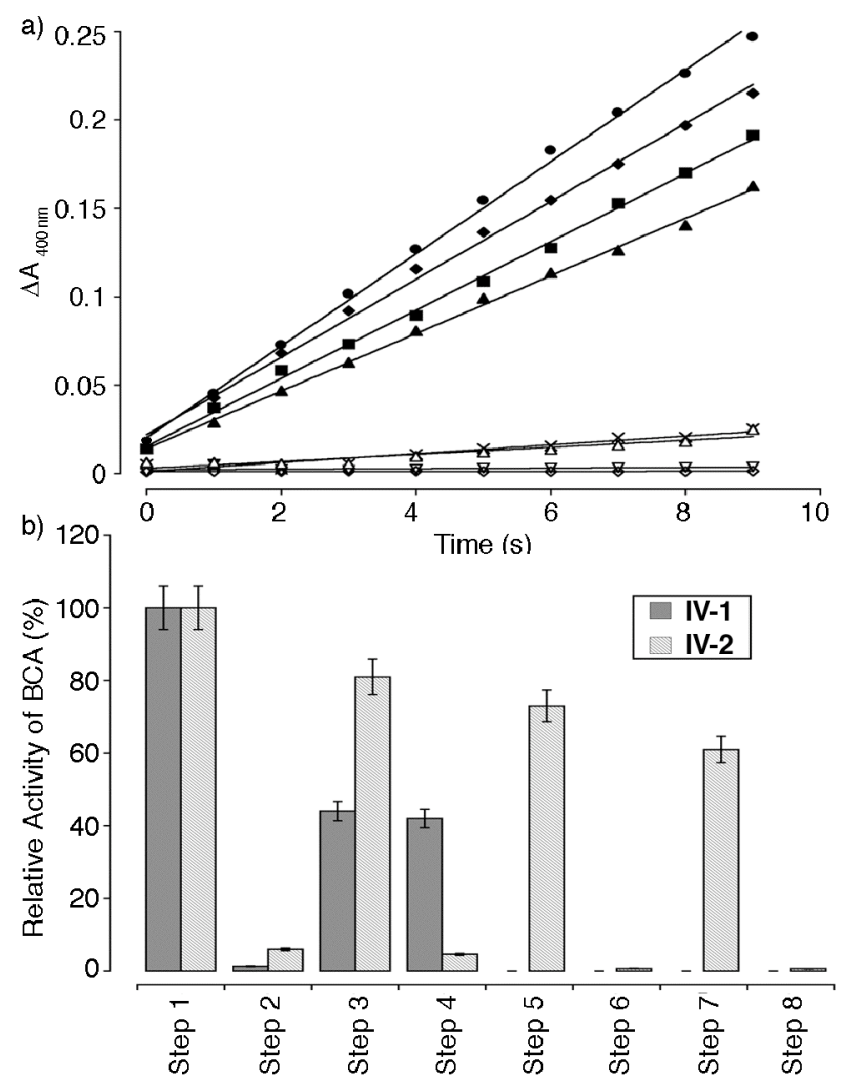

Figure IV-1. a) A plot of change in absorbance versus time for IV-2: Step $1(\mathbf{\bullet})$, Step $2(\times)$; Step $3(\bullet)$; Step $4(\triangle)$; Step $5(\mathbb{\square})$; Step $6(\diamond)$; Step $7(\mathbf{\Lambda})$; and Step 8 $(\nabla)$. b) A plot of relative activity of BCA versus number of steps.

If the swapping of guests from the active site of $\mathrm{BCA}$ to $\mathrm{CB}[7]$ was driven by the fact that IV-1 and IV-2 have a higher affinity towards $\mathrm{CB}[7]$ than BCA, we proposed that the enzymatic activity will not be regenerated when the non natural 
guest - receptor interaction is weaker than sulfonamide - BCA interaction. We used $\beta$-CD since it is known to have a modest affinity towards adamantanes $\left(\mathrm{K}_{\mathrm{a}}=10^{4} \mathrm{M}^{-}\right.$ $\left.{ }^{1}\right) .{ }^{140}$ As predicted, we were unable to regenerate the enzymatic activity by removing IV-1 from the active site of BCA $\mathbf{I V}-\mathbf{1}$ using $\beta$-CD. As a second control experiment, we designed compound IV-3 where we replaced the higher affinity CB[7] binding groups adamantane amine and (trimethylsilyl)methyl amine by a lower affinity $\mathrm{CB}[7]$ binding group hexylamine. We did not observe any regeneration of enzymatic activity after addition of $\mathrm{CB}[7]$ to a solution containing $\mathrm{BCA} \cdot \mathbf{I V}-\mathbf{3}$. These control experiments strongly suggest that the shuttling of guests operates under thermodynamic control based on differences in the value of binding constants of the appendages to their cognate receptors.

The UV/Vis assay described above serves as an indirect evidence of guest exchange between receptors. A direct evidence of guest shuttling can be obtained from ${ }^{1} \mathrm{H}$ NMR spectroscopy, which can track the location of the TMS group present in IV-2. Figure IV-2a shows the ${ }^{1} \mathrm{H}$ NMR spectrum of BCA in the region from 0.5 to $-1 \mathrm{ppm}$. The appearance of a new peak at $-0.27 \mathrm{ppm}$ upon addition of IV-2 to BCA is indicative of the formation of the BCA $\bullet I V-2$ complex (Figure IV-2b). After addition of excess $\mathrm{CB}[7]$ the resonance for BCA•IV-2 disappeared completely and only the resonance for $\mathrm{CB}[7] \cdot \mathbf{I V}-2$ was observed (Figure IV-2d). ${ }^{1} \mathrm{H}$ NMR provides evidences in support of the swapping of IV-2 between BCA and CB[7] and are consistent with result obtained from the colorimetric assay. 

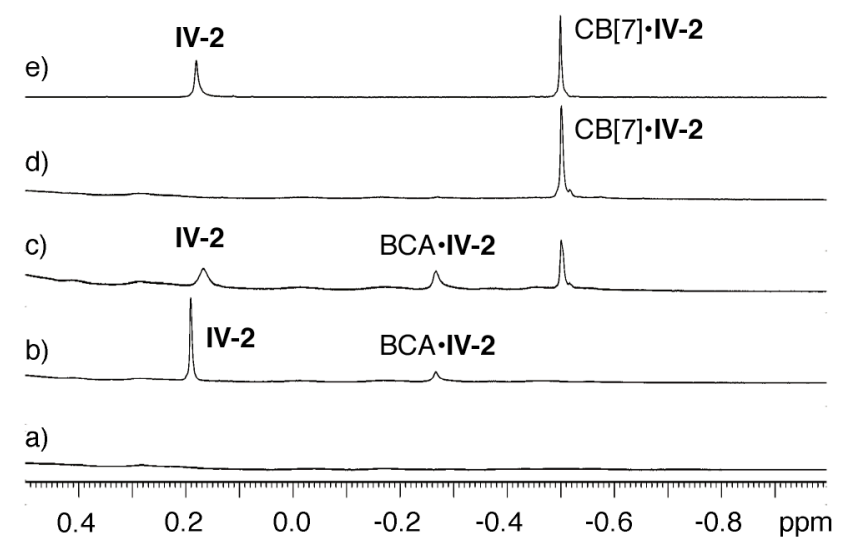

Figure IV-2. ${ }^{1} \mathrm{H}$ NMR spectra recorded for $\left(400 \mathrm{MHz}, 30 \mathrm{mM} \mathrm{NaD}{ }_{2} \mathrm{PO}_{4}\right.$ Buffer, pD 7.3, $298 \mathrm{~K}$ ): a) BCA (1 eq.), b) BCA (1 eq.) and IV-2 (1.76 eq.), c) BCA (1 eq.), IV-2 (1.76 eq.) and CB[7] (0.5 eq.), d) BCA (1 eq.), IV-2 (1.76 eq.) and CB[7] (2.0 eq.), and e) IV-2 (2 eq.) and CB[7] (1 eq.).

\subsubsection{Measurement of Association Constant.}

As described above, the successful alternate turn on and off sequence of the biological catalysis of BCA using IV-2, the results obtained from ${ }^{1} \mathrm{H} \mathrm{NMR}$, and control experiments suggest the shuttling of guests operates under thermodynamic control. To provide strong evidence for this interpretation, we decided to measure or estimated the value of $\mathrm{K}_{\mathrm{a}}$ of the various receptor $\bullet$ guest pairs.

\subsubsection{Measurement of the $\mathrm{K}_{\mathrm{a}}$ of the Inhibitor to $\mathrm{CB}[7]$.}

The association constant of IV-1 and IV-2 to CB[7] were measured by competition with appropriate guests of known values of $\mathrm{K}_{\mathrm{a}}$ by using ${ }^{1} \mathrm{H}$ NMR competition 
experiments. All binding experiments were performed in $50 \mathrm{mM} \mathrm{NaO} \mathrm{CCD}_{3}$ buffered $\mathrm{D}_{2} \mathrm{O}(\mathrm{pD} 4.75)$ at $25{ }^{\circ} \mathrm{C} .{ }^{31}$ The association constants of IV-1 $\left(\mathrm{K}_{\mathrm{a}}=4.13 \pm\right.$ $\left.0.935 \times 10^{12} \mathrm{M}^{-1}\right)$ and IV-2 $\left(\mathrm{K}_{\mathrm{a}}=2.5 \times 10^{8} \mathrm{M}^{-1}\right)$ were measured by competition with IV-10 and IV-9 respectively for a limiting quantity of CB[7] (Figure IV-9). ${ }^{169}$ The value of $\mathrm{K}_{\mathrm{a}}$ for $\mathrm{CB}[7] \cdot \mathbf{I V}-\mathbf{3}$ was estimated as $10^{6} \mathrm{M}^{-1}$ by extrapolation of results by us and Mock and co-workers. ${ }^{32,33,170}$

\subsubsection{Evidence of Shuttling from Fluorescence Spectroscopy.}

We used a competitive fluorescence assay as an alternate method to understand the mechanism of guest exchange process. Unbound IV-12 has a low quantum yield in water and increases markedly (at $460 \mathrm{~nm}$ ) when it forms a 1:1 complex in the non-polar and hydrophobic environment inside BCA. Addition of IV$\mathbf{1}$ or IV-2 to a solution containing BCA-IV-12 resulted in a decrease in fluorescence intensity at $460 \mathrm{~nm}$, which indicates the displacement of IV-12 from the active site of BCA by IV-1 or IV-2 (Scheme IV-4). In this manner fluorogenic probe IV-12 signals whether the two-faced guests is bound to the enzyme or not. ${ }^{162,171,172}$ Interestingly, when $\mathrm{CB}[7]$ was added to that solution, we observed an increase in fluorescence intensity at $460 \mathrm{~nm}$. This observation indicates the formation of a new self-sorted state consisting of BCA $\mathbf{I V - 1 2}$ and CB[7] $\cdot$ IV-2 in solution. Finally, a decrease in fluorescence intensity at $460 \mathrm{~nm}$ was observed after addition of IV-8, indicating another complete reorganization to form a new self-sorted state consisting of $\mathrm{CB}[7] \cdot \mathbf{I V}-\mathbf{8}$ and $\mathrm{BCA} \cdot \mathbf{I V}-\mathbf{2}$. The alternate increase and decrease of fluorescence 
intensity at $460 \mathrm{~nm}$ supports our interpretation that the on / off of biological catalysis is due to binding and dissociation of IV-2 from the active site of BCA triggered by the presence of $\mathrm{CB}[7]$.

Scheme IV-4. Schematic Representation of the Fluorophore Displacement Assay.

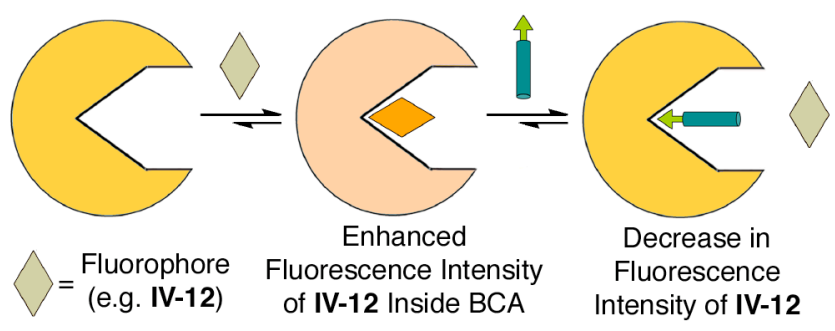

\subsubsection{Measurement of the Association Constant $\left(\mathrm{K}_{\mathrm{a}}\right)$ of Inhibitors IV-1 - IV-3 to the BCA.}

We directly measured the value of $\mathrm{K}_{\mathrm{a}}$ for BCA $\bullet \mathbf{I V - 1 2}$ by monitoring the increase in fluorescence intensity at $460 \mathrm{~nm}$ of BCA with the concomitant increase of the concentration of IV-12 and fitting the data by nonlinear least-squares analysis of a 1:1 binding model (Figure IV-13 and IV-14). The binding constant values of BCA•inhibitor were measured by a competitive fluorophore displacement assay ${ }^{173}$ where we monitored the decrease in fluorescence intensity at $460 \mathrm{~nm}$ of BCA $\mathbf{I V}-\mathbf{1 2}$ with the concomitant increase of the concentration of IV-1 - IV-3. The values of $\mathrm{K}_{\mathrm{a}}$ of IV-1 - IV-3 were calculated by fitting equation IV-1 to decrease in fluorescence intensity. (Figure IV-3 and IV-15). ${ }^{173}$ 


$$
\frac{F-F_{\text {Min }}}{F_{\text {Max }}-F_{\text {Min }}}=\frac{1}{1+\left(\frac{K^{d}{ }_{D N S A}}{[D N S A]}\right)\left(1+\frac{[\operatorname{lnh}]}{K^{d_{I n h}}}\right)}
$$

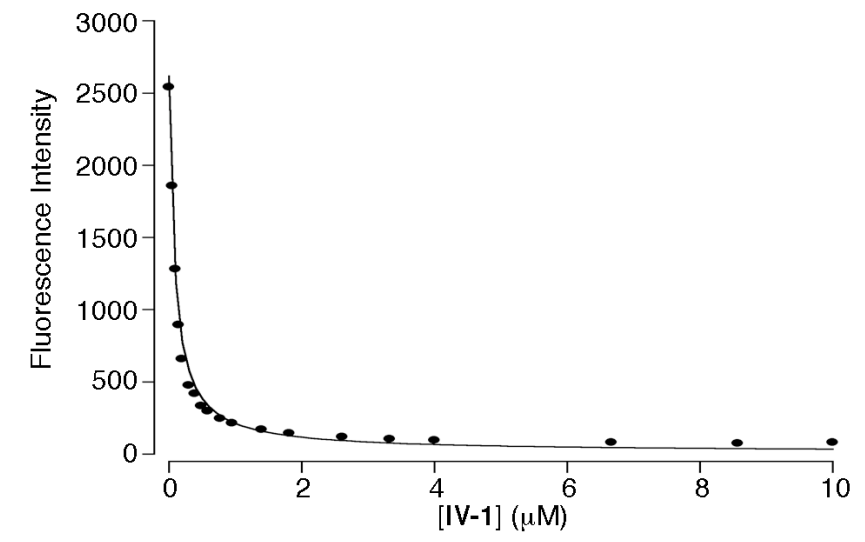

Figure IV-3. Plot of fluorescence intensity versus $[\mathbf{I V}-1]$ in the titration of BCA $\bullet$ IV12 with IV-1. Concentration of BCA (67.5 nM), IV-12 $(2 \mu \mathrm{M})$, and IV-1 (50 nM - 10 $\mu \mathrm{M})$. All measurements were done in $20 \mathrm{mM} \mathrm{NaH}_{2} \mathrm{PO}_{4}$ Buffer, pH 7.3, $298 \mathrm{~K}$. 
Table IV-1. Thermodynamic and Kinetic Parameters for BCA Inhibitor Interactions.

\begin{tabular}{|c|c|c|c|c|c|}
\hline & \multicolumn{5}{|c|}{ Bovine Carbonic Anhydrase } \\
\hline & $\mathrm{K}_{\mathrm{a}}\left(\mathrm{M}^{-1}\right)$ & $\begin{array}{l}\Delta \mathrm{G}(\mathrm{kcal} \\
\left.\mathrm{mol}^{-1}\right)\end{array}$ & $\mathrm{k}_{\mathrm{on}}\left(\mathrm{M}^{-1} \mathrm{~s}^{-1}\right)$ & $\mathrm{k}_{\mathrm{off}}\left(\mathrm{s}^{-1}\right)$ & $\begin{array}{l}\mathrm{t}_{1 / 2}(\mathrm{~s}) \text { of } \\
\text { dissociation }\end{array}$ \\
\hline IV-12 & $\begin{array}{l}(3.87 \pm 0.34) \\
\times 10^{6}\end{array}$ & -8.96 & $4.7 \times 10^{5}$ & $\begin{array}{l}(1.20 \pm 0.10) \\
\times 10^{-1}\end{array}$ & 5.8 \\
\hline IV-2 & $\begin{array}{l}(2.73 \pm 0.24) \\
\times 10^{7}\end{array}$ & -10.11 & $2.8 \times 10^{5}$ & $\begin{array}{l}(1.40 \pm 0.01) \\
\times 10^{-2}\end{array}$ & 49.5 \\
\hline IV-1 & $\begin{array}{l}(1.08 \pm 0.10) \\
\times 10^{8}\end{array}$ & -10.92 & $5.1 \times 10^{5}$ & $\begin{array}{l}(4.30 \pm 0.01) \\
\times 10^{-3}\end{array}$ & 161.1 \\
\hline $\begin{array}{l}\text { CB[7]• } \\
\text { IV-2 }\end{array}$ & $\begin{array}{l}(2.56 \pm 0.21) \\
\times 10^{5}\end{array}$ & -7.36 & $6.5 \times 10^{4}$ & $\begin{array}{l}(2.70 \pm 0.13) \\
\times 10^{-1}\end{array}$ & 2.6 \\
\hline $\begin{array}{l}\text { CB[7]• } \\
\text { IV-1 }\end{array}$ & $\begin{array}{l}(9.14 \pm 0.77) \\
\times 10^{5}\end{array}$ & -8.10 & $3.1 \times 10^{4}$ & $\begin{array}{l}(4.81 \pm 0.58) \\
\times 10^{-2}\end{array}$ & 14.3 \\
\hline
\end{tabular}

\subsubsection{Kinetic Effect in Enzyme Inhibitor Binding.}

While we were carrying out the fluorophore displacement assay, we observed interesting dynamic behavior in the enzyme-inhibitor binding event. For example, when $\mathrm{CB}[7]$ was added to a solution of $\mathrm{BCA} \cdot \mathbf{I V}-\mathbf{1}$ and $\mathbf{I V - 1 2}$ (also to a solution of BCA $\cdot \mathbf{I V - 2}$ and IV-12) we observed an initial increase followed by a decrease in the fluorescence intensity (Figure IV-4). An identical decay pattern was observed (Figure IV-27) after adding $\mathrm{CB}[7] \cdot \mathbf{I V}-\mathbf{1}$ to a solution of $\mathrm{BCA} \cdot \mathbf{I V}-\mathbf{1 2}$ indicating that $\mathrm{CB}[7] \cdot \mathbf{I V}-\mathbf{1}$ is also capable of interacting with BCA. We anticipated that such 
behavior might influence the yield of regenerated BCA and therefore decided to further explore the kinetic aspect of the shuttling process.

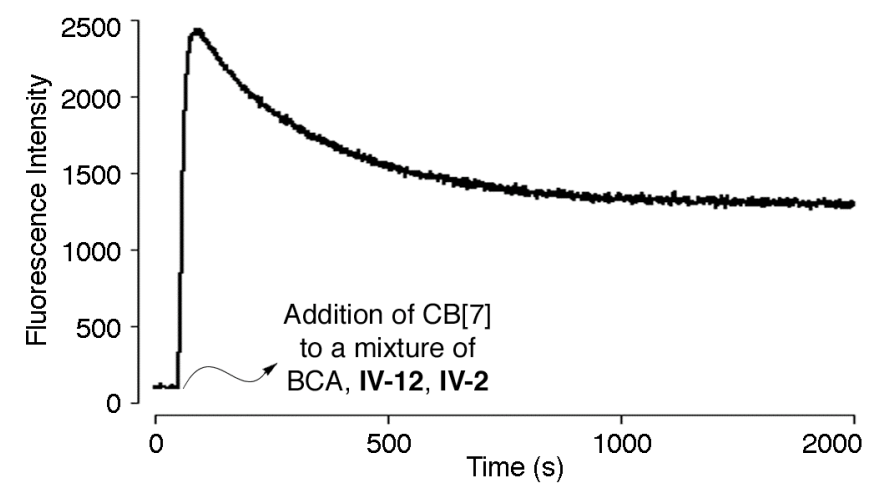

Figure IV-4. Plot of fluorescence intensity versus time after addition of CB[7] (50 $\mu \mathrm{M})$ to a mixture of IV-12 $(2 \mu \mathrm{M})$, IV-1 $(20 \mu \mathrm{M})$ and BCA $(80 \mathrm{nM})$. All measurements were done in $20 \mathrm{mM} \mathrm{NaH} \mathrm{PO}_{4}$ buffer, $\mathrm{pH}$ 7.3, $298 \mathrm{~K}$.

4.2.9 Determination of the Values of $\mathrm{k}_{\text {on }}$ and $\mathrm{k}_{\text {off }}$ of the inhibitor from BCA.

When we attempted to measure the value of dissociation rate constant $\left(\mathrm{k}_{\text {off }}\right)$ of IV-12 from BCA, we observed that it initially increases with concomitant increase in the concentration of IV-1 and finally reach to a saturation value (Figure IV-23). The dependence of dissociation rate constant of IV-12 from BCA on the concentration of IV-1 can be explained by using equation IV-2. In equation IV-2, $\mathrm{k}_{1}$ and $\mathrm{k}_{-1}$ are the association and dissociation rate constants of IV-12 to BCA. Similarly, $\mathrm{k}_{2}$ and $\mathrm{k}_{-2}$ are the association and dissociation rate constants of IV-1 to BCA. 
$\mathrm{BCA} \cdot \mathbf{I V}-\mathbf{1 2}+\mathbf{I V}-\mathbf{1} \underset{\mathrm{k}_{1}}{\stackrel{\mathrm{k}_{-1}}{\rightleftharpoons}} \mathrm{BCA}+\mathbf{I V}-\mathbf{1 2}+\mathbf{I V}-\mathbf{1} \underset{\mathrm{k}_{-2}}{\stackrel{\mathrm{k}_{2}}{\rightleftharpoons}}$ BCA $\bullet \mathbf{I V}-\mathbf{1}+\mathbf{I V}-\mathbf{1 2}$

At lower concentration of $\mathbf{I V - 1}$, addition of $\mathbf{I V - 1}$ to $\mathrm{BCA}$ is the ratedetermining step; hence the aparant rate constant $\left(\mathrm{k}_{\mathrm{obs}}\right)$ is a function of both concentration of IV-1 and IV-12. We measured the value dissociation rate constant of IV-12 from BCA under a pseudo first-order condition by adding IV-1 in sufficient excess to ensure that step 1 is the rate-determining step and fitting the decrease in fluorescence intensity at $460 \mathrm{~nm}$ to an exponential decay equation (Figure IV-24). In a similar manner, we calculated the value of dissociation rate constant of IV-1 and IV-2 from BCA by adding excess IV-12 to BCA $\bullet \mathbf{I V}-\mathbf{1}$ or BCA $\bullet \mathbf{I V}-\mathbf{2}$ to ensure that dissociation of IV-1 and IV-2 is the rate-determining step (Figure IV-18 - IV-22).

Somewhat surprisingly, when we measured the $\mathrm{k}_{\text {off }}$ of $\mathbf{I V - 1}$ in presence of $\mathrm{CB}[7]$ we found it is 11 times faster than when measured in absence of $\mathrm{CB}$ [7] (Figure IV-5a and IV-5b). Our interpretation is that $\mathrm{CB}[7]$ interacted with the adamantane amine binding epitope of $\mathrm{BCA} \bullet \mathbf{I V}-\mathbf{1}$ and catalyzes its dissociation from the active site of the enzyme (Scheme IV-5). In the same manner, addition of CB[7] to a mixture of BCA $\mathbf{I V - 2}$ and IV-12 displayed a 19-fold enhancement of $\mathrm{k}_{\text {off }}$ of IV-2 from BCA (Figure IV-25 and IV-26). 


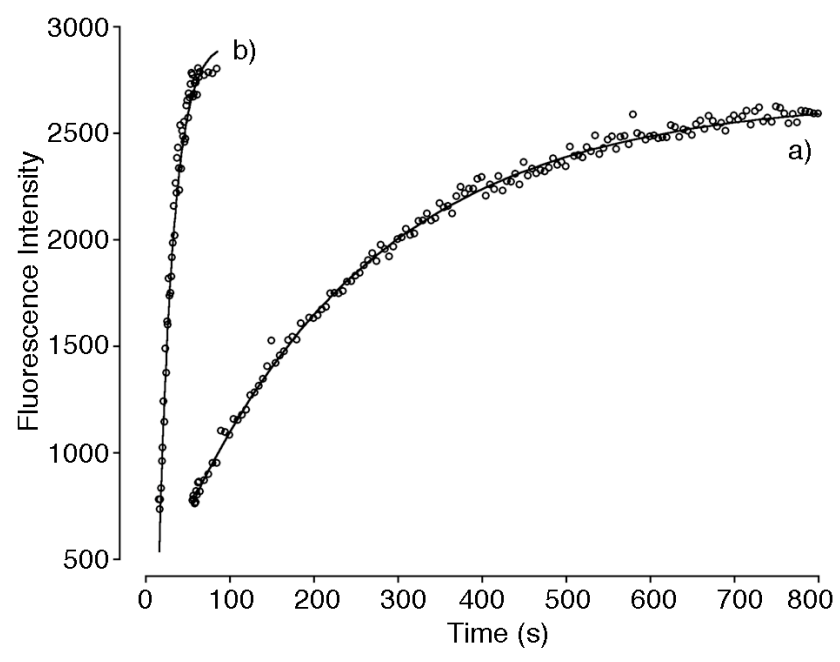

Figure IV-5. Plot of fluorescence intensity versus time: a) Addition of IV-12 to a solution of BCA and IV-1. Final concentration of BCA, IV-12, and IV-1 are $76 \mathrm{~nm}$, $98 \mu \mathrm{M}$ and $1.5 \mu \mathrm{M}$. b) Addition of $\mathrm{CB}[7]$ to a solution containing BCA, IV-12, and IV-1. Final concentrations of BCA, IV-12, IV-2 and CB[7] are $75 \mathrm{nM}, 25 \mu \mathrm{M}, 18$ $\mu \mathrm{M}$ and $50 \mu \mathrm{M}$, respectively. All experiments were done in $20 \mathrm{mM} \mathrm{NaH} \mathrm{PO}_{4} \mathrm{Buffer}$ $\mathrm{pH} 7.3,298 \mathrm{~K}$.

Scheme IV-5. Proposed Mechanism of CB[7] Catalyzed Dissociation of IV-1 and IV-2 from BCA.

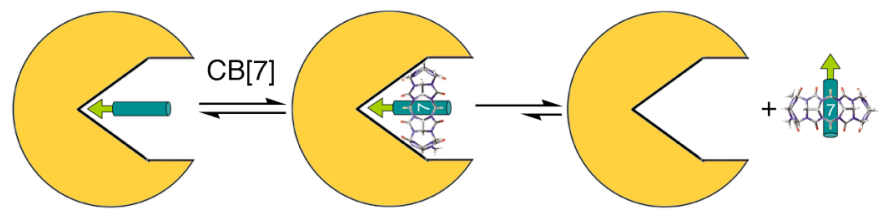




\subsubsection{Formation of Ternary Complexes BCA $・ \mathbf{I V}-\mathbf{1} \cdot \mathrm{CB}[7]$ and $\mathrm{BCA} \bullet \mathbf{I V}-$ $2 \cdot \mathrm{CB}[7]$}

We observed a slow decay of fluorescence intensity at $460 \mathrm{~nm}$ when we added a 1:1 mixture of $\mathrm{CB}[7] \cdot \mathbf{I V}-\mathbf{1}$ to a mixture of $\mathrm{BCA} \cdot \mathbf{I V}-\mathbf{1 2}$ as opposed to a sharp decrease fluorescence intensity when same amount of IV-1 was added to a same concentration of BCA $\mathbf{I V - 1 2}$. We anticipated that such decrease in fluorescence intensity is due to formation of ternary complex BCA $\bullet \mathbf{I V}-1 \cdot \mathrm{CB}[7]$ and decided to measure the $\mathrm{K}_{\mathrm{a}}$ of $\mathrm{CB}[7] \cdot \mathrm{IV}-\mathbf{1}$ to $\mathrm{BCA}$. We used the same technique to measure the binding constant as before. Complex $\mathrm{CB}[7] \cdot \mathbf{I V}-\mathbf{1}$ possesses a slower association rate constant and faster dissociation rate constant, therefore weaker inhibition capability than IV-1 (Figure IV-16).

Based on our measurement of the kinetic and thermodynamic parameters in the $\mathrm{BCA} \cdot$ inhibitor interaction we can explain the pattern of fluorescence intensity show in Figure IV-4. When CB[7] was added to the mixture of BCA, IV-12, and IV$\mathbf{1}$ it forms BCA $\bullet \mathbf{I V}-1 \cdot \mathrm{CB}[7]$ which undergo a rapid dissociation resulting in a steep rise in fluorescence intensity. The exponential decay of the fluorescent intensity after reaching the maxima can be explained as the slow association of $\mathrm{CB}[7] \cdot \mathbf{I V}-\mathbf{1}$ to $\mathrm{BCA}$ to form $\mathrm{BCA} \cdot \mathbf{I V}-1 \cdot \mathrm{CB}[7]$. The association rate constant of $\mathrm{CB}[7] \cdot \mathbf{I V}-2$ was measured in a similar manner but we could not explain the origin of the difference in inhibition capabilities of CB[7]•IV-1 and CB[7]•IV-2 (Figure IV-17). The results of the fluorescence experiments demonstrate that $\mathrm{CB}[7] \cdot \mathbf{I V}-\mathbf{2}$ has weaker inhibition capability than $\mathrm{CB}[7] \cdot \mathbf{I V}-\mathbf{1}$. Therefore we observed only $45 \%$ regeneration of enzymatic activity when $\mathrm{CB}[7]$ was added to $\mathrm{BCA} \cdot \mathbf{I V}-\mathbf{1}$ due to more inhibition by 
$\mathrm{CB}[7] \cdot \mathbf{I V}-\mathbf{1}$ where as $83 \%$ regeneration was observed when $\mathrm{CB}[7]$ was added to BCA-IV-2. Finally Scheme IV-6 represents a complete shuttling of IV-2 between $\mathrm{BCA}$ and $\mathrm{CB}[7]$. A detail study of the mechanism of the shuttling process has been described above.

Scheme IV-6. A Complete Shuttling of IV-2 Between BCA and CB[7].

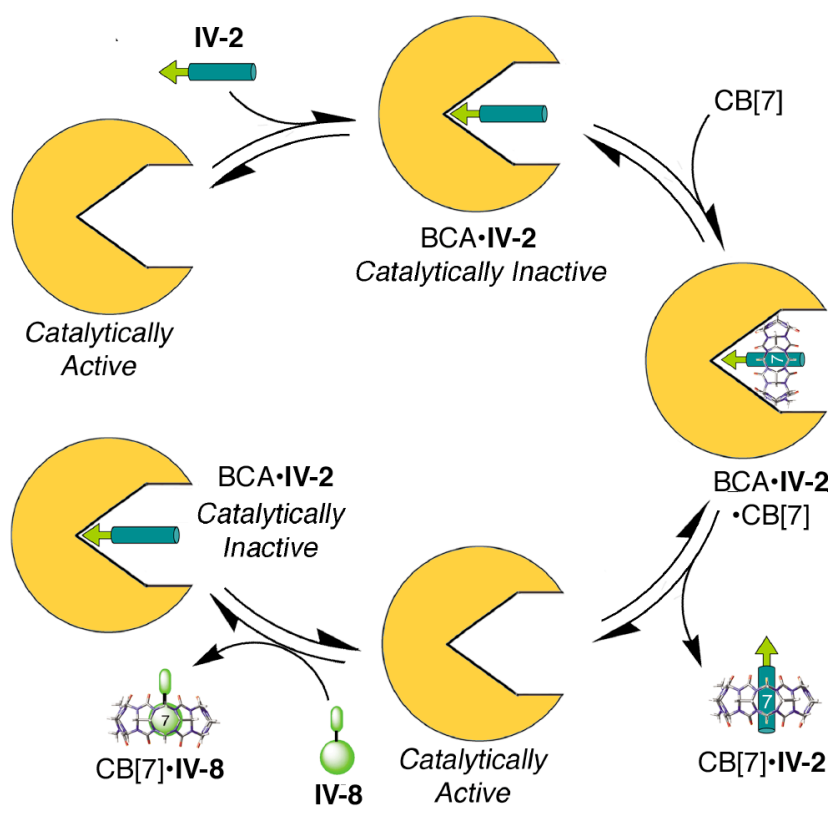

\subsubsection{Is the Shuttling of the Two-Faced Guest a Common Behavior?}

To address the question of whether all enzymes behave in a similar manner as BCA, we used compound IV-4 and IV-5 following the same design strategy where we linked a cationic amine group to the $\mathrm{AChE}$ inhibitor tacrine. Based on our experience with $\mathrm{BCA}$, we hypothesized a similar behavior under thermodynamic 
control where the addition of $\mathrm{CB}[7]$ would regenerate activity of $\mathrm{AChE}$ by sequestering IV-4 (or IV-5) from AChE•(IV-4) ${ }_{4}$ (or AChE•(IV-5) $)_{4}$.

\subsubsection{Regulation of Biological Catalysis of Acetylcholinesterase.}

When we measured the rate of hydrolysis of AChE by Ellman's assay before and after the addition of IV-4 or IV-5, we observed a decrease in rate of hydrolysis of IV-6 with a concomitant increase in concentration of IV-4 or IV-5. These results demonstrated that the tacrine subunits in IV-4 and IV-5 retained their inhibition capabilities even after derivatization. However, unlike BCA we did not observe any increase in the rate of hydrolysis of IV-6 after adding $\mathrm{CB}$ [7] to the solution of $\mathrm{AChE} \bullet(\mathbf{I V}-4)_{4}$ or $\mathrm{AChE} \bullet(\mathbf{I V}-5)_{4}$, a result that indicates no regeneration of enzymatic activity upon $\mathrm{CB}[7]$ addition. This observation was contrary to our hypothesis based on thermodynamics, since the $\mathrm{K}_{\mathrm{a}}$ values of tacrine derivatives to AChE that are known in the literature are much lower than $\mathrm{CB}$ [7]•adamantane ammonium ion interaction. $^{31}$

\subsubsection{Measurement of Association Constants.}

To assess the influence of thermodynamic parameters behind the experimental results, we determined the $\mathrm{K}_{\mathrm{a}}$ values for $\mathrm{AChE} \bullet$ inhibitor and $\mathrm{CB}[7] \bullet$ guest pairs. The

values $\mathrm{K}_{\mathrm{a}}$ of two-faced guests to $\mathrm{CB}[7]$ and $\mathrm{AChE}$ are summarized in Table IV-2. We measured the association constant of the inhibitor to AChE using Lineweaver- 
Burk analysis, which demonstrates a mixed inhibition behavior of IV-4 and IV-5 (Figure IV-6). ${ }^{174-176}$ A mixed inhibitor can bind to both enzyme and enzyme•inhibitor complex at a site distinct from the substrate active site. The association constant of IV-4 and IV-5 with CB[7] were measured by ${ }^{1} \mathrm{H}$ NMR competition experiments.
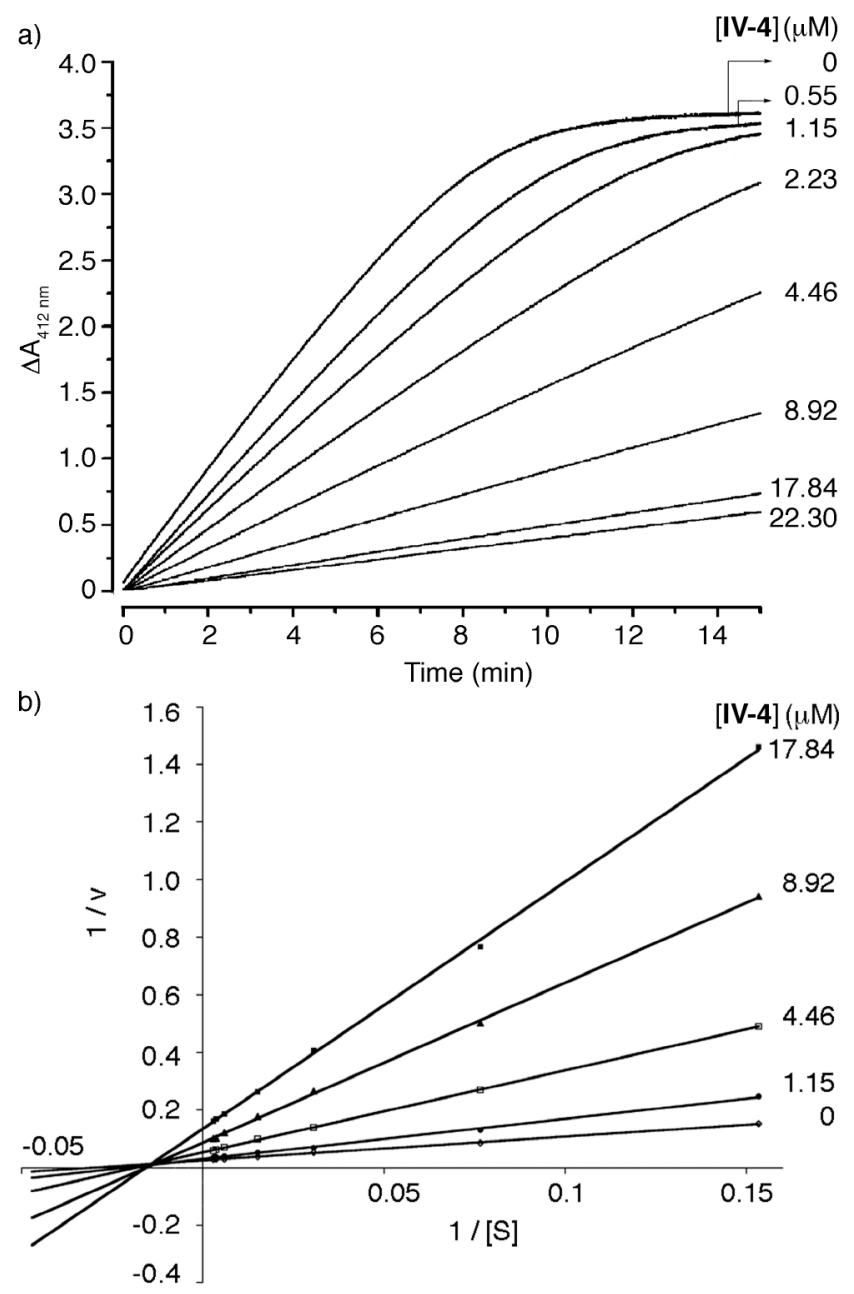

Figure IV-6. a) Titration of AChE with IV-4, and b) Lineweaver-Burk plot of AChE activity in the presence of $\mathbf{I V - 4}$. 
Table IV-2: Thermodynamic parameters for the interaction of IV-4 and IV-5 with $\mathrm{BCA}$ and $\mathrm{CB}[7]$.

\begin{tabular}{|c|c|c|}
\hline & $\mathrm{AChE}$ & $\mathrm{CB}[7]$ \\
\hline & $\mathrm{K}_{\mathrm{a}}\left(\mathrm{M}^{-1}\right)$ & $\mathrm{K}_{\mathrm{a}}\left(\mathrm{M}^{-1}\right)$ \\
\hline IV-4 & $\begin{array}{l}(1.92 \pm 0.02) \\
\times 10^{6 a} \\
(3.30 \pm 0.07) \\
\times 10^{6 a}\end{array}$ & $\begin{array}{l}(3.67 \pm 0.52) \\
\times 10^{12 b}\end{array}$ \\
\hline IV-5 & $\begin{array}{l}(4.19 \pm 0.50) \\
\times 10^{6 a} \\
(9.00 \pm 0.10) \\
\times 10^{5 a}\end{array}$ & $5.6 \times 10^{8 c}$ \\
\hline
\end{tabular}

${ }^{a}$ Measured by Lineweaver-Burk analysis.

${ }^{b}$ Measured by competition with IV-10 for a limiting quantity of CB[7].

${ }^{c}$ Measured by competition with IV-9 for a limiting quantity of CB[7].

Based on the values of $\mathrm{K}_{\mathrm{a}}$ and results of the colorimetric assays we considered two possibilities: First, $\mathrm{CB}[7]$ does not interact with $\mathrm{AChE} \bullet(\mathbf{I V}-4)_{4}$ or $\mathrm{AChE} \bullet(\mathbf{I V}-5)_{4}$ under the experimental conditions. Second, $\mathrm{CB}[7]$ binds to $\mathrm{AChE} \cdot(\mathbf{I V}-4)_{4}$ or $\mathrm{AChE} \bullet(\mathbf{I V}-5)_{4}$ to form a ternary complex $\mathrm{AChE} \bullet(\mathbf{I V}-\mathbf{4} \bullet \mathrm{CB}[7])_{4}$ or $\mathrm{AChE} \bullet(\mathbf{I V}$ $5 \cdot \mathrm{CB}[7])_{4}($ Scheme IV-7). In both cases the inhibitor occupies the active site of the enzyme in the presence of $\mathrm{CB}[7]$ and therefore the regeneration of the biological catalysis was not observed. Additionally, when we added carefully prepared 1:1 
mixture of $\mathrm{CB}[7] \cdot \mathbf{I V}-\mathbf{4}$ or $\mathrm{CB}[7] \cdot \mathbf{I V}-5$ to $\mathrm{AChE}$, we also observed a decrease in the rate of hydrolysis of IV-6. This result suggests that both $\mathrm{CB}[7] \cdot \mathbf{I V}-\mathbf{4}$ and $\mathrm{CB}[7] \cdot \mathbf{I V}-\mathbf{5}$ are capable of binding to the active site of AChE and therefore inhibit the catalytic activity.

\subsubsection{Fluorophore Displacement Assay.}

To ascertain the composition of the system after addition of $\mathrm{CB}$ [7] to $\mathrm{AChE} \bullet(\mathbf{I V}-4)_{4} \quad$ or $\mathrm{AChE} \bullet(\mathbf{I V}-4)_{4} \quad$ we used a fluorophore displacement assay. Compound IV-11 was used as the fluorogenic probe. ${ }^{177,178}$ Addition of CB[7] to IV11 results in the formation of CB[7] $/$ IV-11 with a concomitant blue shift of the emission wavelength from $509 \mathrm{~nm}$ to $485 \mathrm{~nm}$ and $32 \%$ increase in fluorescence intensity. When we added $\mathrm{AChE} \bullet(\mathbf{I V}-\mathbf{4})_{4}$ to a solution of $\mathrm{CB}[7] \bullet \mathbf{I V}-\mathbf{1 1}$ we observed a red shift from $485 \mathrm{~nm}$ to $509 \mathrm{~nm}$ (Figure IV-7). This result indicates $\mathrm{CB}$ [7] releasesIV-11 from its cavity and binds with $\mathrm{AChE} \bullet(\mathbf{I V}-\mathbf{4})_{4}$ to form the ternary complex AChE•(IV-4•CB[7] $)_{4}$. 


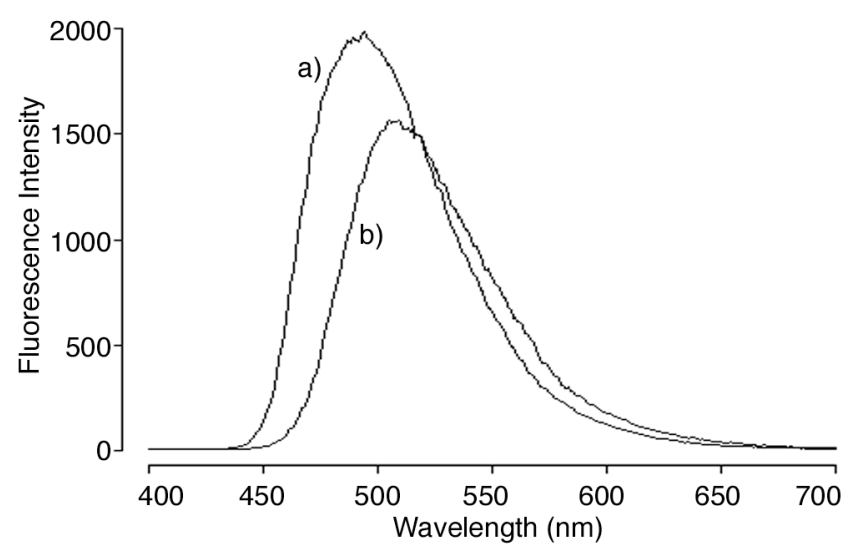

Figure IV-7. Fluorescence spectrum of a) a solution containing CB[7]•IV-11 and b) after addition of a solution containing $\mathrm{AChE} \bullet(\mathbf{I V}-\mathbf{4})_{4}$ to $\mathrm{CB}[7] \bullet \mathbf{I V}-\mathbf{1 1}$.

Scheme IV-7. Formation of Ternary Complex Upon Addition of CB[7] to $\mathrm{AChE} \bullet(\mathbf{I V}-4)_{4}$.

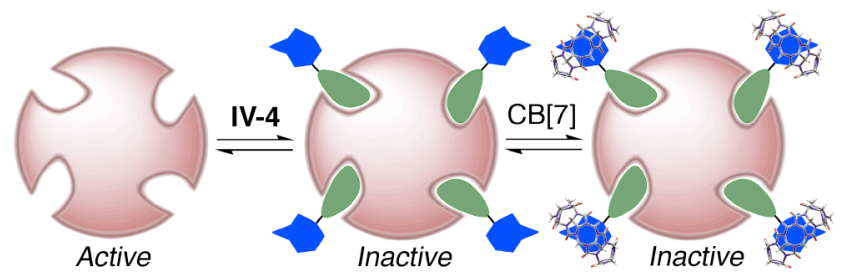

4.2.15 Effects of $\mathrm{CB}[7]$ in Controlling Enzymatic Activity of AChE.

While working with two-faced guests IV-4 and IV-5, we observed a weak inhibitory effect of $\mathrm{CB}[7]$ in the enzymatic activity of $\mathrm{AChE}$ in the control experiment. We observed a decrease in the enzymatic activity of AChE with a concomitant increase in concentration of $\mathrm{CB}[7]$ in Ellman's assay (Figure IV-8). 
Subsequently, we tested the binding of substrate IV-6 with CB[7] by ${ }^{1} \mathrm{H}-\mathrm{NMR}$. The upfield chemical shift of the quaternary ammonium group of IV-6 after addition of $\mathrm{CB}[7]$ indicates the formation of the $\mathrm{CB}[7] \cdot \mathbf{I V}-\mathbf{6}$ complex. The role of $\mathrm{CB}[7]$ was to enhances stability of the substrate IV-6 by reducing the rate of enzyme catalyzed degradation via host-guest complexation. ${ }^{179,180}$

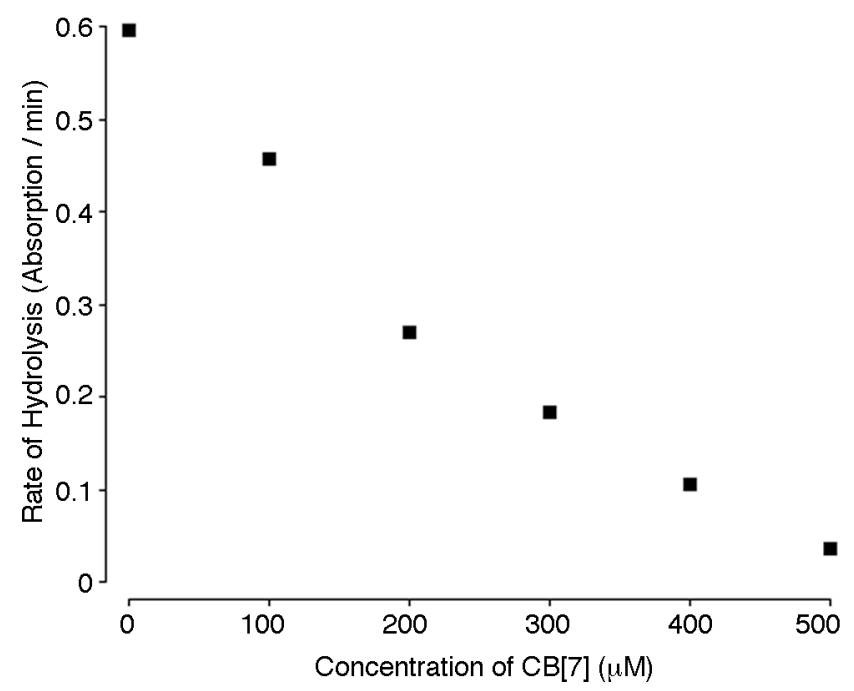

Figure IV-8. A plot of the rate of hydrolysis of IV-6 by AChE versus concentration of $\mathrm{CB}[7]$.

\subsubsection{The Origin of the Difference in the Behavior of Two Enzymes.}

We attribute the difference in behavior between two enzymes (Scheme IV-6 and IV-7) originates form the different nature of the active sites of these enzymes. Bovine carbonic anhydrase has $\sim 15 \AA$ deep and narrow conical binding site. Sulfonamide inhibitors bind to the $\mathrm{Zn}^{2+}$ cofactor, which is located at the bottom of the cleft. The interaction of the binding ligand with the $\mathrm{Zn}^{2+}$ cofactor is highly directional 
and majority of the $\mathrm{BCA} \cdot$ inhibitor interaction energy originated from this binding event. ${ }^{162}$ Therefore, the enzyme reacts sharply to any structural change in the inhibitor that leads to hindrance in binding (e.g. increase steric bulk, rigidity ${ }^{151}$ ). On the other hand Acetylcholinesterase is not a metalloprotease. Analysis of the crystal structure of AChE form Torpedo California (Due to unavailability of the crystal structure of AChE from Electrophorus Electricus, we used the crystal structure of AChE form Torpedo California for our analysis. AChE from both sources have substantial similarities in their properties and subunit composition ${ }^{181}$ ) shows that the active site of the enzyme is present in a $\sim 20 \AA \AA$ deep gorge which widens out near the base. At the rim of the gorge, $(\sim 14 \AA$ from the surface $) \mathrm{AChE}$ has an open peripheral binding site. ${ }^{182}$ The peripheral sites are flexible in nature and it can accommodate a wide variety of ligands of different shapes and sizes. Compounds IV-4 and IV-5 show mixed inhibition behavior, which indicates that they bind to peripheral site and reduce the rate of reaction by blocking IV-6 from accessing the active site. ${ }^{183}$ The inhibition capability of $\mathrm{CB}[7] \cdot \mathbf{I V}-\mathbf{4}$ and $\mathrm{CB}[7] \cdot \mathbf{I V}-5$ indicates that peripheral site has sufficient space for bulky host guest complexes to form a ternary complex. Formation of ternary protein $\bullet$ guest $\bullet \mathrm{CB}[\mathrm{n}]$ complexes is not uncommon in literature. Nau and coworkers reported formation of ternary complex bovine serum albumin•Brilliant Green $\bullet \mathrm{CB}[7] .{ }^{184}$ On the other hand, compounds IV-1 and IV-2 compete with the substrate for the active site of the enzyme. Compounds IV-1 and IV-2 fill up the active site cavity when they form the 1:1 complex with BCA. Upon complexation with $\mathrm{CB}[7]$ the steric bulk of the molecule increases by a substantial 
amount, which in turn disfavors the binding of $\mathrm{CB}[7] \bullet \mathbf{I V}-\mathbf{1}$ and $\mathrm{CB}[7] \bullet \mathbf{I V}-\mathbf{2}$ to the active site in the narrow cavity leading to a regeneration enzymatic activity.

\subsection{Conclusion.}

In the summary, we have described two-faced guest molecules that contain both enzyme inhibitor and cucurbit[n]uril binding domains. The activity of BCA can be regenerated by sequestering $\mathbf{I V - 1}$ and $\mathbf{I V - 2}$ from the active site of the enzyme using $\mathrm{CB}[7]$. Compound IV-2 was successfully used to turn-on and turn-off the enzymatic activity for eight steps. The incomplete regeneration of enzymatic activity upon addition of $\mathrm{CB}[7]$ to $\mathrm{BCA} \cdot \mathbf{I V}-\mathbf{1}$ and $\mathrm{BCA} \cdot \mathbf{I V - 2}$ due to inhibition by $\mathrm{CB}[7] \cdot \mathbf{I V}-\mathbf{1}$ and $\mathrm{CB}[7] \cdot \mathbf{I V}-\mathbf{2}$. Finally we demonstrated a limitation of this idea where addition of $\mathrm{CB}[7]$ to $\mathrm{AChE} \cdot \mathbf{I V}-\mathbf{4}_{4}$ and $\mathrm{AChE} \cdot \mathbf{I V}-\mathbf{5}_{4}$ results in the formation of a ternary complex that does not regenerate the enzymatic activity.

Apart from system observation described above, this study demonstrates a number of principles of broad applicability. Molecular recognition is fundamentally important in both biology and supramolecular chemistry, hence designed supramolecular systems can be useful in biology over time and space. Suitably designed synthetic modules can interact with their complementary target to control various phenomena such as folding, ion-transport through membrane, catalysis, replication, delivery have been demonstrated in the literature. The future direction of this research will involve the development of such systems in complex mixtures so that they are suitable for applications in vivo. Molecules containing multiple 
epitopes, each having some unique function can be very useful in this regard. Simply by picking examples from literature and connecting them by appropriate linker we can generate emergent properties in the molecules, which avoid time and difficulty of modeling and synthesizing entirely new molecule for achieving similar result. As our next challenge, we would like to move one step close to biology by replacing the nonnatural guest by another inhibitor and create similar systems using multiple enzymes for temporal control for the activity of the biological receptors.

\subsection{Experimental.}

\subsubsection{General Experimental.}

Starting materials were purchased from Alfa-Aesar, Acros, and Aldrich and were used without further purification. Bovine Carbonic Anhydrase (Sigma C3934), Acetylcholinesterase (Sigma C3389), compounds IV-6, IV-7, and IV-9 - IV-13 were obtained from commercial sources. Melting points were measured on a Meltemp apparatus in open capillary tubes and are uncorrected. NMR spectra were measured

on Bruker AM-400 and DRX-400 at $400 \mathrm{MHz}$ for ${ }^{1} \mathrm{H}$ and $100 \mathrm{MHz}$ for ${ }^{13} \mathrm{C}$. Mass spectrometry was performed using a VG 7070E magnetic sector instrument by electron impact (EI) or by fast atom bombardment (FAB) using the indicated matrix. The matrix "magic bullet" is a 5:1 (w:w) mixture of dithiothreitol:dithioerythritol. 


\subsubsection{Synthetic Procedures and Characterization.}

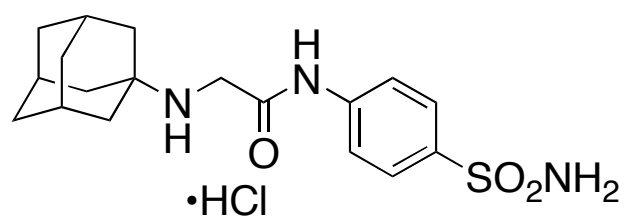

Compound IV-1: Compound IV-16 (838 mg, $3.36 \mathrm{mmol})$ and adamantane amine $(510 \mathrm{mg}$, $3.36 \mathrm{mmol})$ were dissolved in anh. THF (50 $\mathrm{mL}$ ) under $\mathrm{N}_{2}$. To that mixture, $\mathrm{K}_{2} \mathrm{CO}_{3}(1.39 \mathrm{~g}, 10.08 \mathrm{mmol})$ and $\mathrm{KI}(390 \mathrm{mg}, 2.35$ mmol) were added and reflux was continued for $15 \mathrm{~h}$. The reaction mixture was concentrated using rotary evaporator and was washed was washed with distilled water $(2 \times 5 \mathrm{~mL})$, centrifuged, the supernatant decanted and the residue dried at high vacuum to get impure amine $\mathbf{I V - 1}$. The impure solid was washed with $\mathrm{CHCl}_{3}(1$ $\mathrm{mL}$ ), centrifuged, the supernatant decanted and the residue dried at high vacuum to obtain pure amine IV-1 as a white solid (887 mg, $2.44 \mathrm{mmol}, 73 \%)$. Hydrochloric acid salt of IV-1 was prepared by adding $\mathrm{HCl}$ gas to a solution of pure amine $\mathbf{I V}-\mathbf{1}$ in EtOH. M.p. $>310{ }^{\circ} \mathrm{C} . \quad$ IR $\left(\mathrm{KBr}, \mathrm{cm}^{-1}\right): 3250 \mathrm{w}, 2918 \mathrm{~m}, 2950 \mathrm{w}, 1702 \mathrm{~s}, 1599 \mathrm{~m}$, 1539s, 1402m, 1323s, 1166s. ${ }^{1} \mathrm{H}$ NMR (400 MHz, DMSO-d $)$ ): 11.10 (s, 1H), 8.94 (s, 2H), $7.82(\mathrm{~d}, J=8.9,2 \mathrm{H}), 7.78(\mathrm{~d}, J=8.9,2 \mathrm{H}), 7.32(\mathrm{~s}, 2 \mathrm{H}), 3.98(\mathrm{~s}, 2 \mathrm{H}), 2.14(\mathrm{~s}$, $3 \mathrm{H}), 1.87(\mathrm{~s}, 6 \mathrm{H}), 1.68(\mathrm{~d}, J=12.2,3 \mathrm{H}), 1.58(\mathrm{~d}, J=12.2,3 \mathrm{H}) .{ }^{13} \mathrm{C} \mathrm{NMR}(100 \mathrm{MHz}$, $\left.\mathrm{CDCl}_{3}\right): \delta 165.0,141.1,139.1,126.9,118.8,56.6,41.4,37.5,35.2,28.4$. MS (FAB, glycerol/DMSO): $m / z 364\left(4.5,[\mathrm{M}-\mathrm{Cl}]^{+}\right), 89$ (100). HR-MS (FAB, PEG): $m / z$ $364.1695\left([\mathrm{M}-\mathrm{Cl}]^{+}, \mathrm{C}_{18} \mathrm{H}_{26} \mathrm{~N}_{3} \mathrm{O}_{3} \mathrm{~S}\right.$, calcd 364.1688).

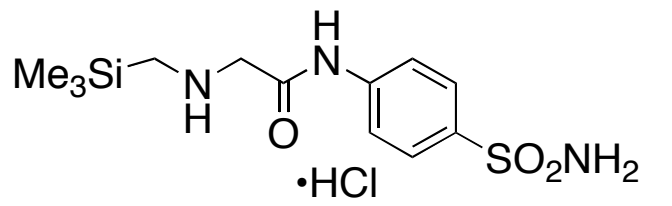

Compound IV-2: Compound IV-16 (600 mg, $2.41 \mathrm{mmol})$ was dissolved in $\mathrm{CH}_{3} \mathrm{CN}(30 \mathrm{~mL})$ 
under $\mathrm{N}_{2}$ and compound (trimethylsilyl)methyl amine (248 $\mathrm{mg}, 2.41 \mathrm{mmol}$ ) was added in one portion. To that mixture, $\mathrm{K}_{2} \mathrm{CO}_{3}(997 \mathrm{mg}, 7.22 \mathrm{mmol})$ and $\mathrm{KI}(280 \mathrm{mg}$, $1.68 \mathrm{mmol}$ ) were added and reflux was continued for $15 \mathrm{~h}$. The reaction mixture was concentrated using rotary evaporator and was washed was washed with acetone $(2 \times$ $30 \mathrm{~mL}$ ), centrifuged, the supernatant was concentrated to get impure amine IV-2. Flash chromatography $\left(\mathrm{SiO}_{2}, \mathrm{CHCl}_{3} / \mathrm{MeOH} / \mathrm{NH}_{4} \mathrm{OH}\right.$ 100:5:1) gave pure amine IV-2 as a white solid (470 mg, $1.49 \mathrm{mmol}, 62 \%$ ). Hydrochloric acid salt of IV-2 was prepared by adding $\mathrm{HCl}$ gas to a solution of pure amine IV-2 in EtOH. M.p. 224-227 ${ }^{\circ} \mathrm{C}$. IR (KBr, cm $\left.{ }^{-1}\right): 3380 \mathrm{w}, 2962 \mathrm{w}, 1697 \mathrm{~m}, 1544 \mathrm{~m}, 1322 \mathrm{~m}, 1156 \mathrm{~s} .{ }^{1} \mathrm{H}$ NMR (400 $\left.\mathrm{MHz}, \mathrm{D}_{2} \mathrm{O}\right): 7.93(\mathrm{~d}, J=8.8,2 \mathrm{H}), 7.72(\mathrm{~d}, J=8.8,2 \mathrm{H}), 4.08(\mathrm{~s}, 2 \mathrm{H}), 2.65(\mathrm{~s}, 2 \mathrm{H})$, 0.22 (s, 9H). ${ }^{13} \mathrm{C}$ NMR $\left(100 \mathrm{MHz}, \mathrm{CDCl}_{3}\right): \delta 164.5,141.1,139.0,126.8,118.9,51.8$, 37.3, -2.0. MS (FAB, glycerol/DMSO): $m / z 316\left(100,[\mathrm{M}-\mathrm{Cl}]^{+}\right)$. HR-MS (FAB, PEG): $m / z 316.1151\left([\mathrm{M}-\mathrm{Cl}]^{+}, \mathrm{C}_{12} \mathrm{H}_{22} \mathrm{~N}_{3} \mathrm{O}_{3} \mathrm{SSi}\right.$, calcd 316.1158).

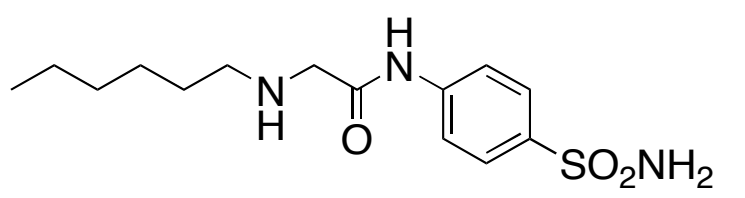

Compound IV-3: Compound IV-16 (388 $\mathrm{mg}, 1.56 \mathrm{mmol})$ and hexylamine (237 mg, $2.34 \mathrm{mmol})$ were dissolved in anh. THF $(15 \mathrm{~mL})$ under $\mathrm{N}_{2}$. To that mixture, $\mathrm{K}_{2} \mathrm{CO}_{3}(646 \mathrm{mg}, 4.68 \mathrm{mmol})$ and $\mathrm{KI}(181 \mathrm{mg}, 1.09 \mathrm{mmol})$ were added and reflux was continued for $17 \mathrm{~h}$. The reaction mixture was concentrated using rotary evaporator and was washed was washed with acetone $(2 \times 20 \mathrm{~mL})$, centrifuged, the supernatant was concentrated to get impure amine IV-3. Flash chromatography $\left(\mathrm{SiO}_{2}\right.$, $\mathrm{CHCl}_{3} / \mathrm{MeOH} / \mathrm{NH}_{4} \mathrm{OH}$ 100:5:1) gave pure amine IV-3 as a white solid (333 mg, 1.06 mmol, $68 \%)$. M.p. $172-175{ }^{\circ} \mathrm{C} . \quad \mathrm{TLC}\left(\mathrm{CHCl}_{3} / \mathrm{MeOH} 1: 1\right) R_{\mathrm{f}} 0.2$. IR $\left(\mathrm{KBr}, \mathrm{cm}^{-1}\right)$ : 
3257w, 2927w, 1687m, 1594m, 1514m, 1323m, 1151s. ${ }^{1} \mathrm{H}$ NMR (400 MHz, DMSO$d_{6}$ ): 10.30 (br. s, 1H), 7.79 (d, $\left.J=9.0,2 \mathrm{H}\right), 7.75(\mathrm{~d}, J=9.0,2 \mathrm{H}), 7.25(\mathrm{~s}, 2 \mathrm{H}), 3.30$ (s, $2 \mathrm{H}), 2.52(\mathrm{t}, J=7.1,2 \mathrm{H}), 1.45-1.40(\mathrm{~m}, 2 \mathrm{H}), 1.35-1.25(\mathrm{~m}, 6 \mathrm{H}), 0.86(\mathrm{t}, J=7.1,3 \mathrm{H})$. ${ }^{13} \mathrm{C}$ NMR $\left(100 \mathrm{MHz}, \mathrm{CDCl}_{3}\right): \delta 171.1,141.6,138.4,126.7,118.7,52.9,49.1,31.2$, 29.4, 26.4, 22.1, 14.0. MS (FAB, glycerol/DMSO): $m / z 314\left(100,[\mathrm{M}+\mathrm{H}]^{+}\right)$. HRMS (FAB, magic bullet, PEG): $m / z 314.1538\left([\mathrm{M}+\mathrm{H}]^{+}, \mathrm{C}_{14} \mathrm{H}_{24} \mathrm{~N}_{3} \mathrm{O}_{3} \mathrm{~S}\right.$, calcd $314.1531)$.

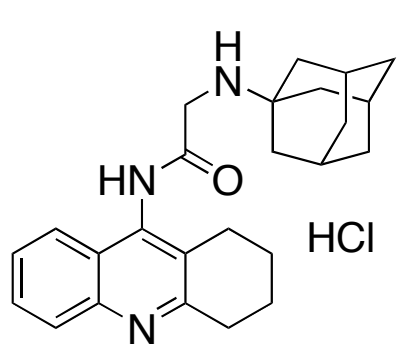

Compound IV-4: Compound IV-18 (107 mg, 0.39 mmol) and adamantane amine $(107 \mathrm{mg}, 0.7 \mathrm{mmol})$ were dissolved in $\mathrm{CH}_{3} \mathrm{CN}(8 \mathrm{~mL})$ under $\mathrm{N}_{2}$. To that mixture, $\mathrm{K}_{2} \mathrm{CO}_{3}(164$ $\mathrm{mg}, 4.68 \mathrm{mmol})$ and $\mathrm{KI}(47 \mathrm{mg}, 1.09 \mathrm{mmol})$ were added and reflux was continued for $14 \mathrm{~h}$. The reaction mixture was concentrated using rotary evaporator and was washed was washed with acetone $(2 \times 10 \mathrm{~mL})$, centrifuged, the supernatant was concentrated to get impure residue. Pure amine IV-4 was obtained after recrystallization from $\mathrm{EtOH}$ as a white solid (92 $\mathrm{mg}, 0.24 \mathrm{mmol}, 62 \%)$. Hydrochloric acid salt of IV-4 was prepared by adding $\mathrm{HCl}$ gas to a solution of pure amine IV-4 in EtOH. M.p. $>250{ }^{\circ} \mathrm{C}$ (dec.). IR (KBr, $\left.\mathrm{cm}^{-1}\right)$ : $3412 \mathrm{w}, 2911 \mathrm{~m}, 2681 \mathrm{~s}$, 1703s, 1641m, 1464m, 1388s. ${ }^{1} \mathrm{H}$ NMR (400 MHz, $\left.\mathrm{D}_{2} \mathrm{O}\right): 8.11(\mathrm{~d}, J=8.8,2 \mathrm{H}), 8.05$ (t, $J=7.8,1 \mathrm{H}), 7.87(\mathrm{t}, J=7.8,1 \mathrm{H}), 4.40(\mathrm{~s}, 2 \mathrm{H}), 3.36(\mathrm{t}, J=6.3,2 \mathrm{H}), 2.89(\mathrm{t}, J=$ 6.3, 2H), 2.25 (s, 3H), 2.05-1.85 (m, 10H), 1.79 (d, $J=12.5,3 \mathrm{H}), 1.70$ (d, $J=12.5$, 3H). ${ }^{13} \mathrm{C}$ NMR (100 MHz, $\left.\mathrm{CDCl}_{3}\right): \delta 165.4,158.3,133.0,128.3,127.9,125.4,123.4$, 
$56.7,41.2,37.6,35.2,28.5,28.3,24.8,20.8,20.6$ (only 16 of the 19 expected resonances were observed).

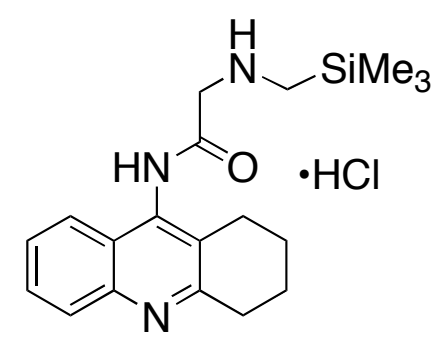

Compound IV-5: Compound IV-18 (50 mg, $0.18 \mathrm{mmol})$ was dissolved in $\mathrm{CH}_{3} \mathrm{CN}(8 \mathrm{~mL})$ under $\mathrm{N}_{2}$ and compound (trimethylsilyl)methyl amine (62 $\mathrm{mg}, 0.59 \mathrm{mmol})$ was added in one portion. To that mixture, $\mathrm{K}_{2} \mathrm{CO}_{3}(75 \mathrm{mg}, 0.54$ $\mathrm{mmol})$ and $\mathrm{KI}(21 \mathrm{mg}, 0.12 \mathrm{mmol})$ were added and reflux was continued for $18 \mathrm{~h}$. The reaction mixture was concentrated using rotary evaporator and was washed was washed with acetone $(2 \times 15 \mathrm{~mL})$, centrifuged, the supernatant was concentrated to get impure IV-5. Flash chromatography $\left(\mathrm{SiO}_{2}, \mathrm{CHCl}_{3} / \mathrm{MeOH} / \mathrm{NH}_{4} \mathrm{OH}\right.$ 100:5:1) gave pure IV-5 as a white solid (40 mg, $0.12 \mathrm{mmol}, 67 \%)$. M.p. $128-130^{\circ} \mathrm{C}$. IR (KBr, $\mathrm{cm}^{-}$ $\left.{ }^{1}\right)$ : 3341w, 2935w, 2730w, 2605w, 1703m, 1583m, 1512m, 1250s. ${ }^{1} \mathrm{H}$ NMR (400 $\left.\mathrm{MHz}, \mathrm{D}_{2} \mathrm{O}\right): 8.11(\mathrm{t}, J=7.4,2 \mathrm{H}), 8.05(\mathrm{t}, J=7.4,1 \mathrm{H}), 7.87(\mathrm{t}, J=7.4,1 \mathrm{H}), 4.40(\mathrm{~s}$, $2 \mathrm{H}), 3.36(\mathrm{t}, J=6.3,2 \mathrm{H}), 2.90(\mathrm{t}, J=6.3,2 \mathrm{H}), 2.71(\mathrm{~s}, 2 \mathrm{H}), 2.05-2.00(\mathrm{~m}, 2 \mathrm{H}), 1.95-$ $1.90(\mathrm{~m}, 2 \mathrm{H}),-0.22(\mathrm{~s}, 9 \mathrm{H}) .{ }^{13} \mathrm{C}$ NMR $\left(100 \mathrm{MHz}, \mathrm{DMSO}-d_{6}\right): \delta 164.9,158.1,146.8$, $137.3,133.3,128.4,128.2,125.5,123.4,120.1,51.7,37.4,28.8,24.9,20.7,20.4$, 1.9. 


\subsection{3 ${ }^{1} \mathrm{H}$ NMR Competition Experiments.}

${ }^{1} \mathrm{H}$ NMR competition experiments were performed on a $400 \mathrm{MHz} \mathrm{NMR}$ spectrometer. The temperature was maintained at $298 \pm 0.5 \mathrm{~K}$ with a temperature control module that had been calibrated using the separation of the resonances of methanol. Each sample contained CB[7] and an excess of the two competitive guests. ${ }^{1} \mathrm{H}$ NMR spectra were acquired with a delay time $10 \mathrm{~s}$. All spectra were referenced to $\mathrm{D}_{2} \mathrm{O}$ at $4.79 \mathrm{ppm}$.

\section{Equations:}

$$
\begin{aligned}
& \mathrm{CB}[\mathrm{n}]+\mathrm{G} 1 \stackrel{\mathrm{K}_{\mathrm{G} 1}}{\rightleftharpoons} \mathrm{CB}[\mathrm{n}] \cdot \mathrm{G} 1 \\
& \mathrm{CB}[\mathrm{n}]+\mathrm{G} 2 \stackrel{\mathrm{K}_{\mathrm{G} 2}}{\rightleftharpoons} \mathrm{CB}[\mathrm{n}] \cdot \mathrm{G} 2 \\
& \mathrm{CB}[\mathrm{n}] \cdot \mathrm{G} 1+\mathrm{G} 2 \stackrel{\mathrm{K}_{\mathrm{rel}}}{\rightleftharpoons} \mathrm{CB}[\mathrm{n}] \cdot \mathrm{G} 2+\mathrm{G} 1 \\
& K_{\mathrm{rel}}=([\mathrm{CB}[\mathrm{n}] \cdot \mathrm{G} 2][\mathrm{G} 1]) /([\mathrm{CB}[\mathrm{n}] \cdot \mathrm{G} 1][\mathrm{G} 2]) \\
& K_{\mathrm{G} 2}=\left(K_{\mathrm{G} 1}\right)\left(K_{\mathrm{rel}}\right)
\end{aligned}
$$


4.4.3.1 Sample Determination of $\mathrm{K}_{\mathrm{a}}$ of two-faced guest with $\mathrm{CB}[7]$ :
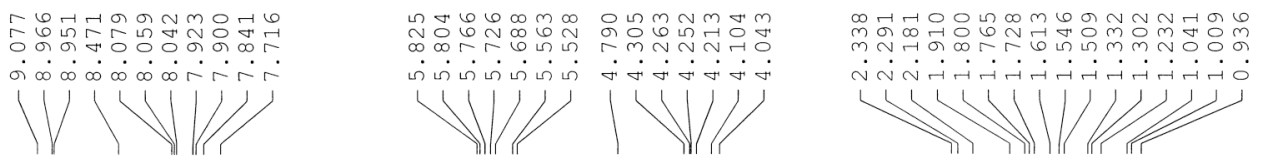

Determination of the $\mathrm{K}_{\mathrm{a}}$ of:

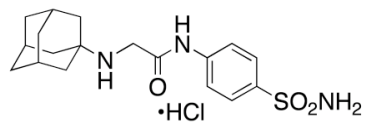

$400 \mathrm{MHz}, \mathrm{D}_{2} \mathrm{O}$

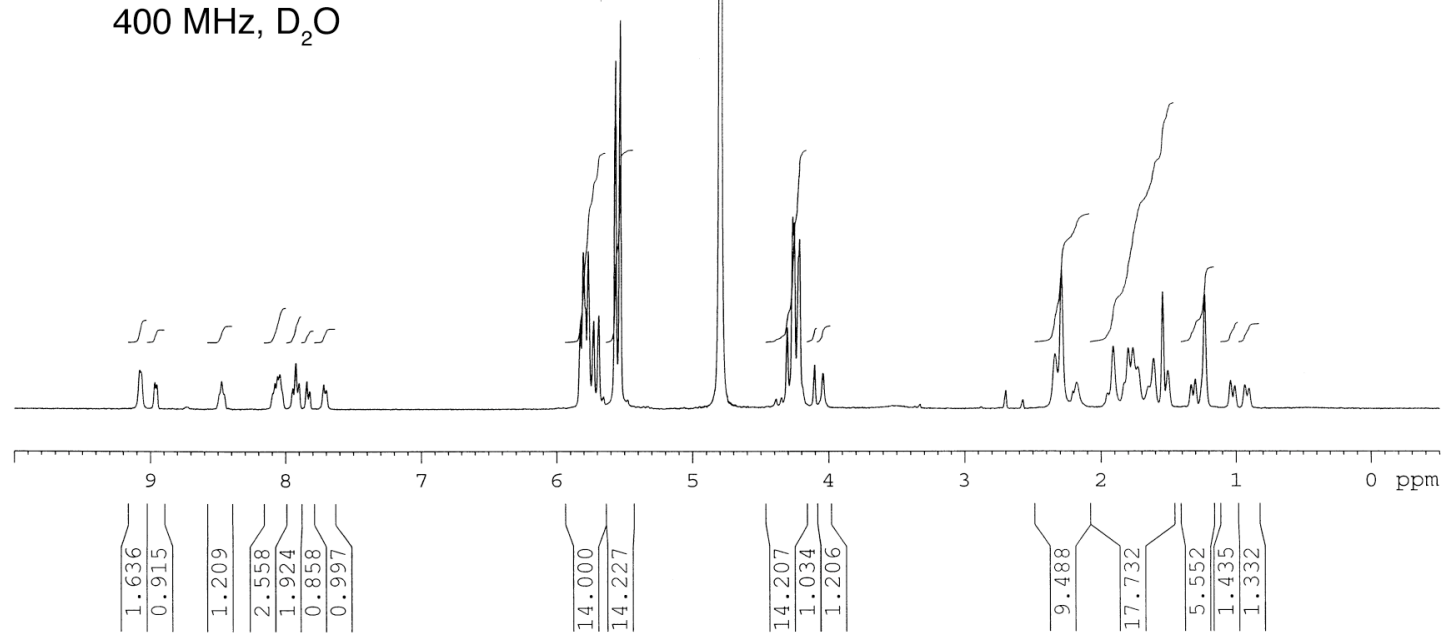

Figure IV-9: Sample Determination of the binding constant of IV-1

Sequence of addition: Addition of IV-1 followed by the addition of IV-10:

$[\mathrm{CB}[7]]_{\text {Total }}=1.44 \mathrm{mM} ;[\mathbf{I V - 1}]_{\text {Total }}=2.01 \mathrm{mM} ;[\mathbf{I V}-\mathbf{1 0}]_{\text {Total }}=2.01 \mathrm{mM}$

Sample Determination of $\mathrm{K}_{\text {rel }}$ :

From ${ }^{1} \mathrm{H}-\mathrm{NMR}$ we determined $[\mathbf{I V}-\mathbf{1}]_{\text {Free }}=1.08 \mathrm{mM}$ and $[\mathrm{CB}[7] \cdot \mathbf{I V}-\mathbf{1}]=0.93 \mathrm{mM}$ $[\mathrm{CB}[7] \cdot \mathbf{I V}-\mathbf{1 0}]=[\mathrm{CB}[7]]_{\mathrm{Total}}-[\mathrm{CB}[7] \cdot \mathbf{I V}-\mathbf{1}]=0.51 \mathrm{mM}$

$[\mathbf{I V}-\mathbf{1 0}]_{\text {Free }}=[\mathbf{I V}-\mathbf{- 1 0}]_{\text {Total }}-[\mathrm{CB}[7] \cdot \mathbf{I V}-\mathbf{1 0}]=1.50 \mathrm{mM}$

$\mathrm{K}_{\mathrm{rel}}=(0.51 \times 1.08) /(0.93 \times 1.5)=0.39$

$\mathrm{K}_{\mathrm{a}}=1.98 \times 10^{8} / 0.39 \mathrm{M}^{-1}=5.07 \times 10^{12} \mathrm{M}^{-1}$ 
Addition of IV-10 followed by the addition of IV-1.

$[\mathrm{CB}[7]]_{\text {Total }}=1.44 \mathrm{mM} ;[\mathbf{I V}-\mathbf{1 0}]_{\text {Total }}=2.01 \mathrm{mM} ;[\mathbf{I V}-\mathbf{1}]_{\text {Total }}=2.41 \mathrm{mM}$

From ${ }^{1} \mathrm{H}-\mathrm{NMR}$ we determined $[\mathbf{I V}-\mathbf{1}]_{\text {Free }}=1.51 \mathrm{mM}$ and $[\mathrm{CB}[7] \cdot \mathbf{I V}-\mathbf{1}]=0.90 \mathrm{mM}$

$\mathrm{K}_{\text {rel }}=1.62 ; \mathrm{K}_{\mathrm{a}}=3.20 \times 10^{12} \mathrm{M}^{-1} ;$ Mean $\mathrm{K}_{\mathrm{a}}=4.13 \times 10^{12} \mathrm{M}^{-1}$

\subsubsection{UV/Vis Experiments.}

UV/Vis spectra were recorded on a Cary 100-Bio UV-Visible spectrophotometer using $1 \mathrm{~cm}$ pathlength cells. The temperature was held constant at $25{ }^{\circ} \mathrm{C}$ using RTE bath / circulator containing a microprocessor controller. The kinetic assays were performed using literature procedures in the stated buffer. ${ }^{164-166}$ The relative rates of reaction were determined from initial slope of plots of $\Delta \mathrm{A}$ vs time.

4.4.4.1 Determination of Enzymatic Activity of Acetylcholinesterase.

a) Preparation of Solutions:

Substrate: $113 \mathrm{mg}$ of IV-6 was dissolve in $5 \mathrm{~mL}$ K-Phosphate buffer (Concentration: $78.15 \mathrm{mM})$. The stock solution was stored at $4{ }^{\circ} \mathrm{C}$.

Reagent: $42 \mathrm{mg}$ of IV-13 was dissolved in $10 \mathrm{~mL}$ K-Phosphate buffer (pH 7.0) and $17 \mathrm{mg}$ of $\mathrm{NaHCO}_{3}$ was added to that solution (Concentration: $10.59 \mathrm{mM}$ ). 
Enzyme: $1.17 \mathrm{mg}$ enzyme was purchased as Lyophilized powder containing Tris buffer salts and was dissolved in $1 \mathrm{~mL} \mathrm{K-Phosphate} \mathrm{buffer.} \mathrm{The} \mathrm{stock} \mathrm{solution} \mathrm{was}$ stored at $-20{ }^{\circ} \mathrm{C}$. The final solution used in the assay contained 5.35 unit protein $/ \mathrm{mL}$. By definition one unit hydrolyzes $1.0 \mu$ mole of acetylcholine to choline and acetate per min at $\mathrm{pH} 8.0$ at $37^{\circ} \mathrm{C}$.

Buffer: $20 \mathrm{mM}$ K-Phosphate Buffer, pH 8.0; Temperature: $25^{\circ} \mathrm{C}$

b) Determination of Michalis Constant for Acetylcholinesterase.

The substrate (IV-6) concentration was gradually increased keeping the AChE concentration fixed until the reaction velocity reached its maximum saturation value. The concentration of substrate was varied from $6.51 \mu \mathrm{M}$ to $325 \mu \mathrm{M}$. The $\mathrm{K}_{\mathrm{M}}$ value was measured by fitting Equation IV.8 to a plot of rate of enzyme hydrolysis versus concentration of substrate. The final solution used in the assay contained 5.35 unit protein $/ \mathrm{mL}$.

The blank was measured with $50 \mu \mathrm{L}$ Enzyme, $100 \mu \mathrm{L}$ IV-13 and $2850 \mu \mathrm{L}$ buffer. In a typical run $50 \mu \mathrm{L}$ Enzyme, $100 \mu \mathrm{L}$ IV-13 and $2840 \mu \mathrm{L}$ buffer were incubated at $25{ }^{\circ} \mathrm{C}$ for 15 mins. To that solution $10 \mu \mathrm{L}$ IV-6 was added and the change of absorbance was monitored at $412 \mathrm{~nm}$ using by UV/Vis spectroscopy. The rate of the reaction was measured from the slope of the initial linear portion of the curve. 


$$
\begin{aligned}
& \mathrm{E}+\mathrm{S} \underset{\mathrm{k}_{-1}}{\stackrel{\mathrm{k}_{1}}{\longrightarrow}} \mathrm{ES} \stackrel{\mathrm{k}_{\text {cat }}}{\longrightarrow} \mathrm{EP} \longrightarrow \mathrm{E}+\mathrm{P} \\
& \mathrm{v}=\left(\mathrm{v}_{\max }[\mathrm{S}]\right) /\left(\mathrm{K}_{\mathrm{M}}+[\mathrm{S}]\right)
\end{aligned}
$$

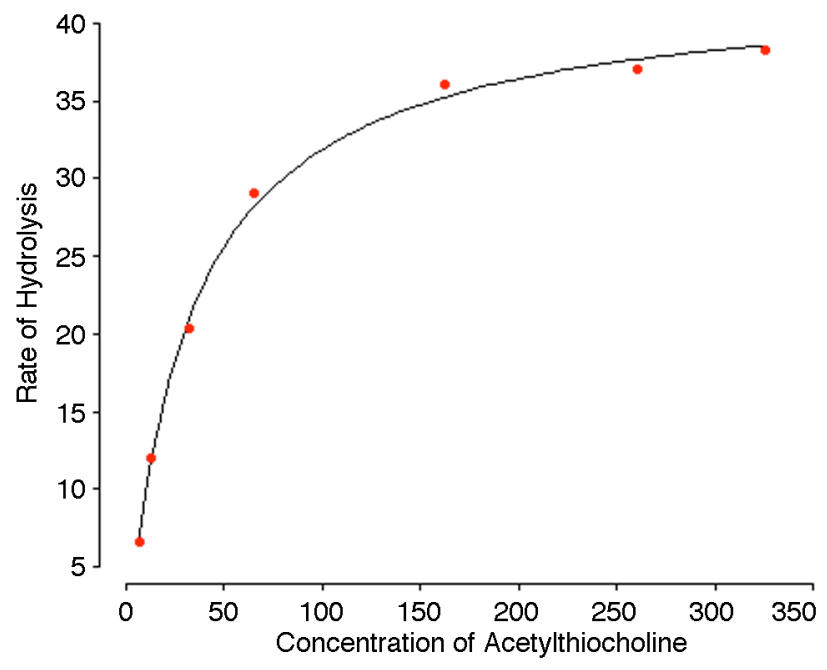

Figure IV-10. Plot of rate of hydrolysis versus concentration of acetylthiocholine.

The value of $\mathrm{K}_{\mathrm{M}}$ and $\mathrm{V}_{\mathrm{Max}}$ calculated from Figure IV-10 are $(33.21 \pm 1.93) \mu \mathrm{M}$ and $(42.52 \pm 0.64) \mu \mathrm{M} \mathrm{m^{-1 }} \mathrm{g}^{-1}$, respectively.

c) A Sample Determination of Dissociation Constant of IV-4 with AChE.

Preparation Solutions:

Preparation of a solution of IV-4: $0.0057 \mathrm{mg} \mathbf{I V}-\mathbf{4}$ was dissolved in $10 \mathrm{~mL}$ deionized water (Concentration $1.34 \mathrm{mM}$ ). 
Preparation of a solution of IV-5: $0.0038 \mathrm{mg}$ IV-5 was dissolved in $10 \mathrm{~mL}$ deionized water (Concentration $1.01 \mathrm{mM})$.

Same stock solutions for IV-6, IV-13 and enzymes reported previously were used.

Buffer: 20 mM K-Phosphate Buffer, $\mathrm{pH} 8.0$; Temperature: $25^{\circ} \mathrm{C}$

Two sets of experiments were carried out to determine the nature of inhibition with the enzyme concentration held constant in each set. In the first set, inhibitor held constant and the effect of the concentration substrate $(6.51-325 \mu \mathrm{M})$ on the initial rate of hydrolysis was determined. In the next set of experiments, the effect of variable inhibitor concentration $(1.15 \mu \mathrm{M}, 4.46 \mu \mathrm{M}, 8.92 \mu \mathrm{M}$, and $17.84 \mu \mathrm{M})$ was measured over the fixed range of concentration of substrate $(6.51-325 \mu \mathrm{M})$.

The blank was measured with $50 \mu \mathrm{L}$ Enzyme, $100 \mu \mathrm{L}$ IV-13 and $2850 \mu \mathrm{L}$ buffer. In a typical run, enzyme (50 $\mu \mathrm{L}), \mathbf{I V}-\mathbf{1 3}(100 \mu \mathrm{L})$ in buffer, inhibitor IV-4 (10 $\mu \mathrm{L}$ ) was added in $2830 \mu \mathrm{L}$ buffer and the solution was incubated at $25{ }^{\circ} \mathrm{C}$ for 15 mins. To that mixture $10 \mu \mathrm{L}$ IV-6 was added and the rate of the reaction was monitored by measuring the change of absorbance at $412 \mathrm{~nm}$ using by UV/Vis spectroscopy. The dissociation constant of the inhibitor was measured using Lineweaver-Burk analysis (Equation IV.9 - IV.11).

\section{Equation Representing a Mixed Inhibition:}

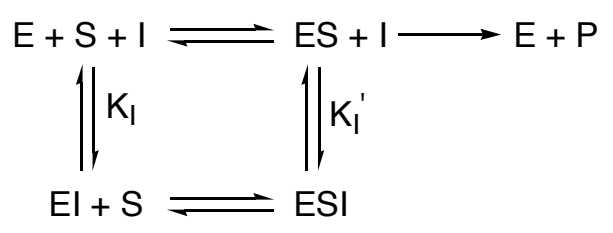


$\mathrm{v}=\left(\mathrm{v}_{\max }[\mathrm{S}]\right) /\left(\alpha \mathrm{K}_{\mathrm{M}}+\alpha^{\prime}[\mathrm{S}]\right)$

$\left(1 / \mathrm{V}_{0}\right)=\left(\alpha \mathrm{K}_{\mathrm{m}} / \mathrm{V}_{\max }\right)(1 /[\mathrm{S}])+\left(\alpha^{\prime} / \mathrm{V}_{\max }\right)$

where, $\alpha=1+\left([\mathrm{I}] / \mathrm{K}_{\mathrm{I}}\right)$

and $\left.\quad \alpha^{\prime}=1+\left([\mathrm{I}] / \mathrm{K}_{\mathrm{I}}\right)^{\prime}\right)$

The values of $\alpha$ and $\alpha$ ' were calculated from the known values of $\mathrm{K}_{\mathrm{m}}$ and $\mathrm{V}_{\max } \mathrm{using}$ equation 4.7. The values of $\mathrm{K}_{\mathrm{I}}$ and $\mathrm{K}_{\mathrm{I}}$ ' were calculated form the reciprocal of the slopes when $\alpha$ and $\alpha$ ' were plotted with respect to [I] (Figure IV-11a and IV-11b).

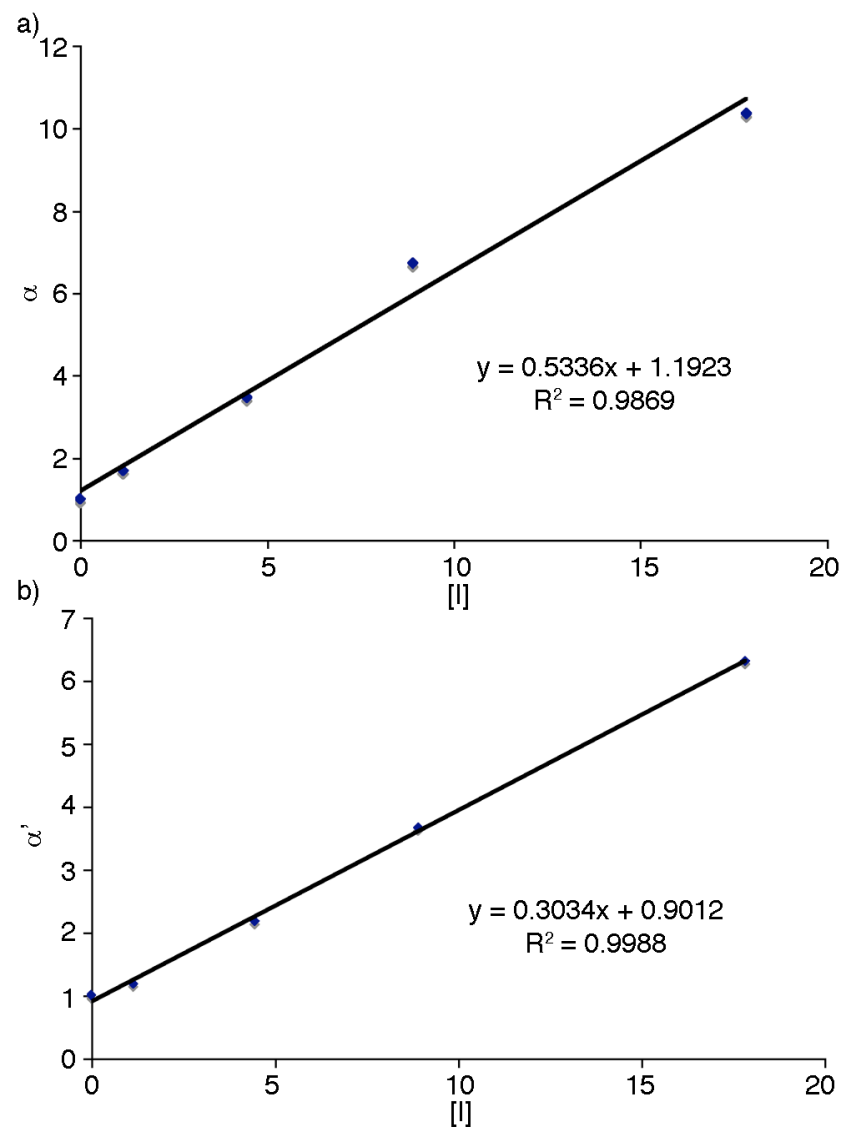

Figure IV-11. a) Plot of $\alpha$ versus [IV-4]; and b) Plot of $\alpha^{\prime}$ versus [IV-4]. 
The values of $\alpha$ and $\alpha^{\prime}$ calculated from Figure IV-11 are $1.87 \mu \mathrm{M}$ and $3.29 \mu \mathrm{M}$ respectively.

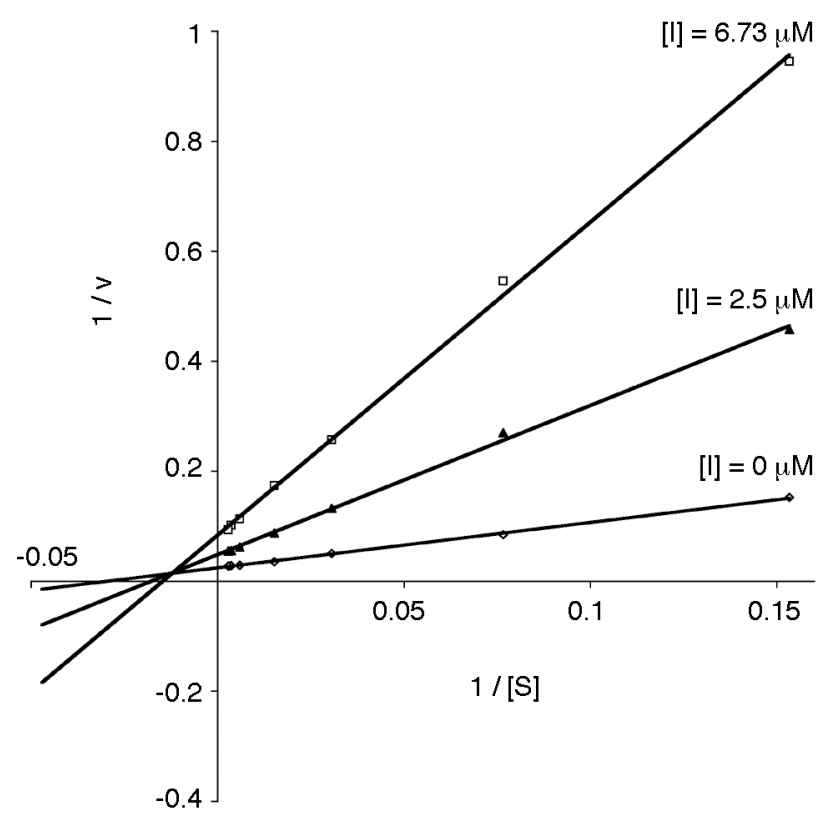

Figure IV-12. Lineweaver-Burk plot of AChE activity in the presence of IV-5.

\subsubsection{Fluorescence Spectroscopy.}

Solutions for fluorescence titrations were prepared in $20 \mathrm{mM} \mathrm{NaH} \mathrm{PO}_{4}$ Buffer, pH 7.3. All spectra were measured on a Hitachi F-4500 fluorescence spectrophotometer with excitation and emission band passes set at $5 \mathrm{~nm}$. The temperature was held constant at $25.0{ }^{\circ} \mathrm{C}$ using Neslab RTE-111 bath / circulator. An excitation wavelength of $290 \mathrm{~nm}$ was used to excite BCA. The change of fluorescence was measured by integrating the area (from 450 to $480 \mathrm{~nm}$ ) under each spectrum. 


\subsubsection{Measurement of the Binding Constant of the Inhibitor to BCA} using Fluorescence Spectroscopy.

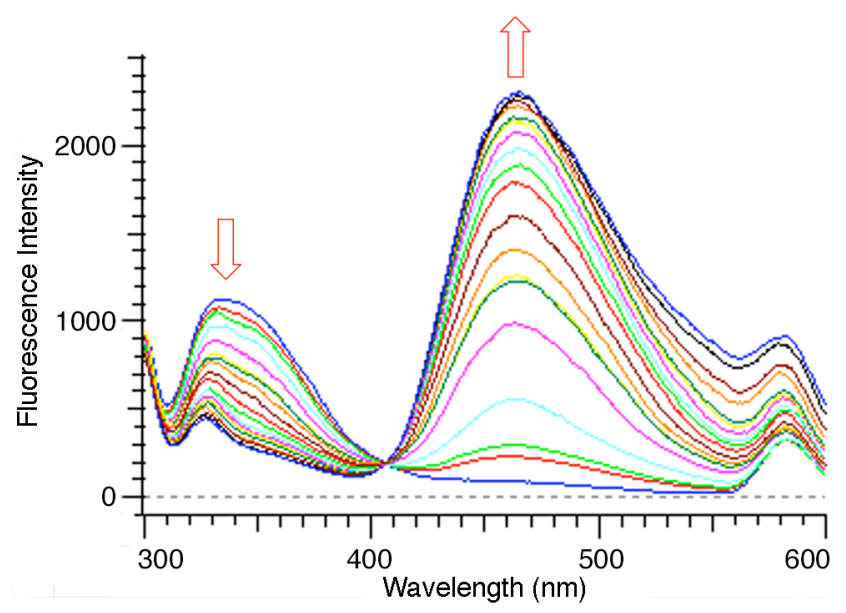

Figure IV-13. Plot of fluorescence intensity versus wavelength in the titration of a solution of BCA by IV-12 (20 mM NaH${ }_{2} \mathrm{PO}_{4}$ Buffer, $\left.\mathrm{pH} 7.3,298 \mathrm{~K}\right)$.
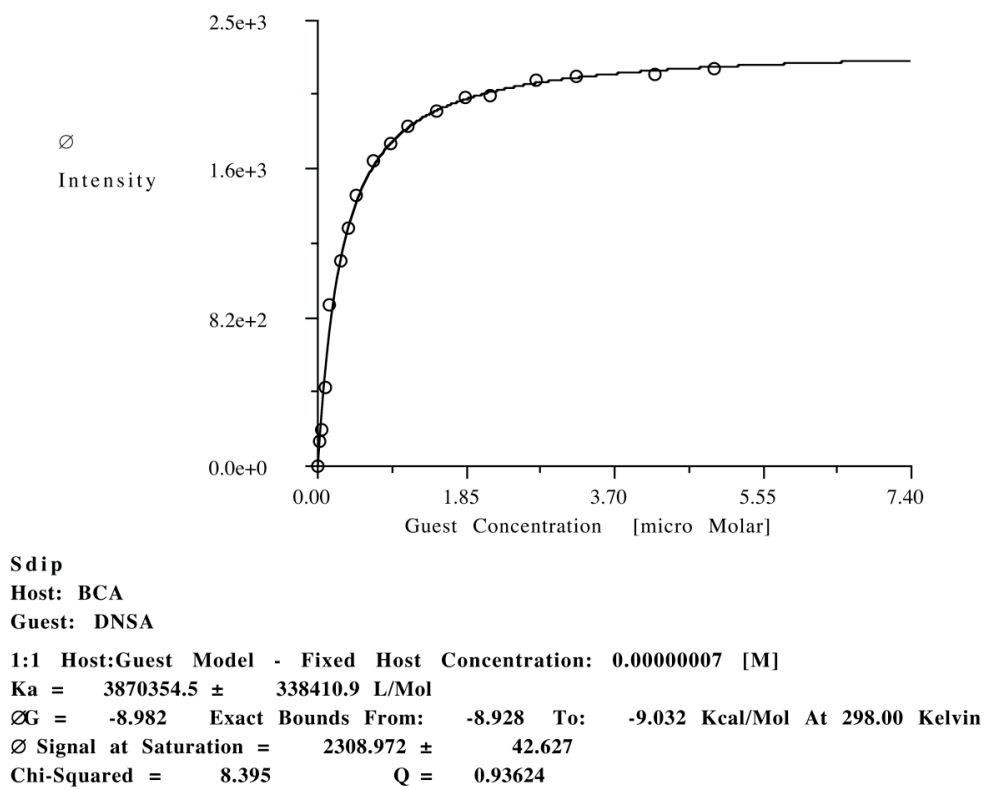

Figure IV-14. Determination of the $\mathrm{K}_{\mathrm{a}}$ value of $\mathbf{I V - 1 2}$ by nonlinear least-squares analysis fitting to a $1: 1$ binding model. 


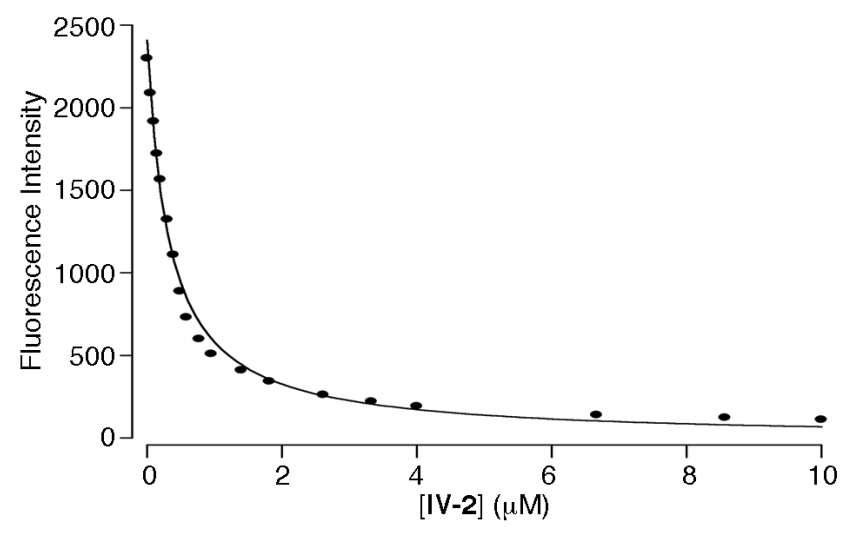

Figure IV-15. Plot of fluorescence intensity versus [IV-2] in the titration of BCA $・ I V-12$ with IV-2. Concentration of BCA (67.5 nM), IV-12 $(2 \mu \mathrm{M})$, and IV-2 $(50 \mathrm{nM}-10 \mu \mathrm{M})$. All measurements were done in $20 \mathrm{mM} \mathrm{NaH} \mathrm{PO}_{4}$ Buffer, $\mathrm{pH}$ 7.3, $298 \mathrm{~K}$. The value of $\mathrm{K}_{\mathrm{a}}$ is $(2.73 \pm 0.24) \times 10^{7} \mathrm{M}^{-1}$.

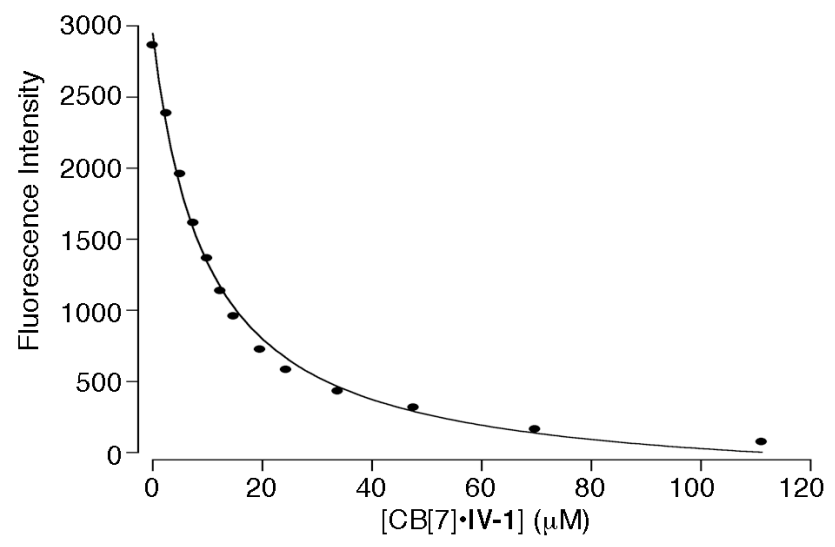

Figure IV-16. Plot of fluorescence intensity versus $[\mathrm{CB}[7] \bullet \mathbf{I V}-1]$ in the titration of BCA $\bullet I V-12$ with CB[7] $\bullet \mathbf{I V - 1 . ~ C o n c e n t r a t i o n ~ o f ~ B C A ~}(86 \mathrm{nM}), \mathbf{I V}-12(2 \mu \mathrm{M})$, and CB[7]•IV-1 $(2.5 \mathrm{nM}-120 \mu \mathrm{M})$. All measurements were done in $20 \mathrm{mM} \mathrm{NaH} \mathrm{PO}_{4}$ Buffer, pH 7.3, $298 \mathrm{~K}$. The value of $\mathrm{K}_{\mathrm{a}}$ is $(9.14 \pm 0.77) \times 10^{5} \mathrm{M}^{-1}$. 


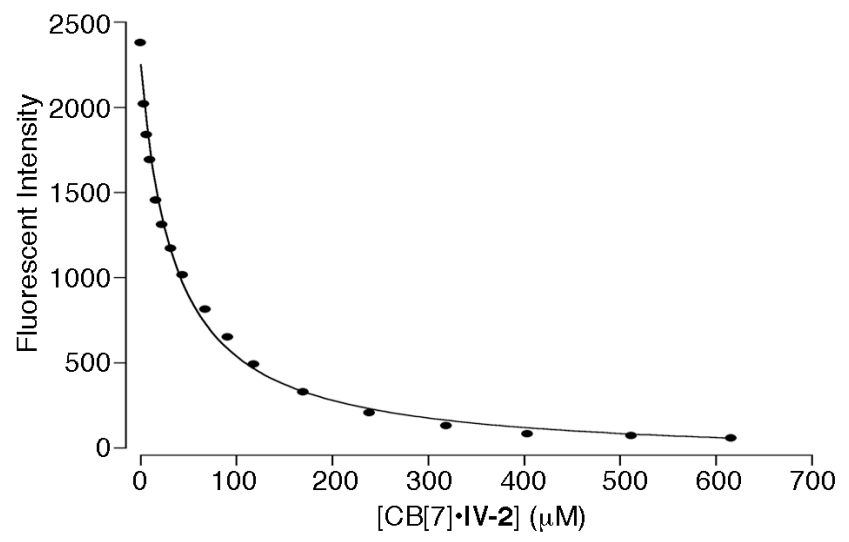

Figure IV-17. Plot of fluorescence intensity versus $[\mathrm{CB}[7] \cdot \mathbf{I V - 2}]$ in the titration of BCA $・ I V-12$ with CB[7] $\mathbf{I V - 2}$. Concentration of BCA $(86 \mathrm{nM}), \mathbf{I V}-\mathbf{1 2}(2 \mu \mathrm{M})$, and $\mathrm{CB}[7] \cdot \mathbf{I V}-2(6.5 \mathrm{nM}-400 \mu \mathrm{M})$. All measurements were done in $20 \mathrm{mM} \mathrm{NaH} \mathrm{PO}_{4}$ Buffer, $\mathrm{pH} 7.3,298 \mathrm{~K}$. The value of $\mathrm{K}_{\mathrm{a}}$ is $(2.56 \pm 0.21) \times 10^{5} \mathrm{M}^{-1}$. 


\subsubsection{Measurement of the Dissociation Constant of Different}

Sulfonamides from BCA:

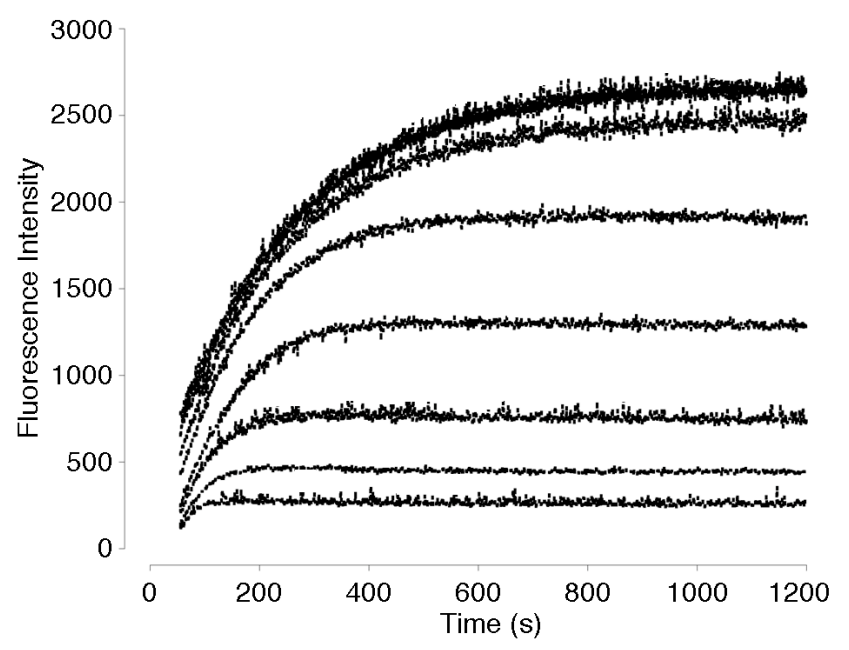

Figure IV-18. Plot of fluorescence intensity at $460 \mathrm{~nm}$ versus time after addition of IV-12 $(1.5 \mu \mathrm{M}-98 \mu \mathrm{M})$ to a solution containing BCA (80 nM) and IV-1 (2 $\mu \mathrm{M})(20$ $\mathrm{mM} \mathrm{NaH}{ }_{2} \mathrm{PO}_{4}$ Buffer, $\mathrm{pH}$ 7.3, $\left.298 \mathrm{~K}\right)$.

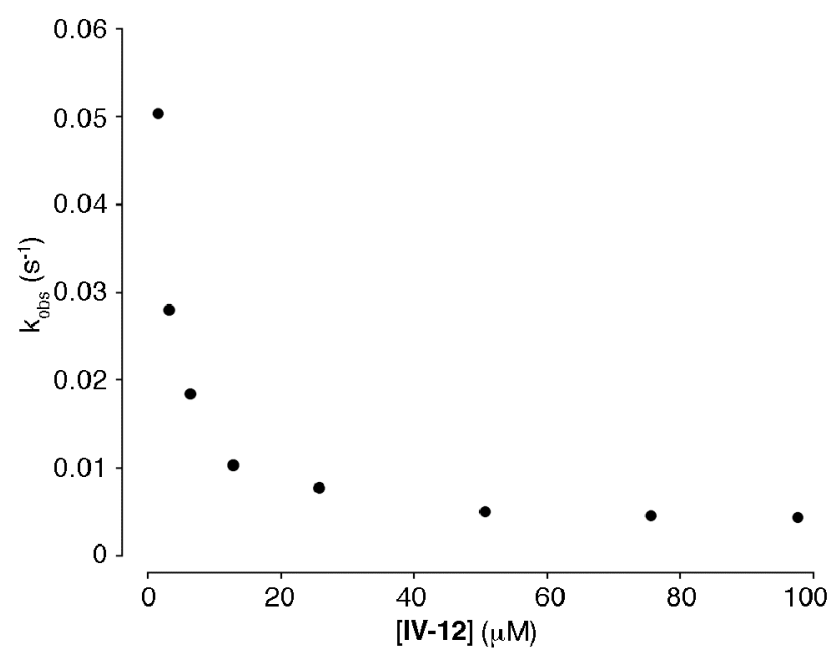

Figure IV-19. Plot of $\mathrm{k}_{\mathrm{obs}}$ for the dissociation of IV-1 from BCA versus concentration of IV-12. (20 mM NaH${ }_{2} \mathrm{PO}_{4}$ Buffer, pH 7.3, $298 \mathrm{~K}$ ). 


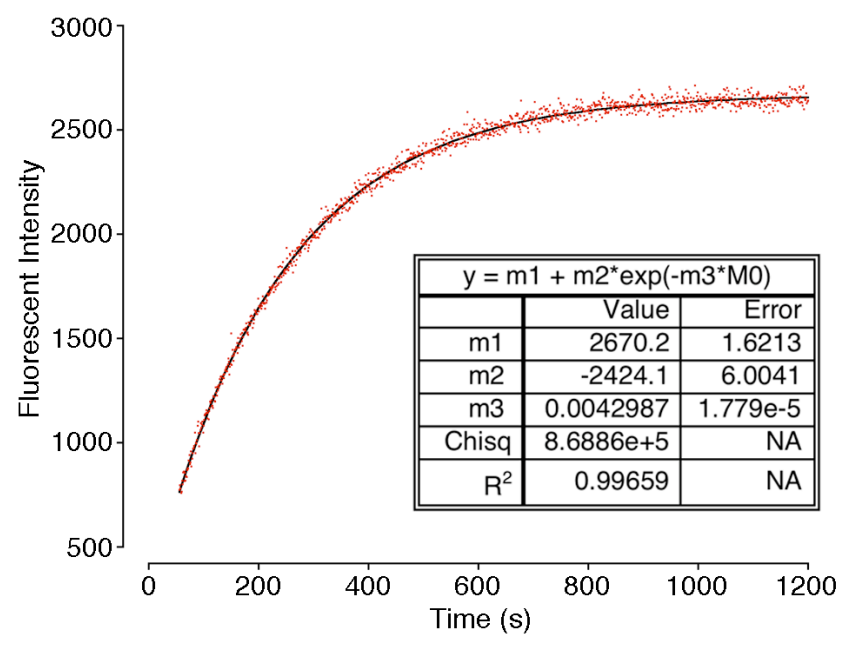

Figure IV-20. Plot of fluorescence intensity at $460 \mathrm{~nm}$ versus time after addition of IV-12 $(98 \mu \mathrm{M})$ to a solution containing BCA $(80 \mathrm{nM})$ and IV-1 $(2 \mu \mathrm{M})(20 \mathrm{mM}$ $\mathrm{NaH}_{2} \mathrm{PO}_{4}$ Buffer, pH 7.3, $298 \mathrm{~K}$ ).

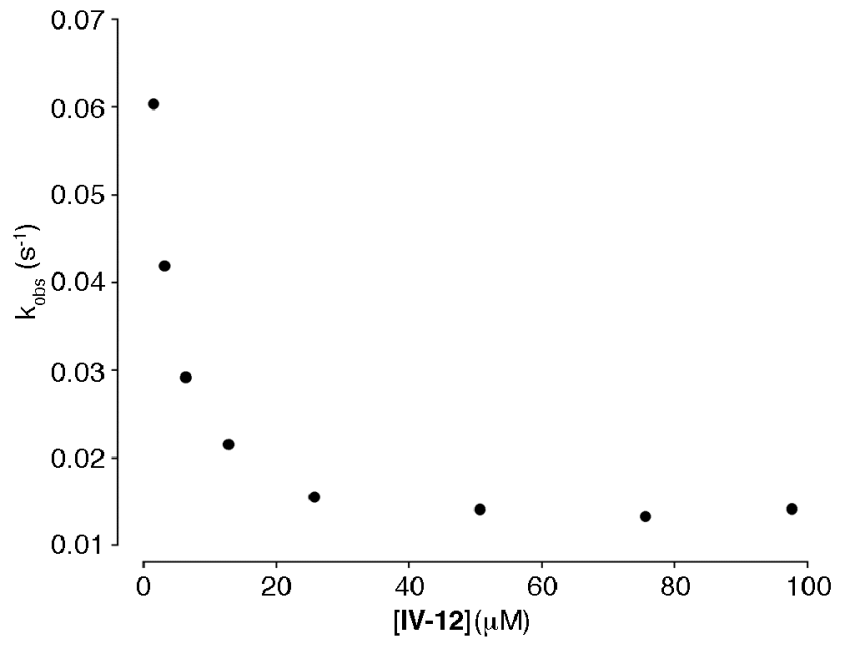

Figure IV-21. Plot of $\mathrm{k}_{\mathrm{obs}}$ for the dissociation of IV-2 from BCA versus concentration of IV-12. (20 mM NaH${ }_{2} \mathrm{PO}_{4}$ Buffer, $\mathrm{pH} 7.3,298 \mathrm{~K}$ ). 


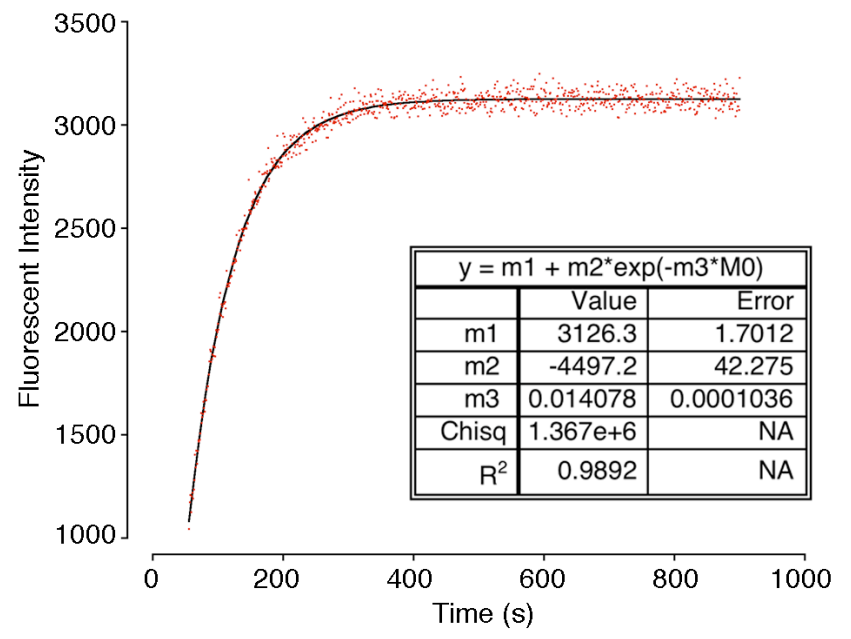

Figure IV-22. Plot of fluorescence intensity at $460 \mathrm{~nm}$ versus time after addition of IV-12 $(98 \mu \mathrm{M})$ to a solution containing BCA $(80 \mathrm{nM})$ and IV-2 $(2 \mu \mathrm{M})(20 \mathrm{mM}$ $\mathrm{NaH}_{2} \mathrm{PO}_{4}$ Buffer, pH 7.3, $\left.298 \mathrm{~K}\right)$.

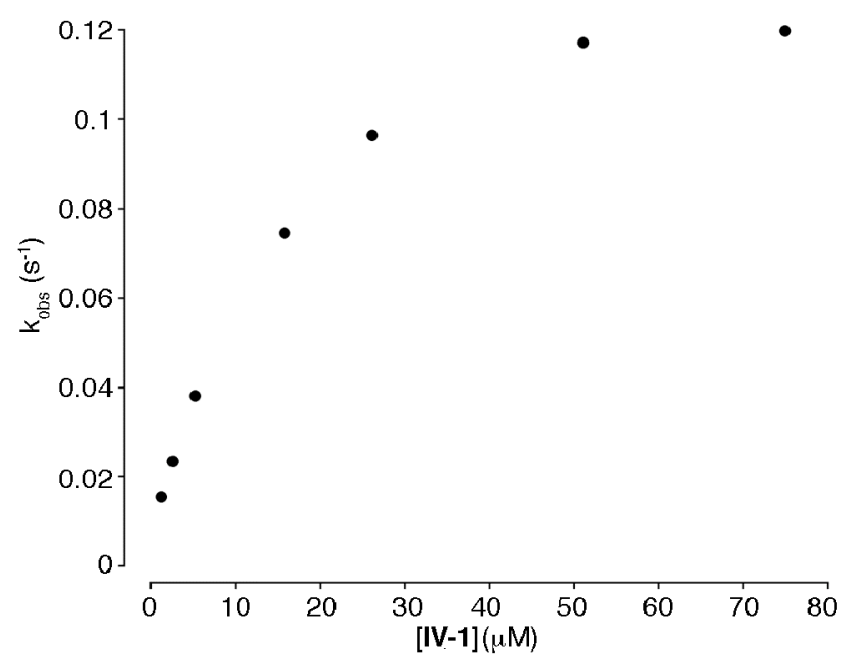

Figure IV-23. Plot of $\mathrm{k}_{\mathrm{obs}}$ for the dissociation of IV-12 from BCA versus concentration of 1. (20 mM NaH${ }_{2} \mathrm{PO}_{4}$ Buffer, $\left.\mathrm{pH} 7.3,298 \mathrm{~K}\right)$. 


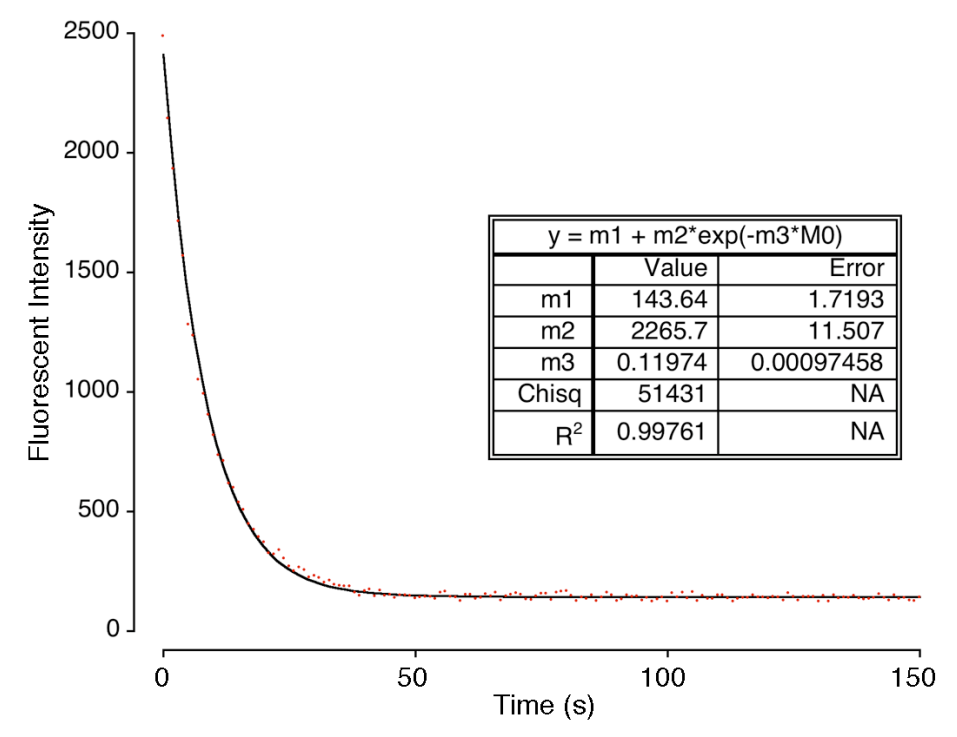

Figure IV-24. Plot of fluorescence intensity at $460 \mathrm{~nm}$ versus time after addition of IV-1 $(75 \mu \mathrm{M})$ to a solution containing BCA $(80 \mathrm{nM})$ and IV-12 $(2 \mu \mathrm{M})(20 \mathrm{mM}$ $\mathrm{NaH}_{2} \mathrm{PO}_{4}$ Buffer, pH 7.3, $298 \mathrm{~K}$ ).

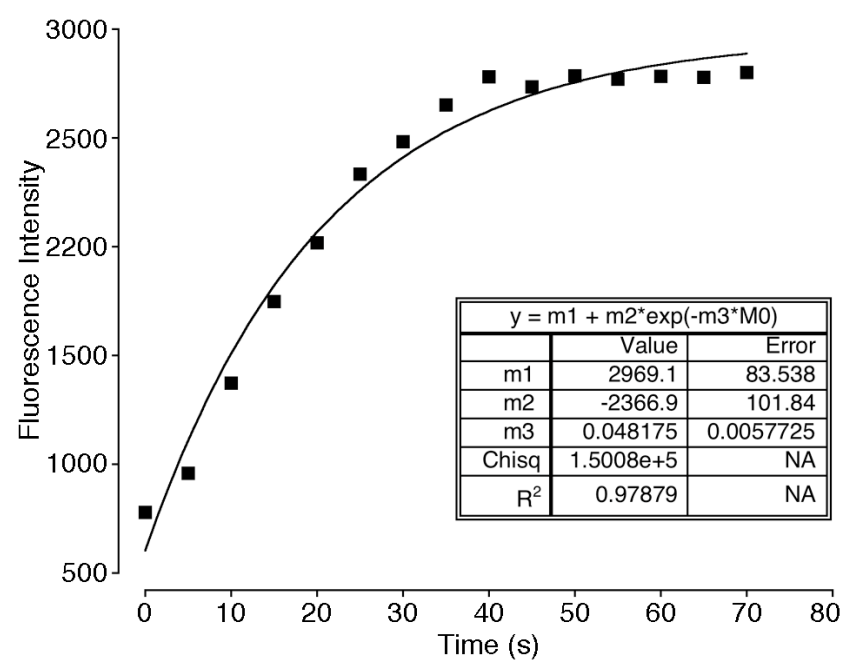

Figure IV-25. a) Plot of fluorescence intensity versus time after addition of $\mathrm{CB}$ [7] to a mixture of IV-12, IV-1 and BCA. Final concentrations of BCA, IV-12, IV-1 and $\mathrm{CB}$ [7] are $75 \mathrm{nM}, 25 \mu \mathrm{M}, 18 \mu \mathrm{M}$, and $50 \mu \mathrm{M}$ respectively; All measurements were done in $20 \mathrm{mM} \mathrm{NaH}{ }_{2} \mathrm{PO}_{4}$ Buffer, $\mathrm{pH} 7.3,298 \mathrm{~K}$. 


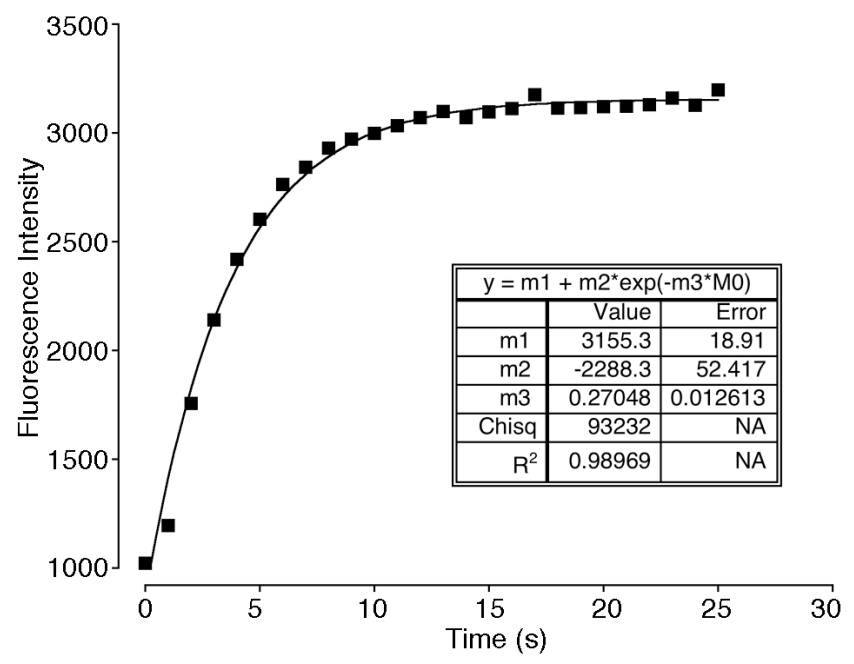

Figure IV-26. a) Plot of fluorescence intensity versus time after addition of $\mathrm{CB}$ [7] to a mixture of IV-12, IV-2 and BCA. Final concentrations of BCA, IV-12, IV-2 and $\mathrm{CB}$ [7] are $73 \mathrm{nM}, 25 \mu \mathrm{M}, 18 \mu \mathrm{M}$, and $50 \mu \mathrm{M}$ respectively; All measurements were done in $20 \mathrm{mM} \mathrm{NaH}{ }_{2} \mathrm{PO}_{4}$ Buffer, pH 7.3, $298 \mathrm{~K}$.

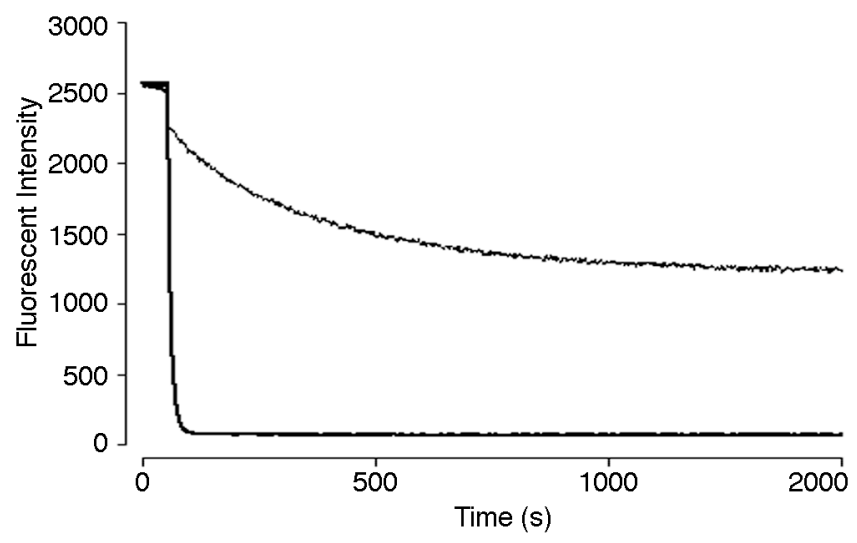

Figure IV-27. A comparison of decrease in fluorescence intensity of a solution containing IV-12 $(2 \mu \mathrm{M})$ and BCA $(80 \mathrm{nM})$ in presence of IV-1 $(20 \mu \mathrm{M})$ and CB[7]• IV-1 $(20 \mu \mathrm{M})$. All measurements were done in $20 \mathrm{mM} \mathrm{NaH} \mathrm{PO}_{4}$ Buffer, pH 7.3, 298 K. 

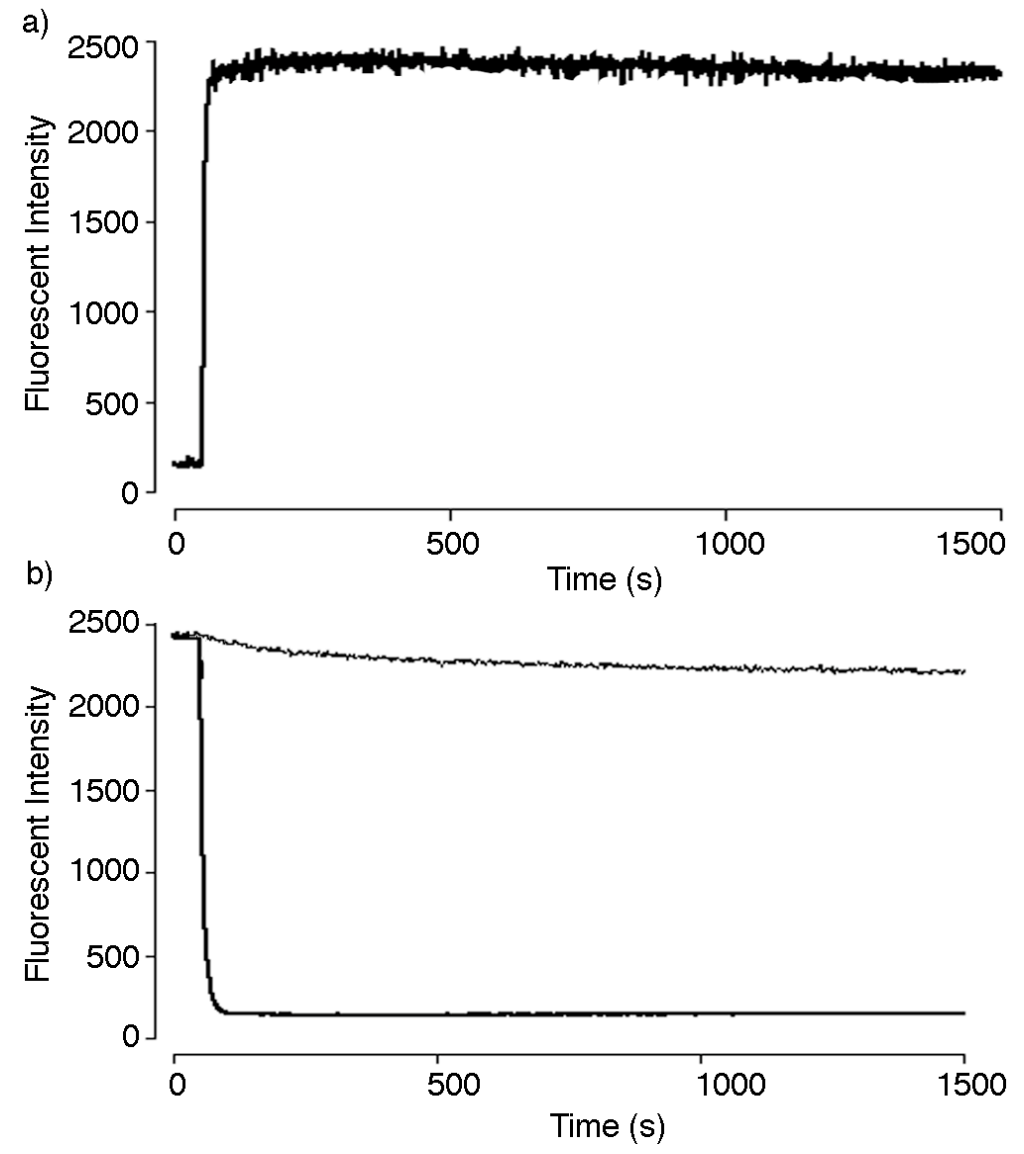

Figure IV-28. a) Plot of fluorescence intensity versus time after addition of $\mathrm{CB}[7]$ to a mixture of IV-12, IV-2 and BCA. Final concentrations of BCA, IV-12, IV-2 and $\mathrm{CB}[7]$ are $80 \mathrm{nM}, 2 \mu \mathrm{M}, 20 \mu \mathrm{M}$, and $50 \mu \mathrm{M}$ respectively; and b) A comparison of decrease in fluorescence intensity of a solution containing IV-12 $(2 \mu \mathrm{M})$ and BCA $(80 \mathrm{nM})$ in presence of IV-2 $(20 \mu \mathrm{M})$ and CB[7]-IV-2 $(20 \mu \mathrm{M})$. All measurements were done in $20 \mathrm{mM} \mathrm{NaH} \mathrm{PO}_{4}$ Buffer, pH 7.3, $298 \mathrm{~K}$. 


\section{Chapter 5: Summary and Future Work.}

\subsection{Summary.}

Analysis of complex systems is becoming an important area of research in chemistry to understand various fundamental problems concerning life such as how does a collection of molecules give rise to a specific behavior inside cell and organism. In this work, we demonstrated our approach to create functional and emergent behavior in a complex mixture using self-sorting. In chapter 2, we discussed how to control self- versus non-self- assembly of molecular clips in organic solution. In the contrary, Nature's self-sorting systems perform in aqueous solution. In chapter 3, we created a complex network by using host-guest self-assembly in water. We demonstrated several interesting features involved in that process using NMR spectroscopy and deconvoluted the large interaction network by using computational methods. In chapter 4, we demonstrated a use of the stimuli responsive nature of host-guest self-assembly process to control biological catalysis.

\section{$5.2 \quad$ Future Work.}

The research described in this work has broad applicability. Molecular clips have ester functional groups attached to the convex face of the cleft. These molecules can the attached to polymeric backbones to create new class of self-sorting polymers (Scheme V-1). There are various parameters that can affect the self-sorting process: 
rigidity of the polymeric backbone, number of molecular clips per oligomeric strand, sequence of molecular clips attached to single strand.

Scheme V-1. Self-sorting of polymer bound molecular clips.

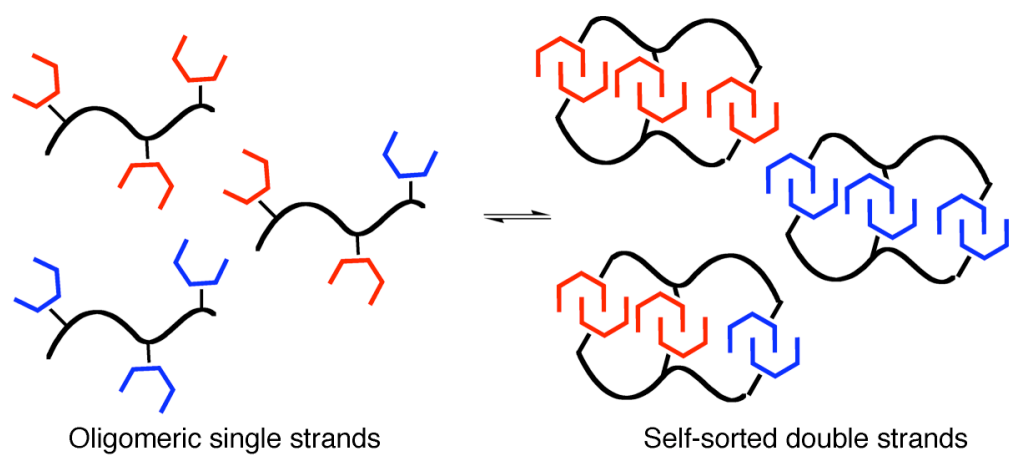

The complex interaction network described in chapter 3 is purely operational under thermodynamic control. To mimic the networks in biology more closely in future we are planning to incorporate biological receptors (e.g. proteins, enzymes), kinetically controlled steps, feedback loops, compartmentation in our design. The environmental responsiveness of host-guest self-assembly can be used to control the outcome of a multi-component mixture. For example, the activity of a particular enzyme in a mixture of multiple enzymes can be regulated by using the high binding affinity and selectivity of $\mathrm{CB}[\mathrm{n}]$ molecules (Scheme V-2). 
Scheme V-2. Selective regulation of the activity of an enzyme in a mixture.

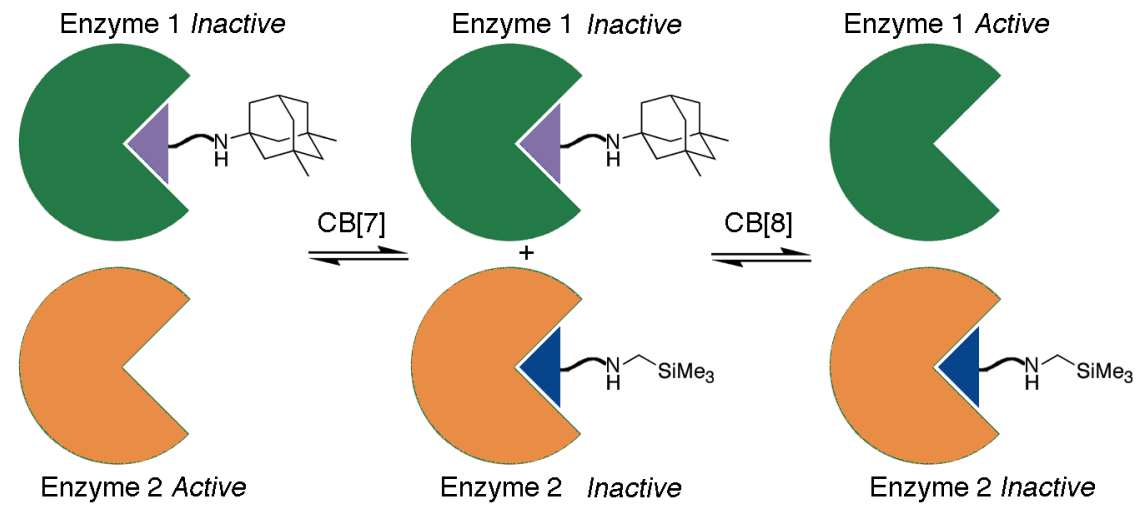

Finally, a biological receptor can be used in place of cucurbit[n]uril in scheme IV-1 where the $\mathrm{CB}[\mathrm{n}]$ binding epitope is replaced by biologically relevant guest molecule (e.g. inhibitor, biotin). The resulting two-faced guest can still regulate the activity of the receptors under thermodynamic control. The resulting system would be more compatible to biology and potentially promising for further in vivo applications. 


\section{Bibliography}

(1) Ghosh, S.; Isaacs, L. Complex Self-Sorting Systems. In Dynamic Combinatorial Chemistry in Drug Discovery, Bioorganic Chemistry, and Materials Science, Miller, B. L., Ed.;Wiley-VCH, in press.

(2) Cram, D. J. Angew. Chem., Int. Ed. Engl. 1988, 27, 1009-1020.

(3) Lehn, J. M. Angew. Chem., Int. Ed. Engl. 1988, 27, 89-112.

(4) Pedersen, C. J. Angew. Chem., Int. Ed. Engl. 1988, 27, 1021-1027.

(5) Oshovsky, G. V.; Reinhoudt, D. N.; Verboom, W. Angew. Chem., Int. Ed. 2007, 46, 2366-2393.

(6) Schenning, A. P. H. J.; Meijer, E. W. Chem. Commun. 2005, 3245-3258.

(7) Rebek, J., Jr. Angew. Chem., Int. Ed. 2005, 44, 2068-2078.

(8) Fujita, M.; Umemoto, K.; Yoshizawa, M.; Fujita, N.; Kusukawa, T.; Biradha, K. Chem. Commun. 2001, 509-518.

(9) Seidel, S. R.; Stang, P. J. Acc. Chem. Res. 2002, 35, 972-983.

(10) Wright, A. T.; Anslyn, E. V. Chem. Soc. Rev. 2006, 35, 14-28.

(11) McNally, B. A.; Leevy, W. M.; Smith, B. D. Supramol. Chem. 2007, 19, 2937. 
(12) Davis, A. P.; Sheppard, D. N.; Smith, B. D. Chem. Soc. Rev. 2007, 36, 348357.

(13) Kay, E. R.; Leigh, D. A.; Zerbetto, F. Angew. Chem., Int. Ed. 2007, 46, $72-$ 191.

(14) Kramer, R.; Lehn, J. M.; Marquis-Rigault, A. Proc. Natl. Acad. Sci. U. S. A. 1993, 90, 5394-5398.

(15) Rowan, S. J.; Hamilton, D. G.; Brady, P. A.; Sanders, J. K. M. J. Am. Chem. Soc. 1997, 119, 2578-2579.

(16) Mukhopadhyay, P.; Wu, A.; Isaacs, L. J. Org. Chem. 2004, 69, 6157-6164.

(17) Wu, A.; Chakraborty, A.; Witt, D.; Lagona, J.; Damkaci, F.; Ofori, M. A.;

Chiles, J. K.; Fettinger, J. C.; Isaacs, L. J. Org. Chem. 2002, 67, 5817-5830.

(18) Chakraborty, A.; Wu, A.; Witt, D.; Lagona, J.; Fettinger, J. C.; Isaacs, L. J. Am. Chem. Soc. 2002, 124, 8297-8306.

(19) Witt, D.; Lagona, J.; Damkaci, F.; Fettinger, J. C.; Isaacs, L. Org. Lett. 2000, 2, 755-758.

(20) Isaacs, L.; Witt, D. Angew. Chem., Int. Ed. 2002, 41, 1905-1907.

(21) Wu, A.; Chakraborty, A.; Fettinger, J. C.; Flowers, R. A., II; Isaacs, L. Angew. Chem., Int. Ed. 2002, 41, 4028-4031. 
(22) Cai, M.; Shi, X.; Sidorov, V.; Fabris, D.; Lam, Y.-F.; Davis, J. T. Tetrahedron 2002, 58, 661-671.

(23) Shi, X.; Fettinger, J. C.; Cai, M.; Davis, J. T. Angew. Chem., Int. Ed. 2000, 39, 3124-3127.

(24) Shi, X.; Fettinger, J. C.; Davis, J. T. J. Am. Chem. Soc. 2001, 123, 6738-6739.

(25) Castellano, R. K.; Nuckolls, C.; Rebek, J., Jr. J. Am. Chem. Soc. 1999, 121, $11156-11163$.

(26) Jolliffe, K. A.; Timmerman, P.; Reinhoudt, D. N. Angew. Chem., Int. Ed. 1999, 38, 933-937.

(27) Wyler, R.; de Mendoza, J.; Rebek, J., Jr. Angew. Chem., Int. Ed. Engl. 1993, $32,1699-1701$.

(28) Beijer, F. H.; Sijbesma, R. P.; Kooijman, H.; Spek, A. L.; Meijer, E. W. J. Am. Chem. Soc. 1998, 120, 6761-6769.

(29) Wu, A.; Isaacs, L. J. Am. Chem. Soc. 2003, 125, 4831-4835.

(30) Taylor, P. N.; Anderson, H. L. J. Am. Chem. Soc. 1999, 121, 11538-11545.

(31) Liu, S.; Ruspic, C.; Mukhopadhyay, P.; Chakrabarti, S.; Zavalij, P. Y.; Isaacs, L. J. Am. Chem. Soc. 2005, 127, 15959-15967.

(32) Mock, W. L.; Shih, N. Y. J. Org. Chem. 1986, 51, 4440-4446.

(33) Mock, W. L.; Shih, N. Y. J. Am. Chem. Soc. 1989, 111, 2697-2699. 
(34) Marquez, C.; Hudgins, R. R.; Nau, W. M. J. Am. Chem. Soc. 2004, 126, 58065816.

(35) Marquez, C.; Nau, W. M. Angew. Chem., Int. Ed. 2001, 40, 3155-3160.

(36) Mukhopadhyay, P.; Zavalij, P. Y.; Isaacs, L. J. Am. Chem. Soc. 2006, 128, 14093-14102.

(37) Liu, S.; Zavalij, P. Y.; Lam, Y.-F.; Isaacs, L. J. Am. Chem. Soc. 2007, 129, $11232-11241$.

(38) Maeda, C.; Kamada, T.; Aratani, N.; Osuka, A. Coord. Chem. Rev. 2007, 251, $2743-2752$

(39) Kamada, T.; Aratani, N.; Ikeda, T.; Shibata, N.; Higuchi, Y.; Wakamiya, A.; Yamaguchi, S.; Kim, K. S.; Yoon, Z. S.; Kim, D.; Osuka, A. J. Am. Chem. Soc. 2006, $128,7670-7678$.

(40) Hwang, I.-W.; Kamada, T.; Ahn, T. K.; Ko, D. M.; Nakamura, T.; Tsuda, A.; Osuka, A.; Kim, D. J. Am. Chem. Soc. 2004, 126, 16187-16198.

(41) Ma, Y.; Kolotuchin, S. V.; Zimmerman, S. C. J. Am. Chem. Soc. 2002, 124, 13757-13769.

(42) Corbin, P. S.; Lawless, L. J.; Li, Z.; Ma, Y.; Witmer, M. J.; Zimmerman, S. C. Proc. Natl. Acad. Sci. U. S. A. 2002, 99, 5099-5104.

(43) Lupas, A. Trends Biochem. Sci. 1996, 21, 375-382. 
(44) Bilgicer, B.; Kumar, K. Tetrahedron 2002, 58, 4105-4112.

(45) Bilgicer, B.; Xing, X.; Kumar, K. J. Am. Chem. Soc. 2001, 123, 11815-11816.

(46) Schnarr, N. A.; Kennan, A. J. J. Am. Chem. Soc. 2003, 125, 667-671.

(47) Schnarr, N. A.; Kennan, A. J. J. Am. Chem. Soc. 2002, 124, 9779-9783.

(48) Diss, M. L.; Kennan, A. J. J. Am. Chem. Soc. 2008, 130, 1321-1327.

(49) Schnarr, N. A.; Kennan, A. J. J. Am. Chem. Soc. 2003, 125, 13046-13051.

(50) Schnarr, N. A.; Kennan, A. J. J. Am. Chem. Soc. 2003, 125, 6364-6365.

(51) Sarma, R. J.; Nitschke, J. R. Angew. Chem., Int. Ed. 2008, 47, 377-380.

(52) Nitschke, J. R. Acc. Chem. Res. 2007, 40, 103-112.

(53) Xu, H.; Hong, R.; Lu, T.; Uzun, O.; Rotello, V. M. J. Am. Chem. Soc. 2006, $128,3162-3163$.

(54) Seeman, N. C. Mol. Biotechnol. 2007, 37, 246-257.

(55) He, Y.; Tian, Y.; Chen, Y.; Ribbe, A. E.; Mao, C. Chem. Commun. 2007, 165167.

(56) Rozenman, M. M.; McNaughton, B. R.; Liu, D. R. Curr. Opin. Chem. Biol. 2007, 11, 259-268.

(57) Kanan, M. W.; Rozenman, M. M.; Sakurai, K.; Snyder, T. M.; Liu, D. R. Nature 2004, 431, 545-549. 
(58) Gartner, Z. J.; Tse, B. N.; Grubina, R.; Doyon, J. B.; Snyder, T. M.; Liu, D. R. Science 2004, 305, 1601-1605.

(59) Calderone, C. T.; Puckett, J. W.; Gartner, Z. J.; Liu, D. R. Angew. Chem., Int. Ed. 2002, 41, 4104-4108.

(60) Burd, C.; Weck, M. Macromolecules 2005, 38, 7225-7230.

(61) Hirst, A. R.; Huang, B.; Castelletto, V.; Hamley, I. W.; Smith, D. K. Chem.-Eur. J. 2007, 13, 2180-2188.

(62) Ghosh, S.; Wu, A.; Fettinger, J. C.; Zavalij, P. Y.; Isaacs, L. J. Org. Chem. 2008, 73, 5915-5925.

(63) Corbett, P. T.; Leclaire, J.; Vial, L.; West, K. R.; Wietor, J.-L.; Sanders, J. K. M.; Otto, S. Chem. Rev. 2006, 106, 3652-3711.

(64) Lehn, J.-M. Chem. Eur. J. 1999, 5, 2455-2463.

(65) Jon, S. Y.; Ko, Y. H.; Park, S. H.; Kim, H.-J.; Kim, K. Chem. Commun. 2001, 1938-1939.

(66) Sisson, A. L.; Shah, M. R.; Bhosale, S.; Matile, S. Chem. Soc. Rev. 2006, 35, 1269-1286.

(67) Haag, R.; Kratz, F. Angew. Chem., Int. Ed. 2006, 45, 1198-1215.

(68) Yoshizawa, M.; Tamura, M.; Fujita, M. Science 2006, 312, 251-254.

(69) Pluth, M. D.; Bergman, R. G.; Raymond, K. N. Science 2007, 316, 85-88. 
(70) Pollino, J. M.; Weck, M. Chem. Soc. Rev. 2005, 34, 193-207.

(71) Pollino, J. M.; Stubbs, L. P.; Weck, M. J. Am. Chem. Soc. 2004, 126, 563-567.

(72) Balzani, V.; Credi, A.; Raymo, F. M.; Stoddart, J. F. Angew. Chem., Int. Ed. 2000, 39, 3348-3391.

(73) Sijbesma, R. P.; Meijer, E. W. Chem. Commun. 2003, 5-16.

(74) Ludlow, R. F.; Otto, S. Chem. Soc. Rev. 2008, 37, 101-108.

(75) Corbett, P. T.; Sanders, J. K. M.; Otto, S. Angew. Chem., Int. Ed. 2007, 46, $8858-8861$

(76) Kindermann, M.; Stahl, I.; Reimold, M.; Pankau Wolf, M.; von Kiedrowski, G. Angew. Chem., Int. Ed. 2005, 44, 6750-5.

(77) Wintner, E. A.; Conn, M. M.; Rebek, J. J. Acc. Chem. Res. 1994, 27, 198-203.

(78) Bachmann, P. A.; Luisi, P. L.; Lang, J. Nature 1992, 357, 57-9.

(79) Terfort, A.; Von Kiedrowski, G. Angew. Chem., Int. Ed. Engl. 1992, 31, 654656.

(80) Lee, D. H.; Severin, K.; Yokobayashi, Y.; Ghadiri, M. R. Nature 1997, 390, $591-594$.

(81) Sievers, D.; von Kiedrowski, G. Chem. Eur. J. 1998, 4, 629-641.

(82) Yao, S.; Ghosh, I.; Zutshi, R.; Chmielewski, J. Nature 1998, 396, 447-450. 
(83) Kassianidis, E.; Philp, D. Angew. Chem., Int. Ed. 2006, 45, 6344-6348.

(84) Chakrabarti, S.; Mukhopadhyay, P.; Lin, S.; Isaacs, L. Org. Lett. 2007, 9, 2349-2352.

(85) Rowan, A. E.; Elemans, J. A. A. W.; Nolte, R. J. M. Acc. Chem. Res. 1999, 32, 995-1006.

(86) Zimmerman, S. C. Top. Curr. Chem. 1993, 165, 71-102.

(87) Klaerner, F.-G.; Kahlert, B. Acc. Chem. Res. 2003, 36, 919-932.

(88) Harmata, M. Acc. Chem. Res. 2004, 37, 862-873.

(89) Jiang, H.; Leger, J.-M.; Guionneau, P.; Huc, I. Org. Lett. 2004, 6, 2985-2988.

(90) Chiang, P.-T.; Cheng, P.-N.; Lin, C.-F.; Liu, Y.-H.; Lai, C.-C.; Peng, S.-M.; Chiu, S.-H. Chem. Eur. J. 2006, 12, 865-876.

(91) Petitjean, A.; Khoury, R. G.; Kryritsakas, N.; Lehn, J.-M. J. Am. Chem. Soc. 2004, 126, 6637-6647.

(92) Artacho, J.; Nilsson, P.; Bergquist, K.-E.; Wendt, O. F.; Wärnmark, K. Chem. Eur. J. 2006, 12, 2692-2701.

(93) Wu, A.; Mukhopadhyay, P.; Chakraborty, A.; Fettinger, J. C.; Isaacs, L. J. Am. Chem. Soc. 2004, 126, 10035-10043.

(94) Barrett, E. S.; Dale, T. J.; Rebek, J., Jr. J. Am. Chem. Soc., 2008, 130, 23442350. 
(95) Braekers, D.; Peters, C.; Bogdan, A.; Rudzevich, Y.; Boehmer, V.; Desreux, J. F. J. Org. Chem. 2008, 73, 701-706.

(96) He, Y.; Tian, Y.; Chen, Y.; Ribbe, A. E.; Mao, C. Chem. Commun. 2007, 165167.

(97) Hutin, M.; Cramer, C. J.; Gagliardi, L.; Shahi, A. R. M.; Bernardinelli, G.; Cerny, R.; Nitschke, J. R. J. Am. Chem. Soc. 2007, 129, 8774-8780.

(98) Legrand, Y.-M.; Van der Lee, A.; Barboiu, M. Inorg. Chem. 2007, 46, 95409547.

(99) Plutowski, U.; Jester, S. S.; Lenhert, S.; Kappes, M. M.; Richert, C. Adv. Mater. 2007, 19, 1951-1956.

(100) Rowan, S. J.; Reynolds, D. J.; Sanders, J. K. M. J. Org. Chem. 1999, 64, 5804-5814.

(101) Saur, I.; Scopelliti, R.; Severin, K. Chem. Eur. J. 2006, 12, 1058-1066.

(102) Telfer, S. G.; Sato, T.; Kuroda, R.; Lefebvre, J.; Leznoff, D. B. Inorg. Chem. 2004, 43, 421-429.

(103) Uzun, O.; Xu, H.; Jeoung, E.; Thibault, R. J.; Rotello, V. M. Chem. Eur. J. 2005, 11, 6916-6920.

(104) Yoder, N. C.; Kumar, K. J. Am. Chem. Soc. 2006, 128, 188-191. 
(105) Wang, Z.-G.; Zhou, B.-H.; Chen, Y.-F.; Yin, G.-D.; Li, Y.-T.; Wu, A.-X.; Isaacs, L. J. Org. Chem. 2006, 71, 4502-4508.

(106) Gut, D.; Rudi, A.; Kopilov, J.; Goldberg, I.; Kol, M. J. Am. Chem. Soc. 2002, $124,5449-5456$.

(107) Marguly, E.; McDonald, R.; Branda, N. R. Org. Lett. 2000, 2, 3169-3172.

(108) Mann, K. G.; Butenas, S.; Brummel, K. Arterioscler., Thromb., Vasc. Biol. 2003, 23, 17-25.

(109) Milo, R.; Shen-Orr, S.; Itzkovitz, S.; Kashtan, N.; Chklovskii, D.; Alon, U. Science 2002, 298, 824-827.

(110) Hartwell, L. H.; Hopfield, J. J.; Leibler, S.; Murray, A. W. Nature 1999, 402, C47-C52.

(111) Bonneau, R. Nat. Chem. Biol. 2008, 4, 658-664.

(112) Maslov, S.; Sneppen, K. Science 2002, 296, 910-913.

(113) Costanzo, M. C.; Crawford, M. E.; Hirschman, J. E.; Kranz, J. E.; Olsen, P.; Robertson, L. S.; Skrzypek, M. S.; Braun, B. R.; Hopkins, K. L.; Kondu, P.; Lengieza, C.; Lew-Smith, J. E.; Tillberg, M.; Garrels, J. I. Nucl. Acids Res. 2001, 29, 75-79.

(114) Ito, T.; Chiba, T.; Ozawa, R.; Yoshida, M.; Hattori, M.; Sakaki, Y. Proc. Natl. Acad. Sci. U. S. A. 2001, 98, 4569-4574. 
(115) Faith, J. J.; Hayete, B.; Thaden, J. T.; Mogno, I.; Wierzbowski, J.; Cottarel, G.; Kasif, S.; Collins, J. J.; Gardner, T. S. PLoS Biol. 2007, 5, 54-66.

(116) Albeck John, G.; MacBeath, G.; White Forest, M.; Sorger Peter, K.; Lauffenburger Douglas, A.; Gaudet, S. Nat. Rev. Mol. Cell Biol. 2006, 7, 803-812.

(117) Janes Kevin, A.; Yaffe Michael, B. Nat. Rev. Mol. Cell Biol. 2006, 7, 820828.

(118) Komili, S.; Silver, P. A. Nat. Rev. Genet. 2008, 9, 38-48.

(119) Aloy, P.; Russell, R. B. Nat. Rev. Mol. Cell Biol. 2006, 7, 188-197.

(120) Kell, D. B.; Brown, M.; Davey, H. M.; Dunn, W. B.; Spasic, I.; Oliver, S. G. Nat. Rev. Microbiol. 2005, 3, 557-565.

(121) Westerhoff, H. V.; Palsson, B. O. Nat. Biotechnol. 2004, 22, 1249-1252.

(122) Stephanopoulos, G.; Alper, H.; Moxley, J. Nat. Biotechnol. 2004, 22, 12611267.

(123) Oltvai, Z. N.; Barabasi, A.-L. Science 2002, 298, 763-764.

(124) Kitano, H. Science 2002, 295, 1662-1664.

(125) Fitzgerald, J. B.; Schoeberl, B.; Nielsen, U. B.; Sorger, P. K. Nat. Chem. Biol. 2006, 2, 458-466.

(126) Butcher, E. C. Nat. Rev. Drug Discovery 2005, 4, 461-467. 
(127) Hood, L.; Heath, J. R.; Phelps, M. E.; Lin, B. Science 2004, 306, 640-643.

(128) Whitesides, G. M.; Ismagilov, R. F. Science 1999, 284, 89-92.

(129) Kastrup, C. J.; Runyon, M. K.; Lucchetta, E. M.; Price, J. M.; Ismagilov, R. F. Acc. Chem. Res 2008, 41, 549-558.

(130) Ashkenasy, G.; Ghadiri, M. R. J. Am. Chem. Soc. 2004, 126, 11140-1.

(131) Buryak, A.; Severin, K. J. Comb. Chem. 2006, 8, 540-543.

(132) Corbett, P. T.; Otto, S.; Sanders, J. K. M. Chem. Eur. J. 2004, 10, 3139-3143.

(133) Corbett, P. T.; Sanders, J. K. M.; Otto, S. J. Am. Chem. Soc. 2005, 127, 93909392.

(134) Corbett, P. T.; Sanders, J. K. M.; Otto, S. Angew. Chem., Int. Ed. 2007, 46, 8858-8861.

(135) Hayden, D. J.; von Kiedrowski, G.; Lehman, N. Angew. Chem., Int. Ed. 2008, $47,8424-8428$.

(136) Kindermann, M.; Stahl, I.; Reimold, M.; Pankau, W. M.; von Kiedrowski, G. Angew. Chem., Int. Ed 2005, 44, 6750-6755.

(137) Ludlow, R. F.; Otto, S. Chem. Soc. Rev. 2008, 37, 101-108.

(138) Lagona, J.; Mukhopadhyay, P.; Chakrabarti, S.; Isaacs, L. Angew. Chem., Int. Ed. 2005, 44, 4844-4870. 
(139) Lee, J. W.; Samal, S.; Selvapalam, N.; Kim, H.-J.; Kim, K. Acc. Chem. Res. 2003, 36, 621-630.

(140) Rekharsky, M. V.; Inoue, Y. Chem. Rev. 1998, 98, 1875-1917.

(141) In (molecular) musical chairs, $n$ children (guests) march to music around $n-1$ chairs (hosts); when the music stops $n$ children compete for $n-1$ chairs. One of the children (guests) does not find a chair (hosts). The construction of complex selfsorting mixture resembles the microscopic reverse of this system

(142) A six component mixture III-1, III-2, III-3, III-5, III-6, and III-8 forms a self-sorted state consist of III-1•III-6 $(\chi=0.99)$, III-2•III-5 $(\chi=0.99)$, and III-3•III$8(\chi=0.99)\left(\Delta \mathrm{G}=-28.9 \mathrm{kcal} \mathrm{mol}^{-1}\right)$. Addition of III-4 results in a non self-selfsorted seven component mixture containing III-1•III-6 $(\chi=0.99)$, III-2 $(\chi=0.47)$, III-3 $(\chi=0.43)$, III-2•III-5 $(\chi=0.52)$, III-3•III-5 $(\chi=0.47), \mathbf{I I I}-3 \cdot I I I-8(\chi=0.85)$ and III-4•III-8 $(\chi=0.91)\left(\Delta \mathrm{G}=-31.1 \mathrm{kcal} \mathrm{mol}^{-1}\right)$ and the transformation is associated with $\Delta \Delta \mathrm{G}=-2.2 \mathrm{kcal} \mathrm{mol}^{-1}$. Whereas, another six component mixture of III-1, III-2, III-4, III-5, III-6, and III-8 forms a self-sorted state consist of III-1•III$\mathbf{6}(\chi=0.99)$, III-2•III-5 $(\chi=0.99)$, and III-4•III-8 $(\chi=1.00)$ is associated with $\Delta \mathrm{G}=$ $-31.3 \mathrm{kcal} \mathrm{mol}^{-1}$. Addition III-3 results in same non self-self-sorted mixture containing III-1•III-6, III-2, III-3, III-2•III-5, III-3•III-5, and III-4•III-8 and the transformation is associated with $\Delta \Delta \mathrm{G}=0.2 \mathrm{kcal} \mathrm{mol}^{-1}$.

(143) Houk, K. N.; Leach, A. G.; Kim, S. P.; Zhang, X. Angew. Chem., Int. Ed. 2003, 42, 4872-4897. 
(144) Zhang, X.; Houk, K. N. Acc. Chem. Res. 2005, 38, 379-385.

(145) Stryer, L., Biochemistry 1995; Vol. 4th ed.

(146) Perutz, M. F.; Fermi, G.; Luisi, B.; Shaanan, B.; Liddington, R. C. Acc. Chem. Res. 1987, 20, 309-321.

(147) Rabinowitz, J. D.; Hsiao, J. J.; Gryncel, K. R.; Kantrowitz, E. R.; Feng, X.-J.; Li, G.; Rabitz, H. Biochemistry 2008, 47, 5881-5888.

(148) Wang, J.; Eldo, J.; Kantrowitz, E. R. J. Mol. Biol. 2007, 371, 1261-1273.

(149) Hammes, G. G. Biochemistry 2002, 41, 8221-8228.

(150) Hennig, A.; Bakirci, H.; Nau, W. M. Nat. Methods, 2007, 4, 629-632.

(151) Vomasta, D.; Hogner, C.; Branda Neil, R.; Konig, B. Angew. Chem., Int. Ed. 2008, $47,7644-7647$.

(152) Gianneschi, N. C.; Ghadiri, M. R. Angew. Chem., Int. Ed. 2007, 46, 39553958.

(153) Walsh, J. J.; Coughlan, D.; Heneghan, N.; Gaynor, C.; Bell, A. Bioorg. Med. Chem. Lett. 2007, 17, 3599-3602.

(154) Burgess, S. J.; Selzer, A.; Kelly, J. X.; Smilkstein, M. J.; Riscoe, M. K.; Peyton, D. H. J. Med. Chem. 2006, 49, 5623-5625.

(155) Hulsman, N.; Medema, J. P.; Bos, C.; Jongejan, A.; Leurs, R.; Smit, M. J.; de Esch, I. J. P.; Richel, D.; Wijtmans, M. J. Med. Chem. 2007, 50, 2424-2431. 
(156) Gajar, S. A.; Tano, T.; Resende, A. C.; Bitencourt, J. A. F.; de Lemos Neto, M.; Damiao, R.; Criddle, D. N.; Soares de Moura, R. BJU Int. 2007, 100, 1322-1325.

(157) Simon, A.; Barabas, P.; Kardos, J. Neurochem. Int. 2006, 49, 215-222.

(158) Marco, J. L.; Carreiras, M. C. Mini-Rev. Med. Chem. 2003, 3, 518-524.

(159) Ho, R. I.; Corman, L.; Morse, S. A.; Schneider, H. Antimicrob. Agents Chemother. 1975, 7, 758-763.

(160) Phenix, P.; Welbourne, T. C. Am. J. Physiol. 1975, 228, 1269-1275.

(161) Supuran, C. T.; Scozzafava, A. Bioorg. Med. Chem. 2007, 15, 4336-4350.

(162) Krishnamurthy, V. M.; Kaufman, G. K.; Urbach, A. R.; Gitlin, I.; Gudiksen, K. L.; Weibel, D. B.; Whitesides, G. M. Chem. Rev. 2008, 108, 946-1051.

(163) Whitesides George, M.; Krishnamurthy Vijay, M. Q. Rev. Biophys. 2005, 38, 385-395.

(164) Pocker, Y.; Stone, J. T. Biochemistry 1967, 6, 668-678.

(165) Rozema, D.; Gellman, S. H. J. Am. Chem. Soc. 1995, 117, 2373-2374.

(166) Rozema, D.; Gellman, S. H. J. Biol. Chem. 1996, 271, 3478-3487.

(167) Ellman, G. L.; Courtney, K. D.; Andres, V., Jr.; Featherstone, R. M. Biochem. Pharmacol. 1961, 7, 88-95. 
(168) The association constant of $\mathbf{I V - 8}$ to $\mathrm{CB}[7]\left(\mathrm{K}_{\mathrm{a}}=3.6 \times 10^{13} \mathrm{M}^{-1}\right)$ was measured by competition with adamantane amine by using ${ }^{1} \mathrm{H}$ NMR competition experiments for a limiting quantity of $\mathrm{CB}[7]$.

(169) The numbers obtained from these experiments were used to rationalize the experimental data assuming they would be the same under the experimental condition (30 mM NaD $2 \mathrm{PO}_{4}$ Buffer, $(\mathrm{pD} 7.3)$ at $25^{\circ} \mathrm{C}$.

(170) Binding constant of hexylamine and hexanediamine to $\mathrm{CB}[6]$ are around $10^{8}$ $\mathrm{M}^{-1}$ and $10^{7} \mathrm{M}^{-1}$ respectively. We observed approximately 10 -fold decrease in binding constant of hexanediamine when the size of host is increase from $\mathrm{CB}[6]$ to $\mathrm{CB}[7]\left(\sim 10^{7} \mathrm{M}^{-1}\right)$ to $\mathrm{CB}[8]\left(10^{6} \mathrm{M}^{-1}\right)$.

(171) Banerjee, A. L.; Tobwala, S.; Ganguly, B.; Mallik, S.; Srivastava, D. K. Biochemistry 2005, 44, 3673-3682.

(172) Krishnamurthy, V. M.; Semetey, V.; Bracher, P. J.; Shen, N.; Whitesides, G. M. J. Am. Chem. Soc. 2007, 129, 1312-1320.

(173) Nair, S.; Krebs, J. F.; Christianson, D. W.; Fierke, C. A. Biochemistry 1995, 34, 3981-3989.

(174) Porcelli, F.; Delfini, M.; Del Giudice, M. R. Bioorg. Chem. 1999, 27, 197205.

(175) Berman, H. A.; Leonard, K. Mol. Pharmacol. 1992, 41, 412-418.

(176) Decker, M. J. Med. Chem. 2006, 49, 5411-5413. 
(177) Koner, A. L.; Nau, W. M. Supramol. Chem. 2007, 19, 55-66.

(178) Kemp, S.; Wheate, N. J.; Stootman, F. H.; Aldrich-Wright, J. R. Supramol. Chem. 2007, 19, 475-484.

(179) Hennig, A.; Ghale, G.; Nau, W. M. Chem. Commun. 2007, 1614-1616.

(180) Saleh, N. i.; Koner, A. L.; Nau, W. M. Angew. Chem., Int. Ed. 2008, 47, 53985401.

(181) Lee, S. L.; Heinemann, S.; Taylor, P. J. Biol. Chem. 1982, 257, 12283-12291.

(182) Sussman, J. L.; Harel, M.; Frolow, F.; Oefner, C.; Goldman, A.; Toker, L.; Silman, I. Science 1991, 253, 872-879.

(183) Botti, S. A.; Felder, C. E.; Lifson, S.; Sussman, J. L.; Silman, I. Biophys. J. 1999, 77, 2430-2450.

(184) Bhasikuttan, A. C.; Mohanty, J.; Nau, W. M.; Pal, H. Angew. Chem., Int. Ed. 2007, 46, 4120-4122. 